\title{
INTEGRAL CHARGED PARTICLE NUCLEAR DATA BIBLIOGRAPHY
}

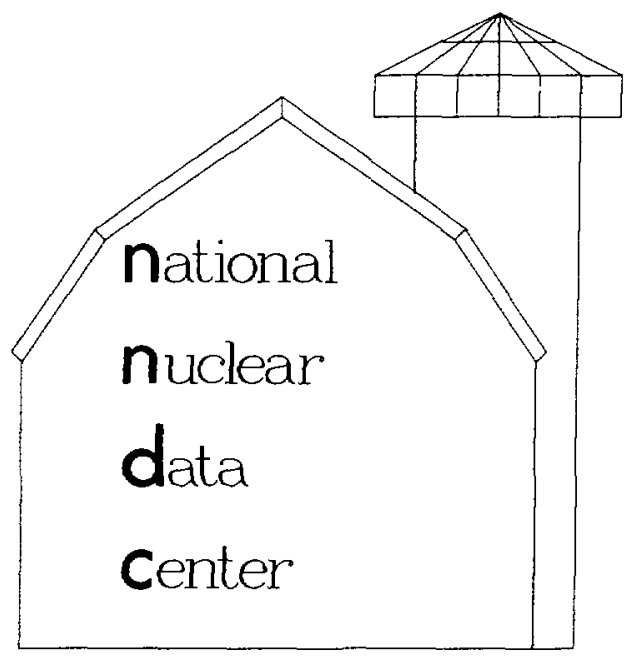

December 1988

(in support of the International Nuclear Reaction Data Centers Network) BROOEHAYEN NATIONAL LABORATORY A S S OCIATED UNIVERSITIES, INC. UNDER CONTRACT NO. DE-ACO2-76CHODDIG WITH THE UNITED STATES DEPARTMENT OF ENERGY 


\section{DISCLAIMER}

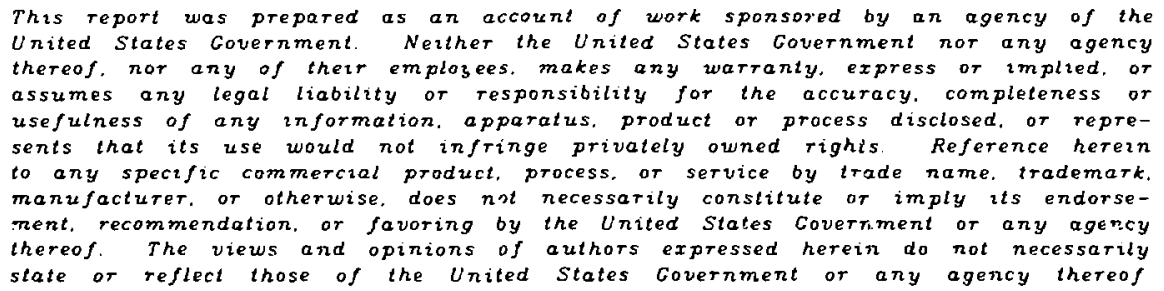

Printed in the United States of America

Avajlable from

National Technical Information Service

U.S. Department of Commerce

5285 Port Roya) Road

springfield, VA 2216i

NTIS Price Codes:

Printed Copy:A04;Microfiche:A01 


\section{INTRODUCTION}

I. Introduction

1I. Scope

III. Dates of Coverage

IV. Secondary Sources of Information

vi i

v. organization

VI. Compilations and Evaluations

$\mathrm{vii}$

VIl. Methods of Radioisotope Production

viii

VIII .

Retrieval Capabilities

viij

IX. Acknowledgements

REFERENCES

TARGET INDEX 


\section{INTRODUCTION}

This publication is the annual supplement to the first edition published in 1984 The primary goal of this publication has been to satisfy the need expressed by the Nuclear Reaction Data Center Network" for a concise and comprehensive bibliography of integral charged-particle cross section data. The reader is referred to Table 2 ror a partial list of other bibliographies relevant to charged-particle-induced reaction data and to "A Source List of Nuclear Data Bibliographies, Compilations, and Evaluations"l for a more comprehensive list. Since this publication is not cumulative, earlier versions are also shown in Table 2.

This publication makes use of a modification to the database of the Nuclear Structure References (NSR) file (see the first entry in Table 2). This modification allows the retrieval of integral charged particle nuclear data entries from the NSR file. In recent years, the presentation of various sections was changed, as a result of users' suggestions. The authors continue to welcome users' comments.

\section{Table 1}

\section{Centers Participating in the Compilation and Exchange of Charged-Particle Nuclear Data $\dagger$}

Center for Nuclear Structure and Reaction Data of the USSR State Committee on the Utilization of Atomic Energy, I.V.Kurchatov Institute of Atomic Energy,

Moscow, USSR

Service area. Union of Sovzet Socialist Republics

National Nuclear Data Center, Brookhaven National Laboratory

Upton, NY USA 11973

Service area. Unzted States and Canada

Nuclear Data Section. International Atomic Energy Agency

P.O.B. 100, A-1400,

Vienna, Austria

Service area: Non-OECD countries except for the USSR

Nuclear Energy Agency Data Bank

B.P. $9, F-91190$

Gif-Sur-Yvette, France

Service area OECD countries except for the United States and Canada

Nuclear Data Group

RIKEN Institute of Physical and Chemical Research

Wako-Shi, Saitama, Japan 351-01

Chinese Nuclear Data Center Institute of Atomic Energy, Acadamia Sinica, P.O.Box 275

Beijing, China

- See Table I and the introduction to CINDA, The Index to Literature and Computer Fales on Microscopac Neutron Data. published by the International Atomic Energy Agency, Vienna, for members of the network.

1 T.W. Burrows and N.E. Holden. A Source List of Nuclear Data Bzbliographzes. Compulations, and Evaluations. BNL-NCS-50702, Second Edition (1978).

${ }^{+}$NOTE: Requests for information should be directed to the appropriate service center. 


\section{Table 2}

\section{Other Nuclear Data Bibliographic Sources}

1. S. Ramavataram. "Recent References". 3 issues/year in Nuclear Data Sheets. Academic Press, NY.

W.B. Ewbank, et al. Nuclear Structure References. 1969-1974. Nuclear Data Sheets 16 , Supplement. Academic Press, NY (1975).

2. INIS Atomindex. International Atomic Energy Agency, Vienna.

3. Atomic Data Nuclear Data Tables 18, 1. Academic Press, NY (1976). Atomic Data Nuclear Data Tables 15, 189. Academic Press, NY (1975).

F.K. McGowan and W.T. Milner. Reaction List for Charged-Particle-Induced Reactions. Atomic Data Nuclear Data Reprints 2, Academic Piess, wY (1974).

Atomic Data Nuclear Data Tables 12, 499. Academic Press, NY (1973).

Nuclear Data Tables A11, 1. Academic Press (1972).

4. N.E. Holden and T.w. Burrows. The Bibliography uf Integral Charged Particle Nuclear Data. BNL-NCS-50640 (1982). Fourth Edition, Supplement 2.

T.W. Burrows and G. Wyant. The Bioliography of Integral Charged Particle Nuclear Data. BNL-NCS-50640 (1981). Fourth Edition, Supplement 1.

T.W. Burrows and P. Dempsey. The Biblicgraphy of Integral Charged Particle Nuclear Data. BNL-NCS-50640 (1980). Fourth Edition, (Archival Edition).

5. N.E.Holden, et al. Integral Charged Particle Nuclear Data Bibliography. BNL-NCS-51771 (1987). First Edition, Supplement 3.

N.E.Holden, et al. Integral Charged Particle Nuclear Data Bibliography. BNL-NCS-51771 (1986). First Edition, Supplement 2.

N.E.Holden, et al. Integral Charged Particle Nuclear Data Bibliography. BNL-NCS-51771 (1985). First Edition, Supplement 1.

N.E.Holden, et al. Charged Particle Nuclear Data Bibliography. BNL-NCS-51771 (1984).

First Edition.

\section{I1. SCOPE}

For the purposes of this bibliography, integral charged particle data may be defined as data which have been integrated over the product angle, and energy or data which may be easily converted to such a representation. General criteria for inclusion of data in this bibliography are the rollowing:

1. Excitation functions, thick target, or product yields $\S$ leading to the rormation of a ground or metastable state. This includes such quantities as fission yields, isomeric ratios, and excitation functions for specific particle groups, where such data can readily yield information on the excitation functions or the thick target yields for the metastable or the ground state.

2. The atomic mass and charge of the incident particle must be greater than or equal to 1.

3. The atcmic mass of the target must be greater than or equal to 1.

4. The atomic masses of the outgoing and residual nuclei must be greater than or equal to 1 with the exception of processes which do not lead to a definite residual nucleus (e.g. total cross sections) and of gamma-ray production cross sections.

§Thick target yields are defined as measurements made upon targets, whose thickness is sufficient to degrade the incident particle energy below the reaction threshold. The term product yield is employed when, in our opinion, there is an ambiguity as to what quantity has been measured. 
5. The incident particle energy must have a minimum of less than $100 \mathrm{MeV}$ in the laboratory system.

EXCEPTIONS: 1. If there are data in an article satisfying the above criteria and additional data satisfying all the criteria except minimum energy, these additional data are also cited.

\section{DATES OF COVERAGE}

The coverage for this bibliography includes all journals, reports, and other publications scanned between April 11, 1987, and November 10, 1988.

Table 3 lists the coverage of 28 "core" journals to give an indication of how current this bibliography is in ournal coverage. A complete list of the journal and report coverage may be obtained from the authors.

\section{Table 3}

\section{"Core" Journal Coverage}

\begin{tabular}{|c|c|c|}
\hline Journal & $\begin{array}{c}\text { Issue } \\
\text { Vol. }\end{array}$ & $\begin{array}{c}\text { Scann } \\
\text { No. }\end{array}$ \\
\hline Acta Phys.Pol.B & 18 & 12 \\
\hline Ann.Nucl.Energy & 15 & 9 \\
\hline Ann.Phys.(N.Y.) & 186 & 2 \\
\hline At.Energy & 63 & 6 \\
\hline Aust.J.Phys. & 41 & 2 \\
\hline Bull.Am.Phys.Soc. & 33 & 7 \\
\hline Can.J.Phys. & 66 & 6 \\
\hline Czech.J.Phys.B & 38 & 9 \\
\hline Helv.Phys.Acta & 61 & 7 \\
\hline Int.J.Appl.Radjat.Isotop. & 39 & $s$ \\
\hline Izv.Akad.Nauk SSSR, Ser.Fiz. & 52 & 7 \\
\hline J.Phys.Soc.Jpn. & 57 & 8 \\
\hline J.Phys.(Landon) $G$ & 14 & 9 \\
\hline J.Radioana1.Nucl.Chem. & 128 & 1 \\
\hline
\end{tabular}

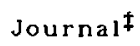

\footnotetext{
f Journal abbreviations are in agreemert with those from the Chemical Abstracts Service for 1970. See also Setzal Titles, DOE/TIC-4579-R14 (1986).
} 


\section{SECONDARY SOURCES OF INFORMATION}

Since this bibliography is designed to be both archival and current, abstracts, theses, progress reports, etc. have been included.

\section{ORGANIZATION}

This bibliography is divided into three sections, 'References', 'Target Index', and 'Residual Index'. The 'References' section contains all references satisfying the criteria noted previously and is organized as described in the next paragraph. Entries in the 'References' section are key-numbered. These keynumbers serve as a link between the three sections. The 'Target Index' section contains the incident particle energy and the abbreviated reference lines for all the entries, which contain information on a definite target nucleus and reaction. These reference lines contain the Journal name, followed by the volume and page number, as well as the keynumber of the reference. This section is described in more detail in a later paragraph. The 'Residual Index' section also contains the incident particle energy and the abbreviated reference lines for all the entries, which contain information on a definite residual nucleus, and a definite tagt-reaction, as well as the keynumber of the reference. This section is described in more detail in a later paragraph.

The 'References' section is ordered by keynumber. This keynumber is composed of two digits which correspond to he year of publication, followed by the first two letters cf the first author's name, and rompleted with a two digit running number to distinguish entries which have the same first four symbols. The reference gives all authors by name and initials, the Journal title, volume number, page number, year and the full title of the article. A Iist of keywords is given, which are designed to tell the reader what experimental results are reported in the article and what the author concluded from these results.

The 'Target Index' section contains information on those references to a particular reaction on a particular target nucleus at a given energy. The section is ordered by increasing $Z$ of the target nucleus, and by increasing $A$ of the target. For a given target the ordering is by increasing $Z$ and $A$ of the incident projectile, and finally by increasing $Z$ and $A$ of the sum of the outgoing particles. The incident energy, the abbreviated reference and keynumber complete the entry line.

The 'Residual Index' section contains information on those references to the proruction of a particular residual nucleus at a given energy. The section is ordered by increasing $Z$ and $A$ of the residual nucleus, and then by increasing $Z$ and $A$ of the incident projectile. The incident energy, the abbreviated reference and the keynumber complete the entry line.

\section{COMPILATIONS AND EVALUATIONS}

To enhance the usefulness of this bibliography several compilations, evaluations, and reviews are given in Table 4. Reference 1 contains a more comprebensive list.

\section{Table 4}

\section{Selected Compilations, Evaluations, and Reviews of}

\section{Charged-Particle Nuclear Data}

1. R.J. Howerton. Maxwell-Averaged Reaction Rates ( $\overline{\sigma v}$ ) for Selected Reactions between lons with Atomic Mass $\leq 11$. UCRL-50400 Vol. 21, Pt. A (1979).

2. K.A. Keller, et al. Q-Values and Excitation Functions of Nucleat Reactions. Landholt-Boernstein, Group $l$, Vol. 5. Parts A-C. Springer-Verlag, Berlin (1973). 
3. H. J. Kim, et al. Nuclear Cross Sections for Charged-Particle-Induced Reactions. Nucl. Data $A 3,123$. Academic Press, NY (1967).

Nucl. Data $A 2,1$. Academic Press, NY (1966).

Nucl. Data A1, 203. Academic Press, NY (1965).

4. H. Liskien and A. Paulsen. Neutron Production Cross Sections and Energies for the Reactions $T(p, n)^{3} \mathrm{He}, D(d, n)^{3} \mathrm{He}$, and $T(d, n)^{4} \mathrm{He}$. Nucl. Data Tables 11, 569. Academic Press, NY (1973). Neutron Production Cross Sections and Energies for the Reactions ${ }^{7} L 2(p, n)^{7} B e$ and ${ }^{7} L i(p, n)^{7} B e^{*}$. At. Data Nucl. Data Tables 15,57. Academic Press, NY (1975).

5. F. K. McGowan, et al. Nuclear Cross Sections for Charged-Particle Induced Reactions Mn. Fe, Co. ORNL-CPX-1 (1964).

F. K. McGowan, et al. Nuclear Cross Sections for Charged-Particle Induced Reactions Ni. Cu. ORNL-CPX-2 (1964).

6. H. Muenzel, et al. Karlsruhe Charged Particle Reaction Data Compilation.

Physik Daten 15-5 (1982).

Physik Daten 15-4 (1982).

Physik Daten 15-3 (1982).

Physik Daten 15-2 (1979).

Physik Daten 15-1 (1979).

Physik Daten 15-Index (1979).

7. W. Neubert. Peak Cross Sections for (Hl,xn) Reactions. Nucl. Data Tables A11, 531. Academic Press, NY (1973).

8. S. Pearlstein. Starter Evaluated Charged Particle Data Library. BNL-19148 (1974).

9. S.T. Perkins and D.E. Cullen. Experimental and Evaluated Nuclear Plus Interference Cross Sections for Light Charged Particles. Nucl. Sci. Eng. 77(1), 20 (1981). ibid. UCRL-50400 Vol. 15, Pt. F.

\section{METHODS OF RADIOISOTOPE PRODUCTION}

While this publication should be very useful in provicing the sources of cross section data for radioisotope production, its scope does not include the incexing of techniques for radioisotope or labelled-compound production. The Radiopharmaceutical Research Group, Chemistry Department, Brookhaven National Laboratory does index such techniques for accelerators. 2

\section{RETRIEVAL CAPABILITIES}

Retrievals may be obtained from this Charged-Particle Bibliography using the Nuclear Structure References rile, based on target nucleus, incident particle, outgoing particles or reactions, residual nuclide, topic, or any combination of these. The term topic refers to the jntegral cross section, $(\sigma)$, the thick target yield, (TTY), or the product yield, (yield). Additional criteria are lists of keynumbers of the references, publication date, and entry date. Send requests to:

National Nuclear Data Center

Building $197 \mathrm{D}$

Upton, NY 11973

U.S.A.

2 D.R. Christman and K.I. Karlstrom. Accelerator Produced Nuclides for Use in Biolugy and Medicine. A Bibliography, 1939-1973. BNL-50448 (1975).

K.I. Karlstrom and D.R. Christman. Accelerator Produced Nuclides for Use in Biology and Medicine. A Bibliography, January 1974 - June 1976. BNL-50448-Vol. II (1978).

K.l. Karlstrom and D.R. Christman. Accelerator Produced Nuclides for Use in Biology and Medicine. A Bibliography, July 1976 - December 1980. BNL-50448-Vol. III (1983). 
For more detailed information, contact the authors.

All Information Analysis Centers supported by the Department of Energy are required to conform with a DOE plan for charging services. There are no charges to the DOE and its contractors; however, there could be nominal charges for others. Further information will be supplied upon request.

\section{ACKNOWLEDGEMENTS}

The charged-particle activities at the National Nuclear Data Center have been supported by the Orfice of Basic Energy Sciences, U.S. Department of Energy. 
$85 \mathrm{Ja} 16$

N.Jarmie, R.E.Brown - Nucl.Instrum.Methods Phys.Res. B10/11, 405 (1985) Reactions with Hydrogen Isotopes NUCLEAR REACTIONS ${ }^{2} \mathrm{H}(\mathrm{d}, \mathrm{p}),\left(\mathrm{d},{ }^{3} \mathrm{He}\right), \mathrm{E}=20-117 \mathrm{keV}$; measured $\sigma ;$ deduced $\sigma(\mathrm{E})$ for ${ }^{2} \mathrm{H}\left(\mathrm{d},{ }^{3} \mathrm{He}\right)$, astrophysical S-function rs $E$.

86Aj04 A.Ajt Haddou, M.Berrada, G.Paic - J.Radioanal.Nuct.Chem. 102, 159 (1986) - Yields and Ayerage Cross Sections of Recoil Charged Particle Induced Reactions on ${ }^{11} \mathrm{~B},{ }^{12} \mathrm{C},{ }^{13} \mathrm{C},{ }^{14} \mathrm{~N},{ }^{10} \mathrm{O}$ and ${ }^{28} \mathrm{O}$ NUCLEAR REACTIONS ${ }^{11} \mathrm{~B},{ }^{13} \mathrm{C},{ }^{18} \mathrm{O}(\mathrm{p}, \mathrm{n}),{ }^{12} \mathrm{C}(\mathrm{p}, \gamma),{ }^{12} \mathrm{C},{ }^{16} \mathrm{O}(\mathrm{d}, \mathrm{n}),{ }^{14} \mathrm{~N},{ }^{1 \theta} \mathrm{O}(\mathrm{p}, \alpha),{ }^{16} \mathrm{O}(\mathrm{t}, \mathrm{n}), \mathrm{E}<\mathrm{l4.7} \mathrm{MeV}$ measured o,residuals yields. Nal detectors,activation method.

$86 \mathrm{Ba} 84$

V.G.Batti, E.A.Skakun, Yu.N.Rakjvnenko, O.A.Rastrepin! - Izv.Akad.Nauk SSSR, Ser.Fiz. 50, 913 (1986); Bull.Acad.Sci.USSR, Phys.Ser. 50, No.5, 80 (1986) - Identifying the Excitation Cross Section of a ${ }^{100 \mathrm{~m}} \mathrm{Ta}\left(\mathrm{T}_{1 / 2} \leq 10^{13}\right.$ Years $)$ isomer in a $(\mathrm{p}, n)$-Heaction NUCLEAR REACTIONS ${ }^{378,180} \mathrm{Hr}(p, n), \mathrm{E} \leq 9.1 \mathrm{MeV}$; measured residual production $\sigma(\mathrm{E}) .{ }^{180} \mathrm{Hf}(\mathrm{p}, \mathrm{n})$, $E=6-9$ MeV; evaluated residual production $\sigma(E)$. Ge(Li) detector.

$86 K 033$

1.0.Konstantinov, P.P.Dmitriev, V.I.Bolotsky - At.Energ. 60, 332 (1966); Sov.At.Energy 60, 390 (1986) - Actjuation of Zirconium,Niobium, and iantalum in a Cyclotron NUCLEAR REACTIONS Zr,Nb,Ta(p,X),E=6.1-22.4 MeV; Zr,Nb,Ta(d,X),E=6.2-22.6 MeV; Zr,Nb,Ta(a $\mathrm{X})^{92} \mathrm{Nb} /{ }^{92 \mathrm{~m}} \mathrm{Nb} /{ }^{91} \mathrm{Nb} /{ }^{91 \mathrm{~m}} \mathrm{Nb} /{ }^{80} \mathrm{Y} /{ }^{85} \mathrm{Nb} /{ }^{95} \mathrm{Zr} /{ }^{89} \mathrm{Zr} /{ }^{95} \mathrm{Tc} /{ }^{95 \mathrm{~m}} \mathrm{Tc} /{ }^{182} \mathrm{Ta} /{ }^{181} \mathrm{~W} /{ }^{183} \mathrm{Re} /{ }^{184} \mathrm{Re} /{ }^{181} \mathrm{Ta} /{ }^{87} \mathrm{Y}, \mathrm{E}=12.6-45.2$ MeV; measured residual yields vs E. Zr,Nb,Ta(p,X),E=11,13,21-22.6 MeV; Zr,Nb,Ta(d,X), $\mathrm{E}=12,20-45.2 \mathrm{MeV} ; \mathrm{Zr}, \mathrm{Nb}, \mathrm{Ta}(\alpha, \mathrm{X})^{92} \mathrm{Nb} /{ }^{92 \mathrm{~m}} \mathrm{Nb} /{ }^{91} \mathrm{Nb} /{ }^{91 \mathrm{~m}} \mathrm{Nb} /{ }^{80} \mathrm{Y} /{ }^{95} \mathrm{Nb} /{ }^{95} \mathrm{Zr} /{ }^{89} \mathrm{Zr} /{ }^{95} \mathrm{Tc} /{ }^{95 \mathrm{~m}} \mathrm{Tc} /{ }^{182} \mathrm{Ta} /{ }^{16 \mathrm{~W}} /$ ${ }^{183} \mathrm{Re} /{ }^{184} \mathrm{Re} /{ }^{181} \mathrm{Ta} /{ }^{87} \mathrm{Y} 5=11,18,26,41-46 \mathrm{MeV}$; compiled residual yields.

$862 a 13$

P.P.Zarubin, V.P.Gusey, A.E.Antropov - lzv.Akad.Nauk SSSR, Ser.Fiz. 50, 160 (19B6): Bull.Acad.Sci.USSR, Phys.Ser. 50, No.1, 156 (1986) - A Threshold Isotopic Esfect in Competing Proton Scattering Mechanisms at Epibarrier Energies for Nuclides of Medium Atomic Weight NUCLEAR REACTIONS Mg,Si,S,Ti,Cr,Fe,Ni,Zn,Mo,Ru,Pd(p,p),(p,ii),E=6 MeV; compiled reaction Q,excitation energies, $\sigma$; deduced compound nucleus contribution.

B62hog B.V.Zhuravlev, S.P.Ivanova, N.N.Krasnov, Yu.N.Shubin - At.Energ. 60, 337 (1986); Sov.At.Energy 60, 395 (1986) - Production of ${ }^{123}$ I by lrradiating 124 Xe with 20-30-MeV Protons

NUCLEAR REACTIONS $12+\mathrm{Xe}(p, 2 n),(p, n p),(p, \alpha),(p, 2 p),(p, 2 n p), 126 x e(p, 2 \jmath),(p, 2 p),(p, \alpha),(p, n p)$, $(p, n \alpha),(p, n 2 p),{ }^{129} \mathrm{Xe}(p, n \alpha),{ }^{123} \mathrm{Xe}(p, \alpha), \mathrm{E} \approx 15-30 \mathrm{MeV}$; calculated reaction o(E). Statistical theary

87Ab15 S.N.Abramovich, B.Ya.Guzhovsky, A.V.Ershov, L.M.Lazarev - Yad.Fiz. 46, 499 (1987) - Analysis of Anomalies in the Excitatios Function for the ${ }^{7} \mathrm{Li}(\mathrm{t}, \mathrm{p})^{9} \mathrm{Li}$ Reaction NUCLEAR REACTIONS ${ }^{7} \mathrm{Li}(\mathrm{t}, \mathrm{p}),(\mathrm{t}, \mathrm{n}), \mathrm{E} \approx 5.7-\mathrm{B} .5 \mathrm{MeV}$; analyzed $\sigma(\mathrm{E})$. ${ }^{10} \mathrm{Li}$ ded uced binding energy. ${ }^{10} \mathrm{Be}$ deduced Jevels, $\Gamma$. General suclear reaction theory.

87Ag05 C.E.Agujar, V.C.Barbosa, L.F.Canto, R.Donangelo - Nucl.Phys. A472, 571 (1987) - Liguid-Drop Model Description of Heavy lon Fusion at Sub-Barrier Energies NUCLEAR REACTIONS ${ }^{58} \mathrm{Ni}\left({ }^{58} \mathrm{Ni}, \mathrm{Y}\right), \mathrm{E}(\mathrm{cm}) \approx 92-110 \mathrm{MeV} ;{ }^{64} \mathrm{Ni}\left({ }^{84} \mathrm{Ni}, \mathrm{X}\right), \mathrm{E}(\mathrm{cm}) \approx 90-105 \mathrm{MeV}$; ${ }^{40} \mathrm{Ca}\left({ }^{40} \mathrm{Ca}, \mathrm{X}\right), \mathrm{E}(\mathrm{cm}) \approx 50-65 \mathrm{MeV} ;{ }^{74} \mathrm{Ge}\left({ }^{74} \mathrm{Ge}, \mathrm{X}\right), \mathrm{E}(\mathrm{cm}) \approx 110-150 \mathrm{MeV} ;{ }^{90} \mathrm{Zr}\left({ }^{90} \mathrm{Zr}, \mathrm{X}\right), \mathrm{E}(\mathrm{cm}) \approx$ 172-195 MeV; ${ }^{\circ 0} \mathrm{Se}\left({ }^{\circ 0} \mathrm{Se}, \mathrm{X}\right), \mathrm{E}(\mathrm{cm}) \approx 130-150 \mathrm{MeV} ;$ calculated rusion o(E). Liquid drop model.

87Ag06 V.A.Ageev, V.Ya.Golovnya, E.A.Gromova, S.A.Egorov, S.S.Kovalenko, A.F.Linev. Yu.A.Nemilov, A.V.Pozdnyakov, V.A.Rubchenya, Yu.A.Selitsky, A.M.Fridkin, V.B.Funshtein, V.A.Yakovlev Yad.Fiz. 46, 700 (1987); Sov.J.Nucl.Phys. 46, 392 (1987) - Cross Sections for the Reaction ${ }^{238}$ (p) $\mathrm{xn})(239-\mathrm{x}) \mathrm{Np}$ in the Proton-Energy Range from 7 to $30 \mathrm{MeV}$

NUCLEAR REACTIONS ${ }^{238} \mathrm{U}(\mathrm{p}, \mathrm{n}),(\mathrm{p}, 3 \mathrm{n}), \mathrm{E} \approx 10-40 \mathrm{MeV}$ : measured $\sigma(\mathrm{E}),{ }^{230} \mathrm{~Np}$ isomer production $\sigma$ ratio. ${ }^{2 j \theta} U(p, n),(p, 2 n),(p, 3 n), E \approx 10-40$ MeV; calculated o(E). Statistical model. Preequilibrium particle emission.

87Aj01 N.N.Ajitanand, K.N.1yengar, R.P.Anand, D.M.Nadkarni, A.K.Mohanty - Phys.Rey.Lett. 58, 1520 (1987) - Anomalous Behavior of the Proton-Induced Fision Cross Sections of ${ }^{235} U$ and ${ }^{238} U$ at Extreme Sub-Barrier Energies

NUCLEAR REACTIONS ${ }^{235}{ }^{23 B} U(p, F), E=0.5-4.3$ MeV; measured rission $\sigma(E)$. Enriched $235 U, n a t u r a l$ Uranium targets,solid state track detectors

87 Al29 V.N.Aleksandrov, M.P.Semenova, V.G.Semenov - At.Energ. 62, 411 (1987); Sov.At.Energy 62, 478 (1987) - Production Cross Section of Radionuclides in (,$x$ ) Reactions at Copper and Nickel Nuclei NUCLEAR REACTIONS $\mathrm{Cu}(\mathrm{p}, \mathrm{X})^{98} \mathrm{Co} /{ }^{81} \mathrm{Cu} /{ }^{82} \mathrm{Zn} /{ }^{63} \mathrm{Zn} /{ }^{38} \mathrm{Co} /{ }^{57} \mathrm{Co} /{ }^{60} \mathrm{Cu} /{ }^{59} \mathrm{Fe}, \mathrm{Ni}(\mathrm{p}, \mathrm{X})^{58} \mathrm{Co} /{ }^{57} \mathrm{Co} /{ }^{57} \mathrm{Ni} /{ }^{96} \mathrm{Co} /$ ${ }^{56} \mathrm{Ni} /{ }^{91} \mathrm{Cr} /{ }^{52} \mathrm{Mn} /{ }^{60} \mathrm{Cu}, \mathrm{E}=10-100 \mathrm{MeV}$; measured residual production $\sigma(E)$. Activation technique.

87fr13 A.E.Aravantinos, A.C.Xenoulis - Phys.Rev. C35, 1743 (1987) - Reactjon Mechanism of pn ard d Emission in Certain Heavy-lon-Induced Nuclear Reactions 
NUCLEAR REACTIONS ${ }^{12} \mathrm{~L},{ }^{28} \mathrm{SI}\left({ }^{12} \mathrm{C}, \mathrm{np}\right),\left({ }^{12} \mathrm{C}, \mathrm{d}\right), \mathrm{E}(\mathrm{cm})=13.8-22.1 \mathrm{MeV} ;{ }^{6} \mathrm{Li},{ }^{16} \mathrm{O},{ }^{30} \mathrm{Si}\left({ }^{6} \mathrm{Li}, \mathrm{np}\right),\left({ }^{8} \mathrm{Li}, \mathrm{d}\right)$, $\mathrm{E}(\mathrm{cm})=7.2-13.3 \mathrm{MeV} ;{ }^{12} \mathrm{C}\left({ }^{2} \mathrm{Li}, \mathrm{d}\right),\left({ }^{12} \mathrm{C}, \mathrm{np}\right), \mathrm{E}(\mathrm{cm})=7.4 .9 .4 \quad \mathrm{MeV} ;{ }^{12}{ }^{13} \mathrm{C},{ }^{16} \mathrm{O}\left({ }^{16} \mathrm{O}, \mathrm{np}\right),\left({ }^{16} \mathrm{O}, \mathrm{d}\right)$. F(cm; $=11-16 \mathrm{MeV}$; calculated (np),d emission oresidual production $\sigma(E)$ ratio. Hauser-Feshbach theory.

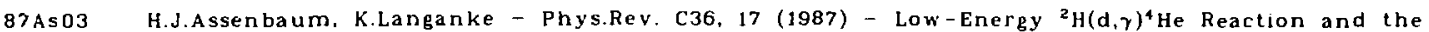
$D$-State Admixture in the ${ }^{4} \mathrm{He}$ Ground State

NUCLEAR REACTIONS ${ }^{2} \mathrm{H}(\mathrm{d} . \gamma), \mathrm{E}(\mathrm{cm})=0.010-10 \mathrm{MeV}$; calculated $\sigma($ Er.E).capture $\sigma . a s t r o p h y s i c a l$ $S-f a c t o r$ vs E. "He level dedured D-state adm:xture.

87 A 05

H.d.Assenbaum, K.Langanke, C.Rolfs - 2.Phys, A327, 461 (1987) - Erfects of Electron Screening of Low-Energy Fusion Cross Sections

NUCLEAR REACTIONS ${ }^{2} \mathrm{H},{ }^{3} \mathrm{He}(\mathrm{d}, \mathrm{p}), \mathrm{E} \leq 50 \mathrm{keV} ;{ }^{3} \mathrm{He}\left({ }^{3} \mathrm{He}, 2 \mathrm{p}\right),{ }^{2} \mathrm{Li},{ }^{18} \mathrm{O},{ }^{11} \mathrm{~B},{ }^{15} \mathrm{~N}(\mathrm{p}, \alpha), \mathrm{E} \leq 250 \mathrm{keV}$; calculated reaction $\sigma$,astrophysical $S(E)$ factors. Electron screening effects.

87Ba5I G.J.Balster, R.H.Siemssen, H.W.Wilschut - Nucl.Phys. A471, 635 (198\%) - Systematics of the 14N + ${ }^{159} \mathrm{~Tb}$ Reaction between 6 and $22 \mathrm{MeV} / \mathrm{u}$ : The origin of fast a-particles NUCLEAR REACTIONS ${ }^{159} \mathrm{~Tb}\left({ }^{14} \mathrm{~N}, \alpha \mathrm{aX}\right), \mathrm{E}=92.112,115,140,168,236,309 \mathrm{MeV} ;$ measured (fragment) $(\mathrm{K}$ $X$-ray)coln; deduced $\sigma(E), \sigma(\alpha)(Z($ res $))$ decomposition.

$87 \mathrm{Ba} 53$

F.C.Barker - Aust.J.Phys. $40,25(1987)$ - The ${ }^{12} \mathrm{C}(\alpha, \gamma)^{16} \mathrm{O}$ Cross Section at Stellar Energies NUCLEAR REACTIONS ${ }^{12} \mathrm{C}(a, \gamma), \mathrm{E}(\mathrm{cm}) \approx 1.5-3 \mathrm{MeV} ;$ calculated $\sigma(E) ;$ deduced dipole contribution at stellar energies.

$87 \mathrm{Ba} 58$

C.A.Barnes, K.H.Chang. T.R.Donoghue, C.Rolfs, J.Kammeraad - Phys.Lett. 197B, 315 (1987) - The

${ }^{2} \mathrm{H}(\mathrm{d}, \gamma)^{4} \mathrm{He}$ Reaction at Low Energy and the D-State Admixture in "He NUCLEAR REACTIONS ${ }^{2} \mathrm{H}(\mathrm{d}, \gamma), E(\mathrm{~cm})=50-500 \mathrm{keV}$; measured $\sigma(\theta), \sigma(E)$; deduced dominant radiative capture amplitude character,astrophysical S-factor vs E. "He deduced $\mathrm{C}$-State admixture.

87Bagl S.v.Bakhmutkin, A.l.Bogdanov, V.G.Bogdanov, N.P.Kocherov, V.I.Kucheryuk, A.A.Nosov, A.A.Rimsky-Korsakov - At.Energ. 62, 59 (1987); Sov.At.Energy 62, 81 (1987) - Energy Spectra of Neutrons on the Surface of ? Cylindrical Lead Target Irradiated with 1-GeV Protons NUCLEAR REACTIONS $P b(p, x n), E=1$ GEV; measured neutron spectra. Activation technique. ${ }^{197} \mathrm{~A} \mathrm{u}(\mathrm{n}, \gamma), \mathrm{E}=0.1-1 \mathrm{MeV} ;{ }^{197} \mathrm{Au}(\mathrm{n}, 2 \mathrm{n}), \mathrm{E}=9.50 \mathrm{MeV} ;{ }^{197} \mathrm{Au}(\mathrm{n}, 3 \mathrm{n}), \mathrm{E}=15-50 \mathrm{MeV} ;{ }^{197} \mathrm{Au}(\mathrm{n}, 4 \mathrm{n})$, $E=25-50 \mathrm{MeV} ;$ measured $\sigma(E) .{ }^{59} \mathrm{Co}(n, 2 n), E \leq 50 \mathrm{MeV} ;{ }^{59} \mathrm{Co}(\mathrm{p}, \mathrm{np}), \mathrm{E} \approx 12.5-50 \mathrm{MeV} ;{ }^{59} \mathrm{Co}(\mathrm{n}, 3 \mathrm{n})$, $\mathrm{E} \approx 25-50 \mathrm{MeV} ;{ }^{50} \mathrm{Co}(\mathrm{p}, 2 \mathrm{np}), \mathrm{E} \approx 25-50 \mathrm{MeV} ;$ measured residual production $\sigma(E)$. Activation technique.

87Be17 H.W.Becker, C.Rolfs, H.P.Trautvetter - Z.Phys. A327, 341 (1987) - Low-Energy Cross Sections for ${ }^{11} B(p, 3 a)$

NUCLEAR REACTIONS "B $\mathrm{B}(\mathrm{p}, 3 \alpha), \mathrm{E}(\mathrm{cm})=22-1100 \mathrm{keV}$; measured Ea,la, $\sigma(\mathrm{E}, \theta), \sigma\left(\mathrm{E}, \theta\left(\alpha_{1}\right), \theta\left(\alpha_{2}\right)\right)$, $\alpha-y$ ield vs $E \alpha .{ }^{11} \mathrm{~B}(\mathbf{p}, \alpha), E=140-1100 \mathrm{keV}$; measured $\alpha$ yiejd vs $\theta, \sigma(\theta) .{ }^{1} \mathrm{H}\left({ }^{11} \mathrm{~B}, \alpha\right), \mathrm{E}=1.68-13.2$ $\mathrm{MeV}$; measured $\sigma(E)$; deduced absolute $\sigma$,astrophysical $S(E)$ factor. ${ }^{12} \mathrm{C}$ deduced resonance, $\Gamma$. Solid,windowless gas targets,Si detectors,kinematically complete experiment.

87Bio3 B.Bilwes, R.Bilwes, J.Diaz, J.L.Ferrero, D.Pocanic, L.Stuttge - Nucl.Phys. A463, 731 (1987) Intermediate Structure in the ${ }^{2 \theta} \mathrm{Si}+{ }^{32} \mathrm{~S}$ System

NUCLEAR REACTIONS ${ }^{28} \mathrm{Si}\left({ }^{32} \mathrm{~S},{ }^{32} \mathrm{~S}\right),\left({ }^{32} \mathrm{~S},{ }^{36} \mathrm{Ar}\right), \mathrm{E}=42-48.1 \mathrm{MeV}$; measured $\sigma(\theta), \sigma(E)$; deduced deviation,correlation functions. Optical-model calculations. Enriched targets.

87Bi21 A.M.Bizzeti-Sona, P.Blasi, A.A.Stefanini, A.J.Krejner - Phys.Rev. C36, 2330 (1987) - High Spin States in ${ }^{80} \mathrm{Tc}$ via the ${ }^{94} \mathrm{Zr}\left({ }^{7} \mathrm{Li}, 3 \mathrm{n}\right)^{98} \mathrm{Tc}$ Reaction

NUCLEAR REACTIONS ${ }^{94} \mathrm{Zr}\left({ }^{7} L i, 3 n\right), E=23-31$ MeV; measured E $\gamma, 1 \gamma, \gamma \gamma$-coin, $\gamma$ yield vs E, $1 \gamma(\theta)$,

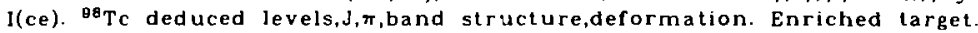

$87 \mathrm{~B} \mid 12$

G.Bluge, H.J.Assenbaum, K.Langanke - Phys.Rev. C36, 21 (1987) - Potential Model Analysis of Low Energy ${ }^{2} H(d, \gamma)^{4}$ He Fusion Data

NUCLEAR REACTIONS ${ }^{2} \mathrm{H}(\mathrm{d}, \gamma), \mathrm{E}(\mathrm{cm})=0.010-10 \mathrm{MeV}$; calculated $\sigma(E \gamma$, E).capture $\sigma$,astrophysical $S$-factor vs E,phase shifts vs E. "He level deduced D-state adinixture nucleus-nucleus potential dependence.

87Bo16 N.Bordes, G.Blondiaux, C.J.Maggiore, M.Valladon, J.L.Debrun, R.Coquille, M.Gauneau Nucl.instrum.Methods Phys.Res. B24/25, 72? (1987) - Analytical Possibilities of Medium Energy Tritium Beams $(3<E<12 \mathrm{MeV})$ and Application to the Analysis of $0 x y$ gen in inP NUCLEAR REACTIONS ${ }^{10} 0,{ }^{26} \mathrm{Si},{ }^{32} \mathrm{~S}(\mathrm{t}, \mathrm{n}),{ }^{27} \mathrm{Al},{ }^{25},{ }^{26} \mathrm{Mg}{ }^{71} \mathrm{Ga}(\mathrm{t}, \rho),{ }^{10} \mathrm{~B}(\mathrm{t}, 2 \mathrm{n}),{ }^{73} \mathrm{As}(\mathrm{t}, \mathrm{d}), \mathrm{E}=3-32 \mathrm{MeV}$ measured thick target yields $v s E_{;}$deduced activation analysis sensitivities. Ge(Li) detector. 
$87 \mathrm{BrOZ}$

87 Br 10

87Bu 16

$87 \mathrm{Bu} 18$

$87 \mathrm{Ca} 33$

A.Calboreanu, o.Salagean, C.Pencea, K.w.2immer, A.Ciocanel - Rev.Roum.Phys. 32, 725 (1987) Formation and Decay of the Compound Nucleus in Alpha lnduced Reaction on ${ }^{70} \mathrm{Ge}$ NUCLEAR REACTIONS ${ }^{70} \mathrm{Ge}(\alpha, n),(\alpha, 2 n), \mathrm{E}=2 \mathrm{hreshold-24} \mathrm{MeV}$; measured $\sigma(E)$; deduced mode] parameters. ${ }^{74}$ Se deduced formation $\sigma$, level density parameter.

$87 \mathrm{ChO4}$

R.K.Choudhury, A.Saxena, V.S.Ramamurthy, D.M.Nadkarni, S.S.Kapoor - Nucl.Phys. A.63, 597 (1987) - Variation of Kinetic Energy Distributions of Fission Fragments with Bombarding Energy in the Induced Fision of $23{ }^{8} \mathrm{U}$ at Medium Energies NUCLEAR REACTIONS ${ }^{238} \mathrm{U}(\alpha, F), \mathrm{E}=32,40,48,56 \mathrm{MeV}$; measured fission fragment yieId vs mass, kinetic energy distributions.

$8>\operatorname{Ch} 30$

R.M.Chasteler, R.A.Henderson, D.Lee, K.E.Gregorich, M.J.Nurmia, R.B.Welch, D.C.Horfman Phys.Rev. C36, 1820 (1987) - Excitation Functions for Production or Heavy Actinides from Interactions of ${ }^{16} \mathrm{O}$ with ${ }^{248} \mathrm{Cr}$ NUCLEAR REACTIONS ${ }^{249} \mathrm{Cr}\left({ }^{16} \mathrm{O}, \mathrm{X}\right)^{244} \mathrm{Bk} /{ }^{245} \mathrm{Bk} /{ }^{248} \mathrm{Bk} /{ }^{248} \mathrm{Bk} /{ }^{240 \mathrm{~m}_{\mathrm{Bk}} /{ }^{247} \mathrm{Cf} / 248} \mathrm{Cr} /{ }^{248} \mathrm{Es} /{ }^{249} \mathrm{Es} /{ }^{250} \mathrm{Es} /{ }^{251} \mathrm{Es} /$ ${ }^{253} \mathrm{Es} /{ }^{250} \mathrm{Fm} /{ }^{251} \mathrm{Fm} /{ }^{252} \mathrm{Fm} /{ }^{253} \mathrm{Fm} /{ }^{254} \mathrm{Fm} /{ }^{256} \mathrm{Fm}, \mathrm{E}=90,96,106,115,122,139,150$ MeV; measured residual production $\sigma(E)$,isotopic distributions.

$87 \operatorname{co} 36$

P.D.Cottle, M.Gai, J.F.Ennis, J.F.Shriner,Jr., D.A.Bromley, C.W.Beausang, L.Hildingsson, W.F.Piel,Jr., D.B.Fossan, J.W.olness, E.K.Warburton - Phys.Rev. C36. 2286 (1987) - Level Spectrum or ${ }^{210} \mathrm{Ra}$ and Weak Coupling in the Ljght Actinide Region

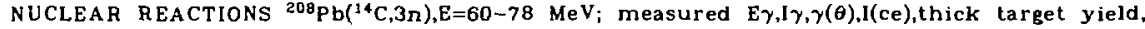

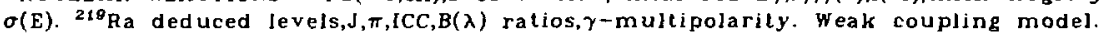

87De13 P.Descouvemont, D.Baye - Phys.Rev. C36, 54 (1987) - Mjcroscopic Theory of the BBe( $\left.{ }^{8},\right)^{12} \mathrm{C}$ Reaction in a Three-Cluster Model

NUCLEAR REACTIONS ${ }^{8} \mathrm{Be}(\alpha, \gamma), \mathrm{E}(\mathrm{cm})=0.0-4 \mathrm{MeV}$; calculated capture $\sigma$,astrophysical S-ractor, reaction rate. ${ }^{\theta} \mathrm{Be}(\alpha, \alpha), \mathrm{E}<6 \mathrm{MeV}$; calculated phase shirts. ${ }^{2} \mathrm{C}$ deduced resonances. Generator coordinate method,three-cluster model.

87De32 P.Descouvemont, D.Baye - Phys.Rev. C36, 1249 (1987) - 12C(a.r) 160 Reaction in a Multiconfiguration Microscopic Model

NUCLEAR REACTIONS ${ }^{12} \mathrm{C}(\alpha, \gamma), \mathrm{E} \approx 0.25-3 \mathrm{MeV}$; calculated $\sigma(\mathrm{E})$; deduced S-ractor vs E,E1,E2 component contribution: Microscopic generator coordinate method.

87De38 P.Descouvemont - Phys.Rev. C36, 2206 (1987) - Microscopic Analysis of the ${ }^{13} C(\alpha, n)^{18} 0$ and ${ }^{13} C(\alpha$, a) ${ }^{13} \mathrm{C}$ Reactions

NUCLEAR REACTIONS ${ }^{13} \mathrm{C}(\alpha, n),(\alpha, \alpha), \mathrm{E}=$ low; calculated transfer,alastic $\sigma(\theta), \sigma(E)$. Generator corrdinate method.

87Di07 Th.Diacc, C.Friedit, P.Lerch - Radiochim.Acta 42, 1 (1987) - Total Cross-Sections ror Reactions Induced by Oxygen-18 on Lithium

NUCLEAR REACTIONS ${ }^{8} \mathrm{Li}\left({ }^{18} \mathrm{O}, \mathrm{p}\right),\left({ }^{18} \mathrm{O}, \alpha\right),\left({ }^{18} \mathrm{O}, 2 \alpha\right),\left({ }^{18} \mathrm{O},{ }^{3} \mathrm{He}\right),\left({ }^{10} \mathrm{o}, n\right),\left({ }^{18} \mathrm{O}, \mathrm{p}\right),\left({ }^{18} \mathrm{O}, \mathrm{d}\right),\left({ }^{18} \mathrm{O}, \mathrm{na}\right),\left({ }^{18} \mathrm{O}\right.$, $\mathrm{pa}), \mathrm{E}=10-40 \mathrm{MeV}$; measured $\sigma(\mathrm{E})$,yields. Activation technique. 
87Do05

87 Do06

87EnO2

$87 \mathrm{FiOB}$

$87 \mathrm{Fi} 1$

D.Fink, M.Paul, G.Hollos, S.Theis, S.Vogt, R.Stueck, P.Englert, R.Michel - Nucl.1nstrum.Methods Phys.Res. E29, 275 (1987) - Measurements of ${ }^{41} \mathrm{Ca}$ Spallation Cross Sections and 4 Ca Concentrations in the Grant Meteorite by Accelerator Mass Spectrometry NUCLEAR REACTIONS Ni.Fe,Ti(p,X)"Ca,E=40-600 MeV; measured residual spallation production $v(\mathrm{E})$.

87FrI 1

$87 \mathrm{GaD3}$

D.L.Gay, N.R.fletcher, L.C.Dennis - Phys.Rev.Lett, 5B, 1512 (1987) - Resonant Fluctuations in Heavy-Ion Reaction Cross Sections: ${ }^{16} \mathrm{O}\left({ }^{16} \mathrm{O}, a\right)^{26} \mathrm{Si}$ NUCLEAR REACTIONS ${ }^{16} \mathrm{O}\left({ }^{16} \mathrm{O}, \alpha\right), \mathrm{E}=12-20 \mathrm{MeV}$; calculated $\sigma(E), \sigma(\theta)$. Compound nucleus decay model.

$87 G a 15$

M.Gai, R.Keddy, D.A.Bromley, J.W.Olness, E.K.Warburton Spectroscopy of ${ }^{10} \mathrm{O}$. Radiative capture, ${ }^{14} \mathrm{C}\left(a_{1} \gamma\right)^{18} \mathrm{O}$ NUCLEAR REACTIONS ${ }^{14} \mathrm{C}(\alpha, \gamma), \mathrm{E}=1.7-2.7$ MeV; measured $\gamma$ yield vs E. ${ }^{10} \mathrm{O}$ deduced resonances, $B(\lambda), \gamma$-multipolarity, $\gamma$-branching, $\Gamma, \Gamma, \Gamma \alpha / \Gamma, \alpha-$ reduced $w i d t h s$.

B7G 105

Yu.A.Glukhov, A.S.Demyanova, A.A.Oglablin, S.B.Sakuta - Yad.Fiz. 45, 1236 (1987) - Reaction ( ${ }^{7} L i$, ${ }^{2} \mathrm{Be}$ ) on Medium and Heavy Nuclei at the Beam Energy of $78 \mathrm{MeV}$ NUCLEAR REACTIONS ${ }^{27} \mathrm{Al},{ }^{28} \mathrm{Si},{ }^{58,60}, 62,{ }^{44} \mathrm{Ni},{ }^{80},{ }^{92},{ }^{94} \mathrm{Zr},{ }^{107},{ }^{100} \mathrm{Ag},{ }^{107} \mathrm{Au},{ }^{200} \mathrm{~Pb}\left({ }^{7} \mathrm{Li},{ }^{7} \mathrm{Be}\right) . \mathrm{E}=78 \mathrm{MeV}$ :

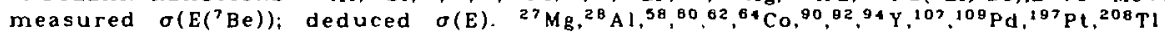
deduced particle-hole excitation gross structure. $\triangle E-E$ telescopes.

$87 \mathrm{Go} 30$

S.S.Godre, Y.R.Waghmare - Phys.Rev. C36, 1632 (1987) - Classical Microscopic Calculations of s6o $+{ }^{10} \mathrm{O}$ and ${ }^{10} \mathrm{Ca}+{ }^{10} \mathrm{Ca}$ Fusion Cross Sections

NUCLEAR REACTIONS ${ }^{16} \mathrm{O}\left({ }^{16} \mathrm{O}, \mathrm{X}\right), \mathrm{E}(\mathrm{cm}) \approx 12-100 \mathrm{MeV} ;{ }^{40} \mathrm{Ca}\left({ }^{40} \mathrm{Ca}, \mathrm{X}\right), \mathrm{E}(\mathrm{cm}) \approx 62-250 \mathrm{MeV}$; calculated fusion $\sigma(E)$. Classical microscopic approach.

87Gr33 O.V.Grusha, S.P.Ivanova, S.Yu.Platonov, O.A.Yuminov - Jzv.Akad.Nauk SSSR, Ser.Fiz. 51. 2055 (1987) - Isomeric Effect of Excited Nucleus on Evaporative Neutron Yield in the Reaction asBifd, $x n r)$ 
87Gu25

$87 \mathrm{Ha} 24$

87 Ho2?

871 t03

87 I tOB

87โพ02

87 Ja 13

$87 \mathrm{Kil}$

$87 \mathrm{~K}$ i 19

J.A.Kittl, J.E.Testoni, A.O.Macchiavelli, A.J.Pacheco, D.Abriola, D.E. Di Gregorio, A.Etchegoyen, M.C.Etchegoyen, J.o.Fernandez Niello, A.M.J.Ferrero, S.Gil - Nucl.Phys. A471. 587 (1987) Scattering,Fusion and Transfer Reactions in ${ }^{16} \mathrm{O}+(\mathrm{A})(\mathrm{Sm})$ at Energies Close to the Coulomb Barrier NUCLEAR REACTIONS ${ }^{144} \mathrm{Sm}\left({ }^{16} \mathrm{O}, \mathrm{X}\right),\left({ }^{18} \mathrm{O},{ }^{16} \mathrm{O}\right),\left({ }^{16} \mathrm{O},{ }^{16} \mathrm{O}\right), \mathrm{E}=61-72,3 ;{ }^{148} \mathrm{Sm}\left({ }^{16} \mathrm{O}, \mathrm{X}\right),\left({ }^{16} \mathrm{O},{ }^{16} \mathrm{O}\right),\left({ }^{16} \mathrm{O}\right.$,

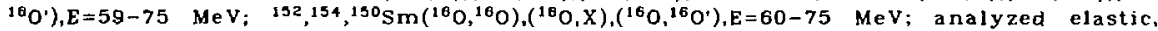
inelastic $\sigma(\theta)$ fusion,transfer $\sigma(E)$. Intrinsic coordinate dependent phase shifts, equivalent spheres formrlism.

87Ki2 1

B.T.Kim - Phys.Rev. C36, 1626 (1987) - Dynamic Polarization Potential ror Coupled-Channels Effects on the Heavy-Ion Fusion Reactions NUCLEAR REACTIONS ${ }^{152} \mathrm{Sm}\left({ }^{16} \mathrm{O},{ }^{16} \mathrm{O}\right), \mathrm{E}=66,72 \mathrm{MeV}$; calculated $\sigma(\theta) .{ }^{152} \mathrm{Sm}\left({ }^{16} \mathrm{O}, \mathrm{X}\right), \mathrm{E}(\mathrm{cm}) \approx 55-70$ MeV; calculated fusion $\sigma(E)$. Dynamic polarjzation potential,coupled-channels effects.

$87 \times r 04$

\section{D.Krolle, K.Langanke - Z.Ph
MeV Resonant State in ${ }^{15} \mathrm{O}$}

NUCLEAR REACTIONS ${ }^{14} \mathrm{~N}(p, \gamma), E=0.5-3.5$ MeV; calculated radiative capture $\sigma(E)$. ${ }^{15} \mathrm{O} 1 \mathrm{evel}$ deduced $r$. Nuclear bremsstrahlung potential model.

$87 \mathrm{KrOg}$

S.Yu.Guskov, D.V.1lin, A.A.Levkovsky, V.B.Rozanov, V.E.Sherman - At.Energ. 63, 252 (1987): NUCLEAR REACTIUNS ${ }^{3} \mathrm{H}(\mathrm{d}, \mathrm{n}), \mathrm{E}=1 \mathrm{keV}-10 \mathrm{MeV} ;{ }^{3} \mathrm{He}(\mathrm{d}, \mathrm{p}), \mathrm{E}=0.001-1 \mathrm{MeV} ;$ calculated $\sigma(\mathrm{E})$. reaction rates.

K.H.Hahn, K.H.Chang, T.R.Donoghue, B.W.Filippone - Phys.Rev, C36, 892 (1987) - Search for NUCLEAR REACTIONS ${ }^{16} \mathrm{O}(\alpha, \gamma), \mathrm{E}(\mathrm{cm})=1.7-2.35 \mathrm{MeV}$; measured $\sigma(\mathrm{E}),\left({ }^{20} \mathrm{Ne}\right) \gamma$-coin, $\gamma$ yields. ${ }^{20} \mathrm{Ne}$ deduced resonances, $\Gamma$, resonance strength, nonresonant capture astrophysical $S$-factor.

NUCLEAR REACTIONS ${ }^{26} \mathrm{Mg}^{56} \mathrm{Fe}(\mathrm{p}, \mathrm{p}),\left(\mathrm{p}, \mathrm{p}^{\prime}\right), \mathrm{E}=8-14 \mathrm{MeV}$; analyzed $\sigma(\mathrm{E}, \theta),{ }^{28} \mathrm{Si}(\mathrm{d}, \alpha), \mathrm{E}=9 \mathrm{MeV}$; ${ }^{8 \theta} \mathrm{Zn}(\mathrm{p}, \mathrm{n}), \mathrm{E}=0.4-5.5 \mathrm{MeV} ;{ }^{5 \theta} \mathrm{Ni}(\alpha, p){ }^{56} \mathrm{Fe}(\mathrm{n}, \mathrm{p}), \mathrm{E}=4-30 \mathrm{MeV} ;$ anglyzed $\sigma(\mathrm{E}) .{ }_{1 \theta 1} \mathrm{Ta}(\mathrm{n}, \mathrm{p}), \mathrm{E} \leq 12$ $M e V,{ }^{40} \mathrm{Ca}(\mathrm{n}, \mathrm{X}), E=14.5 \mathrm{MeV}$; analyzed damping,escape widths. ${ }^{25} \mathrm{M}_{3}\left({ }^{3} \mathrm{He}, \alpha\right), E=8-20 \mathrm{MeV}$; analyzed five-excitation stage width. $\left.{ }^{27} \mathrm{~A}\right)\left({ }^{3} \mathrm{He}, \mathrm{p}\right){ }^{83} \mathrm{Nb},{ }^{181} \mathrm{Ta},{ }^{59} \mathrm{Co}(\mathrm{n}, \mathrm{X}), \mathrm{E}=2-20 \mathrm{MeV}$; analyzed $\sigma(E), \sigma(E n, \theta n) .{ }^{40} \mathrm{Ca}(n, p),{ }^{59} \mathrm{Co}(p, n), E=14.5 \mathrm{MeV}$; analyzed $\sigma(E p, \theta p), \sigma(E n, \theta n) .{ }^{59} \mathrm{Co}\left(n, n^{\prime}\right),(n$, $2 n),(n, p n),(n, p),(n, \alpha),(n, \alpha n), E=14 \mathrm{MeV}$; analyzed $\sigma$. Europhys.Lett. 4, 275 (1987) - Barriers and' Anomaly' of Symmetric to Asymmetric Yield Ratio NUCLEAR REACTIONS ${ }^{200} \mathrm{Bi}(\alpha, F), E=17-31 \mathrm{MeV}$; mea ured total,asymmetric fission $\sigma(E)$, rragment yield vs mass,energy distributions. ${ }^{213} \mathrm{At}$ deduced symmetric,asymmetric fission barrier heights.

M.G.Itkis, Yu.V.Kotlov, S.l.Mulgin, V.N.Okolovich, A.Ya.Rusanov, G.N.Smirenkin - Yad.Fiz. 46,

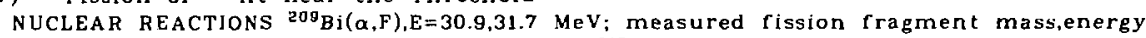
distributions; deduced fragment yield ratio. ${ }^{213} \mathrm{At}$ deduced fission barrier features.

A.Iwamoto, K.Harada - Z.Phys. A326, 201 (1987) - Enhancement or the Subbarrier Fusion Reaction NUCLEAR REACTIONS $4^{40} \mathrm{Ca}\left({ }^{\circ}{ }^{\circ} \mathrm{Ca}, \mathrm{X}\right), \mathrm{E}=45-65 \mathrm{MeV} ;{ }^{58} \mathrm{Ni}\left({ }^{58} \mathrm{Ni}, \mathrm{X}\right), \mathrm{E}=90-110 \mathrm{MeV} ;{ }^{84} \mathrm{Ni}\left({ }^{84} \mathrm{Ni}, \mathrm{X}\right)$, E5-110 MeV; ${ }^{74} \mathrm{Ge}\left({ }^{74} \mathrm{Ge}, X\right), E=105-135 \mathrm{MeV} ;{ }^{80} \mathrm{Se}\left({ }^{80} \mathrm{Se}, \mathrm{X}\right), \mathrm{E}=125-150 \mathrm{MeV} ;{ }^{90} \mathrm{Zr}\left({ }^{80} \mathrm{Zr}, \mathrm{X}\right)$, $E=170-195 \mathrm{MeV}$ i calculated fusion $\sigma(E)$, potential energies. Neck formation, Krappe-Nix-Sierk model.

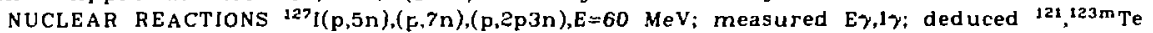
production relative yield to 1231 . Ge(Li) detector.

NUCLEAR REACTIONS ${ }^{28} \mathrm{Si}\left({ }^{12} \mathrm{C}, \mathrm{X}\right),{ }^{24} \mathrm{Mg}\left({ }^{16} \mathrm{O}, \mathrm{X}\right),{ }^{20} \mathrm{Ne}\left({ }^{20} \mathrm{Ne}, \mathrm{X}\right), \mathrm{E}(\mathrm{cm})=15-55 \mathrm{MeV}$; calculated rusion $\sigma(E)$. Direct reaction model.

A.Krauss, H.W.Becker, H.P.Trautvetter, C.Rolfs - Nucl.Phys. A467, 273 (1987) - Astrophysjcal $S(E)$ Factor of ${ }^{3} \mathrm{He}\left({ }^{3} \mathrm{He}, 2 \mathrm{p}\right)^{4} \mathrm{He}$ at Solar Energies 
NUCLEAR REACTIONS ${ }^{3} \mathrm{He}\left({ }^{3} \mathrm{He}, 2 \mathrm{p}\right), \mathrm{E}(\mathrm{cm})=17.9-342.5 \mathrm{keV}$; measured $\sigma(\mathrm{E}, \theta)$; deduced reaction $\sigma$. astrophysical factor $S(E)$. Windowless gas targets,beam calorimeter,cosmic background rejection,electronic shielding.

87Kr15 A.Krasznahorkay, T.Fenyes, J.Timar, T.Kibedi, A.Passoja, R.Julin, J.Kumpulainen - Nucl.Phys. A 473, 471 (1987) - Level Structure of ${ }^{110}$ In from the ${ }^{110} \mathrm{Cd}(p, n \gamma)^{110}$ ln Reaction

NUCLEAR REACTIONS ${ }^{110} \mathrm{Cd}(\mathrm{p}, \mathrm{n} \gamma), \mathrm{E}=5.2,5.6,6 \mathrm{MeV}$; measured $\mathrm{E} \gamma, \mathrm{l} \gamma, \gamma \gamma-\operatorname{coin}, \mathrm{l}(\mathrm{ce}), \mathrm{level} \sigma, \sigma(\mathrm{E}$. E $\gamma, \theta)$. ${ }^{110}$ In deduced levels, $\gamma$-branching, $\gamma$ mixing ratio,J, $\pi$. Ge(Li), hyperfine Ge detectors, combined magnetic plus $\mathrm{Si}(\mathrm{Li})$ electron spectrometer. Enriched targets.

B7Kr18 A.Krauss, H.w.Becker, H.P.Trautvetter, C.Rolfs, K.Brand - Nuci.Phys. A465, 150 (1987) Low-Energy Fusion Cross Sections of $D+D$ and $D+{ }^{J}$ He Reactions NUCLEAR REACTIONS ${ }^{2} \mathrm{H}(\mathrm{d}, \mathrm{n}),(\mathrm{d}, \mathrm{p}), \mathrm{E}(\mathrm{cm})=2.98-162.5 \mathrm{keV} ;{ }^{3} \mathrm{He}(\mathrm{d}, \mathrm{p}), \mathrm{E}(\mathrm{cm})=6.95-141.8 \mathrm{keV}$; measured $\sigma(E, \theta)$; deduced reaction $\sigma$,astrophysical $S(E)$ factor. Windowless gas targets. beam calorimeter.

$87 \mathrm{KrZV}$ R.M.Kremer - Diss.Abst.Int. 48B, 1725 (1987) - ${ }^{12} \mathrm{C}(\alpha, \gamma)^{10} \mathrm{O}$ Reaction at Low Energies NUCLEAR REACTIONS ${ }^{12} \mathrm{C}(\alpha, \gamma), E=1.29-3.29$; measured $\gamma \gamma(\mathrm{t})$; deduced absolute $\sigma(E), S-f a c t o r$.

87Ku02 A.Kuronen, J.Keinonen, P.Tikkanen - Phys.Rev. C35, 591 (1987) - Cross Section of ${ }^{180}+{ }^{180}$ near the Coulomb Barrier

NUCLEAR REACTIONS ${ }^{16} \mathrm{O}\left({ }^{16} \mathrm{O}, \mathrm{X}\right){ }^{2 \mathrm{~B}} \mathrm{Si} /{ }^{30} \mathrm{Si} /{ }^{30} \mathrm{P} /{ }^{31} \mathrm{P} /{ }^{31} \mathrm{~S} /{ }^{27} \mathrm{Al} /{ }^{24} \mathrm{Mg}, \mathrm{E}(\mathrm{cm})=8-13.8 \mathrm{MeV}$; measured E $\gamma, \mathrm{I} \gamma$, $\sigma(\theta), \mathcal{D S A}$; deduced residuals production $\sigma(E)$,astrophysical factors. Tantalum oxide target, Ge(Li) detector.

87La 04

S.Landowne, S.C.Pieper, F.Videbaek - Phys.Rev. C35, 597 (19R7) - Coupled-Channels Analysis or Silicon-Nickel Fusion Reactions

NUCLEAR REACTIONS ${ }^{38,62,64} \mathrm{Ni}\left({ }^{28} \mathrm{Si}, \mathrm{X}\right),\left({ }^{28} \mathrm{Si},{ }^{29} \mathrm{Si}\right),\left({ }^{30} \mathrm{Si}, \mathrm{X}\right),\left({ }^{28} \mathrm{Si},{ }^{27} \mathrm{Al}\right), \mathrm{E}(\mathrm{cm})=47-67 \mathrm{MeV}$ : calculated rusion,transfer reaction $\sigma(\theta), \sigma(E)$. Coupled-channels model.

87La08 M.C.Lagunas-Solar, O.F.Carvacho, L.Nagahara, A.Mishra, N.J.Parks - Appl.Radiat.isot. 38, 129 (198?) - Cyclotron Production of No-Carrier-Added ${ }^{206} \mathrm{Bi}(6.24 \mathrm{~d})$ and ${ }^{205^{\mathrm{Bi}}}(15.31 \mathrm{~d})$ as Tracers for Biological Studies and for the Development of Alpha-Emitting Radiotherapeutic Agents NUCLEAR REACTIONS $\mathrm{Pb}(\mathrm{p}, \mathrm{X})^{205} \mathrm{Bi} /{ }^{208} \mathrm{Bi}, \mathrm{E}=8.8-67.5 \mathrm{MeV}$; measured $\sigma(\mathrm{Ep})$, residual yields, total $\sigma$. Natural targets, Ge(Li), high-purity Ge detectors.

97La09 M.C.Lagunas-Solar, M.J.Avila, P.C.Johnson - Appl.Radiat.Isot. 38, 151 (1987) - Targetry and Radiochemical Methods for the Simultaneous Cyclotron Production of No-Carrier-Added Radiopharmaceutica1-Quality ${ }^{100} \mathrm{Pd},{ }^{97} \mathrm{Ru}$ and ${ }^{10: \mathrm{m}} \mathrm{Rh}$ NUCLEAR REACTIONS ${ }^{103} \mathrm{Rh}(\mathrm{p}, \mathrm{X}){ }^{100} \mathrm{Pd} /{ }^{87} \mathrm{Ru} /{ }^{101 \mathrm{~m}} \mathrm{Rh}, \mathrm{E}=23.3-67.1 \mathrm{MeV}$; measured residuals thick target yields vs E. Target dissolution,radiochernical method.

87La 1 F.L.Lang, C.w.Werntz, C.J.Crannell, J.I.Trombka, C.C.Chang - Phys.Rev. C35, 1214 (1987) - Cross Sections ror Production of the $15.10-\mathrm{MeV}$ and Other Astrophysically Significant Gamma-Ray Lines through Excitation and Spallation of ${ }^{12} \mathrm{C}$ and ${ }^{10} \mathrm{O}$ with Protons NUCLEAR REACTIONS ${ }^{12} \mathrm{C}_{1}{ }^{16} \mathrm{O}(\mathrm{p}, \gamma), \mathrm{E}=40,65,85 \mathrm{MeV}$; measured $\mathrm{E} \gamma, \mathrm{l} \gamma, \gamma$ production $\sigma(E)$. Other data input.

87La12 C.E.Laird, D.Flynn, R.L.Hershberger, F.Gabbard - Phys.Rev. C35, 1265 (1987) - Proton-90Zr Interaction at Sub-Coulomb Proton Energies

NUCLEAR REACTIONS ${ }^{90} \mathrm{Zr}(\mathrm{p}, \mathrm{p}), \mathrm{E}=2-7 \mathrm{MeV} ;{ }^{90} \mathrm{Zr}(\mathrm{p}, \mathrm{p} \gamma), \mathrm{E}=3.9-5.7 \mathrm{MeV} ;{ }^{80} \mathrm{Zr}(\mathrm{p}, \gamma), \mathrm{E}=1.9-5.7 \mathrm{MeV}$; measured $\sigma(\theta), \gamma$ yields; deduced radiative capture $\sigma(E)$, reaction $\sigma(E)$. ${ }^{2}$ No deduced $\gamma$ strents function.

B7Lo07 Long Xianguan, Peng Xiureng, He Fuqing - Chin.J.Nucl.Phys. 9, 48 (1987) - Excitation Functions for $\left.{ }^{187} \mathrm{Au}(\mathrm{d}, \mathrm{p})^{180} \mathrm{Au},{ }^{187} \mathrm{Au}(\mathrm{d}, 2 \mathrm{R})\right)^{187 \mathrm{~m}} \mathrm{Hg},{ }^{197} \mathrm{Au}(\mathrm{d}, 2 \mathrm{n})^{197} \mathrm{Hg}$ and ${ }^{197} \mathrm{Au}(\mathrm{d}, \mathrm{p} 2 \mathrm{n})^{186} \mathrm{Au}$ NUCLEAR REACTIONS ${ }^{197} \mathrm{~A} u(d, p),(d, 2 n),(d, 2 n p), E=6.6-13.1$ MeV; measured $\sigma(E)$. Activation method,hyperpure Ge detectors.

B7Lo12 G.Lo Bianco, P.Paruzzi, K.P.Schmittgen, R.Reinhardt, A.Gelberg, K.o.zell, P.von Brentano, N.Blasi - Nucl.Ptys. A470, 266 (1987) - Levels in ${ }^{138}$ Ce by In-Beam Spectroscopy

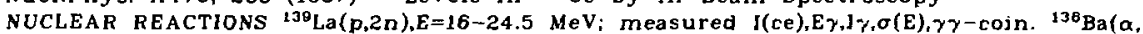

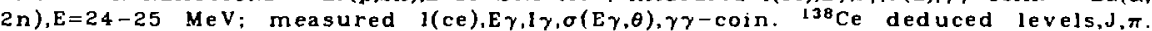
Mini-orange electron spectrometer,Ge( $\mathrm{Li})$, and Ge intrinsic detectors. Alaga model, $1 \mathrm{BM}-2$ calculations.

87 Ha50 M.T.Magda, A.Pop, A.Sandulescu - Rev.Roum.Phys. 32, 597 (1987) - Fragmentation Processes in Multinucleon Transfer Reactions 
NUCLEAR REACTIONS ${ }^{254} \mathrm{Es}\left({ }^{18} \mathrm{O}, \mathrm{X}\right){ }^{25 \mathrm{~B}} \mathrm{Lr} /{ }^{259} \mathrm{Lr} /{ }^{200} \mathrm{Lr} /{ }^{257} \mathrm{No} /{ }^{250} \mathrm{No} /{ }^{252} \mathrm{Fm} /{ }^{254} \mathrm{Fm} /{ }^{258} \mathrm{Fm} /{ }^{257} \mathrm{Fm} /{ }^{255} \mathrm{Md} /{ }^{250} \mathrm{Md} /$ ${ }^{257} \mathrm{Md} /{ }^{250} \mathrm{Md} /{ }^{259} \mathrm{Md} /{ }^{260} \mathrm{Md}, \mathrm{E}=98 \mathrm{MeV}:{ }^{254} \mathrm{Es}\left({ }^{22} \mathrm{Ne}, \mathrm{X}\right)^{252} \mathrm{Fm} /{ }^{254} \mathrm{Fm} /{ }^{255} \mathrm{Fm} /{ }^{256} \mathrm{Fm} /{ }^{257} \mathrm{Fm} /{ }^{254} \mathrm{Md} /{ }^{255} \mathrm{Md} /{ }^{256} \mathrm{Md} /$ ${ }^{257} \mathrm{Md} /{ }^{258} \mathrm{Md} /{ }^{250} \mathrm{Md} /{ }^{260} \mathrm{Md} /{ }^{254} \mathrm{No} /{ }^{255} \mathrm{No} /{ }^{256} \mathrm{No} /{ }^{257} \mathrm{No} /{ }^{259} \mathrm{No} /{ }^{256} \mathrm{Lr} /{ }^{258} \mathrm{Lr} /{ }^{250} \mathrm{Lr}, \mathrm{E}=126 \mathrm{MeV} ;{ }^{254} \mathrm{Es}\left({ }^{48} \mathrm{Ca}\right.$. $\mathrm{X})^{253} \mathrm{Fm} /{ }^{254} \mathrm{Fm} /{ }^{255} \mathrm{Fm} /{ }^{250} \mathrm{Fm} /{ }^{257} \mathrm{Fm} /{ }^{254} \mathrm{Md} /{ }^{255} \mathrm{Md} /{ }^{250} \mathrm{Md} /{ }^{257} \mathrm{Md} /{ }^{258} \mathrm{Md} /{ }^{258} \mathrm{No}, \mathrm{E}=266 \mathrm{MeV}$; calculated residuals production $\sigma$. Projectile fragmentation model.

87Mio5 P.Misaelides, J.Krauskopt, G.Wolf, K.Bethge - Nucl.Instrum.Methods Phys.Res. B18. 281 (1987) Determination of Carbon and Oxygen in GaAs by Means of ${ }^{3} \mathrm{He}$-Activation NUClEAR REACTIONS ${ }^{69} \mathrm{Ga},{ }^{73} \mathrm{As}\left({ }^{3} \mathrm{He}, \mathrm{n}\right),\left({ }^{3} \mathrm{He}, 2 \mathrm{n}\right),{ }^{80} \mathrm{Ga}\left({ }^{3} \mathrm{He}, a\right),\left({ }^{3} \mathrm{He}, \mathrm{na}\right),{ }^{71} \mathrm{Ca}\left({ }^{3} \mathrm{He}, 2 \mathrm{n}\right),\left({ }^{3} \mathrm{He}, 2 \mathrm{p}\right)$. ${ }^{10} \mathrm{Be}\left({ }^{3} \mathrm{He}, \mathrm{d}\right),\left({ }^{3} \mathrm{He}, \mathrm{np}\right), \mathrm{E}=2-12 \mathrm{MeV}$; measured $\sigma(\mathrm{E})$. Activation method.

87Mi07 F.Michel, G.Reidemeister, S.Ohkubo - Phys.Rev, C35, 1961 (1987) - Last Members of (he K( $\pi)=0,{ }^{+}$ $\alpha$-Cluster Rotational Band in ${ }^{20} \mathrm{Ne}$ NUCLEAR REACTIONS ${ }^{16} \mathrm{O}(a, y),(a, X), E=15-40 \mathrm{MeV}$; calculated reaction,fusion $\sigma(E)$.

87Mo27 Moon Hoe Cha, Jae Young Park, W.Scheid - Phys.Rev. C36, 234l (1987) - Molecular Single-Particle Effects in the ${ }^{12} \mathrm{C}+{ }^{17} 1^{18} \mathrm{O}$ and ${ }^{13} \mathrm{C}+{ }^{17} \mathrm{O}$ Reactions NUCLEAR REACTIONS ${ }^{17} \mathrm{O}\left({ }^{12} \mathrm{C}_{,}^{12} \mathrm{C}^{1}\right), \mathrm{E}(\mathrm{cm}) \approx 10-20 \mathrm{MeV} ;{ }^{10} \mathrm{O}\left({ }^{12} \mathrm{C},{ }^{12} \mathrm{C}\right), \mathrm{E}(\mathrm{cm}) \approx 22-32 \mathrm{MeV} ;{ }^{17} \mathrm{O}\left({ }^{13} \mathrm{C}\right.$. $\left.{ }^{13} \mathrm{C}^{\prime}\right), \mathrm{E}=10-20 \mathrm{MeV}$; calculated $\sigma(\mathrm{E})$. Two-center shell model,improved Landau-Zener model.

87 Mu08 M.G.Mustafa, T.Tamura, T.Udagawa - Phys.Rev. C35, 2077 (1987) - Direct-Reaction Plus

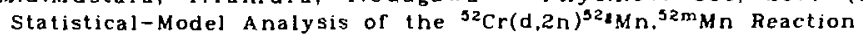

NUCLEAR REACTIONS ${ }^{52} \mathrm{Cr}(\mathrm{d}, 2 \mathrm{n}), E=8-20 \mathrm{MeV}$; calculated residual production $\sigma(E)$,isomer ratios. Direct reaction plus statistical model analyses.

87NgC5 Nguyen Kong Chang, V.A.Ageev, A.Kolachkovski, A.A.Klyuchnikov, V.A.Konov, N.A.Lebeder, A.F.Novgorodov, L.M.Popinenkova, M.Sobetska, V.A.Khalkin - Radiokhimiya 29, 241 (1987): Sov.J.Radiochemistry 29, 229 (1987) - Preparing ${ }^{167} \mathrm{Tm}$ from an Ytterbium Target Bombarded by 65-100 MeV Protons

NUCLEAR REACTIONS $Y b(p, x n)^{187} \mathrm{Tm}, E=65-100$ MeV: measured residual production yield following ${ }^{167} \mathrm{Lu}$ decay.

870h08 S.Ohkubo, D.M.Brink - Phys.Rev. C36, 966 (1987) - Internal and Barrier wave Interpretation of the Oscillations of the Fusion Excitation Function

NUCLEAR REACTIONS ${ }^{\circ 0} \mathrm{Ca}(\alpha, \alpha), E=5-20 \mathrm{MeV}$; calculated phase shifts vs $E$. ${ }^{40}, \mathbf{a}(\alpha, X), E=5-20$ MeV; calculated fusion $\sigma(E) .{ }^{44} \mathrm{Ti}$ deduced levels, $J, \pi$. Semi-classical model.

87Pal2 S.J.Padalino, K.Sartor, L.C.Dennis, K.W.Kemper - Phys.Rev. C35, 1692 (1987) - Direct and Fusion Carbon Reaction Components in the ${ }^{6} \mathrm{Li}+{ }^{12} \mathrm{C}$ Reaction at $30 \mathrm{MeV}$

NUCLEAR REACTIONS ${ }^{12} \mathrm{C}\left({ }^{6} \mathrm{Li}, a\right), \mathrm{E}=30 \mathrm{MeV}$; measured $\sigma(E a, \theta \alpha) .{ }^{6} \mathrm{Li}\left({ }^{12} \mathrm{C}, \mathrm{X}\right), \mathrm{E}=60 \mathrm{MeV}$; calculated rusion product yield vs charge distributions, ${ }^{12} \mathrm{C}$ spectrum, $\sigma(\theta) .{ }^{7} \mathrm{Li}\left({ }^{12} \mathrm{C}_{*}{ }^{12} \mathrm{C}\right), \mathrm{E}=58.4 \mathrm{MeV}$ : ${ }^{6} \mathrm{Li}\left({ }^{16} \mathrm{O},{ }^{16} \mathrm{O}\right), \mathrm{E}=68.6 \mathrm{MeV}$; calculated spectra. $\Delta \mathrm{E}-\mathrm{E}$ telescope. Statistical model.

87Pal9 E.Paleodimopoulos, T.Paradellis - J.Radioanal.Nucl.Chem. 117, 129 (1987) - Radiochemical Purification of Cadmium-109 Produced by the $109 / \mathrm{Ag} / \mathrm{p}, \mathrm{n} /$ Reaction NUCLEAR REACTIONS ${ }^{109} \mathrm{Ag}(\mathrm{p}, \mathrm{n}), \mathrm{E}=9 \mathrm{MeV}$; measured residual production yield. Radiochemical separation.

87 Pa24 G.Pantis, J.M.Pearson - !hys.Rev. C36, 1408 (1987) - Folding Model for Sub-Barrier lnteraction between Alpha-Type Nuclei

NUCLEAR REACTIONS ${ }^{18} \mathrm{O}\left({ }^{12} \mathrm{C},{ }^{12} \mathrm{C}\right), \mathrm{E}(\mathrm{cm}) \leq 12 \mathrm{MeV} ;{ }^{12} \mathrm{C}\left({ }^{12} \mathrm{C},{ }^{12} \mathrm{C}\right), \mathrm{E}(\mathrm{cm}) \leq 8 \mathrm{MeV} ;{ }^{16} \mathrm{O}\left({ }^{16} \mathrm{O},{ }^{16} \mathrm{O}\right), \mathrm{E}(\mathrm{cm})$ $514 \mathrm{MeV}$; calculated $\sigma\left(\theta=90^{\circ}\right)$, fusion $\sigma, S$-factor vs E. Folding model.

87Pi 08 J.Piekarewicz, S.E.Koonin - Phys.Rev. C36, 875 (1987) - Importance of the Deuteron Quadrupole Moment in ${ }^{2} \mathrm{H}(\mathrm{d}, y)^{4} \mathrm{He}$ NUCLEAR REACTIONS ${ }^{2} H(d, \gamma), E=0.075-3 \mathrm{MeV}$; calculated $\sigma(E)$; deduced ${ }^{2} H$ quadrupole component role. ${ }^{4}$ He deduced $D-$ to $S-s t a t e$ ratio. Phenomenological model.

87Pla3 R.Plaga, H.W.Becker, A.Redder, C.Rolfs, H.P.Trautvetter, K.Langanke - Nucl.Phys. A465. 291 (1987) - The Scattering of Alpha Particles from ${ }^{12} \mathrm{C}$ and the ${ }^{12} \mathrm{C}(\alpha, \gamma)^{26} \mathrm{O}$ stellar Reaction Rate NUCLEAR REACTIONS ${ }^{12} \mathrm{C}\left(\alpha_{,}, a_{0}\right), \mathrm{E}=1-66 \mathrm{MeV}$; measured $\sigma(E, \theta)$; deduced astrophysical $S$-ractor vs E.capture $\sigma .{ }^{18} \mathrm{O}$ deduced level parameters. Enriched ${ }^{12} \mathrm{C}$ targets.

87Poll V.Ponisch, S.E.Koonin - Phys.Rev. C36, 633 (1987) - Subbarrier Interactions or the Oxygen Isotopes

NUCLEAR REACTIONS $17,18 \mathrm{O}\left({ }^{10} \mathrm{O} . \mathrm{X}\right), \mathrm{E}(\mathrm{cm})=6-13 \mathrm{MeV}$; calculated fusion,total inelastic $\sigma(\mathrm{E})$. $\sigma(\theta)$,elastic to Rutherford ratios. Coupled-channels method. 
87Qa02 S.M.Qaim - Radiochim.Acta 41, 111 (1987) - Cyclotron Produclion of Generator Radionuclides NUCLEAR REACTIONS ${ }^{79} \mathrm{Br}(\alpha, 2 \mathrm{n}),{ }^{81} \mathrm{Br}\left({ }^{3} \mathrm{He}, 3 \mathrm{n}\right), \mathrm{E} \approx$ threshold-40 $\mathrm{MeV} ;{ }^{85} \mathrm{Rb}(\mathrm{p}, 4 \mathrm{np}), \mathrm{E} \approx 50-70 \mathrm{MeV}$; compiled reaction $\sigma(E)$. ${ }^{82} \mathrm{Kr}(\mathrm{p}, 2 \mathrm{n}), \mathrm{E} \approx \mathrm{threshold}-40 \mathrm{MeV}$; analyzed data; deduced $\sigma(E)$. Compiled cyclotron produced generator radionuclide data.

87Ra05 J.Rama Rao, A.V.Mohan Rao, S.Mukherjee, R.Upadhyay. N.L.Singh. S.Agarwal, L.Chaturvedi, P.P.Singh - J.Phys.(London) G13,535 (1987) - Non-Equilibrium Errects in Alpha-Particle-1nduced Reactions in Light,Medium and Heavy Nuclei up to $120 \mathrm{MeV}$

NUCLEAR REACTIONS ${ }^{51} \mathrm{~V},{ }^{83} \mathrm{Nb}(\alpha, n),{ }^{83} \mathrm{Nb},{ }^{165} \mathrm{Ho}(\alpha, 2 \mathrm{n}),{ }^{51} \mathrm{~V}(\alpha, 3 \mathrm{n}),{ }^{105} \mathrm{Ho}(\alpha, 4 \mathrm{n}), \mathrm{E}=10-120$ MeV; measured $\sigma(E)$. Activation method. Pre-equilibrium hybrid model.

87Ra0B J.Rama Rao, A.V.Mohan Rao, S.Mukherjee, R.Upadhyay, N.L.Singh, S.Agarwal, L.Chaturvedi, P.P.Singh - Nucl.Instrum.Methods Phys.Res. B24/25, 484 (1987) - Excitation Functions of Alpha Particle Induced Reactions in Cobalt in the Energy Range 10-120 MeV using Variable Energy Cyclotrons

NUCLEAR REACTIONS ${ }^{50} \mathrm{Co}(\alpha, 5 \mathrm{np}),(\alpha 6 \mathrm{np}),(\alpha, n 2 \mathrm{p}),(\alpha, n 3 \mathrm{p}),(\alpha, n \alpha),(\alpha, 2 \mathrm{n} \alpha),(\alpha, 3 \mathrm{n} \alpha),(\alpha, n 2 \alpha),(\alpha$, $3 n 2 \alpha),(\alpha, 3 n p 2 \alpha), E=10-120 \mathrm{MeV}$; measured residue production $\sigma(E)$. Activation method, Ge(Li) detector.

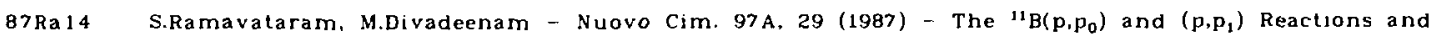
the Continuum Model of Nuclear Reactions

NUCLEAR REACTIONS "B(p,p),(p,p'),E=19.6-25 MeV; calculated $\sigma(E)$,analyzing powers vs E. ${ }^{12} \mathrm{C}$ deduced resonance characteristics. Coupled-Channels model.

$87 \operatorname{Ra} 23$

J.Raisanen, T.Witting, J.Keinonen - Nucl.lnstrum.Methods Phys.Res. B28, 199 (1987) - Absolute Thick-Target $\gamma$-Ray Yields for Elemental Analysis by 7 and 9 MeV Protons NUCLEAR REACTIONS ${ }^{7} \mathrm{Li},{ }^{9} \mathrm{Be},{ }^{10},{ }^{11} \mathrm{~B},{ }^{14} \mathrm{~N},{ }^{16} \mathrm{O},{ }^{19} \mathrm{~F},{ }^{23} \mathrm{Na},{ }^{24},{ }^{26} \mathrm{Mg},{ }^{27} \mathrm{Al},{ }^{28},{ }^{29},{ }^{30} \mathrm{Si},{ }^{31} \mathrm{P},{ }^{32} \mathrm{~S},{ }^{35} \mathrm{Cl},{ }^{39} \mathrm{~K},{ }^{40} \mathrm{Ca},{ }^{46}$. ${ }^{47},{ }^{48} \mathrm{Ti},{ }^{52} \mathrm{Cr},{ }^{55} \mathrm{Mn},{ }^{54}, 56 \mathrm{Fe},{ }^{59} \mathrm{Co},{ }^{58},{ }^{60} \mathrm{Ni},{ }^{53} \mathrm{Cu},{ }^{64} \mathrm{Zn},{ }^{70},{ }^{72} \mathrm{Ge},{ }^{89} \mathrm{Y}^{900} \mathrm{Zr},{ }^{93} \mathrm{Nb},{ }^{92},{ }^{95} \mathrm{Mo},{ }^{90},{ }^{104} \mathrm{Ru},{ }^{107},{ }^{09} \mathrm{Ag},{ }^{11 !}$,

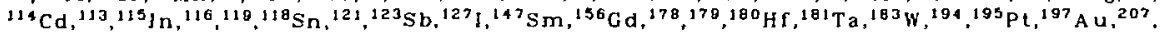
${ }^{200} \mathrm{~Pb}(\mathrm{p}, \gamma),(\mathrm{p}, \mathrm{n}), \mathrm{E}=7-9 \mathrm{MeV}$; measured absolute thick target $\gamma$ yieldirelative neutron yield. $G e(L i), B F_{3}$ detectors.

87Ra26

C.Rangacharyulu, G.Kuechler, A.Richter, E.Spamer - Astrophys.J. 320, 405 (1967) - Radiative Transitions in ${ }^{27} \mathrm{Al}$ and Thejr Rejevance to the ${ }^{26} \mathrm{Al}(\mathrm{p}, y)^{27} \mathrm{Si}$ Reaction Under Stellar Conditions NUCLEAR REACTIONS ${ }^{27} \mathrm{Al}\left(\mathrm{e}, \mathrm{e}^{\prime}\right) . \mathrm{E}=25,35,40 \mathrm{MeV}$; measured electron scattering spectra. ${ }^{27} \mathrm{Al}$ deduced $\gamma$-transition strength.s. ${ }^{26} \mathrm{Al}(\mathrm{p}, \gamma), \mathrm{E}=0.05-0.15 \mathrm{MeV}$; calculated reaction rale, .

87 Re02

A.Redder, H.W.Becker, C.Rolfs, H.P.Trautvetter, T.R.Donoghue. T.C.Rinckel, J.w.Hammer. K.Langanke - Nucl.Phys. A 462, $385(1987)-$ The ${ }^{12} \mathrm{C}(\alpha, \gamma)^{16} \mathrm{O}$ Cross Section at Stellar Energies NUCLEAR REACTIONS ${ }^{12} \mathrm{C}(\alpha, \gamma), \mathrm{E}=0.94-2.84$ MeV; measured $\mathrm{E} \gamma, \mathrm{l} \gamma, \sigma(\mathrm{E}), \sigma(\mathrm{E}, \mathrm{E}(\gamma), \theta)$; deduced astrophysical $S-$ ractor. Implanted ${ }^{12} \mathrm{C}$ targets.NaI(Tl),Ge detectors.

87Ri09

I.A.Rizyi, M.Afzal Ansari. R.P.Gautam, R.K.Y.Singh, A.K.Chaubey - J.Phys.Soc.Jpn. 56,3135 (1987) - Excitation Function Studies of $(\alpha, x p y n)$ Reactions for $63.65 \mathrm{Cu}$ and Pre Equilibrium Effect NUCLEAR REACTIONS ${ }^{83,65} \mathrm{Cu}(\alpha, x \cap y \mathrm{p})^{66} \mathrm{Ga} /{ }^{85} \mathrm{Zn} /{ }^{65} \mathrm{Ga} /{ }^{68} \mathrm{Ga} /{ }^{67} \mathrm{Ga}, \mathrm{E}=40 \mathrm{MeV}$; measured residual production $\sigma(E)$. Stacked foll activation technique. Preequilibrium emission,geometry dependent hybrid model.

B7RoO E.A.Romanivsky, T.l.Spasskaya, A.M.Borisov. S.V.Ermakov, Nguen Mak Kha. B.M.Saidov, N.G.Goryaga, B.S.Galakhmatova, S.A.Goncharov, Khussein Khamad Abbd - Izv.Akad.Nauk SSSR, Ser.Fiz. 51, 24 (I987) - Optical Potential for Description of Proton Scattering on ${ }^{38}$ Si, ${ }^{32} \mathrm{~S}$ Nuciei at Intermediate and Low Energies NUCLEAR REACTIONS ${ }^{28} \mathrm{Si}^{32} \mathrm{~S}(\mathrm{p}, \mathrm{p}), E=40 \mathrm{MeV}$; calculated $\sigma\left(E_{p}, \theta p\right)$, reaction $\sigma(E)$; deduced optical potential parameters.

87Sa49 S.K.Saha, R.Guin, S.M.Sahakundu - J.Radioanal.Nucl.Chem. 119, 303 (1987) - 1somer Ratios of 80. ${ }^{87} \mathrm{Y}$ and ${ }^{84} \mathrm{Rb}$ in the Alpha induced Reaction on ${ }^{85} \mathrm{Rb}$

NUCLEAR REACTIONS ${ }^{85} \mathrm{Rb}(\alpha, 2 \mathrm{n}),(\alpha, 3 \mathrm{n}),(\alpha, n \alpha), \mathrm{E}=20-60 \mathrm{MeV}$; measured residuals isorner $y$ ield ratios VS E.

87Sa52 S.J.Sanders, D.G.Kovar, B.B.Back, C.Beck, B.K.Dichter, D.Henderson, R.V.F.Janssens, J.C.Keller, S.Kaufman. T.-F.Wang, B.Wilkins, F.Videbaek - Phys.Rev.Lett. 59, 2856 (1987) - Asymmetric Fission of ${ }^{38} \mathrm{Ni}$

NUCLEAR REACTIONS ${ }^{24} \mathrm{Mg}\left({ }^{32} \mathrm{~S}, \mathrm{X}\right), \mathrm{E}=141.8 \mathrm{MeV}$; measured mass, velocity spectra,binary reaction $\sigma(A)$ i deduced fission reaction yield characteristics. "No ${ }^{\prime}$ 
$87 S c 10$ U.Schroder, H.W.Becker, G.Bogaert, J.Gorres, C.Rolfs, H.P.Trautvetter, R.E.Azuma, C.Campbell, J.D.King, J.Vise - Nucl.Phys. A467. 240 (1987) - Stellar Reaction Rate of ${ }^{14} N(p, \gamma)^{15} 0$ and Hydrogen Burning in Massive Stars

NUCLEAR REACTIONS ${ }^{14} \mathrm{~N}(p, \gamma), E=0.2-3.6 \mathrm{MeV}$; measured $\sigma(\mathrm{E}, \mathrm{E}(\gamma), \theta(\gamma))$; deduced astrophysica) $S$-factor,capture $\sigma$. ${ }^{15} \mathrm{O}$ deduced levels, $J, \pi, \Gamma$, spectroscopic factors. Windowless gas, implanted ${ }^{14} \mathrm{~N}$ targets. $\mathrm{Ge}(\mathrm{Li})$ detectors

87Sc18 U.Schruder, A.Redder, C.Rolfs, R.E.Azuma, L.Buchmann, C.Campbell, J.D.King. T.R.Donoghue Phys.Lett. 142B, 55 (1987) - Astrophysical S Factor of ${ }^{3} \mathrm{H}(a, \gamma)^{7} \mathrm{Li}$

NUCLEAR REACTIONS ${ }^{3} \mathrm{H}(a, \gamma), \mathrm{E}(\mathrm{cm})=79-464 \mathrm{keV}$; measured E $\gamma$, I $\gamma$, branching ratio, $\sigma(E) . \gamma$ yield: deduced astrophysical S-factor vs E. Ge(Li) detectors.

87 Sc27 A.F.Scott, C.1.W.Tingwell, V.Y.Hansper, S.G.Tims, D.G.Sargood - Nucl.Phys. A475, 548 (19B7) The ${ }^{39} \mathrm{~K}(\alpha, p)^{+2} \mathrm{Ca}$ Cross Section and Thermonuclear Reaction Rate NUCLEAR REACTIONS ${ }^{39} \mathrm{~K}(\alpha, p), E=4.5-9 \mathrm{MeV}$; measured proton yield; deduced $\sigma(E)$,thermonuclear reaction rates. Statistical-model analysis. Enriched targets.

B7Sc33 A.Scalia - Nuovo Cim. 98A, 571 (1987) - Subbarrier Fusion with the Elastic Model

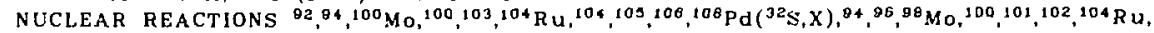
${ }^{103} \mathrm{Rh}^{104},{ }^{105},{ }^{106}, 108,{ }^{10} \mathrm{Pd}\left({ }^{30} \mathrm{~S}, \mathrm{X}\right), \mathrm{E}(\mathrm{cm}) \approx 76-92 \mathrm{MeV} ;{ }^{82},{ }^{80},{ }^{96} \mathrm{Mo}\left({ }^{32} \mathrm{~S}, \mathrm{X}\right),{ }^{100} \mathrm{Mo}\left({ }^{38} \mathrm{~S}, \mathrm{X}\right), \mathrm{E}(\mathrm{cm}) \approx$ $74-90 \mathrm{MeV} ;{ }^{100} \mathrm{Mo}\left({ }^{32} \mathrm{~S}, \mathrm{X}\right), \mathrm{E}(\mathrm{cm}) \approx 72-88 \mathrm{MeV} ;{ }^{101} \mathrm{Ru}\left({ }^{32} \mathrm{~S}, \mathrm{X}\right) . \mathrm{E}(\mathrm{cm}) \approx 80-92 \mathrm{MeV} ;{ }^{108} \mathrm{Pd}\left({ }^{32} \mathrm{~S}, \mathrm{X}\right)$, $\mathrm{E}(\mathrm{cm}) \approx 78-94 \mathrm{MeV} ;{ }^{14} \mathrm{Sm}\left({ }^{40} \mathrm{Ar}, \mathrm{X}\right), \mathrm{E}(\mathrm{cm}) \approx 114-146 \mathrm{MeV} ;{ }^{154} \mathrm{Sm}\left({ }^{40} \mathrm{Ar}, \mathrm{X}\right), \mathrm{E}(\mathrm{cm}) \approx 112-144 \mathrm{MeV}:{ }^{150}$, ${ }^{152}, 154,1^{48} \mathrm{Sm}\left({ }^{16} \mathrm{O}, \mathrm{X}\right), \mathrm{E}(\mathrm{cm}) \approx 52-68 \mathrm{MeV} ;{ }^{144} \mathrm{Sm}\left({ }^{40} \mathrm{Ar}, \mathrm{X}\right), \mathrm{E}(\mathrm{cm}) \approx 120-140 \mathrm{MeV} ;{ }^{27} \mathrm{Al}\left({ }^{35} \mathrm{Cl}, \mathrm{X}\right), \mathrm{E}(\mathrm{cm})$ $\approx 30-70 \mathrm{MeV} ;{ }^{62} \mathrm{Ni}\left({ }^{25} \mathrm{Cl}, \mathrm{Y}\right), \mathrm{E}(\mathrm{cm}) \approx 58-110 \mathrm{MeV} ;{ }^{148} \mathrm{Nd}\left({ }^{18} \mathrm{O}, \mathrm{X}\right), \mathrm{E}(\mathrm{cm}) \approx 54-70 \mathrm{MeV} ;{ }^{148,150} \mathrm{Nd}\left({ }^{16 \mathrm{O}}\right.$, $\mathrm{X}, \mathrm{E}(\mathrm{cm}) \approx 56-72 \mathrm{MeV} ;{ }^{150} \mathrm{Nd}\left({ }^{16} \mathrm{O}, \mathrm{X}\right), \mathrm{E}(\mathrm{cm}) \approx 14-26 \mathrm{MeV} ;{ }^{150} \mathrm{~Tb}\left({ }^{7} \mathrm{Li}, \mathrm{X}\right),{ }^{44} \mathrm{Ca}\left({ }^{18} \mathrm{O}, \mathrm{X}\right), \mathrm{E}(\mathrm{cm}) \approx 18-30$ $\mathrm{MeV} ;{ }^{152} \mathrm{Sm}\left({ }^{12} \mathrm{C}, \mathrm{X}\right), \mathrm{E}(\mathrm{cm}) \approx 42-58 \mathrm{MeV} ;{ }^{38} \mathrm{Ni}\left({ }^{35} \mathrm{Cl}, \mathrm{X}\right), \mathrm{E}(\mathrm{cm}) \approx 60-92 \mathrm{MeV} ;{ }^{44} \mathrm{Ca}\left({ }^{40} \mathrm{Ca}, \mathrm{X}\right), \mathrm{E}(\mathrm{cm}) \approx$ 48-54 MeV; ${ }^{58} \mathrm{Ni}\left({ }^{58} \mathrm{Ni}, \mathrm{X}\right), \mathrm{E}(\mathrm{cm}) \approx 92-108 \mathrm{MeV} ;{ }^{12} \mathrm{C}\left({ }^{12} \mathrm{C}, \mathrm{X}\right), \mathrm{E}(\mathrm{cm}) \approx 3-5 \mathrm{MeV} ;{ }^{16} \mathrm{O}\left({ }^{12} \mathrm{C}, \mathrm{X}\right), \mathrm{E}(\mathrm{cm}) \approx$ 5-13 $\mathrm{MeV} ;{ }^{20} \mathrm{Ne}\left({ }^{12} \mathrm{C}, \mathrm{X}\right), \mathrm{E}(\mathrm{cm}) \approx 8-15 \mathrm{MeV} ;{ }^{10} \mathrm{O}\left({ }^{16} \mathrm{O}, \mathrm{X}\right), \mathrm{E}(\mathrm{cm}) \approx 7-12 \mathrm{MeV}$; calculated rusion $\sigma(E)$.

87Se13 S.Seuthe, H.W.Becker, A.Krauss, A.Redder, C.Rolfs, U.Schroder, H.P.Trautvetter, K.Wolke, S.Wustenbecker, R.W.Kavanagh, F.B.Waanders - Nucl.Instrum.Methods Phys.Res. A260, 33 (1987) Production and Properties of Implanted Targets NUCLEAR REACTIONS ${ }^{12} \mathrm{C}(\mathrm{p}, \gamma), \mathrm{E}=220-340 \mathrm{keV} ;{ }^{12} \mathrm{C}(a, \gamma), \mathrm{E}=2.65-2.85 \mathrm{MeV} ;{ }^{14} \mathrm{~N}(\mathrm{p}, \gamma), \mathrm{E}=260-360$ $\mathrm{keV} ;{ }^{20} \mathrm{Ne}(\mathrm{p}, \gamma), \mathrm{E}=1165-1185 \mathrm{keV} ;{ }^{23} \mathrm{Na}(\mathrm{p}, \gamma), \mathrm{E}=300-400 \mathrm{keV}$; measured $\gamma$-ray yields. Implanted targets.

$875 h 06$

B.Shivakumar, S.Ayik, B.A.Harmon, D.Shapira - Phys, Pev. C35, 1730 (1987) - Equilibrium Model for Fusion and Orbiting

NUCLEAR REACTIONS ${ }^{20} \mathrm{Si}\left({ }^{12} \mathrm{C}, \mathrm{X}\right), \mathrm{E}(\mathrm{cm})=25-60 \mathrm{MeV}$; calculated fusion,orbiting $\sigma(E)$, fragment kinetic energies. Equilibrium model.

87Sh30 K.Shikano, M.Katoh, T.Shigematsu, H.Yonezawa - J.Radioanal.Nucl.Chem. 119, 433 (1987) Production of the Radioisutope ${ }^{8 B} \mathrm{Y}$

NUCLEAR REACTIONS ${ }^{88} \mathrm{Sr}(p, n), E=16 \mathrm{MeV} ;{ }^{87} \mathrm{Sr}(d, n), E=7.3 \mathrm{MeV} ;{ }^{87} \mathrm{Rb}\left({ }^{3} \mathrm{He}, 2 n\right), E=19.3 \mathrm{MeV} ;{ }^{85} \mathrm{Rb}(\alpha$, n), E = 12.8 MeV; measured thick target residual $y$ ield.

a7si11 N.L.Singh, S.Agerwal, L.Chaturvedi, J.Rama Rao - Nucl.Instrum.Methods Phys.Res. B24/25, 480 (1987) - Excitation Functions for Radioactive Isotopes Produced by Alpha Particle Induced Reactions with Silver

NUCLEAR REACTIONS ${ }^{107} \mathrm{Ag}(\alpha, n),(\alpha, 2 n),(a, n \alpha),(\alpha, 2 n a),{ }^{109} \mathrm{Ag}(a, 2 n),(\alpha, 3 n),(a, 4 n), \mathrm{E} \leq 50 \mathrm{MeV}$; measured residual production $\sigma(E)$. Activation method,Ge(Lj) detector.

87Sk03 E.A.Skakun, V.G.Baty, Yu.N.Rakivnenko, O.A.Rastrepin - Yad.Fiz. 45, 614 (1987) - Cross Sections of $(p, \gamma)$ Reactions on Isotopes ${ }^{54} \mathrm{Fe},{ }^{112} \mathrm{Sn}$ and ${ }^{114} \mathrm{Cd}$ at Proton Energies up to $9 \mathrm{MeV}$ NUCLEAR REACTIONS ${ }^{34} \mathrm{Fe},{ }^{12} \mathrm{Sn}, 14 \mathrm{Cd}(\mathrm{p}, \gamma), \mathrm{E}=3-9 \mathrm{MeV}$; measured $\sigma(\mathrm{E})$. Ge(Li) detector. Statistical theory.

87Sk06 E.A.Skakun, V.G.Batij. Yu.N.Rakivnenko, O.A.Rastrepin - Yad.Fiz. 46, 28 (1987) - Excitation Functions and 1somer Ratios for up-to-9-MeV Proton interactions with $\mathrm{Zr}$ and Mo Isotope Nuclei NUCLEAR REACTIONS ${ }^{80},{ }^{82},{ }^{8 \theta} \mathrm{Zr},{ }^{84},{ }^{85},{ }^{86},{ }^{100} \mathrm{Mo}(\mathrm{p}, \mathrm{n}), \mathrm{E} \leq 9 \mathrm{MeV}$; measured $\sigma(\mathrm{E}) .{ }^{82} \mathrm{Mo}(\mathrm{p}, \gamma), \mathrm{E} \leq 9$ $\mathrm{MeV}$; measured isomer production $\sigma(E)$,ratios. Hauser-Feshbach calculations.

87Sk07 S.J.Skorka, A.M.Stefanini, G.Fortuna, R.Pengo, W.Meczynski, G.Montagnoli, A.Tivelli, S.Beghini, C.Signorini, P.R.Pascholati - 2.Phys. A328, 355 (1987) - Sub-Barrier Fussion of ${ }^{35},{ }^{37} \mathrm{Cl}+58,{ }^{84} \mathrm{Ni}$ Evidence of Effects of Proton Trangfer Channels NUCLEAR REACTIONS ${ }^{58,64} \mathrm{Ni}\left({ }^{35} \mathrm{Cl}, \mathrm{X}\right),\left({ }^{37} \mathrm{Cl}, \mathrm{X}\right), \mathrm{E} \approx 57-70 \mathrm{MeV}$; measured fusion $\sigma(\mathrm{E})$. Enriched targets. 
87Spos

87Sp11

87St 06

875403

87 Ta09

87Ta 14 O. Tanim
Section

NUCLEAR REACTIONS ${ }^{148,130,154} \mathrm{Sm}\left({ }^{16} \mathrm{O}, \mathrm{X}\right), \mathrm{E}=57-76 \mathrm{MeV} ;{ }^{232} \mathrm{Th}\left({ }^{16} \mathrm{O}, \mathrm{X}\right), \mathrm{E}=78-107 \mathrm{MeV} ;{ }^{236} \mathrm{U}\left({ }^{12} \mathrm{C}, \mathrm{X}\right)$, $E=58-85 \mathrm{MeV}$; calculated $\sigma(E)$. Coupled-channels approach.

87Tu01 A.Turowiecki, A.Saganek, M.Sieminski, E.Wesolowski, Z.Wilhelmi - Nucl.Phys. A468, 29 (1987) The ${ }^{11} \mathrm{~B}(a, p)^{14} \mathrm{C}$ Reaction below 1.7 MeV bombarding Energy NUCLEAR REACTIONS ${ }^{11} \mathrm{~B}(a, p), E=0.9-1.7 \mathrm{MeV}$; measured residual production $\sigma(E)$. ${ }^{25} \mathrm{~N}$ deduced resonances,partial widths.

87 Va 36

V.S.Vasilevsky, I.F.Gutjch, I.P.Okhrimenko - Yad.Fiz. 46, 757 (1987); Sov.J.Nucl.Phys. 46, 427 (1987) - Calculation of the Cross Section for the $d(t, n) a$ Reaction and of the Parameters of the $(3 / 2)^{*}$ Resonance of ${ }^{5} \mathrm{He}$ NUCLEAR REACTIONS ${ }^{2} H(t, n), E=0-300$ keV; calculated $\sigma(E)$, astrophysical S-factor vs E. ${ }^{2} H(t$, $t), E=0-3 \mathrm{MeV}$; $\mathrm{He}(\mathrm{n}, \mathrm{n}), \mathrm{E}$ not given; calculated phase shifts. ${ }^{5} \mathrm{He}$ deduced resonance parameters. Resonating group method.

87Vi01 K.Vjerinen - Nucl.Phys. A463, 605 (1987) - High Resolutjon Gamow-Teller Strength Measurement via $\beta$-Decay of ${ }^{65} \mathrm{Ge}$

NUCLEAR REACTIONS ${ }^{64} \mathrm{Zn}(\mathrm{p}, \gamma), \mathrm{E}=1.1-2.1 \mathrm{MeV}$; measured gamma yield, $\sigma(\mathrm{E}), \mathrm{E} \gamma, \mathrm{I} \gamma$. ${ }^{65} \mathrm{Ga}$ deduced resonance decays,proton binding energy. Enriched target,Ge(Li),NaI(TI) detectors.

87 Vu02

V.A.Vukolov, F.E.Chukreev - At.Energ. 62, 232 (1987); Sov.At.Energy 62, 271 (1987) - Neutron Yield for Chemical Compounds of Actinides

NUCLEAR REACTIONS Li, ${ }^{9} \mathrm{Be}, \mathrm{B}, \mathrm{C},{ }^{19} \mathrm{~F}(\alpha, n), \mathrm{E} \leq 10 \mathrm{MeV}$; compiled $\sigma(\mathrm{E})$, neutron yields. Actinide compounds.

87 We05

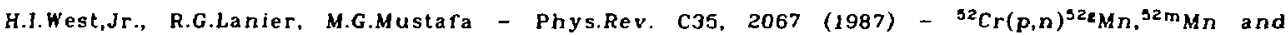
${ }^{52} \mathrm{Cr}(\mathrm{d}, 2 n)^{52 \mathrm{Mn}},{ }^{32 \mathrm{~m}} \mathrm{Mn}$ Excitation Functions

NUCLEAR REACTIONS $\mathrm{Cr}(\mathrm{p}, \mathrm{X})^{52} \mathrm{Mn} /{ }^{52 \mathrm{~m}} \mathrm{Mn},{ }^{52} \mathrm{Cr}(\mathrm{p}, \mathrm{n}), \mathrm{E}=6-27 \mathrm{MeV} ;{ }^{52} \mathrm{Cr}(\mathrm{d}, 2 \mathrm{n}), \mathrm{E}=8-20 \mathrm{MeV}$; measured residual production $\sigma(E), i s o m e r$ ratios. Activation method. Statistical model calculations.

87Wil1 M.Wiescher, V.Harms, J.Gorres, F.-K.Theilemann, L.J.Rybarcyk - Astrophys.J. 316, J62 (1987) Alpha-Burning of ${ }^{14} \mathrm{O}$

NUCLEAR REACTIONS ${ }^{14} \mathrm{O}(\alpha, p),(\alpha, \gamma), \mathrm{E}$ not given; calculated capture reaction,depletion rate ratios,S-factor, reaction $\sigma$. ${ }^{4} 0$ deduced $a$-burning characteristics.

$87 \mathrm{ZaO} 09$

N.G.Zaitgeva, A.S.Kovalev, O.Knotek, V.A.Khalkin, V.A.Ageev, A.A.Klyuchnikov, A.F.Linev Radiokhimiya 29, 247 (1987); Sov.J.Radiochemistry 29, 235 (1987) - Preparing 201 TI from Lead Bombarded by Protons of Energy about $65 \mathrm{MeV}$ 
NUCLEAR REACTIONS $\mathrm{Pb}(\mathrm{p}, \mathrm{xn})^{201} \mathrm{Tl} /{ }^{202} \mathrm{Tl}, \mathrm{E}=65 \mathrm{MeV}$ : measured residual yields.

87Za11 N.G.Zaitseva, O.Knotek, Kim Sen Han, P.Mikets, V.l.Sobolev, V.A.Khalkin, v.A.Konov, L.M.Popinenkova - Radiokhimiya 29, 391 (1987); Sov.J.Radiochemistry 29, 376 (1987) - Obtaining lodine-123 by Bombarding Sodium lodide with 100-MeV Protons NUCLEAR REACTIONS ${ }^{127} \mathrm{I}(\mathrm{p}, 3 \mathrm{n}),(\mathrm{p}, 5 \mathrm{n}), \mathrm{E}=100 \mathrm{MeV}$; measured residual production $\sigma$. Nal target.

87Zio2 F.Zijderhand, R.C.Makkus, C.van der Leun - Nucl.Phys. A466, 280 (1987) - Investigation of 4'sc by Proton Capture in ${ }^{40} \mathrm{Ca}$

NUCLEAR REACTIONS ${ }^{40} \mathrm{Ca}(p, \gamma), E=640-3500 \mathrm{keV}$; measured $\sigma(E(p)), \sigma(E(\gamma), \theta), \gamma$ yield: deduced Q. ${ }^{41} \mathrm{Sc}$ deduced levels, $\gamma$-branchings,J, $\pi, \delta, 1 \gamma,[\cdot \mathrm{p}, \Gamma$. Compton-suppression spectrometer. natural target. Shell-model calculations.

BrZw01 J.Zweit, H.Sharma, S.Downey - Appl.Radiat.lsot. 38, 499 (1987) - Production of Gallium-66,a Short-lived,Positron Emitting Radionuclide NUCLEAR REACTIONS $\mathrm{Cu}(\alpha, \mathrm{X})^{66} \mathrm{Ga} /{ }^{67} \mathrm{Ga} /{ }^{86} \mathrm{Cu} /{ }^{65} \mathrm{Zn}, \mathrm{E} \leq 40 \mathrm{MeV}$ i measured residues $\sigma(E)$. Natural target,Ge(Li) detector.

88A124

T.Altmeyer, E.Kolbe, T.Warmann, K.Langanke, H.J.Assenbaum - Z.Phys. A330, 277 (1988) - On Discrepancies within the Experimental and Theoretical Low Energy ${ }^{3} \mathrm{H}(\alpha, \gamma)^{7} \mathrm{Li}$ Cross Sections NUCLEAR REACTIONS ${ }^{3} H(a, \gamma), E \approx 50-600 \mathrm{keV}$; calculated $\sigma(E)$, -factor vs E. Resonating group method.

88An 11 J.D.Anderson, R.W.Bauer, V.R.Brown, S.M.Grimes, V.A.Madsen, B.A.Pohl, C.H.Poppe, W.Scobel Phys.Rev. C38, 1601 (1988) - Analog (p,n) Cross Sections of the Zirconium Isotopes at 18 and 25 $\mathrm{MeV}$

NUCLEAR REACTIONS $90,91,92,94 \mathrm{Zr}(p, n), E=18,25$ MeV; measured $\sigma(\theta)$; deduced reaction mechanism. ${ }^{90}, 919 ?{ }^{93} \mathrm{Nb}$ deduced analog excitation $\sigma .{ }^{91} \mathrm{Zr}(\mathrm{p}, \mathrm{n}), \mathrm{E} \approx 12-40 \mathrm{MeV}$; analyzed $\sigma(E)$.

88Aro9 S.E.Arnell, S.Mattsson, H.A.Rr,th, M.Rydehell, O.Skeppstedt, J.Nyberg, A.Johnson, A.Kerek, A.Nilsson, L.Westerberg - E.Phys. A330, 69 (1988) - Non-Equilibrium Neutron Emission in a-Induced Reactions on ${ }^{124} \mathrm{Sn}$

NUCLEAR REACTIONS ${ }^{124} \operatorname{Sn}(\alpha, 2 n),(\alpha, 3 n),(\alpha, 4 n),(\alpha, 5 n), E=35-57$ MeV; measured $\sigma(E)$, $\gamma$-multiplicities vs $E, \gamma n-$ coin.

88Au03 F.Auger, B.Fernandez -- Nucl.Phys. A481, 577 (1988) - Search for Low-L Fusion Window in the ${ }^{16} \mathrm{O}+$ ${ }^{16} \mathrm{O}$ Reaction at $4.5 \mathrm{MeV} / \mathrm{Nucleon}$

NUCLEAR REACTIONS ${ }^{16} \mathrm{O}\left({ }^{18} \mathrm{O}, X\right),{ }^{16} \mathrm{O}\left({ }^{16} \mathrm{O},{ }^{22} \mathrm{C}\right),\left({ }^{10} \mathrm{O},{ }^{15} \mathrm{~N}\right), \mathrm{E}=72 \mathrm{MeV}$; measured reaction $\sigma, \sigma(\theta)$ vS $Q$-value, $\left({ }^{16} \mathrm{O}\right)\left({ }^{16} \mathrm{O}\right)(\theta),\left({ }^{16} \mathrm{O}\right)(a)(\theta) .{ }^{18} \mathrm{O}$ levels deduced $\mathrm{J}$.

88Ba 13 V.G.Baty. E.A.Skakun, Yu.N.Rakivnenko, O.A.Rastrepin - Yad.Fiz. 47, 97B (1988) - Level-Density Spin Dependence for Nuclei Near the Closed Proton Shell at $3=50$

NUCLEAR HEACTIONS ${ }^{11} \mathrm{Cd}^{113} \mathrm{~J} n,{ }^{122} \mathrm{Sn}(\mathrm{p}, \mathrm{n}), \mathrm{E}=9 \mathrm{MeV}$; measured isomer pair production $\sigma$ ratios. ${ }^{210} \mathrm{Pd},{ }^{121} \mathrm{Sb}(\mathrm{p}, \mathrm{n}), \mathrm{E}$ not given; analyzed data; deduced level density spin dependence. ${ }^{111}$ In, ${ }^{122} \mathrm{Sb},{ }^{10} \mathrm{Ag},{ }^{121} \mathrm{Te}$ deduced moment of inertia.

88Ba18 V.G.Baty, E.A.Skakun, Yu.N.Rakivnenko, O.A.Rastrepin - Yad.Fiz. 47, 609 (1988) - Absolute and Relative Cross Sections of $(p, n)$-Reactions with Isomer Production in $\mathbf{A g}$ 3sotopes

NUCLEAR REACTIONS $104,105,108,100,110 \mathrm{Pd}(\mathrm{p}, \mathrm{n}), \mathrm{E}=5-9 \mathrm{MeV}$; measured $\sigma(\mathrm{E})$, relative,absolute isomer production $\sigma$. ${ }^{104} \mathrm{Ag}$ deduced moment of inertia,isomer $T_{1 / 2} \cdot{ }^{110} \mathrm{Ag}$ deduced moment of inertia.

88Ba29 F.C.Barker - Phys.Rev. C37, 2920 (1988) - Comment on ' Radiative Capture Reaction ${ }^{7} B e(p, \gamma)^{8} B$ at Low Energies NUCLEAR REACTIONS ' $\mathrm{Be}(\mathrm{p}, \gamma), \mathrm{E} \approx 2 \mathrm{MeV}$; calculated $\sigma(\mathrm{E})$. Direct,resonant capture.

88Ba30 A.Barbadoro, D.Consolaro, F.Pellegrini, G.F.Segato, l.Gabrielli - Phys.Rey. C38, 517 (1988) Excitation of Positive-Parity States in ${ }^{10} \mathrm{~B}$ with the ${ }^{13} \mathrm{C}(\mathrm{p}, \alpha)^{10} \mathrm{~B}$ Reaction NUCLEAR REACTIONS ${ }^{13} \mathrm{C}(p, p),(p, a), E=30.95 \mathrm{MeV}$; measured $\sigma(\theta)$; deduced $(p, a)$ reaction $\sigma(E)$. ${ }^{10} \mathrm{~B}$ levels deduced spectroscopic ractors. Triton cluster form factor.DWBA analysis.

88Ba52 V.G.Baty, E.A.Skakun, Yu.N.Rakivnenko, O.A.Rastrepin - Izv.Akad.Nauk SSSR, Ser.Fiz. 52, 94 (1988): Bull.Acad.Sci.USSR, Phys.Ser. 52, No.1, 88 (1988) - 199m,199te and 121m.1214Te Isomeric-Pair Excitation in Deuteron and a-Particle Reactions 
NUCLEAR REALTIONS ${ }^{118} \operatorname{Sn}(a, n),(a, 2 n),{ }^{117} \operatorname{Sn}(\alpha, 3 n),(\alpha, 2 n), E=t h r e s h o l d-32$ MeV; calculated $\sigma(E) .{ }^{11 \theta} \mathrm{Sn}(\alpha, n),(\alpha, 2 n), E=20-29 \mathrm{MeV} ;{ }^{12\rfloor} \mathrm{Sb}(d, 2 n), E \approx 4-16 \mathrm{MeV} ;$ calculated total $\sigma(E)$. ${ }^{121} \mathrm{Sb}(d, 2 \mathrm{n}), \mathrm{E}=4-16 \mathrm{MeV} ;{ }^{120} \mathrm{Sn}(\alpha, 3 \mathrm{n}), \mathrm{E}=29,3,31.7 \mathrm{MeV} ;{ }^{117,{ }^{118}} \mathrm{Sn}(\alpha, 2 \mathrm{n}),{ }^{116},{ }^{118} \mathrm{Sn}(\alpha, n), \mathrm{E}=16-32$ MeV; calculated isamer production orstios.

$88 \mathrm{Be} 06$

$88 \mathrm{~B} 101$

$88 B u 01$

$88 \mathrm{CaO} 04$

$88 \mathrm{ChOH}$

$88 \mathrm{Ch} 23$ M.B.Chatterjee
Lanthanum

NUCLEAR REACTIONS ${ }^{139} \mathrm{La}(\mathrm{p}, \mathrm{n} \gamma), \mathrm{E}=5.5 \mathrm{MeV}$; measured E $\gamma, \mathrm{l} \gamma, \gamma(\theta)$; deduced $\sigma(E) .{ }^{139} \mathrm{Ce}$ deduced levels,transition I $\gamma$,possible $\mathrm{J}, \pi$.

$88 \operatorname{col} 10$

P.Corvisiero, M.Anghinolfi, M.M.Giannini, G.Ricco, M.Sanzone, M.Taiuti - Nucl.Phys. A483, 9 (1988) - Proton and Deuteron Radiative Capture in Light Nuclei

NUCLEAR REACTIONS ${ }^{16} \mathrm{O}(\mathrm{p}, \gamma), \mathrm{E}=30 \mathrm{MeV} ;{ }^{15} \mathrm{~N}(\mathrm{~d}, \gamma), \mathrm{E}=16 \mathrm{MeV}$; measured capture $\sigma, \sigma(\theta)$; deduced $A_{0}, a_{1}$ coefficients. ${ }^{17} \mathrm{~F},{ }^{17} \mathrm{O}$ deduced GDR excitation mechanism. Gas targets,large anticoincidence $\mathrm{Nal}$ detector.

88 col2 M.E. colir, C.Friedli - Radiochim.Acta 43, 139 (1988) - Excitation Functions for Some Reactions Ir.duced by "B on Light Elements

NUCLEAR REACTIONS ${ }^{6} \mathrm{Li},{ }^{10} \mathrm{~B}\left({ }^{11} \mathrm{~B}, \mathrm{p}\right),{ }^{7} \mathrm{Li},{ }^{11} \mathrm{~B}\left({ }^{11} \mathrm{~B}, \mathrm{~d}\right),{ }^{9} \mathrm{Be}\left({ }^{11} \mathrm{~B}, 2 \mathrm{Z}\right),{ }^{28} \mathrm{Si},{ }^{24} \mathrm{Mg}\left({ }^{11} \mathrm{~B}, \mathrm{no}\right),{ }^{25} \mathrm{Mg},{ }^{29} \mathrm{Si}\left({ }^{11} \mathrm{~B}\right.$, $2 n \alpha),{ }^{26} \mathrm{Mg},{ }^{30} \mathrm{Si}\left({ }^{11} \mathrm{~B}, 3 \mathrm{na}\right),{ }^{9} \mathrm{Be}\left({ }^{11} \mathrm{~B}, 2 \mathrm{n}\right), \mathrm{E}=6-32 \mathrm{MeV}$; measured $\sigma(\mathrm{E})$, residual yields. Activation technique.

88De03

H.P.L.de Esch, C.van der Leun - Nucl.Phys. A476, 316 (1989) - Energy Levels of ${ }^{37} \mathrm{~K}$

NUCLEAR REACTIONS ${ }^{36} \mathrm{Ar}(\mathrm{p}, \gamma), \mathrm{E}=0.9-3.3 \mathrm{MeV} ;{ }^{36} \mathrm{Ar}\left(\mathrm{p}, \mathrm{p}^{\circ} \gamma\right), \mathrm{E}=2.6-3.3 \mathrm{MeV}$; measured $\sigma(\mathrm{E})$, $\sigma(\mathrm{E}(\gamma), \theta), \mathrm{E}(\gamma), \mathrm{I}(\gamma)(\theta)$; deduced $Q .{ }^{3{ }^{\prime} \mathrm{K}}$ deduced resonance energies, $\gamma$-branchings, J, $\pi, \delta, \mathrm{T}_{1 / 2}$, $\Gamma, \Gamma(\gamma) / \Gamma, \Gamma(\gamma) / \Gamma\left(p_{1}\right)$. Enriched,implanted targets.

88De07 M.De La Vega Vedoya, O.Capurro, A.Archentj, S.J.Nassiff - J.Radioanal.Nucl.Chem. 120. 49 (1988) - Investigation of $(\alpha, p \times n)$ Reactions on Natural Iron

NUCLEAR REACTIONS Fe $(a, \times n p){ }^{58} \mathrm{Co} /{ }^{37} \mathrm{Co} /{ }^{38} \mathrm{Co} /{ }^{58 m} \mathrm{Co} /{ }^{60} \mathrm{Co} /{ }^{60 m} \mathrm{Co}, \mathrm{E} \approx 4-90 \mathrm{MeV}$; measured residual production $\sigma(E)$, thick target yields.

88De 18 B.Dechant, E.Kuhlmann - 2.Phys. A330, 93 (1988) - Gammadecay of Quasimolecular Resonances in the
${ }^{12} \mathrm{C}+{ }^{12} \mathrm{C}$ Syslem 
NUCLEAR REACTIONS ${ }^{12} \mathrm{C}\left({ }^{12} \mathrm{C}, \gamma\right), \mathrm{E}(\mathrm{cm})=4.7-6 \mathrm{MeV}$; measured $\gamma$ yield, $\sigma(\theta)$ vs $\mathrm{E} .{ }^{12} \mathrm{C}\left({ }^{12} \mathrm{C}, a \gamma\right),{ }^{12} \mathrm{C}$, $\mathrm{P} \gamma),\left({ }^{12} \mathrm{C}, \mathrm{n} \gamma\right), \mathrm{E}(\mathrm{cm})=4.7-6 \mathrm{MeV}$; measured $\sigma(\mathrm{E})$; deduced $\gamma$-ray strength. ${ }^{24} \mathrm{Mg}$ resonances deduced $\Gamma$ (capture)/ $\Gamma$. Hauser Feshbach calculations. Natural targets. Nal spectrometer.

88De19 D.Degering, S.Untesricker, W.Stolz - J.Radjoasal.Nucl.Chem. 127, 7 (1988) - Excitation Fusction of the ${ }^{B Q} \mathrm{Y} / \mathrm{d}, 2 \mathrm{n} /{ }^{89} \mathrm{Zr}$ Reaction

NUCLEAA REACTIONS ${ }^{89} Y(d, 2 n), E<13.5 \mathrm{MeV} ;$ measured $\sigma(E)$; deduced reaction threshold. Stacking foil technique.

88De20

J.L.Q.de Britto, M.A.V.Bastos, R.f.da Silva, A.G.da Silva - J.Radioanal.Nucl.Chem. 127, 31 (1988) - Production of No Carrier Added Residue Free ${ }^{22} \mathrm{Na}$ NUCLEAR REACTIONS ${ }^{24} \mathrm{Mg}(d, \alpha), E=14 \mathrm{MeV}$; measured Er,l $\gamma$; deduced residual yield. Chemica! separation technique.

88De24 O.T.De Jesus, A.M.Friedman - Appl.Radiat.1sot. 39, $709(1988)$ - Production of ${ }^{79} \mathrm{Br} \mathrm{v}^{2} \mathrm{a}$ the ${ }^{78} \mathrm{Kr}(\mathrm{d}$, na) ${ }^{75} \mathrm{Br}$ Reaction

NUCLEAR REACTIONS ${ }^{78} \mathrm{Kr}(d, n a), E=21.5 \mathrm{MeV}$; measured residual production yield.

88Do0?

G.Doukellis - Phys.Rev. C37, 2233 (1988) - Fusion of ${ }^{12} \mathrm{~S}+{ }^{27} \mathrm{Al}$ and ${ }^{18} \mathrm{~F}+{ }^{40} \mathrm{Ca}$ and the Nucleus-Nucleus Potential

NUCLEAR REACTIONS ${ }^{27} \mathrm{Al}\left({ }^{32} \mathrm{~S}, \mathrm{X}\right), \mathrm{E}=67-393 \mathrm{MeV} ;{ }^{10} \mathrm{Ca}\left({ }^{10} \mathrm{~F}, \mathrm{X}\right), \mathrm{E} \approx 25-100 \mathrm{MeV}$; calculated fusion $\sigma(E)$; deduced fusion parametersnucleus-nucieus potential.

B8En03 S.Engstler, A.Krauss, K.Neldner, C.Rolfs, U.Schroder, K.Langanke - Phys.Let1. 202B, 179 (1988) Erfects of Electron Screening on the ${ }^{3} \mathrm{He}(\mathrm{d}, \mathrm{p})^{4} \mathrm{He}$ Low-Energy Cross Sections NUCLEAR REACTIONS ${ }^{3} \mathrm{He}(d, p), \mathrm{E}(\mathrm{cm})=5.9-41.6 \mathrm{keV}$; measured $\sigma(\mathrm{E})$. Atomic gas target.

$88 \mathrm{Fe07}$ I.D.fedorets, L.I.Zalyubovsky, B.A.Nemashkalo, V.E.Storizhko - Yad.Fiz. 48, 12 (1988) - ${ }^{77}$ Br Excitation Cross Sections in $(p, n \gamma)$ Reactions

NUCLEAR REACTIONS "7Se( $p, n \gamma), E \approx 2.8-3.5 \mathrm{MeV} ;$ measured $\sigma(\mathrm{E}), \gamma(\theta), \sigma(\mathrm{E} \gamma, \mathrm{E}) .{ }^{27} \mathrm{Br}$ deduced levels,decay reatures.

88 Fuoz C.Funck, K.Langanke - Nucl.Phys. A480, 188 (1988) - Microscopic Study of the ${ }^{14}$ o(a,p) ${ }^{17} \mathrm{~F}$ Reaction at Stellar Energies

NUCLEAR REACTIONS $14 \mathrm{O}(a, p) . E \approx 0.5-4 \mathrm{MeV}$; calculated reaction rate, reaction $\sigma$, astrophysical S-factor vs $\mathrm{E}$. IBNe deduced resonances, Generator coordinate method, r: ulti-channels.

$88 G a 01$

E.Gadioli, E.Gadjoli Eria, P.Guazzoni, L.Zetta - Phys.Rev. C37, 79 (1988) - Coherent Pickup and Knockout Contributions to the ${ }^{144} \mathrm{Nd}(\mathrm{p}, a)^{141} \mathrm{Pr}$ Reaction NUCLEAR REACTIONS $144 \mathrm{Nd}(p, a), E=25,35 \mathrm{MeV}$; analyzed $\sigma(\theta)$; deduced knockout contribution role,model parameters, $\sigma$.

88Gu01 M.Guillaume, C.De Landsheere, P.Rigo, R.Czichosz - Appl.Radiat.lsot. 39,97 (1988) - Automated Production of Postassium-38 for the study of Myocardial Perfusion using Positron Emission Tomography

NUCLEAR REACTIONS ${ }^{35} \mathrm{Cl}(a, n), E=15-30$ MeV; measured residual thick target yields.

88Gu07 I.F.Gutich, I.P.Okhrimenko - Yad.Fiz. 47, 1238 (1988) - Calculation of the Cross Section of Mirror Nuclear Reactions $d\left({ }^{3} \mathrm{H}, \mathrm{n}\right) a$ and $\mathrm{d}\left({ }^{3} \mathrm{H} \epsilon, \mathrm{p}\right) a$ at Subbarrier Energies NUCLEAR REACTIONS ${ }^{2} \mathrm{H}\left({ }^{3} \mathrm{He}, \mathrm{p}\right){ }^{2} \mathrm{H}(\mathrm{t}, \Omega), E=\mathrm{th}$ reshold- $0.6 \mathrm{MeV}$; calculated $\sigma(E), \mathrm{S}-\mathrm{factor}$ vs $\mathrm{E}$ ${ }^{5} \mathrm{Li}$ deduced resonance parameters.

88Hi06 M.Hilgemeier, H.W.Becker, C.Rolfs, H.P.Trautvetter, J.W.Hammer - Z.Phys. A329, 243 (1988) Absolute Cross Section of the ${ }^{3} \mathrm{He}(\alpha, \gamma)^{7} B e$ Reaction

NUCLEAR REACTIONS ${ }^{3} \mathrm{He}(\alpha, \gamma), \mathrm{E}(\mathrm{cm})=195-686 \mathrm{keV}$; measured $\sigma(E)$; deduced astropiyssical $\mathrm{S}(\mathrm{E})$ factor. Windowless gas targets, beam calorimeter,Ge detectors.

B81s01 M.1smajl, A.S.Divatia - Pramana 30, 193 (1988) - Measurement and Analysis of Alpha-1nduced Reactions on Ta,Ag and Co

NUCLEAR REACTIONS ${ }^{101} \mathrm{Ta}(\alpha, n),(\alpha, 2 n),(\alpha, 3 n),(\alpha, 4 n) \cdot{ }^{107} \mathrm{Ag}(\alpha, 4 n 2 p),{ }^{109} \mathrm{Ag}(\alpha, 2 n),{ }^{39} \mathrm{Co}(\alpha, 5 n 4 p),(\alpha$. $5 n 2 p),(a, 4 n 2 p),(a, 3 n 2 p),(a, n 2 p), E=60$ MeV; measured residual production $\sigma(E)$. Hybrid model.

8BIt01 M.G.Itkis, N.A.Kondratyev, Yu.V.Kotlov, S.I.Mulgin, V.N.Okolovich, A.Ya.Rusanov, G.N.Smirenkin Yad.fiz. 47, 7 (1988) - Low-Energy Fission of Gold Nuclei by Protons and a Particles NUCLEAR REACTIONS ${ }^{197} \mathrm{AU}(\alpha, F), E=35.9 \mathrm{MeV} ;{ }^{197} \mathrm{Au}(\mathrm{p}, \mathrm{F}), \mathrm{E}=26 \mathrm{MeV}$; measurad fission fragment massenergy distributions,yields. ${ }^{180} \mathrm{Kg}^{201} \mathrm{Tl}$ deduced fission properties. 
$88 \mathrm{Ka} 02$

88 Ka 07

88Ka 13

$8 B K j 01$

$88 \mathrm{KrOG}$

88Ku08

88La01

88La 15

88LeO4

88Le08

$88 M a 10$

88Mu 18

88Ma32 L.G.Mann, K.H.Maier, A.Aprahamian. J.A.Becker, D.J.Decman, E.A.Henry, R.A.Meyer, N.Roy, W.Stofrl,

T.Kajino, G.F.Bertsch, Ken-ichi Kubo - Phys.Rev. C37, 512 (1988) - E1 Polarizability of 'Li and Astrophysical S Factor for ${ }^{4} \operatorname{He}(t, \gamma)^{\mathrm{T}} \mathrm{Li}$

NUCLEAR REACTIONS ${ }^{58} \mathrm{Ni},{ }^{120} \mathrm{Sn}$ (polarized ${ }^{7} \mathrm{Li},{ }^{7} \mathrm{Li}$ ), E=sub-Coulomb; calculated $\mathrm{T}_{20}(\theta)$; deduced electric dipole polarizability. ${ }^{+} \mathrm{He}(\mathrm{t}, \gamma), \mathrm{E}(\mathrm{cm}) \approx 0-1.5 \mathrm{MeV}$; calculated radiative capture $\sigma, c h a n n e l$ dipole polarizability; deduced astrophysical $S$-factor $s(0)$.

T.Kajino, H.Toki, K.-I.Kubo, I.Tanihata - Phys.Lett. 202B, 475 (I988) - Nuclear-Matter Radii of

${ }^{3}$ Be and 'Li and Astrophysical S-Factors for Radjative Alpha-Capture Reactjons NUCLEAR REACTIONS ${ }^{3} \mathrm{H},{ }^{3} \mathrm{He}(\alpha, \gamma), \mathrm{E}=$ low; calculated capture $\sigma$, capture reaction astrophysical factor $S(E)$.

A.Kabir, M.W.Kermode, N.Rowley - Nucl.Phys. A481, 94 (1988) - Fusion Oscillations for ${ }^{16}$ O with ${ }^{12}$ C NUCLEAR REACTIONS ${ }^{16} \mathrm{O}\left({ }^{12} \mathrm{C}, \mathrm{X}\right), \mathrm{E}(\mathrm{cm}) \approx 7-27 \mathrm{MeV}$; calculated fusion $\sigma(E)$.

B.T.Kim, H.C.Kim, K.E.Park - Phys.Rev. C37, 998 (1988) - Heavy-Ion Optical Potential ror Sub-Barrier Fusion Deduced from a Dispersion Relation

NUCLEAR REACTIONS ${ }^{200} \mathrm{~Pb}\left({ }^{10} \mathrm{O}, \mathrm{X}\right), \mathrm{E} \approx 75-135 \mathrm{MeV}$; calculated rusion $\sigma(\mathrm{E})$; deduced optical potential parameters. Direct reaction model,fusion,dispersion relation

R.M.Kremer, C.A.Barnes, K.H.Chang, H.C.Evans, B.W.Filippone, K.H.Hahn, L.W.Mitchell Phys.Rev.Lett. 60, 1475 (1988) - Coincidence Measurement or the ${ }^{12} \mathrm{C}(\alpha, \gamma)^{18} \mathrm{O}$ Cross Section at Low Energies

NUCLEAR REACT1ONS ${ }^{12} \mathrm{C}(a, \gamma), \mathrm{E}(\mathrm{cm})=1.29-3$ MeV; measured $\gamma$-spectra, $\gamma($ recoil $)-$ coin; deduced $\gamma$ yields,dipole capture $\sigma(E), S-f a c t o r$ s $E$.

P.M.Kurjan, J.R.Calarco, G.A.Fisher, S.S.Hanna - Phys.Rev. C37, 2281 (1988) - Giant E1 Resonances in ${ }^{20} \mathrm{Ne}$ Observed $w \mathrm{jth}$ the ${ }^{i 9} \mathrm{~F}\left(\mathrm{p}(\mathrm{pol}), \gamma_{0} \gamma_{1}\right){ }^{20} \mathrm{Ne}$ Reactions

NUCLEAR REACTIONS ${ }^{19} \mathrm{~F}$ (polarized p, $\gamma$ ), $\mathrm{E}=3-10 \mathrm{MeV}$; measured $\gamma$ yield, $\sigma(\theta \gamma)$. ${ }^{20} \mathrm{Ne}$ deduced transition $\gamma$ multipolarity,GDR features.

M.C.Lagunas-Solar, O.F.Carvacho, R.R.Cima - Appl.Radiat.Isot. 39,41 (1988) - Cyclotron Production of PET Radionuclides: ${ }^{18}$ F(109.77 min; $\beta^{+} 96.9 \% ;$ EC $\left.3.1 \%\right)$ from High-Energy Protons on Metallic Aluminum Targets

NUCLEAR REACTIONS ${ }^{27}$ AJ(p,njp), $\left(p, 2_{n} 3 p\right),{ }^{27}$ Al(p,2n2a) ${ }^{18} F, E=13.3-67.5$ MeV; measured thin target,cumulative yields vs E. Activation,radiochemical techniques, ${ }^{18} \mathrm{~F}$ residue from ${ }^{10} \mathrm{Ne}$ decay.

R.M.Lambrecht, M.Sajjad, M.A.Qureshi, S.J.Al-Yanbawi - J.Radioanal.Nucl.Chem. 127, 143 (1988) Production of Iodin:-124 NUCLEAR REACTIONS $124 \mathrm{Te}(\mathrm{d}, 2 \mathrm{n}), \mathrm{E}=15 \mathrm{MeV}$; measured residual production yield

J.R.Leigh, J.J.M.Bokhorst, D.J.Hinde, J.O.Newton - J.Phys.(London) G14, L55 (1988) - observation or Sub-Barrier Fusion Enhancement Due to Negative Hexadecapole Deformations ( Question) NUCLEAR REACTIONS ${ }^{176,180} \mathrm{HF},{ }^{182},{ }^{184},{ }^{188} \mathrm{~W}\left({ }^{16} \mathrm{O}, \mathrm{X}\right), \mathrm{E}=70-90 \mathrm{MeV}$; measured fusion $\sigma(\mathrm{E})$. 150,152 ${ }^{15} \mathrm{Sm}\left({ }^{10} \mathrm{O}, \mathrm{X}\right),{ }^{181} \mathrm{Ta},{ }^{159} \mathrm{~Tb},{ }^{139} \mathrm{La},{ }^{109} \mathrm{Tm}\left({ }^{10} \mathrm{~F}, \mathrm{X}\right), \mathrm{E}$ not given; calculated fusion $\sigma(\mathrm{E})$. ${ }^{176},{ }^{180} \mathrm{Hr},{ }^{182}$, $184,106 \mathrm{~W}$ deduced hexadecapole deformation nature.

K.T.Lesko, E.B.Norman, R.-M.Larimer, S.Kuhn, D.M.Meekhof, S.G.Crane, H.G.Bussell - Phys.Rev. C37 1808 (1988) - Measurements of Cross Sections Relevant to $\gamma$-Ray Line Astronomy NUCLEAR REACTIONS $\mathrm{C}, \mathrm{N}, \mathrm{O}, \mathrm{Mg},{ }^{27} \mathrm{Al}, \mathrm{Si}, \mathrm{Fe}(\mathrm{p}, \mathrm{xnypza}), \mathrm{E}=8.9-50 \mathrm{MeV}$; measured $\gamma-\mathrm{production} \sigma(\mathrm{E})$.

W.-H.Ma, S.-P.Zhao, Z.-Y.Feng, C.-K.Chen - Nucl.Phys. A477, 713 (1988) - Energy Dependence of Heavy Ion Collisions ${ }^{4} \mathrm{He}+{ }^{12} \mathrm{C}$ and ${ }^{12} \mathrm{C}+{ }^{12} \mathrm{C}$ Elastic Scattering

NUCLEAR REACTJONS ${ }^{12} \mathrm{C}(\alpha, a),{ }^{12} \mathrm{C}\left({ }^{12} \mathrm{C},{ }^{12} \mathrm{C}\right), \mathrm{E}(\mathrm{cm}) \approx 5-2000 \mathrm{MeV} /$ nucleon; calculated total, reaction $\sigma(E)$. Glauber theory. G.L.Struble - Phys.Rev. C38, 74 (1988) - Levels of the Four Lowest Two-Particle Configurations in ${ }^{210} \mathrm{Po}$ studied by In-Beam $\gamma$-Ray and Conversion-Electron Spectroscopy with the ${ }^{20}$ Bi(t,2n) Reaction

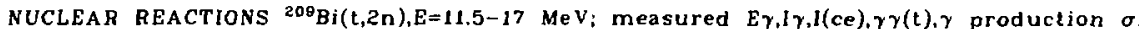
$210^{\circ}$ deduced levels J, $\pi, \gamma$-branching, ICC, $\gamma$-multipolarity, configurations. Shell model.

M.G.Mustafa, H.I.West,Jr., H.o'Brien, R.G.Lanier, M.Eenhamou, T.Tamura - Phys.Rev. C38, l624 (1988) - Measurements and a Direct-Reaction - Plus - Hauser-Feshbach Analysis of ${ }^{89} Y(p, n)^{89} Z{ }^{8}$, ${ }^{80} \mathrm{Y}(\mathrm{p}, 2 \mathrm{n})^{\theta \theta \mathrm{Z} r}$, and ${ }^{\theta 8} \mathrm{Y}(\mathrm{p}, \mathrm{pn})^{\theta \theta} \mathrm{Y}$ Reactions up to $40 \mathrm{MeV}$ 
NUCLEAR REACTIONS ${ }^{B \theta} \mathrm{Y}(p, n),(p, 2 n),(p, n p), E=4.59-39.56$ MeV; measured $\sigma(E)$; deduced reaction mechanism. Foil activation method. Hauser-Feshbach calculation,one-two-step direct reactions,preequilibrium model analyses.

$88 \mathrm{PaO} 4$

$88 Q \mathrm{a} 02$

$88 \mathrm{Sc08}$

$885 i 10$

N.L.Singh, A.V.Mohan Rao, S.Mukherjee, R.Upadhyay, R.K.Jain, S.K.Bose, L.Chaturvedi, J.Rama Rao - J.Phys.(London) G14, 931 (1988) - Alpha-Induced Reactions on Gold

NUCLEAR REACTIONS ${ }^{197} \mathrm{~A} u(\alpha, n),(\alpha, 2 n),(\alpha, 3 n),(\alpha, n 2 p),(\alpha, n \alpha),(\alpha, 3 n \alpha), E=$ threshold -75 MeV; measured $\sigma(E)$. Hybrid model.

$\operatorname{8gsm} 01$

M.J.Smithson, D.L.Watson, H.T.Fortune - Phys.Rev. C37, $1036(1988)-{ }^{13} \mathrm{C}\left({ }^{6} \mathrm{Li}, \mathrm{p}\right){ }^{18} \mathrm{O}$ Reaction at $E\left({ }^{6} \mathrm{Li}\right)=28 \mathrm{MeV}$

NUCLEAR REACTIONS ${ }^{13} \mathrm{C}\left({ }^{6} \mathrm{Li}, \mathrm{p}\right), \mathrm{E}=28 \mathrm{MeV}$; measured $\sigma(\mathrm{Ep}), \sigma(\theta)$; deduced model parameters, $\sigma(E) .{ }^{18} O$ deduced levels, $\mathrm{J}, \pi$.

$8 \mathrm{BSr} 02$

D.K.Srivastava, D.N.Basu, H.Rebe1 - Nucl.Phys. A485, 221

Potential from Coulomb Dissociation of Deuterons and ${ }^{3} \mathrm{He}$

NUCLEAR REACT1ONS ${ }^{208} \mathrm{~Pb}(\mathrm{~d}, \mathrm{np}) .\left({ }^{3} \mathrm{He}, \mathrm{pd}\right), \mathrm{E} \approx 10-250 \mathrm{MeV}$; calculated Coulomb dissociation $\sigma(E)$. Dynamic polarization.

88503

F.Sterba, J.Liptak, Nguyen An Ninh, J.Sterbova of $1805 \mathrm{keV}$ Resonance in $p+{ }^{55} \mathrm{Mn}$ interaction NUCLEAR REACTIONS ${ }^{55} \mathrm{Mn}\left(p, p^{\prime} \gamma\right),(p, n \gamma),(p, \gamma), E=1.3-2 \mathrm{MeV}$; measured $\gamma$ yields, $\sigma(E)$. SF Pe deduced resonance,possible $J, \pi$.

88St 14 F.Sterba, J.Liptak, Nguyen An Ninh, J.Sterbova, J.Stary - Czech.J.Phys. B38, 741 (1988) Excitation Functions for Some Output Channels folJowing the $p+{ }^{55} \mathrm{Mn}$ Interaction at $E(p)=$ $1.3-2.0 \mathrm{MeV}$

NUCLEAR REACTIONS ${ }^{35} \mathrm{Mn}\left(\mathrm{p}, \mathrm{p}^{\prime}\right),(\mathrm{p}, \mathrm{n} \gamma),(\mathrm{p}, \gamma), \mathrm{E}=1.3-2 \mathrm{MeV}$; measured $\sigma(\mathrm{E}), \gamma$ yields. ${ }^{56} \mathrm{Fe}$ deduced resonances, $J, \pi$

$88 \mathrm{Ta} 02$

F.Tarkanyi, S.M.Qaim, G.Stocklin - Appl.Radiat.lsot. 39, 135 (1988) - Exication Functions of ${ }^{3}$ Heand a-Particle Induced Nuclear Reactions on Natural Krypton: Production of ${ }^{82} \mathrm{Sr}$ at a compact cyclotron

NUCLEAR REACTIONS $\mathrm{Kr}\left({ }^{3} \mathrm{He}, x n\right)^{82} \mathrm{Sr} /{ }^{83} \mathrm{Sr} /{ }^{83} \mathrm{Sr} /{ }^{85 m^{5}} \mathrm{~S} r /{ }^{87} \mathrm{Sr} /{ }^{87 m} \mathrm{Sr}, K r\left({ }^{3} \mathrm{He}, x n \mathrm{np}\right)^{81} \mathrm{Rb} /{ }^{82} \mathrm{Rb} /{ }^{82} \mathrm{~m} \mathrm{Rb} /{ }^{83} \mathrm{Rb} /$

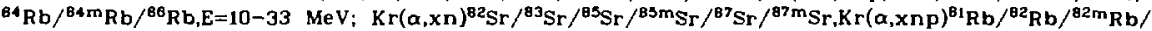
${ }^{83} \mathrm{Rb} /{ }^{84} \mathrm{Rb} /{ }^{86} \mathrm{Rb}, \mathrm{E}=10-25 \mathrm{MeV}$; measured residual production $\sigma(E)$, thick target yields. $\mathrm{Kr}\left({ }^{3} \mathrm{He}\right.$, $\mathrm{xn}^{\mathrm{B2}} \mathrm{Sr} /{ }^{83} \mathrm{Sr} /{ }^{85} \mathrm{Sr}, \mathrm{E} \approx 10-35 \mathrm{MeV}$; calculated residual production $\sigma(E)$.

$88 \mathrm{Ti} 0$
R.Timmermann, H.W.Becker, C.Rolfs, U.Schroder, H.P.Trautvetter - Nucj.Phys. A477, 105 (1988) Search for Low-Energy Resonances in ${ }^{27} \mathrm{Al}(\mathrm{p}, \alpha)^{24} \mathrm{Mg}$ 
NUCLEAR REACTIONS ${ }^{27} \mathrm{Al}(\mathrm{p}, \alpha), \mathrm{E}=200-360 \mathrm{keV}$; measured $\sigma(\mathrm{E}(\alpha)),{ }^{27} \mathrm{Al}(\mathrm{p}, \gamma), \mathrm{E}=200-360^{\circ} \mathrm{keV}$; measured thick target $\gamma-y$ ields; deduced astrophysical reaction rates. ${ }^{2 \theta}$ Si deduced resonance strengths. Targets of low boron contamination.

88Ti03 C.I.W.Tingwell, V.Y.Hansper, S.G.Tims, A.F.Scott, D.G.Sargood - Nucl.Phys, A480, 162 (1988) Cross Sections of Proton Induced Reactions on ${ }^{61} \mathrm{Ni}$ NUCLEAR REACTIONS ${ }^{61} \mathrm{Ni}(\mathrm{p}, \gamma),\left(p_{1} \mathrm{p}^{\prime} \gamma\right), \mathrm{E}=1.05-3.98 \mathrm{MeV} ;{ }^{61} \mathrm{Ni}(\mathrm{p}, \mathrm{n}), \mathrm{E}=3.08-4.66 \mathrm{MeV}$; measured

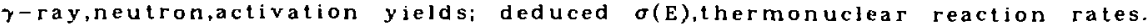
Statistical-model analysis. Enriched target.

8ETi 05

S.G.Tims, C.I.W.Tingwell, V.Y.Hansper, A.F.Scott, D.G.Sargood - Nucl.Phys. A483, 354 (1988) The ${ }^{59} \mathrm{Co}(\alpha, p)^{62} \mathrm{Ni}$ and ${ }^{50} \mathrm{Co}(\alpha, n)^{62} \mathrm{Cu}$ Cross Sections NUCLEAR REACTIONS ${ }^{30} \mathrm{Co}(a, p), E=5,18-9.4 \mathrm{MeV} ;{ }^{50} \mathrm{Co}(a, n), \mathrm{E}=5.07-9.8 \mathrm{MeV}$; measured proton, neutronactivation yields; deduced $\sigma(E)$,thermonuclear reaction rates. Natural targets.

88Tr01 S.Trentalange, S.-C.Wu, J.L.Osborne, C.A.Barnes - Nucl.Phys, A483, 406 (I988) - Elastic Scattering and Fusion Cross Sections of ${ }^{13} \mathrm{C}+{ }^{13} \mathrm{C}$

NUCLEAR REACTIONS ${ }^{13} \mathrm{C}\left({ }^{13} \mathrm{C}, \mathrm{X}\right), \mathrm{E}(\mathrm{cm})=3.26-8 \mathrm{MeV}$; measured $\sigma, \gamma$-ray yields from ${ }^{18} \mathrm{O}^{21},{ }^{22} \mathrm{Ne}$, ${ }^{24} \mathrm{Na},{ }^{24},{ }^{25} \mathrm{Mg}$; deduced total fusion o,elastic $\sigma(E), \sigma(\theta)$. Optical,incoming wave boundary condition models.

88Ts01 I.Tserruya, V.Steiner, Z.Fraenkel, P.Jacobs, D.G.Kovar, W.Henning, M.F.vineyard, B.G.Glagola Phys.Revilett. 60, 14 (1988) - Incomplete Fusion Reactions Induced by ${ }^{12} \mathrm{C}$ at $5.5-10$ MeV/Nucleon NUCLEAR REACTIONS ${ }^{120} \mathrm{Sn},{ }^{160} \mathrm{Gd},{ }^{197} \mathrm{Au}\left({ }^{12} \mathrm{C}, \mathrm{X}\right), E=5.5-10 \mathrm{MeV} / \mathrm{nucleon}$; measured complete, incomplete fusion $\sigma(E), \sigma(\theta)$.

88 Va 14

M.E.Vazquez, M.J.ozafran, M.de la Vega Vedoya, S.J.Nassiff - J.Radioanal.Nucl.Chem. 121,203 (1988) - $(\alpha, \alpha \times n)$ and $(\alpha, 2 p \times n)$ Reactions on ${ }^{93} \mathrm{Nb}$ NUCLEAR REACTIONS ${ }^{93} \mathrm{Nb}(\alpha, x n \alpha),(\alpha, x n 2 \mathrm{p})^{95} \mathrm{Nb} /{ }^{95 \mathrm{~m}} \mathrm{Nb} /{ }^{92} \mathrm{Nb} /{ }^{92 \mathrm{~m}} \mathrm{Nb} /{ }^{91} \mathrm{Nb} /{ }^{91 \mathrm{~m}} \mathrm{Nb} /{ }^{90} \mathrm{Nb} /{ }^{90 \mathrm{~m}} \mathrm{Nb}, \mathrm{Es} 90 \mathrm{MeV}$; measured residual production $\sigma(E)$; deduced cumulative $\sigma(\theta)$, thick target yields.

88\%a02 B.Wachter, T.Mertelmeier, H.M.Hofmann - Phys.Lett. 200B, 246 (1988) - The ${ }^{2} \mathrm{H}(\mathrm{d}, \gamma)^{4} \mathrm{He}$ Reaction and the D-State of the Alpha Particle: A microscopic study NUCLEAR REACTIONS ${ }^{2} \mathrm{H}$ (polarized d, $\gamma$ ),E=10 MeV; calculated $\sigma(\theta)$,analyzing powers,capture $\sigma$, astrophysical $S(E)$. Microscopic multi-channel resonating group model.

88Wa 1 C.Wasilersky, M.J.Ozàfran, M.de la Vega Vedoya, S.J.Nassiff - J.Radioanal.Nucl.Chem. 121, 115 (1988) - $(\alpha, \alpha \times n)$ and $(\alpha, 2 \mathrm{p} \times \mathrm{n})$ Reactions on ${ }^{107} \mathrm{Ag}$ and ${ }^{109} \mathrm{Ag}$

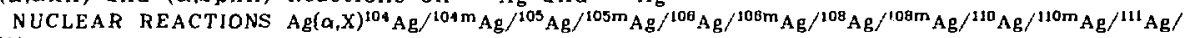
${ }^{111 \mathrm{~m} A g, E} \leq 90 \mathrm{MeV}$; measured residual production $\sigma(E)$. Activation technique.

88Wa 13 T.R.Warg, W.Haeberli, S.W.Wissink, S.S.Hanna - Phys.Rev. C37, 2301 (1988) - Electrio Quadrupole Strength in ${ }^{20} \mathrm{Ne}$ from the ${ }^{10} \mathrm{~F}\left(\mathrm{p}(\mathrm{pol}), \gamma_{0}\right)^{20} \mathrm{Ne}$ Reaction

NUCLEAR REACTIONS ${ }^{19} \mathrm{~F}$ (polarized $\left.\mathrm{p}, \gamma\right), E=3.5-13.3 \mathrm{MeV}$; measured $\sigma(\theta)$, analyzing power vs $\theta$, $\sigma(E) .{ }^{20} \mathrm{Ne}$ deduced $\mathrm{CQR}$ fealures.

88Wi08 M.Wiescher, J.Gorres, F.-K.Thielemann - Astrophys.J. 326, $384(1988)-{ }^{37} \mathrm{~F}(\mathrm{p}, \gamma)^{16} \mathrm{Ne}$ in Explosive Hydrogen Burning

NUCLEAR REACTIONS ${ }^{17} \mathrm{~F}(\mathrm{p}, \gamma), \mathrm{E}(\mathrm{cm}) \leq 0.7 \mathrm{MeV}$; calculated capture $\sigma, S-f a c t o r$ vs E. ${ }^{18} \mathrm{Ne}$ deduced level,J, $\pi$.

88Wil6 S.W.Wissink, S.S.Hanna, D.G.Mavis, T.R.Wang - Phys.Rev, C37, 2289 (1988) - Electric Quadrupole Strength in ${ }^{16} \mathrm{O}$ from the ${ }^{15} \mathrm{~N}\left(\mathrm{p}(\mathrm{pol}), \mathrm{y}_{0}\right)^{16} \mathrm{O}$ Reaction

NUCLEAR REACTIONS ${ }^{15} \mathrm{~N}$ (polarized $\left.p, \gamma\right), E=6.25-13.75 \mathrm{MeV}$; measured $\gamma(\theta)$, analyzing powers, $\sigma(\theta), \sigma(E) .{ }^{10} O$ deduced $G Q R$ features. 


\section{TARGET REACTION}

$\begin{array}{cl}{ }^{1} H & (d, d) \\ & \left({ }^{11} B, a\right) \\ & \\ { }^{2} H \quad & (d, \gamma) \\ & (d, \gamma) \\ & (d, \gamma) \\ & (d, \gamma) \\ & (d, \gamma) \\ & (d, n) \\ & (d, p) \\ & (d, p) \\ & (d, p) \\ & (d, d) \\ & \left(d,{ }^{3} H e\right) \\ & (t, n) \\ & (t, n) \\ & \left({ }^{3} \mathrm{He}, p\right)\end{array}$

${ }^{3} \mathrm{H} \quad(d, n)$

$(\mathrm{d}, \mathrm{d})$

$(d, \alpha)$

$(\alpha, \gamma)$

$(\alpha, \gamma)$

$(\alpha, \gamma)$

$(\alpha, \gamma)$

${ }^{3} \mathrm{He} \quad(d, p)$

(d,p)

(d,p)

$(d, p)$

( $\left.{ }^{3} \mathrm{He}, 2 \mathrm{p}\right)$

( $\left.{ }^{3} \mathrm{He}, 2 \mathrm{p}\right)$

( $\left.{ }^{3} \mathrm{He}, 2 \mathrm{p}\right)$

$\left({ }^{3} \mathrm{He}, \mathrm{n} 2 \mathrm{p}\right)$

( ${ }^{3} \mathrm{He}, \mathrm{pd}$ )

$\left({ }^{3} \mathrm{He}, 3 \mathrm{p}\right)$

( ${ }^{3} \mathrm{He}$, 2pd)

( $\left.{ }^{3} \mathrm{He}, \mathrm{X}\right)$

$(a, \gamma)$

$(\alpha, \gamma)$

${ }^{4} \mathrm{He} \quad(t, \gamma)$

${ }^{\theta} \mathrm{Li} \quad\left({ }^{\theta} \mathrm{Li}, \mathrm{n}\right)$

( $\left.{ }^{8} L i, p\right)$

( $\left.{ }^{\theta} L i, n p\right)$

( ${ }^{8} \mathrm{Li}, \mathrm{d}$ )

( $\left.{ }^{11} B, p\right)$

$\left({ }^{12} \mathrm{C}, \mathrm{X}\right)$

$\left({ }^{18} 0 . n\right)$

$\left({ }^{18} \mathrm{O}, \mathrm{p}\right)$

( $\left.{ }^{18} \mathrm{O}, \mathrm{p}\right)$

$\left({ }^{18} \mathrm{O}, \mathrm{d}\right)$

$\left({ }^{18} \mathrm{O},{ }^{3} \mathrm{He}\right)$

$\left({ }^{10} 0, a\right)$

$\left({ }^{10} \mathrm{O}, \mathrm{na} a\right)$

$\left({ }^{10} 0, p a\right)$

$\left({ }^{18} 0,2 a\right)$

${ }^{7} \mathrm{Li} \quad(p, \gamma)$

$(p, n)$

$(p, a)$

$(t, n)$

$(t, p)$

("1 $B, d)$
INCIDENT ENERGY

$E \leq 20 \mathrm{keV}$

$E=1.68-13.2 \mathrm{MeV}$

$\mathrm{E}(\mathrm{cm})=0.010-10 \mathrm{MeV}$

$E=0.075-3 \mathrm{MeV}$

$E(\mathrm{~cm})=0.010-10 \mathrm{MeV}$

$\mathrm{E}(\mathrm{cm})=50-500 \mathrm{keV}$

$E(\mathrm{~cm})=2.98-162.5 \mathrm{keV}$ $\mathrm{E} \leq 50 \mathrm{keV}$

$E(\mathrm{~cm})=2.98-162.5 \mathrm{keV}$

$E=20-11.7 \mathrm{keV}$

$\mathrm{E} \leq 20 \mathrm{keV}$

$E=20-117 \mathrm{keV}$

$\mathrm{E}=\mathrm{th}$ reshold $-0.6 \mathrm{MeV}$

$\mathrm{E}=0-300 \mathrm{keV}$

$E=$ threshold-0.6 MeV

$E=1 \mathrm{keV}-10 \mathrm{MeV}$

$\mathrm{E} \leq 20 \mathrm{keV}$

$E=80-116 \quad \mathrm{keV}$

$E=10 w$

$E(\mathrm{~cm})=79-464 \mathrm{keV}$

$E=0.7-2 \mathrm{MeV}$

$E \approx 50-600 \mathrm{keV}$

$\mathrm{E} \leq 50 \mathrm{keV}$

$E(\mathrm{~cm})=6.95-141.8 \mathrm{keV}$

$\mathrm{E}=0.001-1 \mathrm{MeV}$

$E(\mathrm{~cm})=5.9-41.6 \mathrm{keV}$

$\mathrm{E} \leq 250 \mathrm{keV}$

$E(\mathrm{~cm})=17.9-342.5 \mathrm{keV}$

$\mathrm{E}=17.9,21.7,24 \mathrm{MeV}$

$E=17.9,21.7,24 \mathrm{MeV}$

$\mathrm{E}=17.9,21.7,24 \mathrm{MeV}$

$E=17.9,21.7,24 \mathrm{MeV}$

$E=17.9,21.7,24 \mathrm{MeV}$

$E=17.9,21.7,24 \mathrm{MeV}$

$E(\mathrm{~cm})=195-686 \mathrm{keV}$

$E=10 w$

$\mathrm{E}(\mathrm{cm}) \approx 0-1.5 \mathrm{MeV}$

$E=2-16 \mathrm{MeV}$

$\mathrm{E}=2-16 \mathrm{MeV}$

$E(\mathrm{~cm})=7.2-13.3 \mathrm{MeV}$

$E(\mathrm{~cm})=7.2-13.3 \mathrm{MeV}$

$E=6-32 \mathrm{MeV}$

$E=60 \mathrm{MeV}$

$E=10-40 \mathrm{MeV}$

$E=10-40 \mathrm{MeV}$

$E=10-40 \mathrm{MeV}$

$\mathrm{E}=10-40 \mathrm{MeV}$

$E=10-40 \mathrm{MeV}$

$E=10-40 \mathrm{MeV}$

$E=10-40 \mathrm{MeV}$

$E=10-40 \mathrm{MeV}$

$E=10-40 \mathrm{MeV}$

$E=7-9 \mathrm{MeV}$

$\mathrm{E}=7-9 \mathrm{MeV}$

$E \leq 250 \mathrm{keV}$

$E \approx 5.7-8.5 \mathrm{MeV}$

$E \approx 5.7-8.5 \mathrm{MeV}$

$E=6-32 \mathrm{MeV}$
REFERENCE

KEY NUMBER

JoUR Phys.tett. 202, 289

JOUR Z.Phys. A327, 341

BEBe06

B7Be17

JOUR Phys.Rev. C36, 21

JOUR Phys.Rev, C36, B75

JOUR Phys.Rev. C36, 17

JOUR Phys.Lett, 197, 315

JoUR Phys.Lett. 200, 246

JOUR Nuci.Phys. A 465, 150

JOUR Z.Phys. A327, 461

JOUR Nucl.Phys. A 465, 150

YOUR Nucl.lnstr.Meth. Phys.Res. B10/11, 405

JOUR Phys.Lett. 202, 289

JoUR Nucl.Instr.Meth. Phys.Res. B10/11, 405

JOUR Yad.Fiz. 47, 1238

JOUR Yad.Fiz. 46, 757

JOUR Yad.Fiz. 47, 1238

JOUR At.Energ. 63, 252

JouR Phys.Lett. 202, 289

JOUR Phys.Rev, C35, 1999

JOUR Phys.lett. 202, 475

JoUR Phys.Lett. 142, 55

JOUR Nucl.Phys. A473, 179

JOUR Z.Phys. A330, 277

878112

$87 \mathrm{Pi} 08$

87 A 03

87 Ba 58

$88 \mathrm{WaO} 02$

$87 K 518$

87As05

$87 \mathrm{Kr} 18$

85Ja 16

$88 \mathrm{Be} 06$

$B 5 \mathrm{~J}$ a 16

$88 \mathrm{Gu07}$

B7Va 36

88Gu07

$87 \mathrm{Gu} 25$

$88 \mathrm{Be} 06$

$87 \mathrm{Br} 10$

$88 \mathrm{Ka} 07$

$87 \mathrm{Sc} 18$

$87 \mathrm{Bu} \perp \mathrm{B}$

$88 \mathrm{~A} 124$

JoUR Z.Phys. A327, 461

JOUR Nuc1.Phys. A465, 150

JOUR At.Energ. 63, 252

JOUR Phys.Lett, 202, 179

JOUR Z.Phys. A327, 461

JOUR Nucl.Phys. A467, 273

JOUR Phys.Rev. C35, 383

JOUR Phys.Rev. C35, 383

JOUR Phys.Rev. C35, 383

JOUR Phys,Rev. C35, 383

JoUR Phys.Rev. C35, 383

JOUR Phys.Rev. C35, 383

JOUR Z.Phys. A329, 243

JoUR Phys.Lett. 202, 475

JOUR Phys.Rev, C37, 512

87 As 05

$87 \mathrm{~K}+18$

$87 \mathrm{Gu} 25$

$88 \mathrm{En} 03$

B7As05

$87 \mathrm{KrO9}$

$87 \mathrm{Br} 02$

$87 \mathrm{Br} 02$

87 Br02

$87 \mathrm{Br} 02$

87Bro2

87 Bro2

$88 \mathrm{H}$ i 06

$88 \mathrm{Ka07}$

$88 \mathrm{KaO}$

JOUR Nucl.Phys. A467, 149

87Do05

B7Do0s

JOUR Nucl.Phys. A467, 149

87 Arí3

87 A 13

BBCol2

$87 \mathrm{~Pa} 12$

B7Di07

87Di07

87Di07

87Di07

87Di07

87Di07

87Di07

87Di 07

870107

JoUR Radiochim. Acta 42,1

$87 \mathrm{Ra} 23$

87 R 23

87As05

B7Ab15

87Ab15

B8Cal2 


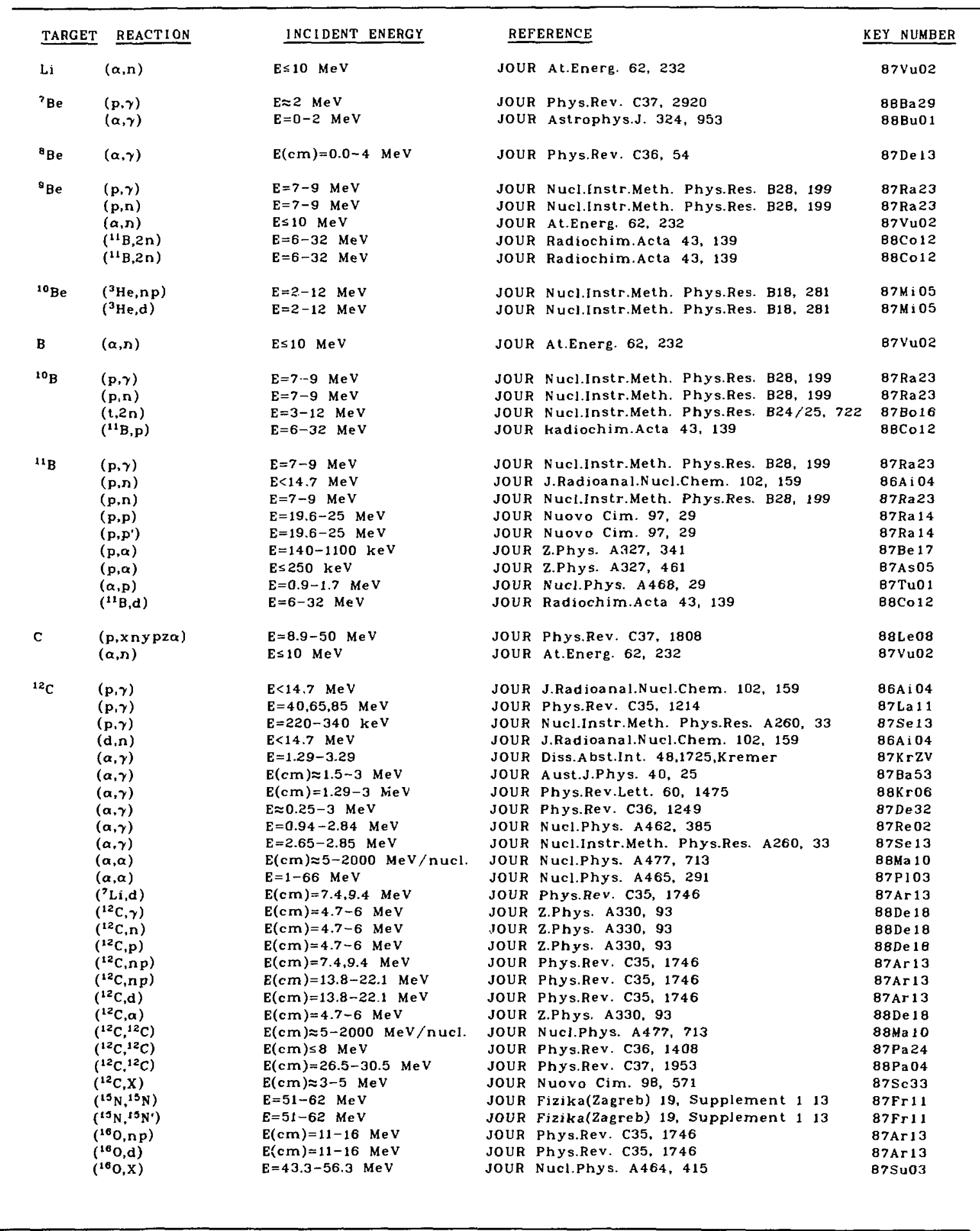




\begin{tabular}{|c|c|c|}
\hline TARGET & REACTION & INCIDENT ENERCY \\
\hline${ }^{13} \mathrm{C}$ & $\begin{array}{l}(p, n) \\
(p, p) \\
(p, \alpha) \\
(a, n) \\
(\alpha, a) \\
\left({ }^{6} 1, p\right) \\
\left({ }^{13} \mathrm{C}, X\right) \\
\left({ }^{16} 0, n p\right) \\
\left({ }^{16} 0, d\right)\end{array}$ & $\begin{array}{l}E<14.7 \mathrm{MeV} \\
E=30.95 \mathrm{MeV} \\
E=30.95 \mathrm{MeV} \\
E=10 \mathrm{w} \\
E=10 \mathrm{w} \\
E=28 \mathrm{MeV} \\
E(\mathrm{~cm})=3.26-8 \mathrm{MeV} \\
E(\mathrm{~cm})=11-16 \mathrm{MeV} \\
E(\mathrm{~cm})=11-16 \mathrm{MeV}\end{array}$ \\
\hline${ }^{14} \mathrm{C}$ & $\begin{array}{l}(\alpha, \gamma) \\
(\alpha, \gamma)\end{array}$ & $\begin{array}{l}\mathrm{E}=1.7-2.7 \mathrm{MeV} \\
\mathrm{E}=0-2 \mathrm{MeV}\end{array}$ \\
\hline$N$ & $(p, x \cap y p z \alpha)$ & $E=8.9-50 \mathrm{MeV}$ \\
\hline${ }^{13} \mathrm{~N}$ & $(p, \gamma)$ & $E=30 W$ \\
\hline${ }^{14} \mathrm{~N}$ & $\begin{array}{l}(p, \gamma) \\
(p, \gamma) \\
(p, \gamma) \\
(p, \gamma) \\
(p, n) \\
(p, a)\end{array}$ & $\begin{array}{l}\mathrm{E}=0.5-3.5 \mathrm{MeV} \\
\mathrm{E}=260-360 \mathrm{keV} \\
\mathrm{E}=0.2-3.6 \mathrm{MeV} \\
E=7-9 \mathrm{MeV} \\
E=7-9 \mathrm{MeV} \\
E<14.7 \mathrm{MeV}\end{array}$ \\
\hline${ }^{15} \mathrm{~N}$ & $\begin{array}{l}(p, \gamma) \\
(p, a) \\
(d, \gamma)\end{array}$ & $\begin{array}{l}E=6.25-13.75 \mathrm{MeV} \\
E \leq 250 \mathrm{keV} \\
E=16 \mathrm{MeV}\end{array}$ \\
\hline 0 & $(p, x \cap y p z \alpha)$ & $E=8.9-50 \mathrm{MeV}$ \\
\hline 140 & $\begin{array}{l}(a, \gamma) \\
(a, p) \\
(a, p)\end{array}$ & $\begin{array}{l}E \text { not given } \\
E \text { not given } \\
E \approx 0.5-4 \mathrm{MeV}\end{array}$ \\
\hline${ }^{15} \mathrm{O}$ & $(\alpha, \gamma)$ & E not given \\
\hline${ }^{16} \mathrm{O}$ & $\begin{array}{l}(p, \gamma) \\
(p, \gamma) \\
(p, \gamma) \\
(p, n) \\
(p, a) \\
(d, n) \\
(t, n) \\
(t, n) \\
(a, \gamma) \\
(a, \gamma) \\
(a, \gamma) \\
(a, X) \\
\left({ }^{6} \mathrm{Li}, n p\right) \\
\left({ }^{6} \mathrm{Li}, \mathrm{d}\right) \\
\left({ }^{12} \mathrm{C},{ }^{12} \mathrm{C}\right) \\
\left({ }^{12} \mathrm{C}, \mathrm{X}\right) \\
\left({ }^{12} \mathrm{C}, \mathrm{X}\right) \\
\left({ }^{16} \mathrm{O}, \mathrm{np}\right) \\
\left({ }^{18} \mathrm{O}, \mathrm{d}\right) \\
\left({ }^{16} \mathrm{O}, \alpha\right) \\
\left({ }^{16} \mathrm{O},{ }^{12} \mathrm{C}\right) \\
\left({ }^{18} \mathrm{O},{ }^{15} \mathrm{~N}\right) \\
\left({ }^{18} \mathrm{O},{ }^{10} \mathrm{O}\right) \\
\left({ }^{18} \mathrm{O}, \mathrm{X}\right) \\
\left({ }^{16} \mathrm{O}, \mathrm{X}\right) \\
\left({ }^{18} \mathrm{O}, \mathrm{X}\right) \\
\left({ }^{10} \mathrm{O}, \mathrm{X}\right) \\
\left({ }^{16} \mathrm{O}, \mathrm{X}\right)\end{array}$ & 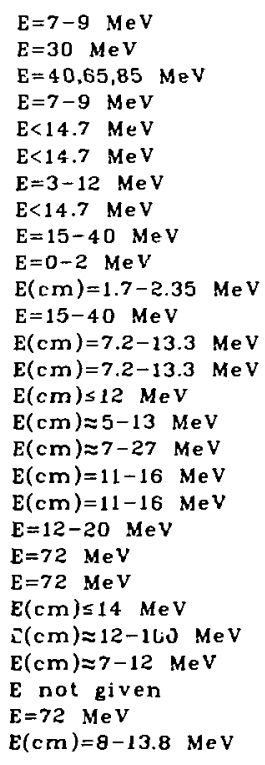 \\
\hline
\end{tabular}

\section{REFERENCE}

JOUR J.Radioanal.Nucl.Chem. 102, 159

JOUR Phys.Rev. C38, 517

JOUR Phys.Rev. C38, 517

JouR Phys.Rev. C36, 2206

JOUR Phys.Rev. C36, 2206

JoUR Phys.Rev. C37, 1036

JOUR Nucl.Phys. A483, 406

JoUR Phys.Rev. C35, 1746

JOUR Phys.Rev. C35, 1746

JoUR Phys.Rev. C36, 1256

JOUR Astrophys.J. 324, 953

JoUR Phys.Rev. C37, 1808

JOUR Nucl.Phys. A464, 90

JOUR Z.Phys. A327, 71

JoUR Nucl.Instr.Meth. Phys.Res. A260, 33

JoUR Nucl.Phys. A467, 240

JoUR Nucl.Instr.Meth. Phys.Res. B28, 199

JoUR Nucl.Instr.Meth. Phys.Res. B28, 199

JoUR J.Radioanal.Nucl.Chem. 102, 159

JOUR Phys.Rev. C37, 2289

JOI'R Z.Phys. A327, 461

JOUR Nucl.Phys. A483, 9

JOUR Phys.Rev. C37, 1808

JouR Astro;inys.J. 316, 162

JOUR Astrop'yys.J. 316, 162

JOUR Nucl.Phys. A480, 188

JOUR Astrophys.J. 324, 953

JouR Nucl.Instr.Meth. Phys.Res. B28, 199

JoUR Nucl.Phys. A 483, 9

JoUR Phys.Rey. C35, 1214

JouR Nucl.Instr.Meth. Phys.Res. B28, 199

JOUR J.Radioanal.Nucl.Chem. 102, 159

JOUR J.Radioanal.Nucl.Chem. 102, 159

JoUR Nucl.lnstr.Meth. Phys.Res. B24/25, 722

JOUR J.Radioanal.Nucl.Chem. 102,159

JOUR Phys.Rev. C35, 1961

JoUR Astrophys.J. 324, 953

JOUR Phys.Rev. C36, 892

JOUR Phys.Rev. C35, 1961

JOUR Phys.Rev. C35, 1746

JOUR Phys.Rev. C35, 1746

JOUR Phys,Rev. C36, 1408

JOUR Nuovo Cim. 98,571

JOUR Nucl.Phys. A481, 94

JOUR Phys.Rev. C35, 1746

JouR Phys.Rev. C35, 1746

JoUR Phys.Rev.Lett. 58, 1512

JOUR Nucl.Phys. A481, 577

JOUR Nucl.Phys. A 481,577

JOUR Phys.Rev. C36, 1408

JOUR Phys.Rev. C36, 1632

JOUR Nuovo Cim. 98, 571

JOUR Phys.Scr. 36, 880

JOUR Nucl.Phys. A 481, 577

JOUR Phys.Rev. C35, 591

JOUR Phys.Rev. C36, 2341
KEY NUMBER

$86 A 104$

88 Ba 30

$88 \mathrm{Ba} 30$

87De 38

87 De 38

$88 \mathrm{Sg} 01$

$88 \mathrm{Tr} 01$

87 AT 13

B7AT13

$87 \mathrm{Ga} 15$

88 Bu01

88Le08

87Fu02

$87 \mathrm{KrO4}$

87Se 13

$87 \mathrm{Sc} 10$

$87 \operatorname{Ra} 23$

87Ra23

86 A i 04

88 i 16

87 A 505

$88 \operatorname{Cos} 0$

88Le08

B7Wi11

$87 W$ i1

88Fu02

88Bu01

87 Ra23

$88 \mathrm{Co} 10$

87La11

87 Pa23

$86 \mathrm{Al} 04$

$86 \mathrm{~A}$ i 04

87 Bo 16

$86 \mathrm{Ai} 04$

$87 \mathrm{MiO} 7$

B8Bu01

$87 \mathrm{Ha} 24$

87Mi 07

87 Ar 13

87 Ar 13

$87 \mathrm{~Pa} 24$

B7Sc33

$88 \mathrm{Ka} 13$

87Ar13

87ar13

$87 \mathrm{Ga} 03$

B8Au03

8BAu03

$87 \mathrm{~Pa} 24$

$87 \mathrm{Go} 30$

$87 \mathrm{Sc} 33$

87Sp11

88Au03

87KU02

87Mo27 


\begin{tabular}{|c|c|c|c|c|c|}
\hline TARGET & REACTION & INCIDENT ENERGY & REFE & RENCE & KEY NUMBER \\
\hline \multirow[t]{2}{*}{${ }^{17} \mathrm{O}$} & $\left({ }^{13} C_{1}{ }^{13} C^{1}\right)$ & $E \approx 10-20 \mathrm{MeV}$ & JOUR & Phys.Rev. C36, 2341 & $87 \mathrm{Mo2} 7$ \\
\hline & $\left({ }^{16} 0, x\right)$ & $\mathrm{E}(\mathrm{cm})=6-13 \mathrm{MeV}$ & JOUR & Phys.Rev. C36, 633 & $87 \mathrm{PoI1}$ \\
\hline \multirow[t]{5}{*}{${ }^{10} \mathrm{O}$} & $(p, n)$ & $\mathrm{E}<14.7 \mathrm{MeV}$ & JOUR & J.Radioanal.Nucl.Chem. 102, 159 & $86 \mathrm{~A} i 04$ \\
\hline & $(p, \alpha)$ & $E \leq 250 \mathrm{keV}$ & JOUR & 2.Phys. A327, 461 & 87 As 05 \\
\hline & $(\alpha, \gamma)$ & E not given & JOUR & Astrophys.J. 324, 953 & $88 \mathrm{Bu} 01$ \\
\hline & $\left({ }^{12} \mathrm{C},{ }^{12} \mathrm{C}^{1}\right)$ & $\mathrm{E}(\mathrm{cm}) \approx 22-32 \mathrm{MeV}$ & JOUR & Phys.Rev. C36, 2341 & $87 \mathrm{Mo} 27$ \\
\hline & $\left({ }^{16} 0, X\right)$ & $E(c m)=6-13 \mathrm{MeV}$ & JOUR & Phys.Rev. C36, 633 & 87 PoI 1 \\
\hline${ }^{17} \mathrm{~F}$ & $(p, \gamma)$ & $\mathrm{E}(\mathrm{cm}) \leq 0.7 \mathrm{MeV}$ & JOUR & Astrophys.J. 326, 384 & $88 \%$ i 08 \\
\hline \multirow[t]{5}{*}{${ }^{10} \mathrm{~F}$} & $(p, \gamma)$ & $\mathrm{E}=7-9 \mathrm{MeV}$ & JOUR & Nucl.Instr.Meth. Phys.Res. B28, 199 & $87 R a 23$ \\
\hline & $(p, \gamma)$ & $\mathrm{E}=3-10 \mathrm{MeV}$ & JOUR & Phys.Rev. C37, 2281 & $88 \mathrm{KuDB}$ \\
\hline & $(p, \gamma)$ & $E=3.5-13.3 \mathrm{MeV}$ & JOUR & Phys.Rev. C37, 2301 & $88 \mathrm{Ha} 13$ \\
\hline & $(p, n)$ & $\mathrm{E}=7-9 \mathrm{MeV}$ & JOUR & Nucl.Instr.Meth. Phys.Res, B28, 199 & $87 R a 23$ \\
\hline & $(\alpha, n)$ & $\mathrm{E} \leq 10 \mathrm{MeV}$ & JOUR & At.Energ. 62, 232 & 87Vu02 \\
\hline \multirow[t]{3}{*}{${ }^{20} \mathrm{Ne}$} & $(p, \gamma)$ & $\mathrm{E}=1165-1185 \mathrm{keV}$ & JOUR & Nucl.lnstr.Meth. Phys.Res. A260, 33 & $87 \operatorname{se} 13$ \\
\hline & $\left({ }^{12} \mathrm{C}, \mathrm{X}\right)$ & $\mathrm{E}(\mathrm{cm}) \approx 8-15 \mathrm{MeV}$ & JOUR & Nuovo Cim.98, 571 & $87 \mathrm{Sc} 33$ \\
\hline & $\left({ }^{20} \mathrm{Ne}, \mathrm{X}\right)$ & $E(c m)=15-55 \mathrm{MeV}$ & JOUR & Phys.Rev. C35, 1605 & $87 K 112$ \\
\hline \multirow[t]{5}{*}{${ }^{23} \mathrm{Na}$} & $(p, \gamma)$ & $\mathrm{E}=7-9 \mathrm{MeV}$ & JOUR & Nucl.Instr.Meth. Phys.Res. B28, 199 & $87 R a 23$ \\
\hline & $(p, \gamma)$ & $\mathrm{E}=300-400 \mathrm{keV}$ & JOUR & Nucl.lnstr.Meth. Phys.Res. A260, 33 & $87 \operatorname{Se} 13$ \\
\hline & $(p, n)$ & $E=7-9 \mathrm{MeV}$ & JOUR & Nucl.Instr.Metin. Phys.Res. B28, 199 & $87 \operatorname{Ra} 33$ \\
\hline & $(\alpha, n)$ & $E=3.9-5.9 \mathrm{MeV}$ & JOUR & Nucl.Phys. A467, 511 & 87Do06 \\
\hline & $\left({ }^{23} \mathrm{Na}, \mathrm{X}\right)$ & $E=80-177 \mathrm{MeV}$ & .JOUR & Z.Phys. A329, 103 & $88 B 101$ \\
\hline \multirow[t]{3}{*}{$M g$} & $(p, n)$ & $\mathrm{E}=6 \mathrm{MeV}$ & JOUR & Izv.Akad.Nauk SSSR, Ser.Fiz. 50, 160 & $862 a 33$ \\
\hline & $(p, p)$ & $E=6 \mathrm{MeV}$ & JOUR & Izv.Akad.Nauk SSSR, Ser.Fiz. 50, 160 & B6Za 13 \\
\hline & $(p, x \cap y p z \alpha)$ & $\mathrm{E}=\mathbf{8 . 9}-\mathbf{5 0} \mathrm{MeV}$ & JOUR & Phys.Rev. C37, 1808 & B8Le08 \\
\hline \multirow[t]{6}{*}{${ }^{24} \mathrm{Mg}$} & $(p, \gamma)$ & $\mathrm{E}=7-9 \mathrm{MeV}$ & JOUR & Nucl.1nstr.Meth. Phys.Res. B28, 199 & $87 \mathrm{Ra} 33$ \\
\hline & $(p, n)$ & $\mathrm{E}=7-9 \mathrm{MeV}$ & JOUR & Nucl.Instr.Meth. Phys.Res. B28, 199 & 87Ra23 \\
\hline & $(d, a)$ & $E=14 \mathrm{MeV}$ & JOUR & J.Radioanal.Nucl.Chem. 127. 31 & 88Dezo \\
\hline & $\left({ }^{11} B, n \alpha\right)$ & $\mathrm{E}=6-32 \mathrm{MeV}$ & JOUR & Radiochim.Acta 43, 139 & $88 \operatorname{Col} 2$ \\
\hline & $\left({ }^{10} 0, x\right)$ & $E(\mathrm{~cm})=15-55 \mathrm{MeV}$ & JOUR & Phys.Rev. C35, 1605 & $87 \mathrm{Ki} 12$ \\
\hline & $\left({ }^{32} S, X\right)$ & $E=141.8 \quad \mathrm{MeV}$ & JOUR & Phys.Rev.lett. 59,2856 & $875 a 52$ \\
\hline \multirow[t]{3}{*}{${ }^{25} \mathrm{Mg}$} & $(p, \gamma)$ & $E \approx$ resonance & JOUR & Nucl.Phys. A467, 261 & $87 \mathrm{EnO}$ \\
\hline & $(t, p)$ & $\mathrm{E}=3-12 \mathrm{MeV}$ & JOUR & Nucl.Instr.Meth. Phys.Res. B24/25. 722 & $87 \mathrm{Bo}] 6$ \\
\hline & $\left({ }^{11} \mathrm{~B}, \operatorname{2n} \alpha\right)$ & $E=6-32 \mathrm{MeV}$ & JOUR & Radjochim. Acta 43, 139 & $88 C 012$ \\
\hline \multirow[t]{7}{*}{${ }^{26} \mathrm{Mg}$} & $(p, \gamma)$ & $\mathrm{E}=7-9 \mathrm{MeV}$ & JOUR & Nucl.Instr.Meth. Phys.Res. B28, 199 & $87 R a 23$ \\
\hline & $(p, n)$ & $E=5.3-7 \mathrm{MeV}$ & JOUR & Nucl.Phys. A467, 511 & $87 D 006$ \\
\hline & $(p, n)$ & $\mathrm{E}=7-9 \mathrm{MeV}$ & JOUR & Nucl.Instr.Meth. Phys.Res. B28, 199 & 87Ra23 \\
\hline & $\left(p, p^{\prime}\right)$ & $E=5.3-6.1 \mathrm{MeV}$ & JOUR & Nucl.Phys. A467, 511 & $87 D 006$ \\
\hline & $(p, a)$ & $E=5.3-6.1 \mathrm{MeV}$ & JOUR & Nucl.Phys. A467, 511 & 87D006 \\
\hline & $(t, p)$ & $\mathrm{E}=3-12 \mathrm{MeV}$ & JOUR & Nucl.Jnstr.Meth. Phys.Res. B24/25, 722 & 878016 \\
\hline & $\left({ }^{11} \mathrm{~B}, 3 \mathrm{n} \alpha\right)$ & $\mathrm{E}=6-32 \mathrm{MeV}$ & JOUR & Radiochim.Acta 43,139 & $88 C 012$ \\
\hline${ }^{26} \mathrm{~A} 1$ & $(p, \gamma)$ & $\mathrm{E}=0.05-0.15 \mathrm{MeV}$ & JOUR & Astrophys.J. .720, 405 & $87 R a 26$ \\
\hline \multirow[t]{12}{*}{${ }^{27} \mathrm{Al}$} & $(p, \gamma)$ & $E=7-9 \mathrm{MeV}$ & JOUR & Nucl.lnstr.Meth. Phys.Res. B28, 199 & $87 \operatorname{Ra} 33$ \\
\hline & $(p, \gamma)$ & $\mathrm{E}=200-360 \mathrm{keV}$ & JOUR & Nucl.Phys. A477, 105 & B8T i 01 \\
\hline & $(p, n)$ & $\mathrm{E}=7-9 \mathrm{MeV}$ & JOUR & Nucl.Ingtr.Meth. Phys.Res. B28, 199 & B7Ra23 \\
\hline & $(p, n 3 p)$ & $E=13.3-67.5 \mathrm{MeV}$ & JOUR & Appl.Radiat.Isot. 39,41 & $88 \mathrm{LaO} 1$ \\
\hline & $(p, 2 n 3 p)$ & $E=13.3-67.5 \mathrm{MeV}$ & JOUR & Appl.Radiat.Isot. 39,41 & 88LaO1 \\
\hline & $(p .2 n 2 \alpha)$ & $\mathrm{E}=13.3-67.5 \mathrm{MeV}$ & JOUR & Appl.Radiat.Isot. 39, 41 & $88 \mathrm{LaO} 1$ \\
\hline & $(p, x \cap y p z a)$ & $\mathrm{E}=8.9-50 \mathrm{MeV}$ & JOUR & Phys.Rev. C37, 1808 & 8BLe08 \\
\hline & $(t, p)$ & $\mathrm{E}=3-12 \mathrm{MeV}$ & JOLR & Nucl.Instr.Meth. Phys.Res, B24/25, 722 & 87 B०16 \\
\hline & $\left({ }^{3} \mathrm{He}, \mathrm{p}\right)$ & $\mathrm{E}=2-20 \quad \mathrm{MeV}$ & JOLR & Rep.Prog.Phys. 50, 1171 & 8711022 \\
\hline & $\left({ }^{7} \mathrm{Li},{ }^{7} \mathrm{Be}\right)$ & $\mathrm{E}=78 \mathrm{MeV}$ & JOUR & Yad.Fiz. 45,1236 & 876105 \\
\hline & $\left({ }^{32} S, x\right)$ & $\mathrm{E}=67-\mathrm{s93} \mathrm{MeV}$ & JOUR & Phys.Rev. C37, 2233 & 88Do07 \\
\hline & $\left({ }^{35} \mathrm{Cl}, \mathrm{X}\right)$ & $\mathrm{E}(\mathrm{cm}) \approx 30-70 \mathrm{MeV}$ & JOUR & Nuovo Cim. 98, 571 & $875 c 3.3$ \\
\hline $\mathrm{Si}$ & $(p, n)$ & $\mathrm{E}=6 \mathrm{MeV}$ & JOUR & Izv.Akad.Nauk SSSR, Ser.Fuz. 50, 160 & 862 a 13 \\
\hline
\end{tabular}




\section{TARGET REACTION}

$\begin{array}{ll}\text { Si } & (p, p) \\ & (p, x n y p z \alpha) \\ { }^{20} \text { Si } \quad(p, \gamma) & (p, n) \\ & (p, p) \\ & (t, n) \\ & \left({ }^{7} L i,{ }^{7} B e\right) \\ & \left({ }^{11} \mathrm{~B}, n \alpha\right) \\ & \left({ }^{12} \mathrm{C}, n p\right) \\ & \left({ }^{12} \mathrm{C}, \mathrm{d}\right) \\ & \left({ }^{12} \mathrm{C}, \mathrm{X}\right) \\ & \left({ }^{12} \mathrm{C}, \mathrm{X}\right) \\ & \left({ }^{28} \mathrm{Si}, \mathrm{X}\right) \\ & \left({ }^{32} \mathrm{~S},{ }^{32} \mathrm{~S}\right) \\ & \left({ }^{32} \mathrm{~S},{ }^{36} \mathrm{Ar}\right)\end{array}$

${ }^{29} \mathrm{Si} \quad \begin{array}{ll}(p, \gamma) \\ & (p, n) \\ & \left({ }^{11} \mathrm{~B}, 2 n a\right) \\ { }^{30} \mathrm{Si} \quad & (p, \gamma) \\ & (p, n) \\ & \left({ }^{6} \mathrm{Li}, \mathrm{np}\right) \\ & \left({ }^{6} \mathrm{Li}, \mathrm{d}\right) \\ & \left({ }^{11} \mathrm{~B}, 3 \mathrm{na} \alpha\right.\end{array}$

${ }^{31} \mathrm{p} \quad(\mathrm{p}, \gamma)$

$(p, n)$

$S \quad(p, n)$

$(p, p)$

${ }^{32} \mathrm{~S} \quad(p, \gamma)$

(p.n)

$(p, p)$

$(\mathrm{t}, \mathrm{n})$

$\begin{array}{ll}{ }^{35} \mathrm{Cl} & (p, \gamma) \\ & (p, n) \\ & (\alpha, n) \\ & (\alpha, n) \\ & (\alpha, n \alpha)\end{array}$

$\begin{array}{cc}{ }^{30} \mathrm{AT} & (p, \gamma) \\ & \left(p, p^{\prime}\right) \\ & \\ { }^{30} \mathrm{~K} & (p, \gamma) \\ & (p, n) \\ & (\alpha, p)\end{array}$

${ }^{40} \mathrm{Ca} \quad(p, \gamma)$

$(p, \gamma)$

$(p, n)$

$\left(p, p^{\prime}\right)$

$(\alpha, X)$

$\left({ }^{18} \mathrm{~F}, \mathrm{X}\right)$

$\left({ }^{32} S, n 3 p\right)$

$\left({ }^{2} S, n 4 p\right)$

$\left({ }^{32} \operatorname{s,n} 4 \mathrm{p}\right)$

$\left({ }^{40} \mathrm{Ca}, \mathrm{X}\right)$

$\left({ }^{\circ} \mathrm{Ca}, \mathrm{X}\right)$

$\left({ }^{\circ 0} \mathrm{Ca}, X\right)$

$\left({ }^{40} \mathrm{Ca}, \mathrm{X}\right)$

$\left({ }^{\circ} \mathrm{Ca}, \mathrm{X}\right)$

\section{INCIDENT ENERGY}

$E=6 \mathrm{MeV}$

$E=8.9-50 \mathrm{MeV}$

$E=7-9 \mathrm{MeV}$

$E=7-9 \mathrm{MeV}$

$\mathrm{E} \leq 40 \mathrm{MeV}$

$\mathrm{E}=3-12 \mathrm{MeV}$

$\mathrm{E}=78 \mathrm{MeV}$

$E=6-32 \mathrm{MeV}$

$E(\mathrm{~cm})=13.8-22.1 \mathrm{MeV}$

$E(\mathrm{~cm})=13.8-22.1 \mathrm{MeV}$

$E(\mathrm{~cm})=15-55 \mathrm{MeV}$

$\mathrm{E}(\mathrm{cm})=25-60 \mathrm{MeV}$

E not given

$E=42-48.1 \mathrm{MeV}$

$\mathrm{E}=42-48.1 \mathrm{MeV}$

$t=7-9 \mathrm{MeV}$

$\mathrm{E}=7-9 \mathrm{MeV}$

$\mathrm{E}=6-32 \mathrm{MeV}$

$E=7-9 \mathrm{MeV}$

$\mathrm{E}=7-9 \mathrm{MeV}$

$\mathrm{E}(\mathrm{cm})=7.2-13.3 \mathrm{MeV}$

$E(\mathrm{~cm})=7.2-13.3 \mathrm{MeV}$

$E=6-32 \mathrm{MeV}$

$E=7-9 \mathrm{MeV}$

$E=7-9 \mathrm{MeV}$

$E=6 \mathrm{MeV}$

$\mathrm{E}=6 \mathrm{Me} \mathrm{V}$

$E=7-9 \mathrm{MeV}$

$\mathrm{E}=7-9 \mathrm{MeV}$

$E \leq 40 \mathrm{MeV}$

$\mathrm{E}=3-12 \mathrm{MeV}$

$E=7-9 \mathrm{MeV}$

$\mathrm{E}=7-9 \mathrm{MeV}$

$E=15-30 \mathrm{MeV}$

$E=t h r e s h o l d-26 \mathrm{MeV}$

$E=$ threshold $-26 \mathrm{MeV}$

$E=0.9-3.3 \mathrm{Me} \mathrm{V}$

$\mathrm{E}=2.6-3.3 \mathrm{MeV}$

$E=7-9 \mathrm{MeV}$

$E=7-9 \mathrm{MeV}$

$E=4.5-9 \mathrm{MeV}$

$E=640-3500 \mathrm{keV}$

$E=7-9 \mathrm{MeV}$

$\mathrm{E}=7-9 \mathrm{MeV}$

$E \approx 10-40 \mathrm{MeV}$

$\mathrm{E}=5-20 \mathrm{MeV}$

$\mathrm{E} \approx 25-100 \mathrm{MeV}$

$E=80-130 \mathrm{MeV}$

$E=80-120 \mathrm{MeV}$

$E \approx 100-180 \mathrm{MeV}$

E not given

E not given

$\mathrm{E}(\mathrm{cm}) \approx 62-250 \mathrm{MeV}$

$\mathrm{E}(\mathrm{cm}) \approx 50-65 \mathrm{MeV}$

$E=45-65 \mathrm{MeV}$

\section{REFERENCE}

JOUR lzv.Akad.Nauk SSSR, Ser.Fiz, 50, 160

JOUR Phys.Rev. C37, 1808

JOUR Nucl.Instr.Meth. Phys.Res, B2B, 199

JOUR Nucl.Instr.Meth. Phys.Res. B28, 199

JOUR IzY.Akad.Nauk SSSR, Ser.Fiz. 51, 24

JoUR Nucl.Instr.Meth. Phys.Res. B24/25, 722

JoUR Yad.Fiz. 45, 1236

JOUR Radiochim.Acta 43, 139

JOUR Phys.Rev. C35, 1746

JOUR Phys.Rev. C35, 1746

JOUR Phys.Rev. C35, 1605

JOUR Phys.Rev. C35, 1730

JoUR Phys.Scr, 36, 880

JOUR Nucl.Phys. A463, 731

JOUR Nucl.Phys. A463, 731

JOUR Nucl.Instr.Meth. Phys.Res. B28. 199

JOUR Nucl.Instr.Meth. Phys.Res. B28, 199

JOUR Radiochim.Acta 43, 139

JOUR Nucl.instr.Meth. Phys.Res. B28, 199

JouR Nucl.1nstr.Meth. Phys.Ras. B28, 199

JOUR Phys.Rev. C35, 1746

JOUR Phys.Rev. C35, 1746

JouR Radiochim.Acta 43, 139

JOUR Nucl.Instr.Meth. Phys.Res. B28, 199

Jour Nucl.Instr.Meth. Phys.Res. B28, 199

JOUR IzV.Akad.Nauk SSSR, Ser.Fiz. 50, 160 JoUR Jzv.Akad.Nauk SSSR, Ser.Fiz. 50, 160

JoUR Nucl.tnstr.Meth. Phys.Res. B2B, 199 JouR Nucl.lnstr.Meth. Phys.Res. B28, 199 JOUR Izv.Akad.Nauk SSSR, Ser.Fiz. 51, 24 jouR Nucl.Instr.Meth. Phys.Res. B24/25, 722

JOUR Nucl.Instr. Meth. Phys.Res. 828, 199 JOUR Nucl.instr.Meth. Phys.Res. B28, 199 JoUR Appl.Radiat.1sot. 39,97

JOUR Appl.Radial.1sol. 39, 479

JOUR A ppi.Radiat.Isot. 39,479

JOUR Nucl.Phys. A476, 316

JOUR Nucl.Phys. A476, 316

JoUn Nucl.Instr.Meth. Phys. Res. B28, 199

Jour Nucl.Instr.Meth. Phys.Res. B28, 199

Jour Nucl.Phys. A475, 548

JOUR Nucl.Phys. A466, 280

JoUR Nucl.instr.Meth. Phys.Res. B28, 199

JOUR Nucl.instr.Meth. Phys.Res. B28, 199

JouR Phys.Rev. C37, 906

JOUR Phys.Rey. [36, 966

JOUR Phys.Rev. C37, 2233

JOUR Phys.Rev. C37, 645

JOUR Phys.Rev. C37, 645

JOUR Phys.Rev. C37, 645

JOUR Phys.Scr. 36,880

JoUR Phys.Lett. 197, 507

JOUR Phys.Rev. C36, 1632

JOUR Nucl.Phys, A472, 571

JDUR Z.Phys. A326, 201
KEY NUMBER

$862 a 13$

88 Le 08

$878 \mathrm{2} 23$

$87 \mathrm{Ra} 23$

$87 \mathrm{RoO} 7$

87Bo16

B7G105

$88 \operatorname{Co}^{2} 2$

a7 Ar 13

87 Ar 13

$87 \mathrm{Kil} 2$

$87 \mathrm{ShOG}$

87Sp1 1

87B:03

87B103

B7Raz3

87Ra23

$8 \mathrm{BCol2}$

87Ra23

B7Ra23

87Ar 13

a7Ar 13

B8Col2

87 Ra23

$87 \mathrm{Ra} 23$

$86 \mathrm{Za} 13$

$86 \mathrm{Za} 13^{3}$

B7Ra23

87Raz3

87Ro07

87 Bo 16

87Ra23

$87 \mathrm{Ra} 23$

88GuO1

88Qa D

$88 Q$ a 01

88De03

88De03

87Raz3

$87 \operatorname{Ra} 23$

a7Sc 27

$872 \div 02$

$87 \mathrm{Ra} 23$

87Ra23

$88 \mathrm{BO} 07$

$870 h 08$

88Do0?

88Ra 06

88Ra 06

88Ra 06

87Spl1

B7 Sp08

$87 \mathrm{Go} 30$

87 Ag0 05

871 พ02 


\section{TARGET REACTION}

-Ca $\quad\left({ }^{18} 0, X\right)$ $\left({ }^{\circ} \mathrm{Ca}, \mathrm{X}\right)$

$\mathrm{Ti} \quad(p, n)$

$(\mathrm{p}, \mathrm{X})$

${ }^{46} \mathrm{Ti} \quad(p, \gamma)$

$(p, n)$

${ }^{47} \mathrm{Ti} \quad(\mathrm{p}, \gamma)$

$(\mathrm{p}, \mathrm{n})$

${ }^{4}{ }^{8} \mathrm{Ti} \quad(p, \gamma)$

$(p, n)$

$\left({ }^{23} \mathrm{Na}, \mathrm{X}\right)$

${ }^{51} \mathrm{~V} \quad(a, n)$

$(a, 3 n)$

$\mathrm{Cr} \quad(p, n)$

$(p, p)$

$(p, X)$

${ }^{32} \mathrm{Cr} \quad(p, \gamma)$

$(p, n)$

$(p, n)$

$(d, 2 n)$

(d.2n)

${ }^{55} \mathrm{Mn} \quad(p, \gamma)$

$(\mathrm{p}, \gamma)$

$(\mathrm{p}, \gamma)$

$(p, n)$

$(p, n)$

$(p, n)$

$\left(p, p^{\prime}\right)$

$\left(p, p^{\prime}\right)$

Fe $\quad(p, n)$

$(p, p)$

(p,xny $p z a)$

$(\mathrm{p}, \mathrm{X})$

$(a, x \cap p)$

$54 \mathrm{Fe} \quad(p, \gamma)$

$(p, \gamma)$

$(p, n)$

${ }^{50} \mathrm{Fe} \quad(p, \gamma)$

$(p, n)$

$\left({ }^{56} \mathrm{Fe}, \mathrm{X}\right)$

${ }^{38} \mathrm{Co} \quad(p, \gamma)$

$(p, n)$

$(p, n p)$

(p,2np)

$(\alpha, n)$

$(a, p)$

$(a, n 2 p)$

$(\alpha, n 2 p)$

$(a, n 3 p)$

$(a, n a)$

$(a, 3 n 2 p)$

$(a, 5 n p)$

$(a, 2 n a)$
INCIDENT ENEPGY

$E(\mathrm{~cm}) \approx 18-30 \mathrm{MeV}$

$E(\mathrm{~cm})=48-54 \mathrm{MeV}$

$E=6 \mathrm{MeV}$

$E=6 \mathrm{MeV}$

$E=40-600 \mathrm{MeV}$

$E=7-9 \mathrm{MeV}$

$E=7-9 \mathrm{MeV}$

$E=7-9 \mathrm{MeV}$

$E=7-9 \mathrm{MeV}$

$E=7-9 \mathrm{MeV}$

$E=7-9 \mathrm{MeV}$

$\mathrm{E}=48-72.5 \mathrm{MeV}$

$E=10-120 \mathrm{MeV}$

$E=10-120 \mathrm{MeV}$

$E=6 \quad M e V$

$E=6 \mathrm{MeV}$

$E=6-27 \quad \mathrm{MeV}$

$E=7-9 \quad \mathrm{MeV}$

$E=7-9 \mathrm{MeV}$

$\mathrm{E}=6-27 \mathrm{MeV}$

$\mathrm{E}=\mathrm{B}-20 \mathrm{MeV}$

$E=8-20 \mathrm{MeV}$

$E=1.3-2 \mathrm{MeV}$

$E=7-9 \mathrm{Me} \mathrm{V}$

$E=1.3-2 \mathrm{MeV}$

$E=1.3-2 \mathrm{MeV}$

$E=7-9 \mathrm{MeV}$

$E=1.3-2 \mathrm{MeV}$

$E=1.3-2 \mathrm{MeV}$

$E=1.3-2 \mathrm{MeV}$

$\mathrm{E}=6 \mathrm{MeV}$

$E=6 \mathrm{MeV}$

$E=8.9-50 \mathrm{MeV}$

$E=40-600 \mathrm{MeV}$

$E \approx 4-90 \mathrm{MeV}$

$E=3-9 \mathrm{MeV}$

$E=7-9 \mathrm{MeV}$

$E=7-9 \mathrm{MeV}$

$\mathrm{E}=7-9 \mathrm{MeV}$

$E=7-9 \mathrm{MeV}$

E not given

$E=7-9 \mathrm{MeV}$

$E=7-9 \mathrm{MeV}$

$E \approx 12.5-50 \mathrm{MeV}$

$E \approx 25-50 \mathrm{MeV}$

$E=5.07-9.8 \mathrm{MeV}$

$E=5.18-9.4 \mathrm{MeV}$

$E=60 \mathrm{MeV}$

$E=10-120 \mathrm{MeV}$

$\mathrm{E}=10-120 \mathrm{MeV}$

$E=10-120 \mathrm{MeV}$

$E=60 \mathrm{MeV}$

$E=10-120 \mathrm{MeV}$

$E=10-120 \mathrm{MeV}$

\section{REFERENCE}

KEY NUMBER

Jour Nuovo Cim. 98, 571

JOUR Nuovo Cim. 98, 571

875033

$875 \mathrm{s3}$

JOUR IZv.Akad.Nauk SSSR, Ser.Fiz. 50, 160 JOUR IZv.Akad.Nauk SSSR, Ser.Fiz. 50, 160 JouR Nucl.lnstr.Meth. Phys.Res. B29, 275

$862 a 13$

$862 a 13$

8TFil1

JouR Nucl.Instr. Meth. Phys Res. B28, 199 JouR Nucl.Instr.Meth. Phys.Res. B28, 199

JoUR Nucl.Instr.Meth. Phys.Res. B28, 199

JoUR Nucl.Instr.Meth. Phys.Res. B28, 199

Jour Nucl.Instr.Meth. Phys.Res. B28, 199 JouR Nucl.Instr.Meth. Phys.Res. B28, 199

JOUR Phys.Rev. C36, 1351

JOUR J.Phys.(London) G13, 535

JouR J.Phys.(London) G13, 535

JOUR Izv.Akad.Nauk SSSR, Ser.Fiz. 50, 160 JOUR lzv.Akad.Nauk SSSR, Ser.Fiz. 50, 160 JoUR Phys.Rev, C35, 2067

JoUR Nucl.Instr.Meth. Phys.Res. B28, 199

JouR Nucl.Instr. Meth. Phys.Res. B28, 199

JoUR Phys.Rev. C35, 2067

JOUR Phys.Rev. C35, 2067

JOUR Phys.Rev. C35, 2077

JOUR Czech.J.Phys. B3B, 150

JoUR Nucl.Instr.Meth. Phys.Res. B28, 199

JOUR Czech.J.Phys. B3B, 741

JOUR Czech.J.Phys. B38, 150

JouR Nuclinstr.Meth. Phys.Res. B28, 199

JOUR Czech.J.Phys. B3B, 741

JOUR Czech.J.Phys. B3B, 741

JouR Czech.J.Phys. B38, 150

$87 \operatorname{Ra} 23$

87Ra23

$87 \mathrm{Ra} 23$

87Ra23

$87 \operatorname{Ra} 3$

B7Ra23

87Bu 16

87Ra05

87RaO5

$862 a 13$

862 a 13

87 W05

$87 \operatorname{Ra} 23$

$87 \mathrm{Ra} 23$

$87 \mathrm{WeO5}$

$87 \mathrm{We} 05$

$87 \mathrm{MuOB}$

8 BSt 03

$87 R a 23$

885114

$88 \mathrm{StO}^{03}$

$87 R a 23$

885114

BBSt14

885103

JOUR 1zv.Akad.Nauk SSSR, Ser.Fiz. 50, 160 JOUR IzV.Akad.Nauk SSSR, Ser.Fiz. 50, 160 JOUR Phys.Rev. C37, 1808

JoUR Nucl.Instr.Meth. Phys.Res, B29, 275

JouR J.Radioanal.Nucl.Chem. 120, 49

JoUR Yad.Fiz. 45,614

JouR Nucl.Instr.Meth. Phys.Res. B28, 199

JouR Nucl.lnstr.Meth. Phys.Res. B28, 199

862 a 13

$862 a 13$

88 Le 08

$B 7 F 111$

88De0?

$875 k 03$

$87 \mathrm{Ra} 23$

87 Haz3

Jour Nucl.lnstr.Meth. Phys.Res. B28, 199

$87 R_{23}$

$87 \mathrm{Ra} 23$

87Sp11

JOUR Phys.Ser. 36, 880

B7Ra23

B7Ra 23

87मа6 1

B7Ba 61

B8Ti05

$88 \mathrm{~T}$ i 05

881501

B7Ra08

B7 Ra 08

g7 Ra 08

B81s01

87Ra 08

JOUR Pramana 30, 193

JoUR Nucl.instr.Meth. Phys.Res. B24/25, 484

JoUR Nucl.Instr.Meth. Phys.Res. B24/25, 484

87Ra08 
TARGET REACTIO:

${ }^{59} \mathrm{Co} \quad(\alpha, 4 \cap 2 p)$

$(\alpha, 6 n p)$

$(\alpha, 3 \cap a)$

$(a, 5 n 2 p)$

$(\alpha, n \geq \alpha)$

$(\alpha, 5 \cap 4 p)$

$(\alpha, 3 n 2 \alpha)$

$(\alpha, 3 \cap p 2 a)$

\begin{tabular}{|c|c|}
\hline $\mathrm{Ni}$ & $\begin{array}{l}(p, n) \\
(p, p) \\
(p, X) \\
(p, X) \\
(a, x \cap y p)\end{array}$ \\
\hline${ }^{58} \mathrm{Ni}$ & $\begin{array}{l}(p, \gamma) \\
(p, n) \\
(\alpha, p) \\
\left({ }^{7} \mathrm{Li},{ }^{7} \mathrm{Be}\right) \\
\left({ }^{28} \mathrm{Si}^{27} \mathrm{Al}\right) \\
\left({ }^{28} \mathrm{Si},{ }^{29} \mathrm{Si}\right) \\
\left({ }^{28} \mathrm{Si}, \mathrm{X}\right) \\
\left({ }^{30} \mathrm{Si}, \mathrm{X}\right) \\
\left({ }^{38} \mathrm{~S},{ }^{32} \mathrm{~S}\right) \\
\left({ }^{32} \mathrm{~S},{ }^{32} \mathrm{~S}^{\prime}\right) \\
\left(\left(^{35} \mathrm{Cl}, \mathrm{X}\right)\right. \\
\left({ }^{35} \mathrm{Cl}, \mathrm{X}\right) \\
\left(\left(^{36} \mathrm{~S},{ }^{66} \mathrm{~S}\right)\right. \\
\left({ }^{36} \mathrm{~S},{ }^{36} \mathrm{~S}\right) \\
\left({ }^{37} \mathrm{Cl}, \mathrm{X}\right) \\
\left({ }^{50} \mathrm{Ni}, \mathrm{X}\right) \\
\left({ }^{58} \mathrm{Ni}, \mathrm{X}\right) \\
\left({ }^{58} \mathrm{Ni}, \mathrm{X}\right) \\
\left({ }^{58} \mathrm{Ni}, \mathrm{X}\right) \\
\left({ }^{64} \mathrm{Ni}, \mathrm{X}\right)\end{array}$ \\
\hline
\end{tabular}

${ }^{80} \mathrm{Ni} \quad(\mathrm{p}, \gamma)$

$(p, n)$

$\left({ }^{7} \mathrm{Li},{ }^{7} \mathrm{Be}\right)$

${ }^{81} \mathrm{Ni} \quad(p, \gamma)$

$(p, n)$

$\left(p, p^{\prime}\right)$

${ }^{62} \mathrm{Ni} \quad\left({ }^{7} \mathrm{Li},{ }^{7} \mathrm{Be}\right)$

$\left({ }^{2 \mathrm{~B}} \mathrm{Si}^{2{ }^{27} \mathrm{Al}}\right)$

$\left({ }^{28} \mathrm{Si},{ }^{29} \mathrm{Si}\right)$

( ${ }^{2 \theta}$ Si X)

$\left({ }^{30} \mathrm{Si}, \mathrm{X}\right)$

$\left({ }^{35} \mathrm{CJ}, \mathrm{X}\right)$

${ }^{64} \mathrm{Ni} \quad(p, n)$

$\left({ }^{7} \mathrm{Li},{ }^{7} \mathrm{Be}\right)$

( $\left.{ }^{28} \mathrm{Si}^{22} \mathrm{Al}\right)$

$\left({ }^{28} \mathrm{Si}^{2 \theta}{ }^{28} \mathrm{Si}\right)$

$\left({ }^{28} \mathrm{Si}, \mathrm{X}\right)$

$\left({ }^{30} \mathrm{Si}, \mathrm{X}\right)$

$\left({ }^{32}{ }^{3},{ }^{35} Z\right)$

$\left({ }^{32} \mathrm{~S},{ }^{32} \mathrm{~S}\right)$

$\left.{ }^{35} \mathrm{Cl}, \mathrm{X}\right)$

$\left({ }^{36} \mathrm{~S},{ }^{36} \mathrm{~S}\right)$

$\left({ }^{3 \theta} \mathrm{S}^{30} \mathrm{~S}^{\prime}\right)$

$\left({ }^{3} \mathrm{Cl}, \mathrm{X}\right)$

$\left({ }^{84} \mathrm{Ni}, \mathrm{X}\right)$

$\left({ }^{84} \mathrm{Ni}, \mathrm{X}\right)$
INCIDENT ENERGY

$E=60 \mathrm{MeV}$

$E \approx 10-120 \mathrm{MeV}$

$E=10-120 \mathrm{MeV}$

$E=60 \mathrm{MeV}$

$E=10-120 \mathrm{MeV}$

$E=60 \mathrm{MeV}$

$E=10-120 \mathrm{MeV}$

$E=10-120 \mathrm{MeV}$

$E=6 \mathrm{MeV}$

$E=6 \mathrm{MeV}$

$E=40-600 \mathrm{MeV}$

$E=10-100 \mathrm{MeV}$

$E=25-60 \mathrm{MeV}$

$E=7-9 \mathrm{MeV}$

$E=7-9 \mathrm{MeV}$

$E=4-30 \mathrm{MeV}$

$E=78 \mathrm{MeV}$

$E(\mathrm{~cm})=47-67 \mathrm{MeV}$

$E(\mathrm{~cm})=47-67 \mathrm{MeV}$

$E(c m)=47-67 \mathrm{MeV}$

$E(\mathrm{~cm})=47-67 \mathrm{MeV}$

$E=94-112 \mathrm{MeV}$

$E=94-112 \mathrm{MeV}$

$\mathrm{E}(\mathrm{cm}) \approx 60-92 \mathrm{MeV}$

$E \approx 57-70 \mathrm{MeV}$

$E=94-112 \mathrm{MeV}$

$E \approx 94-112 \mathrm{MeV}$

$E \approx 57-70 \mathrm{MeV}$

$\mathrm{E}(\mathrm{cm}) \approx 92-108 \mathrm{MeV}$

$\mathrm{E}(\mathrm{cm}) \approx 92-110 \mathrm{MeV}$

$E=90-110 \mathrm{MeV}$

$E=186-220 \mathrm{MeV}$

$E=186-242 \mathrm{MeV}$

$E=7-9 \mathrm{MeV}$

$\mathrm{E}=7-9 \mathrm{MeV}$

$E \approx 78 \mathrm{MeV}$

$E=1.05-3.98 \mathrm{MeV}$

$E=3.08-4.66 \mathrm{MeV}$

$E=1.05-3.98 \mathrm{MeV}$

$E=78 \mathrm{MeV}$

$E(\mathrm{~cm})=47-67 \mathrm{MeV}$

$\mathrm{E}(\mathrm{cm})=47-67 \mathrm{MeV}$

$E(\mathrm{~cm})=47-67 \mathrm{MeV}$

$E(\mathrm{~cm})=4^{7}-67 \mathrm{MeV}$

$\mathrm{E}(\mathrm{cm}) \approx 58-110 \mathrm{MeV}$

$E=0.4-5.5 \mathrm{MeV}$

$E=78 \mathrm{MeV}$

$E(\mathrm{~cm})=47-67 \mathrm{MeV}$

$E(\mathrm{~cm})=47-67 \mathrm{MeV}$

$E(\mathrm{~cm})=47-67 \mathrm{MeV}$

$E(\mathrm{~cm})=47-67 \mathrm{MeV}$

$\mathrm{E}=94-112 \mathrm{MeV}$

$E=94-112 \mathrm{MeV}$

$E \approx 57-70 \mathrm{MeV}$

$\mathrm{E}=94-112 \mathrm{MeV}$

$\mathrm{E}=94-112 \mathrm{MeV}$

$\mathrm{E} \approx 57-70 \mathrm{MeV}$

$E=85-110 \mathrm{MeV}$

$\mathrm{E}(\mathrm{cm}) \approx 90-105 \mathrm{MeV}$

\section{REFERENCE}

KEY NUMBER

JOUR Pramana 30, 193

$881 \mathrm{s01}$

JouR Nucl.lnstr.Meth. Phys.Res. B24/25, 484

Jour Nucl.Instr.Meth. Phys.Res. B24/25, 484

JoUR Pramana 30, 193

JoUR Nucl.Instr.Meth. Phys.Res. B24/25, 484

JOUR Pramana 30, 193

JoUR Nucl.Instr.Meth. Phys.Res. B24/25, 484

JoUR Nucl.Instr.Meth. Phys.Res. B24/25, 484

JOUR Izv.Akad.Nauk SSSR, Ser.Fiz. 50, 160

JOUR IzV.Akád.Nauk SSSR, Ser.Fiz. 50, 160

JoUR Nucl.Instr.Meth. Phys.Res. B29, 275

JOUR At.Energ. 62, 411

Jour Nucl.Instr.Meth. Phys.Res. A263, 421

JoUR Nucl.Instr.Meth. Phys.Res. B28, 199

JoUR Nucl.Instr.Meth. Phys.Res. B28, 199

JOUR Rep.Prog.Phys. 50, 1171

JOUR Yad.Fiz. 45, 1236

JoUR Phys.Rev. C35, 597

JOUR Phys.Rev. C35, 597

JoUR Phys.Rev. C35, 597

JOUR Phys.Rev. C35, 597

JOUR Phys.Lett. 185, 15

$J-J R$ Phys.Lett. 185, 15

JoUR Nuovo $\mathrm{Cim} .98,571$

JOUR Z.Phys. A328, 355

JOUR Phys.Lett. 185, 15

JoUR Phys.Lett. 185, 15

JOUR Z.Phys. A 328, 355

JoUR Nuovo Cim. 98, 571

JOUR Nucl.Phys. A472, 571

JOUR Z.Phys. A326, 201

JoUR Phys.Lett. 206, 9

JoUR Phys.Lett. 206, 9

$87 \mathrm{RaOB}$

$87 \mathrm{Ra} 08$

88 I 01

87Ra08

$881 \leq 01$

$87 \mathrm{RaOB}$

$87 \mathrm{RaOB}$

B62a 13

862 a 13

$87 F\lfloor 11$

$87 \mathrm{~A} 129$

B8Ch0 1

87Ra23

87 Ra23

$87 \mathrm{Ho} 22$

$87 \mathrm{G} 105$

$87 \mathrm{LaD} 4$

$87 \mathrm{LaO} 4$

$87 \mathrm{LaO} 4$

$87 \mathrm{LaO} 4$

87 St 06

87St06

$875 \mathrm{s3}$

87 Sk07

$875 t 06$

B7st06

B7Sk07

B7Sc33

B7Ag05

87 I 102

$88 \operatorname{sic} 08$

$88 \mathrm{Sc} 08$

JouR Nucl.Instr.Meth. Phys.Res. B28, 199

$87 \operatorname{Ra} 23$

$87 \mathrm{Ra} 23$

87 G 105

JOUR Nucl.Phys. A480, 162

$88 \mathrm{Ti} 03$

$88 \mathrm{Ti03}$

88Ti03

JOUR Yad.Fiz. 45,1236

$87 G 105$

$871 . a 04$

$87 \mathrm{LaO} 04$

$871 . \mathrm{a} 04$

$871, \mathrm{a} 04$

$87 \mathrm{Sc} 33$

JOUR Rep.Prog.Phys. 50, 1171

JOUR Yad.Fiz. 45, 1236

JOUR Phys.Rev. C35, 597

JOUR Phys.Rev. C35, 597

JOUR Phys.Rev. C35, 597

JOUR Phys.Rev. C35, 597

JoUR Phys.Lett. 185, 15

JOUR Phys.Lett. 185, 15

JOUR Z.Phys. A 328, 355

JovR Phys.Lett. 185, 15

JOUR Phys.Lett, 185, 15

JOUR Z.Phys. A328, 355

JoUR 2.Phys. A326, 201

JoUR Nuct.Phys. A472, 571
87Ho22

$87 \mathrm{C} 105$

$87 \mathrm{LaO} 04$

$87 \mathrm{LaO} 04$

$87 \mathrm{LaO} 04$

$87 \mathrm{LaO} 04$

$87 \$ 106$

87 St 06

$87 \mathrm{Sk07}$

87St 06

87st 06

B7Sk0?

871 W02

$87 \mathrm{Ag} 05$ 


\begin{tabular}{|c|c|c|c|c|c|}
\hline \multirow{3}{*}{$\begin{array}{l}\text { TARGET } \\
\mathrm{Cu}\end{array}$} & \multirow{3}{*}{$\begin{array}{l}\text { REACTION } \\
(p, X) \\
(\alpha, X)\end{array}$} & \multirow{2}{*}{$\begin{array}{l}\text { INCIDENT ENERGY } \\
E=10-100 \mathrm{MeV}\end{array}$} & \multicolumn{2}{|c|}{ REFERENCE } & \multirow{2}{*}{$\begin{array}{c}\text { KEY NUMBER } \\
\text { 87A129 }\end{array}$} \\
\hline & & & JOUR & At.Energ. 62.411 & \\
\hline & & $\mathrm{E} \leq 40 \mathrm{MeV}$ & JOUR & Appl.Radiat.Isot. 38,499 & $87 \mathrm{Z} w 01$ \\
\hline \multirow[t]{3}{*}{${ }^{63} \mathrm{Cu}$} & $(p, \gamma)$ & $\mathrm{E}=7-\mathrm{g} \mathrm{MeV}$ & JOUR & Nucl.Instr.Meth. Phys.Res. B28, 199 & $87 R a 23$ \\
\hline & $(p, n)$ & $\mathrm{E}=7-9 \mathrm{MeV}$ & JOUR & Nucl.Instr.Meth. Phys.Res. B28, 199 & $87 \mathrm{Ra} 23$ \\
\hline & $(\alpha, x \cap y p)$ & $\mathrm{E}=\mathbf{4 0} \mathrm{MeV}$ & JOUR & J.Phys.Soc.Jpn. 56,3135 & $87 R+09$ \\
\hline${ }^{65} \mathrm{Cu}$ & $(\alpha, x \cap y p)$ & $E=40 \mathrm{MeV}$ & JOUR & J.Phys.Soc.Jpn. 56, 3135 & $878 \div 09$ \\
\hline \multirow[t]{2}{*}{$\mathrm{Zn}$} & $(p, n)$ & $\mathrm{E}=6 \mathrm{MeV}$ & JOUR & Izv.Akad.Nauk SSSR, Ser.Fiz, 50, 160 & $862 a 13$ \\
\hline & $(p, p)$ & $\mathrm{E}=6 \mathrm{MeV}$ & JOUR & Izv.Akad.Nauk SSSR, Ser.Fiz. 50, 160 & $86 \mathrm{Za} 13$ \\
\hline${ }^{62} \mathrm{Zn}$ & $(p, t)$ & $\mathrm{E}=35.52 \mathrm{MeV}$ & JOUR & Phys.Rev. C37, 556 & $88 \mathrm{CaO4}$ \\
\hline \multirow[t]{5}{*}{${ }^{64} \mathrm{Zn}$} & $(p . \gamma)$ & $\mathrm{E}=7-9 \mathrm{MeV}$ & JOUR & Nucl.Instr.Meth. Phys.Res. $\forall 28,199$ & $87 \mathrm{Ra} 23$ \\
\hline & $(p, \gamma)$ & $E=1.1-2.1 \mathrm{MeV}$ & JOUR & Nucl.Phys. A463, 605 & $87 \vee i 01$ \\
\hline & $(p, n)$ & $E=7-9 \quad \mathrm{MeV}$ & JOUR & Nucl.jnstr.Meth. Phys.Res. B28, 199 & $87 \mathrm{Ra} 23$ \\
\hline & $(p, t)$ & $E=35.52 \mathrm{MeV}$ & JOUR & Phys.Rev. C C 77,556 & $88 \mathrm{CaO} 04$ \\
\hline & $(t, p)$ & $\mathrm{E}=12 \mathrm{MeV}$ & JOUR & Phys.Rev. C37, 556 & $88 \mathrm{Ca} \mathrm{O4}$ \\
\hline \multirow[t]{2}{*}{${ }^{66} \mathrm{Zn}$} & $(p, t)$ & $\mathrm{E}=35.52 \mathrm{MeV}$ & JOUR & Phys.Rev. C 37,556 & $88 \mathrm{CaO} 4$ \\
\hline & $(t, p)$ & $\mathrm{E}=12 \mathrm{MeV}$ & JOUR & Phys.Rev. C37, 556 & $88 \mathrm{CaO}$ \\
\hline \multirow[t]{3}{*}{${ }^{68} \mathrm{Zn}$} & $(\mathbf{p}, \mathbf{n})$ & $\mathrm{E}=0.4-5.5 \mathrm{MeV}$ & JOUR & Rep.Prog.Phys. 50, 1171 & $87 H 022$ \\
\hline & $(p, t)$ & $\mathrm{E}=35,52 \mathrm{MeV}$ & JOUR & Phys.Rev, C37, 556 & $88 \mathrm{Ca} 04$ \\
\hline & $(t, p)$ & $\mathrm{E}=12 \mathrm{MeV}$ & JOUR & Phys.Rev. C37, 556 & $88 \mathrm{CaO} 4$ \\
\hline${ }^{70} \mathrm{Zn}$ & $(t, p)$ & $\mathrm{E}=12 \mathrm{MeV}$ & JOUR & Phys.Rev. C37, 556 & $88 \mathrm{CaO} 4$ \\
\hline \multirow[t]{4}{*}{${ }^{69} \mathrm{Ga}$} & $\left({ }^{3} \mathrm{He}, \mathrm{n}\right)$ & $\mathrm{E}=2-12 \mathrm{MeV}$ & JOUR & Nucl.Instr.Meth. Phys.Res. B18, 281 & $87 \mathrm{M}: 05$ \\
\hline & $\left({ }^{3} \mathrm{He}, 2 \mathrm{n}\right)$ & $\mathrm{E}=2-12 \mathrm{MeV}$ & JOUR & Nucl.Instr.Meth. Phys.Res. B18, 281 & $87 \mathrm{MiO5}$ \\
\hline & $\left({ }^{3} \mathrm{He}, \alpha\right)$ & $\mathrm{E}=2-12 \mathrm{MeV}$ & JOLR & Nucl.instr.Meth. Phys.Res. B18, 281 & $87 \mathrm{MIOS}$ \\
\hline & $\left({ }^{3} \mathrm{He}, \mathrm{n} \alpha\right)$ & $E=2-12$ MeV & JOUR & Nucl.Jnstr.Meth. Phys.Res. B18, 281 & $87 \mathrm{M} 105$ \\
\hline \multirow[t]{3}{*}{${ }^{31} \mathrm{Ga}$} & $(t, p)$ & $E=3-12 \mathrm{MeV}$ & JOUR & Nucl.Jnstr.Meth. Phys.Res. B24/25, 722 & B7Bo 16 \\
\hline & $\left({ }^{3} \mathrm{He}, 2 \mathrm{n}\right)$ & $E=2-12 \mathrm{MeV}$ & JOUR & Nucl.Jnstr.Meth. Phys.Res. B18, 281 & $87 \mathrm{MiO} 05$ \\
\hline & $\left({ }^{3} \mathrm{He}, 2 \mathrm{p}\right)$ & $E=2-12 \mathrm{MeV}$ & JOUR & Nucl.lnstr.Meth. Phys.Res. B18, 281 & $87 \mathrm{MiO} 05$ \\
\hline Ge & $\left({ }^{3} \mathrm{He}_{\mathrm{r}} \times \mathrm{n}\right)$ & $E=13-24 \mathrm{MeV}$ & JOUR & Phys.Rev. C38, 645 & $88 Q \mathrm{aO} 02$ \\
\hline \multirow[t]{5}{*}{${ }^{70} \mathrm{Ge}$} & $(p, \gamma)$ & $\mathrm{E}=7-9 \mathrm{MeV}$ & JOUR & Nucl.Instr.Meth. Phys.Res. B28, 199 & 878 a 33 \\
\hline & $(p, n)$ & $E=7-9 \mathrm{MeV}$ & JOUR & Nucl.Instr.Meth. Phys.Res, B28, 199 & $87 R a 23$ \\
\hline & $(\alpha, n)$ & $E=t h r e s h o l d-24 \mathrm{MeV}$ & JOUR & Rev.Roum.Phys. 32,725 & $87 \mathrm{Ca33}$ \\
\hline & $(\alpha, n)$ & $E=13-27 \mathrm{MeV}$ & JOUR & Phys.Rev. C38, 645 & $880 a 02$ \\
\hline & $(\alpha, 2 n)$ & $E=$ threshold $-24 \mathrm{MeV}$ & JOUR & Rev.Roum.Phys. 32, 725 & $87 \mathrm{Ca} 33$ \\
\hline \multirow[t]{2}{*}{${ }^{72} \mathrm{Ge}$} & $(p, \gamma)$ & $\mathrm{E}=7-9 \mathrm{MeV}$ & JOUR & Nucl.Instr.Meth. Phys.Res. B28, 199 & B7Pa23 \\
\hline & $(p, n)$ & $\mathrm{E}=7-9 \mathrm{MeV}$ & JOUR & Nucl.Instr.Metb. Pbys.Res. B28, 199 & $87 \mathrm{Ha} 23$ \\
\hline \multirow[t]{2}{*}{${ }^{24} \mathrm{Ge}$} & $\left({ }^{74} \mathrm{Ge}, \mathrm{X}\right)$ & $\mathrm{E}(\mathrm{cm}) \approx 110-150 \mathrm{MeV}$ & JOUR & Nucl.Phys. A472, 571 & 87 Ag 05 \\
\hline & $\left({ }^{74} \mathrm{Ge}, \mathrm{X}\right)$ & $E=105-135 \mathrm{MeV}$ & JOUR & Z.Phys. A326, 201 & $871 \% 02$ \\
\hline \multirow[t]{5}{*}{${ }^{75} \mathrm{As}$} & $(p, 3 n)$ & $E=25-55 \mathrm{MeV}$ & JOUR & Phys.Rev. C 38,645 & BBQa 02 \\
\hline & $(d .4 n)$ & $E=25-55 \mathrm{MeV}$ & JOUR & Phys.Rev. C38, 645 & $8 B Q a 02$ \\
\hline & $(t, d)$ & $E=3-12 \mathrm{MeV}$ & JOUR & Nucl.Instr.Meth. Phys.Res. B24/25, 722 & 873016 \\
\hline & $\left({ }^{3} \mathrm{He}, \mathrm{n}\right)$ & $\mathrm{E}=2-12 \mathrm{MeV}$ & JOUR & Nucl.Instr.Meth. Phys.Res. B18, 281 & $87 \mathrm{MiOS}$ \\
\hline & $\left({ }^{3} \mathrm{He}, 2 \mathrm{n}\right)$ & $\mathrm{E}=2-12 \mathrm{MeV}$ & JOUR & Nucl.Instr.Meth. Phys.Res. B18, 281 & $87 M i 05$ \\
\hline${ }^{77} \mathrm{Se}$ & $(p n)$ & $\mathrm{E} \approx 2.8-3.5 \mathrm{MeV}$ & JOUR & Yad.Fiz. 48,12 & $88 \mathrm{Fe} 07$ \\
\hline \multirow[t]{2}{*}{${ }^{80} \mathrm{Se}$} & $\left({ }^{60} \mathrm{Se}, X\right)$ & $E=125-150 \mathrm{MeV}$ & JOUR & Z.Fhys. A326, 201 & $871 \times 02$ \\
\hline & $\left({ }^{80} \operatorname{Se}, X\right)$ & $\mathrm{E}(\mathrm{cm}) \approx 130-150 \mathrm{MeV}$ & JOUR & Nucl.Phys. A472, $5 \%$ & $87 \mathrm{AgO5}$ \\
\hline${ }^{70} \mathrm{Br}$ & $(a, 2 n)$ & $\mathrm{E} \approx$ threshold $-40 \mathrm{MeV}$ & JOUR & Radiochim.Acta 41,111 & 87QaOZ \\
\hline${ }^{61} \mathrm{Br}$ & $\left({ }^{3} \mathrm{He}, 3 \mathrm{n}\right)$ & $E \approx$ threshold $-40 \mathrm{MeV}$ & JOUR & Radiochim.Acta 41.111 & $87 Q \mathrm{aOZ}$ \\
\hline
\end{tabular}




\section{TARGET REACTION}

$\mathrm{Kr}$

( ${ }^{3}$ He.xn)

$\left({ }^{3} \mathrm{He}, x_{n}\right)$

( ${ }^{3}$ He, xnp)

(a, $\left.x_{n}\right)$

$(a, x \cap p)$

\begin{tabular}{|c|c|}
\hline${ }^{\theta} \mathrm{K} \mathrm{r}$ & $(d, n a)$ \\
\hline${ }^{82} \mathrm{Kr}$ & $(p, 2 n)$ \\
\hline${ }^{85} \mathrm{Rb}$ & $\begin{array}{l}\langle p, 4 n p \\
(a, n) \\
(a, 2 n) \\
(a, 3 n) \\
(a, n \alpha)\end{array}$ \\
\hline${ }^{87} \mathrm{Rb}$ & $\left({ }^{3} \mathrm{He}, 2 \mathrm{n}\right.$ \\
\hline${ }^{87} \mathrm{Sr}$ & $(d, n)$ \\
\hline${ }^{88} \mathrm{Sr}$ & $(p, n)$ \\
\hline${ }^{89} \mathrm{Y}$ & $\begin{array}{l}(p, r) \\
(p, n) \\
(p, n) \\
(p, 2 n) \\
(p, n p) \\
(d, 2 n)\end{array}$ \\
\hline
\end{tabular}

Zr

$(p, X)$

$(p, x)$

$(d, X)$

(d. $x)$

$(a, x)$

$(a, x)$

${ }^{90} \mathrm{Zr} \quad(\mathrm{p}, \mathrm{y})$

$(p, \gamma)$

$(p, n)$

$(p, n)$

(p.p)

$\left(p, p^{\prime}\right)$

$\left({ }^{7} \mathrm{Li},{ }^{7} \mathrm{Be}\right)$

$\left({ }^{90} \mathrm{Zr}, \mathrm{X}\right)$

$\left({ }^{90} \mathrm{Zr}, \mathrm{X}\right)$

${ }^{9} \mathrm{Z}^{2} \quad(p, n)$

${ }^{82} \mathrm{Zr}_{\mathrm{r}} \quad(p, n)$

('Li, 'Be)

${ }^{84} \mathrm{Zr} \quad\left({ }^{7} \mathrm{~L}_{1}, 3 \mathrm{n}\right)$

( $\left.{ }^{7} \mathrm{Li},{ }^{7} \mathrm{Be}\right)$

${ }^{98} \mathrm{Zr} \quad(p, n)$

$\mathrm{Nb} \quad(p, x)$

$(p, X)$

$(d, X)$

$(d, X)$

$(a, x)$

$(a, X)$

$\begin{aligned}{ }^{83} \mathrm{~N} \mathrm{~b} & (p, \gamma) \\ & (p, n) \\ & (a, n)\end{aligned}$

\section{INCIDENT ENERCY}

$E \approx 10-35 \mathrm{MeV}$

$E=10-33 \mathrm{MeV}$

$E=10-33 \mathrm{MeV}$

$E=10-25 \mathrm{MeV}$

$\mathrm{E}=10-25 \mathrm{MeV}$

$\mathrm{E}=21.5 \mathrm{MeV}$

$\mathrm{E} \approx$ threshold-40 $\mathrm{MeV}$

$\mathrm{E} \approx 50-70 \mathrm{MeV}$

$E=12.8 \mathrm{MeV}$

$\mathrm{E}=20-60 \mathrm{Me} \mathrm{V}$

$E=20-60 \mathrm{MeV}$

$\mathrm{E}=20-60 \mathrm{MeV}$

$E=19.3 \mathrm{MeV}$

$E=7.3 \mathrm{MeV}$

$E=16 \mathrm{MeV}$

$E=7-9 \mathrm{MeV}$

$E=4.59-39.56 \mathrm{MeV}$

$E=7-9 \mathrm{MeV}$

$E=4.59-39.56 \mathrm{MeV}$

$\mathrm{E}=4.59-39.56 \mathrm{MeV}$

$\mathrm{E}<13.5 \mathrm{MeV}$

$E=6.1-22.4 \mathrm{MeV}$

$\mathrm{E}=11,13,21-22.6 \mathrm{MeV}$

$E=12,20-45.2 \mathrm{MeV}$

$E=6.2-22.6 \mathrm{MeV}$

$E=11,18,26,41-46 \mathrm{MeV}$

$E=12.6-45.2 \mathrm{MeV}$

$E=7-9 \mathrm{MeV}$

$E=1.9-5.7 \mathrm{MeV}$

$E=7-9 \mathrm{MeV}$

$\mathrm{E} \leq 9 \mathrm{MeV}$

$E=2-7 \quad M e V$

$E=3.9-5.7 \mathrm{MeV}$

$E=78 \mathrm{MeV}$

$\mathrm{E}(\mathrm{cm}) \approx 172-195 \mathrm{MeV}$

$E=170-195 \mathrm{MeV}$

$E \approx 12-40 \mathrm{MeV}$

$\mathrm{E} \leqq 9 \mathrm{MeV}$

$E=78 \quad \mathrm{MeV}$

$E=23-31 \mathrm{MeV}$

$E=78 \mathrm{MeV}$

E $\leq 9 \mathrm{MeV}$

$E=11,13,21-22.6 \mathrm{MeV}$

$E=6.1-22.4 \mathrm{MeV}$

$E=6.2-22.6 \mathrm{MeV}$

$\mathrm{E}=12,20-45.2 \mathrm{MeV}$

$E=11,18,26,41-46 \mathrm{MeV}$

$\mathrm{E}=12.6-45.2 \mathrm{MeV}$

$E=7-9 \mathrm{MeV}$

$\mathrm{E}=7-9 \mathrm{MeV}$

$E=10-120 \mathrm{MeV}$

\section{REFERENCE}

JOUR Appl.Radiat.lsot. 39, 135

JOUR Appl.Radiat.1sot, 39, 135

JOUR Appl.Radiat.Isot. 39, 135

JOUR Appl.Radiat.lsot. 39. 135

JOL:R A ppl.Radiat.1sot. 39,135

JOUR Appl.Radiat.1sot. 39. 709

JOUR Radiochim.Acta 41, 111

JOUR Radiochim. Acta 41, J11

JoUR J.Radioanal.Nucl.Chem. 119, 433

JOUR J.Radioanal.Nucl.Chem, 119, 303

JoUR J.Radioanal.Nucl.Chem. 119,303

JOUR J.Radioanal.Nucl.Chem, 119, 303

JOLR J.Radioanal.Nucl.Chem, 119, 433

JOUR J.Radioanal.Nucl.Chem. 119, 433

JOUR J.Radıoanal.Nucl.Chem. 119, 433

Jour Nucl.Instr.Meth. Phys.Res. B28, 199

JoUR Phys.Rev. C38, 1624

JOUR Nucl.Instr.Meth. Phys.Res. B28, 199

JoUR Phys.Rev. C38, 1624

JOUR Phys.Rev. C3B, 1624

JOUR J.Radioanal.Nucl.Chem, 127, 7

Jol R A t.Energ. 60, 332

JOUR At.Energ. 60,332

JoUR At.Energ. 60, 332

JOUR At.Energ. 60, 332

JoUR Al.Energ. 60, 332

JOUR At.Energ. 60, 332

JoUR Nucl.Instr. Meth. Phys.Res. B26, 199

JOUR Phys.Rev. C35, 1265

JouR Nucl.Instr.Meth. Phys.Res. 828, 199

JOUR Yad.Fız, 46, 28

JouR Phys.Rev. C35, 1265

JouR Phys.Rev. C35, 1265

JOUR Yad.Fiz, 45, 1236

JOUR Nucl.Phys. A472, 571

JoUR Z.Phys. A326. 201

JOUR Phys.Rev. C38. 1601

Jol'R Yad.Fiz. 46,28

JOUR Yad.Fiz. 45,1236

JOL:R Phys.Rev. C36, 2330

JOLR Yad.Fiz. 45,1236

JOUR Yad.Fiz. 46,28

JOUR At.Energ. 60,332

JoUR At.Energ. 60,332

JoUR At.Energ. 60, 332

JOUR At.Energ. 60,332

JOUR A t.Energ. $60,33 z$

JOUR At.Energ. 60, 332

JouR Nucl.lnstr.Meth. Phys.Res. B28, 199

JoUR Nucl.Instr.Meth. Phys.Res. 828, 199

JOUR J.Phys.(London) G13. 535
KEY NUMBER

$88 \mathrm{Ta} 02$

B8Ta 02

88Ta02

$88 \mathrm{Ta} 02$

88Ta02

88De24

87Qa02

87Qa02

87 Sh 30

$875 a 49$

875349

87ऽa 49

$87 \operatorname{sh} 30$

$87 \operatorname{Sh} 30$

$87 \operatorname{sh} 30$

87Ra23

$88 \mathrm{Mu} 18$

87 Ra 23

$88 \mathrm{Mu} 18$

88 Mu 8

8BDe 19

$86 K 033$

$86 \mathrm{Ko} 33$

$86 \mathrm{Ko} 33$

$86 \mathrm{Ko} 33$

$86 \mathrm{Ko} 33$

$86 \mathrm{Ko} 33$

Q7Ra23

B7LaI2

87Ra23

B?Sk06

B7 La 12

$87 \mathrm{La} 12$

$87 \mathrm{Cl} 05$

$87 \mathrm{Ag} 05$

87 I 02

8BAn11

87Sk06

87G105

878121

87G105

87Sk06

$86 \mathrm{Ko} 33$

$86 K 033$

$86 \mathrm{~K} 033$

$86 \mathrm{Ko} 33$

B6Ko33

$86 \mathrm{Ko} 33$

87Ra23

87 A 23

87 Ra 05 


\begin{tabular}{|c|c|c|c|c|c|c|}
\hline TARCET & REACTION & \multicolumn{2}{|c|}{ INCIDENT ENERGY } & \multicolumn{2}{|c|}{ REFERENCE } & KEY NUMBER \\
\hline \multirow[t]{3}{*}{${ }^{93} \mathrm{Nb}$} & $(\alpha, 2 n)$ & \multicolumn{2}{|l|}{$E=10-120 \mathrm{MeV}$} & JOUR J & J.Phys.(London) G13, 535 & $87 R a 05$ \\
\hline & $(\alpha, x \cap 2 p)$ & \multirow{2}{*}{\multicolumn{2}{|c|}{$\begin{array}{l}\mathrm{E} \leq 90 \mathrm{MeV} \\
\mathrm{E} \leq 90 \mathrm{MeV}\end{array}$}} & JOUR & J.Radioana\}.Nucl.Chem. 121, 203 & $88 \vee a 14$ \\
\hline & $(\alpha, x n \alpha)$ & & & JOUR & J.Radioanal.Nucl.Chem 121, 203 & BBVa 14 \\
\hline \multirow[t]{2}{*}{ Mo } & $(p, n)$ & \multicolumn{2}{|l|}{$E=6 \mathrm{MeV}$} & JOUR & Izv.Akad.Nauk SSSR, Ser.Fiz, 50, 160 & $86 Z a 13$ \\
\hline & $(p, p)$ & \multicolumn{2}{|l|}{$E=6 \mathrm{MeV}$} & JOUR I & Izv.Akad.Nauk SSSR, Ser.Fiz. 50, 160 & $862 a 13$ \\
\hline \multirow[t]{5}{*}{${ }^{92} \mathrm{Mo}$} & $(p, \gamma)$ & \multicolumn{2}{|l|}{$E=7-9 \mathrm{MeV}$} & JouR & Nucl.Instr.Meth. Phys.Res. B28, 199 & $87 R a 23$ \\
\hline & $(p, \gamma)$ & $\mathrm{E} \leq 9 \mathrm{MeV}$ & & JOUR & Yad.Fiz. 46,28 & 87Sk06 \\
\hline & $(p, n)$ & \multicolumn{2}{|l|}{$E=7-9 \mathrm{MeV}$} & JOUR & Nucl.Instr.Meth. Phys.Res. B28, 199 & 87Ra23 \\
\hline & $\left({ }^{32} S, X\right)$ & \multicolumn{2}{|c|}{$\mathrm{E}(\mathrm{cm}) \approx 76-92 \mathrm{MeV}$} & JOUR & Nuovo Cim. 98,571 & $87 \mathrm{Sc} 33$ \\
\hline & $\left({ }^{32} S, X\right)$ & \multicolumn{2}{|c|}{$\mathrm{E}(\mathrm{cm}) \approx 74-90 \mathrm{MeV}$} & JOUR & Nuovo Cim. 98,571 & $87 \mathrm{Sc} 33$ \\
\hline \multirow[t]{3}{*}{${ }^{84} \mathrm{Mo}$} & $(p, n)$ & \multicolumn{2}{|l|}{ Es9 MeV } & JOUR & Yad.Fiz. 46,28 & $875 k 06$ \\
\hline & $\left({ }^{32} S, X\right)$ & \multirow{2}{*}{\multicolumn{2}{|c|}{$\begin{array}{l}\mathrm{E}(\mathrm{cm}) \approx 76-92 \mathrm{MeV} \\
\mathrm{E}(\mathrm{cm}) \approx 76-92 \mathrm{MeV}\end{array}$}} & JOUR & Nuovo Cim. 98, 571 & $875 c 33$ \\
\hline & $\left({ }^{36} \mathrm{~S}, \mathrm{X}\right)$ & & & JouR & Nuava Cim. 98, 571 & $87 \mathrm{Sc} 33$ \\
\hline \multirow[t]{3}{*}{${ }^{95} \mathrm{Mo}$} & $(p, \gamma)$ & \multicolumn{2}{|l|}{$E=7-9 \mathrm{MeV}$} & JOUR & Nucl.Instr.Meth. Phys.Res. B28, 199 & 87Ra23 \\
\hline & $(p, n)$ & $\mathrm{E}=7-9 \mathrm{MeV}$ & & JOUR & Nucl.Instr.Meth. Phys.Res. B2B, 199 & $87 \mathrm{Ra} 23$ \\
\hline & $(p, n)$ & \multicolumn{2}{|l|}{$\mathrm{E} \leq 9 \mathrm{MeV}$} & JOUR & Yad.Fiz. 46,28 & 87Sk06 \\
\hline \multirow[t]{3}{*}{${ }^{96} \mathrm{Mo}$} & $(p, n)$ & \multicolumn{2}{|l|}{$\mathrm{E} \leq 9 \mathrm{MeV}$} & JOUR & Yad.Fiz. 46,28 & 875k06 \\
\hline & $\left({ }^{32} S, X\right)$ & $E(c m) \approx 74-90$ & $\mathrm{MeV}$ & JoUR & Nuovo Cim. 98, 571 & $875 c 33$ \\
\hline & $\left({ }^{36} \mathrm{~S}, \mathrm{X}\right)$ & $E(\mathrm{~cm}) \approx 76-92$ & $\mathrm{MeV}$ & JOUR & Nuovo Cim. 98, 571 & $875 c 33$ \\
\hline${ }^{98} \mathrm{Mo}$ & $\left({ }^{32} S, X\right)$ & $E(\mathrm{~cm}) \approx 74-90$ & $\mathrm{MeV}$ & JOUR & Nuovo Cim. 98, 571 & $875 c 33$ \\
\hline & $\left({ }^{36} \mathrm{~S}, \mathrm{X}\right)$ & $E(\mathrm{~cm}) \approx 76-92$ & $\mathrm{MeV}$ & JOUR & Nuava Cim. 98, 571 & $875 c 33$ \\
\hline${ }^{100} \mathrm{Mo}$ & $(p, n)$ & $\mathrm{E} \leq 9 \mathrm{MeV}$ & & JOUR & Yad.Fiz. 46,28 & $875 k 06$ \\
\hline & $\left({ }^{32} S, X\right)$ & $E(\mathrm{~cm}) \approx 76-92$ & $\mathrm{MeV}$ & JOUR & Nuova Cim. 98, 571 & $875 c 33$ \\
\hline & $\left({ }^{32} S, X\right)$ & $E(\mathrm{~cm}) \approx 72-88$ & $\mathrm{MeV}$ & JOJR & Nuovo Cim. 98, 571 & $875 \mathrm{c} 33$ \\
\hline & $\left({ }^{36} \mathrm{~S}, \mathrm{X}\right)$ & $E(\mathrm{~cm}) \approx 74-90$ & $\mathrm{MeV}$ & JOUR & Nuova Cim. 98. 571 & $87 \mathrm{Sc} 33$ \\
\hline $\mathbf{R u}$ & $(p, n)$ & $\mathrm{E}=6 \mathrm{MeV}$ & & JOUR & Izv.Akad.Nauk SSSR, Ser.Fiz. 50, 160 & 862 a 13 \\
\hline & $(p . p)$ & $\mathrm{E}=6 \mathrm{MeV}$ & & JOUR & Izv.Akad.Nauk SSSR, Ser.Fiz. 50, 160 & $862 a 13$ \\
\hline${ }^{96} \mathbf{R u}$ & $(p, \gamma)$ & $E=7-9 \mathrm{MeV}$ & & JOUR & Nucl.Instr.Meth. Phys.Res. B28, 199 & $87 \mathrm{Ra} 23$ \\
\hline & $(p, n)$ & $E=7-9 \mathrm{MeV}$ & & JOUR & Nucl.lnstr.Meth. Phys.Res. B28, 199 & $87 R a 23$ \\
\hline${ }^{100} \mathrm{Ru}$ & $\left({ }^{32} \mathrm{~S}, \mathrm{X}\right)$ & $E(\mathrm{~cm}) \approx 76-92$ & $\mathrm{MeV}$ & JOUR & Nuovo Cim. 98, 571 & $875 c 33$ \\
\hline & $\left({ }^{36} \mathrm{~S}, \mathrm{X}\right)$ & $E(c m) \approx 76-92$ & $\mathrm{MeV}$ & JOUR & Nuovo Cim. 98, 571 & $87 \mathrm{Sc} 33$ \\
\hline${ }^{101} \mathrm{Ru}$ & $\left(3^{32} S, X\right)$ & $E(\mathrm{~cm}) \approx 80-92$ & $\mathrm{MeV}$ & JouR & Nuovo Cim. 98, 571 & $87 \mathrm{Sc} 33$ \\
\hline & $\left({ }^{36} \mathrm{~S}, \mathrm{X}\right)$ & $E(c m) \approx 76-92$ & $\mathrm{MeV}$ & JOUR & Nuovo Cim. 98, 571 & $87 \mathrm{Sc} 33$ \\
\hline${ }^{102} \mathrm{Ru}$ & $\left({ }^{36} \mathrm{~S}, \mathrm{X}\right)$ & $E(c m) \approx 76-92$ & $\mathrm{MeV}$ & JOUR & Nuovo Cim. 98,571 & $87 \mathrm{Sc} 33$ \\
\hline${ }^{103} \mathrm{Ru}$ & $\left({ }^{32} \mathrm{~S}, \mathrm{X}\right)$ & $\mathrm{E}(\mathrm{cm}) \approx 76-92$ & $\mathrm{MeV}$ & JouR & Nuavo Cim. 98, 571 & $875 c 33$ \\
\hline${ }^{104} \mathrm{Ru}$ & $(p, y)$ & $\mathrm{E}=7-9 \mathrm{MeV}$ & & JOUR & Nucl.Instr.Meth. Phys.Res. B28, 199 & $87 R a 23$ \\
\hline & $(p, n)$ & $E=7-9 \mathrm{MeV}$ & & JOUR & Nucl.Instr.Meth. Phys.Res. B2B, 199 & B7Ra 23 \\
\hline & $\left({ }^{32} S, X\right)$ & $E(c m) \approx 76-92$ & $\mathrm{MeV}$ & JOUR & Nuovo Cim. 98, 571 & $87 \mathrm{Sc} 33$ \\
\hline & $\left({ }^{36} \mathrm{~S}, \mathrm{X}\right)$ & $E(\mathrm{~cm}) \approx 76-92$ & $\mathrm{MeV}$ & JOUR & Nuovo Cim. 98, 571 & $875 c 33$ \\
\hline${ }^{103} \mathrm{Rh}$ & $(p, x)$ & $E=23.3-67.1 \mathrm{M}$ & $\mathrm{MeV}$ & JoUR & Appl.Radiat.Isot. 38,151 & $87 \mathrm{La} 09$ \\
\hline & $\left({ }^{36} \mathrm{~S}, \mathrm{X}\right)$ & $E(\mathrm{~cm}) \approx 76-92$ & $\mathrm{MeV}$ & JOUR & Nuovo Cim. 98, 571 & $87 \mathrm{Sc} 33$ \\
\hline Pd & $(p, n)$ & $\mathrm{E}=6 \quad \mathrm{MeV}$ & & JOUR & Izv.Akad.Nauk SSSR, Ser.Fiz. 50, 160 & 862 a 13 \\
\hline & (p.p) & $E=6 \quad \mathrm{MeV}$ & & JOUR & „zv.Akad.Nauk SSSR. Ser.Fiz. 50, 160 & 862 a 13 \\
\hline & $(\alpha, x \cap y)$ & $E=25-60 \mathrm{MeV}$ & & Jour & Nucl.Instr-Meth. Fnys.Res. A263, 421 & $88 \mathrm{ChO} 1$ \\
\hline${ }^{104} \mathrm{Pd}$ & $(p, n)$ & $E=5-9 \mathrm{MeV}$ & & JOUR & Yad.Fiz. 47,609 & $8 \mathrm{BBa} 18$ \\
\hline & $\left({ }^{32} S, X\right)$ & $E(\mathrm{~cm}) \approx 76-92$ & $\mathrm{MeV}$ & JOUR & Nuovo Cim. 98,571 & $87 \mathrm{Sc} 33$ \\
\hline & $\left({ }^{\mathrm{J} \theta} \mathrm{S}, \mathrm{X}\right)$ & $E(\mathrm{~cm}) \approx 76-92$ & $\mathrm{MeV}$ & JOUR & Nuovo Cim. 98,571 & $875 c 33$ \\
\hline${ }^{105} \mathrm{Pd}$ & $(p, n)$ & $\mathrm{E}=5-9 \mathrm{MeV}$ & & JOUR & Yad.Fiz. 47,609 & $88 \mathrm{Ba} 18$ \\
\hline
\end{tabular}




\begin{tabular}{|c|c|c|c|c|c|c|}
\hline \multirow{3}{*}{$\frac{\text { TARGET }}{{ }^{105} \mathrm{Pd}}$} & \multirow{2}{*}{$\frac{\text { REACT ION }}{\left({ }^{32} S, X\right)}$} & \multicolumn{2}{|c|}{ INCIDENT ENERGY } & \multicolumn{2}{|c|}{ REFERENCE } & \multirow{2}{*}{$\begin{array}{c}\text { KEY NUMBER } \\
87 \mathrm{Sc} 33\end{array}$} \\
\hline & & $\mathrm{E}(\mathrm{cm}) \approx 76-92 \mathrm{Me}$ & deV & JOUR & Nuovo Cim. 98,571 & \\
\hline & $\left({ }^{36} \mathrm{~S}, \mathrm{X}\right)$ & $\mathrm{E}(\mathrm{cm}) \approx 76-92 \mathrm{Me}$ & e V & JOUR & Nuovo Cim. 98, 571 & $87 \mathrm{Sc} 33$ \\
\hline \multirow[t]{3}{*}{${ }^{106} \mathrm{Pd}$} & $(p, n)$ & $E=5-9 \mathrm{MeV}$ & & JOUR & Yad.Fiz, 47,609 & $88 \mathrm{Ba} 18$ \\
\hline & $\left({ }^{32} \mathrm{~S}, \mathrm{X}\right)$ & $\mathrm{E}(\mathrm{cm}) \approx 76-92 \mathrm{Me}$ & lev & JOUR & Nuovo Cim. 98, 571 & $87 S c 33$ \\
\hline & $\left({ }^{36} \mathrm{~S}, \mathrm{X}\right)$ & $\mathrm{E}(\mathrm{cm}) \approx 76-92 \mathrm{Me}$ & IeV & JOUR & Nuovo Cim. 98,571 & $87 \operatorname{se} 33$ \\
\hline \multirow[t]{4}{*}{${ }^{108} \mathrm{Pd}$} & $(p, n)$ & $\mathrm{E}=5-9 \mathrm{MeV}$ & & JOUR & Yad.Fiz, 47, 609 & $88 \mathrm{Ba} 18$ \\
\hline & $\left({ }^{32} \mathrm{~S}, \mathrm{X}\right)$ & $\mathrm{E}(\mathrm{cm}) \approx 78-94 \mathrm{Me}$ & $1 \mathrm{eV}$ & JOUR & Nuovo Cim. 98,571 & $87 S c 33$ \\
\hline & $\left({ }^{32} S, X\right)$ & $\mathrm{E}(\mathrm{cm}) \approx 76-92 \mathrm{Me}$ & le $\mathrm{V}$ & JOUR & Nuovo Cim. 98, 571 & $87 \operatorname{Sc} 33$ \\
\hline & $\left({ }^{36} \mathrm{~S}, \mathrm{X}\right)$ & $\mathrm{E}(\mathrm{cm}) \approx 76-92 \mathrm{Me}$ & lev & JOUR & Nuovo Cim. 98, 571 & $87 \mathrm{Se} 33$ \\
\hline \multirow[t]{2}{*}{${ }^{110} \mathrm{Pd}$} & $(p, n)$ & $\mathrm{E}=5-9 \mathrm{MeV}$ & & JOUR & Yad.Fiz. 47,609 & $88 \mathrm{Ba} 18$ \\
\hline & $\left({ }^{36} \mathrm{~S}, \mathrm{X}\right)$ & $\mathrm{E}(\mathrm{cm}) \approx 76-92 \mathrm{Me}$ & $\mathrm{deV}$ & JOUR & Nuovo Cim. 98, 571 & $87 \mathrm{Sc} 33$ \\
\hline $\mathbf{A g}$ & $(a, x)$ & $\mathrm{E} \leq 90 \mathrm{MeV}$ & & JOUR & J.Radioanal.Nucl.Chem. 121, 115 & $88 \% a \perp 1$ \\
\hline \multirow[t]{8}{*}{${ }^{107} \mathrm{Ag}$} & $(p, \gamma)$ & $E=7-9 \mathrm{MeV}$ & & JouR & Nucl.lnstr.Meth. Phys.Res. B28, 199 & $87 \mathrm{Ra} 23$ \\
\hline & $(p, n)$ & $\mathrm{E}=7-9 \mathrm{MeV}$ & & JOUR & Nucl.Instr.Meth. Phys.Res. B28, 199 & $87 \operatorname{Ra} 23$ \\
\hline & $(a, n)$ & $\mathrm{E} \leq 50 \mathrm{MeV}$ & & JOUR & Nucl.Instr.Meth. Phys.Res. B24/25, 480 & $87 \mathrm{~S}$ i 11 \\
\hline & $(a, 2 n)$ & $\mathrm{E} \leq 50 \mathrm{MeV}$ & & JOUR & Nucl.lnstr.Meth. Phys.Res. B24/25, 480 & 87 S i 11 \\
\hline & $(\alpha, n a)$ & $\mathrm{E} \leq 50 \mathrm{MeV}$ & & JOUR & Nucl.Instr.Meth. Phys.Res. B24/25, 480 & $875 i 11$ \\
\hline & $(\alpha, 2 n \alpha)$ & Es50 MeV & & JOUR & Nucl.Instr.Meth. Phys.Res. B24/25, 480 & 87S I 11 \\
\hline & $(\alpha, 4 n 2 p)$ & $\mathrm{E}=60 \mathrm{MeV}$ & & JOUR & Pramana 30,193 & $88 I \leq 01$ \\
\hline & $\left({ }^{7} \mathrm{Li},{ }^{7} \mathrm{Be}\right)$ & $\mathrm{E}=78 \mathrm{MeV}$ & & JOUR & Yad.Fiz, 45,1236 & 876105 \\
\hline \multirow[t]{8}{*}{${ }^{109} \mathrm{Ag}$} & $(p, \gamma)$ & $E=7-9 \mathrm{MeV}$ & & JOUR & Nucl.Instr.Meth. Phys.Res. B28, 199 & $87 \mathrm{Ra} 23$ \\
\hline & $(p, n)$ & $\mathbf{E}=\mathbf{7}-\mathbf{9} \mathrm{MeV}$ & & JOUR & Nucl.Instr.Meth. Phys.Res. B28, 199 & 87Ra23 \\
\hline & $(\mathbf{p}, \mathbf{n})$ & $E=9 \mathrm{MeV}$ & & JOUR & J.Radioanal.Nucl.Chem. 117, 129 & B7Pa 19 \\
\hline & $(a, 2 n)$ & $E=60 \mathrm{MeV}$ & & JOUR & Pramana 30,193 & 881501 \\
\hline & $(a, 2 n)$ & $\mathrm{E} \leq 50 \mathrm{MeV}$ & & JOUR & Nucl.Instr.Meth. Phys.Res, B24/25, 480 & $87 \mathrm{Si} 11$ \\
\hline & $(a, 3 n)$ & Es50 MeV & & JOUR & Nucl.Instr.Meth. Phys.Res. B24/25, 480 & $87 \mathrm{Si} 11$ \\
\hline & $(a, 4 n)$ & $\mathrm{E} \leq 50 \mathrm{MeV}$ & & JOUR & Nucl.Instr.Meth. Phys.Res. B24/25, 480 & $87 \mathrm{~S} 111$ \\
\hline & $\left({ }^{7} \mathrm{Li},{ }^{7} \mathrm{Be}\right)$ & $\mathrm{E}=78 \mathrm{MeV}$ & & JOUR & Yad.Fiz. 45,1236 & $87 \mathrm{GlO} 05$ \\
\hline${ }^{110} \mathrm{Cd}$ & $(p, n)$ & $E=5,2,5.6,6 \mathrm{MeV}$ & & JoUR & Nucl.Phys. A473. 471 & $87 K 515$ \\
\hline \multirow[t]{3}{*}{${ }^{111} \mathrm{Cd}$} & $(p, \gamma)$ & $E=7-9 \mathrm{MeV}$ & & JOUR & Nucl.Instr.Meth. Phys.Res. B28, 199 & $87 \operatorname{Ra23}$ \\
\hline & $(\mathbf{p}, \mathbf{n})$ & $E=9 \mathrm{MeV}$ & & JOUR & Yad.Fiz. 47,978 & $88 \mathrm{Ba} 13$ \\
\hline & $(p, n)$ & $E=7-9 \mathrm{MeV}$ & & JOUR & Nucl.lnstr.Meth. Phys.Res. B28, I99 & $87 \mathrm{Ra23}$ \\
\hline \multirow[t]{3}{*}{${ }^{114} \mathrm{Cd}$} & $(p, \gamma)$ & $\mathrm{E}=7-9 \mathrm{MeV}$ & & JOUR & Nucl.Instr.Meth. Phys.Res. B28, 199 & $87 \mathrm{Ra23}$ \\
\hline & $(p, \gamma)$ & $\mathrm{E}=3-9 \mathrm{MeV}$ & & JOUR & Yad.Fiz. 45,614 & $87 \mathrm{Sk} 03$ \\
\hline & $(p, n)$ & $E=7-9 \mathrm{MeV}$ & & JOUR & Nucl.Instr.Meth. Phys.Res. B28, 199 & $87 R a 23$ \\
\hline \multirow[t]{3}{*}{${ }^{113} \ln$} & $(p, \gamma)$ & $E=7-9 \mathrm{MeV}$ & & JOUR & Nucl.Instr.Meth. Phys.Res. B28, 199 & $87 \mathrm{Ra} 23$ \\
\hline & $(p, n)$ & $\mathrm{E}=7-9 \mathrm{MeV}$ & & JOUR & Nucl.Instr.Meth. Phys.Res. B2B, 199 & $87 R a 23$ \\
\hline & $(p, n)$ & $\mathrm{E}=\mathrm{g} \quad \mathrm{MeV}$ & & JOUR & Yad.Fiz. 47, 978 & $88 B a 13$ \\
\hline \multirow[t]{2}{*}{${ }^{115} \mathrm{In}$} & $(p, \gamma)$ & $\mathrm{E}=7-9 \mathrm{MeV}$ & & JOUR & Nucl.Instr.Meth. Phys.Res. B28, 199 & $87 \mathrm{Ra} 23$ \\
\hline & $(p, n)$ & $\mathrm{E}=7-9 \mathrm{MeV}$ & & JOUR & Nucl.instr.Meth. Phys.Res. B28, 199 & $87 R a 23$ \\
\hline${ }^{112} \mathrm{Sn}$ & $(p, \gamma)$ & $\mathrm{E}=3-9 \mathrm{MeV}$ & & JOUR & Yad.Fiz. 45,614 & $875 k 03$ \\
\hline \multirow[t]{7}{*}{${ }^{116} \mathrm{Sn}$} & $(\mathbf{p}, \gamma)$ & $E=7-9 \mathrm{MeV}$ & & JOUR & Nucl.Instr.Meth. Phys.Res. B28, 199 & $87 \mathrm{Ra} 23$ \\
\hline & $(p, n)$ & $\mathrm{E}=7-9 \mathrm{MeV}$ & & JOUR & Nucl.Jnstr.Meth. Phys.Res. B28, 199 & $87 \mathrm{Ra23}$ \\
\hline & $(\alpha, n)$ & $E=20-29 \mathrm{MeV}$ & & JOUR & lzv.Akad.Nauk SSSR, Ser.Fiz. 52, 94 & $88 B a 52$ \\
\hline & $(\alpha, n)$ & $E=16-32 \mathrm{MeV}$ & & JOUR & Izv.Akad.Nauk SSSR, Ser.Fiz. 52,94 & $88 \mathrm{Ba} 52$ \\
\hline & $(\alpha, n)$ & $E=$ threshold -32 & $\mathrm{MeV}$ & JOUR & Izv.Akad.Nauk SSSR, Ser.Fiz. 52, 94 & $88 B a 52$ \\
\hline & $(\alpha, 2 \mathrm{n})$ & $E=20-29 \mathrm{MeV}$ & & JOUR & Izv.Akad.Nauk SSSR, Ser.Fiz. 52, 94 & $88 B a 52$ \\
\hline & $(\alpha, 2 n)$ & $E=t h r e s h o l d-32$ & MeV & JOUR & Izv.Akad.Nauk SSSR, Ser.Fiz. 52,94 & 88Ba 52 \\
\hline \multirow[t]{3}{*}{${ }^{117} \mathrm{Sn}$} & $(\alpha, 2 n)$ & $E=$ th reshold -32 & MeV & JOUR & Izv.Akad.Nauk SSSR, Sег.Fjz. 52, 94 & $88 \mathrm{Ba} 52$ \\
\hline & $(a, 2 n)$ & $E=16-32 \mathrm{MeV}$ & & JOUR & Izv.Akad.Nauk SSSR, Ser.Fjz. 52,94 & $88 B a 52$ \\
\hline & $(\alpha, 3 n)$ & $E=$ threshold -32 & $\mathrm{MeV}$ & JOUR & Izv.Akad.Nauk SSSR, Seг.Fjz. 52. 94 & 88Ba 52 \\
\hline
\end{tabular}




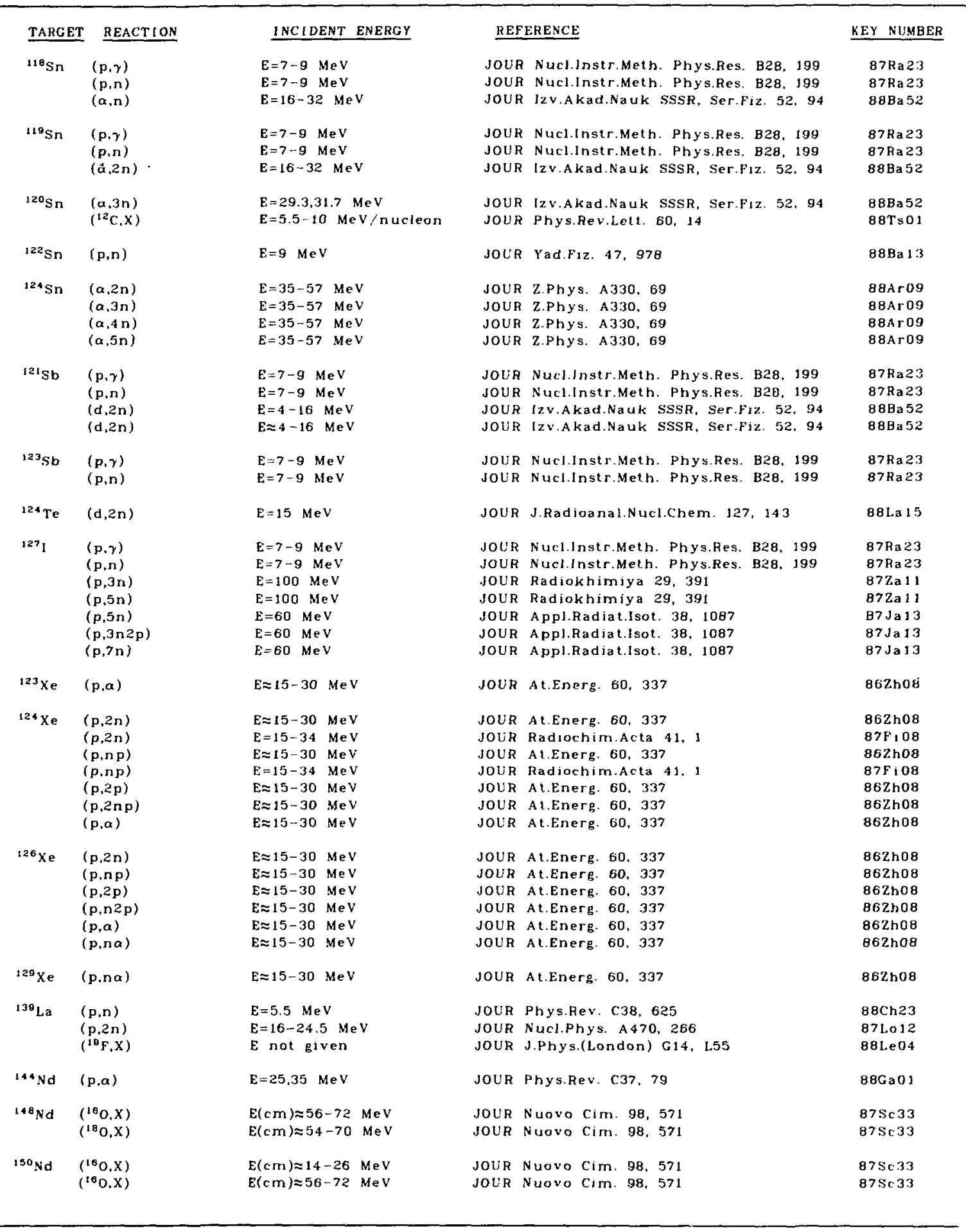




\section{TARGET REACT1ON}

$$
\begin{aligned}
& { }^{14} \mathrm{Sm} \quad\left({ }^{16} 0,1^{16} \mathrm{O}\right) \\
& \left({ }^{16} 0,{ }^{16} \mathrm{O}^{\circ}\right) \\
& \left({ }^{16} \mathrm{O}, \mathrm{X}\right) \\
& \left({ }^{10} \mathrm{Ar}, \mathrm{X}\right) \\
& { }^{14}{ }^{4} \mathrm{Sm} \quad\left({ }^{16} 0,{ }^{16} 0\right) \\
& \left({ }^{16} 0,{ }^{16} \mathrm{O}^{\prime}\right) \\
& \left({ }^{16} 0, X\right) \\
& \left({ }^{16} 0, x\right) \\
& \left({ }^{16} \mathrm{O}, \mathrm{X}\right) \\
& \left({ }^{40} \mathrm{~A} r, X\right) \\
& { }^{150} \mathrm{Sm} \quad\left({ }^{16} 0,{ }^{16} \mathrm{O}\right) \\
& \left({ }^{16} 0,{ }^{16} 0^{\circ}\right) \\
& \left({ }^{16} 0, X\right) \\
& \left({ }^{16} 0, X\right) \\
& \left({ }^{16} \mathrm{O}, \mathrm{X}\right) \\
& \left({ }^{16} \mathrm{O}, \mathrm{X}\right) \\
& { }^{152} \mathrm{Sm} \quad\left({ }^{12} \mathrm{C}, \mathrm{X}\right) \\
& \left({ }^{16} \mathrm{O}^{16} \mathrm{O}\right) \\
& \left({ }^{16} \mathrm{o}^{16} \mathrm{O}^{\prime}\right) \\
& \left({ }^{160} \mathrm{O}, \mathrm{X}\right) \\
& \left({ }^{16} 0, x\right) \\
& \left({ }^{10} 0, x\right) \\
& \left({ }^{16} \mathrm{O}, \mathrm{X}\right) \\
& { }^{154} \mathrm{Sm} \quad\left({ }^{16} \mathrm{O},{ }^{18} \mathrm{O}\right) \\
& \left({ }^{18} 0,{ }^{16} \mathrm{O}^{\prime}\right) \\
& \left({ }^{16} \mathrm{O}, \mathrm{X}\right) \\
& \left({ }^{16} 0, X\right) \\
& \left({ }^{16} \mathrm{O}, \mathrm{X}\right) \\
& \left({ }^{16} O, X\right) \\
& \left({ }^{40} \mathrm{Ar}, \mathrm{X}\right)
\end{aligned}
$$

${ }^{156} \mathrm{Gd} \quad(p, \gamma)$

$(p, n)$

${ }^{180} \mathrm{Gd} \quad\left({ }^{12} \mathrm{C}, \mathrm{X}\right)$

$\begin{array}{ll}\text { Tb } & (a, x \cap y p) \\ & \\ { }^{159} \mathrm{~Tb} & \left({ }^{7} L i, X\right) \\ & \left({ }^{14} \mathrm{~N}, a X\right) \\ & \left({ }^{19} \mathrm{~F}, X\right)\end{array}$

${ }^{165} \mathrm{Ho} \quad(a, 2 \mathrm{n})$

$(a, 4 n)$

${ }^{109} \mathrm{Tm} \quad\left({ }^{19} \mathrm{~F}, \mathrm{X}\right)$

Yb $\quad(p, x n)$

${ }^{17 \theta_{\mathrm{HI}}} \quad\left({ }^{16} \mathrm{O}, \mathrm{X}\right)$

${ }^{178} \mathrm{HS} \quad(\mathrm{p}, \gamma)$

$(\mathrm{p}, \mathrm{n})$

$(p, n)$

${ }^{17 \theta} \mathrm{HS} \quad(\mathrm{p}, \gamma)$

(p.n)

${ }^{180} \mathrm{HF} \quad(p, \gamma)$
$E=61-72.3$

$E=61-72.3$

$E=61-72.3$

$E(\mathrm{~cm}) \approx 120-140 \mathrm{MeV}$

$\mathrm{E}=7-9 \mathrm{MeV}$

$\mathrm{E}=7-9 \mathrm{MeV}$

$E=59-75 \mathrm{MeV}$

$E=59-75 \mathrm{MeV}$

$\mathrm{E}=59-75 \mathrm{MeV}$

$\mathrm{E}=57-76 \mathrm{MeV}$

$E(\mathrm{~cm}) \approx 52-68 \mathrm{MeV}$

$\mathrm{E}(\mathrm{cm}) \approx 114-146 \mathrm{MeV}$

$E=60-75 \mathrm{MeV}$

$E=60-75 \mathrm{MeV}$

$E=60-75 \mathrm{MeV}$

$E=57-76 \mathrm{MeV}$

$E(\mathrm{~cm}) \approx 52-68 \mathrm{MeV}$

$E$ not given

$\mathrm{E}(\mathrm{cm}) \approx 42-58 \mathrm{MeV}$

$E=60-75 \mathrm{MeV}$

$E=60-75 \mathrm{MeV}$

$\mathrm{E}(\mathrm{cm}) \approx 55-70 \mathrm{MeV}$

$E(\mathrm{~cm}) \approx 52-68 \mathrm{MeV}$

$E=60-75 \mathrm{MeV}$

$E$ not given

$E=60-75 \mathrm{MeV}$

$E=60-75 \mathrm{MeV}$

$E=57-76 \mathrm{MeV}$

E not given

$E(\mathrm{~cm}) \approx 52-68 \mathrm{MeV}$

$E=60-75 \mathrm{MeV}$

$E(\mathrm{~cm}) \approx 112-144 \mathrm{MeV}$

$E=7-9 \mathrm{MeV}$

$E=7-9 \quad \mathrm{MeV}$

$E=5.5-10 \mathrm{MeV} /$ nucleon

$E=25-60 \mathrm{MeV}$

$\mathrm{E}(\mathrm{cm}) \approx 18-30 \mathrm{MeV}$

$\mathrm{E}=92-309 \mathrm{MeV}$

$E$ not given

$E=10-120 \mathrm{MeV}$

$E=10-120 \mathrm{MeV}$

E not given

$E=65-100 \mathrm{MeV}$

$E=70-90 \mathrm{MeV}$

$E=7-9 \mathrm{MeV}$

$\mathrm{E}=7-9 \mathrm{MeV}$

$\mathrm{E} \leq 9.1 \mathrm{MeV}$

$E=7-9 \mathrm{MeV}$

$\mathrm{E}=7-9 \mathrm{MeV}$

$E=7-9 \mathrm{MeV}$
REFERENCE

JOUR Nucl.Phys. A471, 587

JOUR Nucl.Phys. A 471,587

JoUR Nucl.Phys. A471, 587

JOUR Nuovo Cim. 98, 571

JouR Nuc:.Instr.Meth. Phys.Res, B28, 199

Joun Nucl.lnstr.Meth. Phys.Res. B28, 199

JoUR Nucl.Phys. A471, 587

JOUR Nucl.Phys. A471, 587

JoUR Nucl.Phys. A471, 587

JOUR Z.Phys. A327, 413

JOUR Nuovo Cim. 98, 571

JoUR Nuovo Cim. 98, 571

JoUR Nucl.Phys. A471, 587

JoUR Nucl.Phys. A471, 58?

JOUR Nucl.Phys. A471, 587

JOUR Z.Phys. A327, 413

JOUR Nuovo Cim. 98, 571

JOUR J.Phys.(London) C\$4, L55

JoUR Nuovo Cim. 98, 571

JOUR Nucl.Phys. A471, 587

JOUR Nucl.Phys. A471, 5B7

JoUR Phys.Rev. C36, 1626

JOUR NuOyo Cim. 98, 571

JoUR Nucl.Phys. A471, 587

JoUR J.Phys.(London) G14, L55

JOUR Nucl.Phys. A 471, 587

JoUR Nucl.Phys. A471, 587

JOUR Z.Phys. A327, 413

JOUR J.Phys.(London) G14, L55

JOUR Nuovo Cim. 98, 571

JOUR Nucl.Phys. A471, 587

JOUR Nuovo Cim. 98, 571

JouR Nucl.Instr. Meth. Phys.Res. B28, 199

JoUR Nucl.Instr.Meth. Phys.Res. B28, 199

JOUR Phys.Rev.Lett. 60,14

JouR Nucl.Instr.Meth. Phys.Res. A263, 421

JouR Nuovo Cim. 98, 571

JOUR Nucl.Phys. A471, 635

JOUR J.Phys.(London) G14, L55

JOUR J.Phys.(London) G13. 535

JOtR J.Phys.(London) G13, 535

JOUR J.Phys.(London) G14, L55

JOUR J.Phys.(London) G14, L55

JoUR Nucl.Instr. Meth. Phys.Res, B28, 199

Jour Nucl.instr. Meth. Phys.Res. B28, 199

JOUR Izv.Akad.NaUk SSSR, Ser.Fiz, 50, 913

JoUR Nucl.Instr. Meth. Phys.Res. B2B, $199 \quad 87$ Ra23

JoUR Nucl.Instr.Meth. Phys.Res. B?8, 199

JOUR Nucl.Instr.Meth. Phys.Res. B28, 199
$87 N_{g} 05$

BuLe04

87.23

$87 R a 23$

B6Ba 84

BาRa23

$87 K+19$

$87 \mathrm{Ki} 19$

$87 \mathrm{Sc} 33$

87 Ra23

$87 K 119$

$87 K 119$

$87 \mathrm{~K}+19$

$87 \mathrm{Sc} 33$

$87 \mathrm{Sc3}$

87K 19

$87 \mathrm{Ta} 14$

$87 \mathrm{Sc33}$

$875 \mathrm{c} 33$

$87 K 119$

$87 \mathrm{Sc} 33$

$87 \mathrm{~K}$ i 19

$87 \mathrm{~K}$ j 19

87K 19

Ta 14

B7Sc 33

87K i 19

$87 \mathrm{Ra} 23$

$87 \mathrm{Ra} 3$

88Ts01

88Ch01

878251

88Le04

B7Ra05

87 Ra05

$87 R_{23}$ 


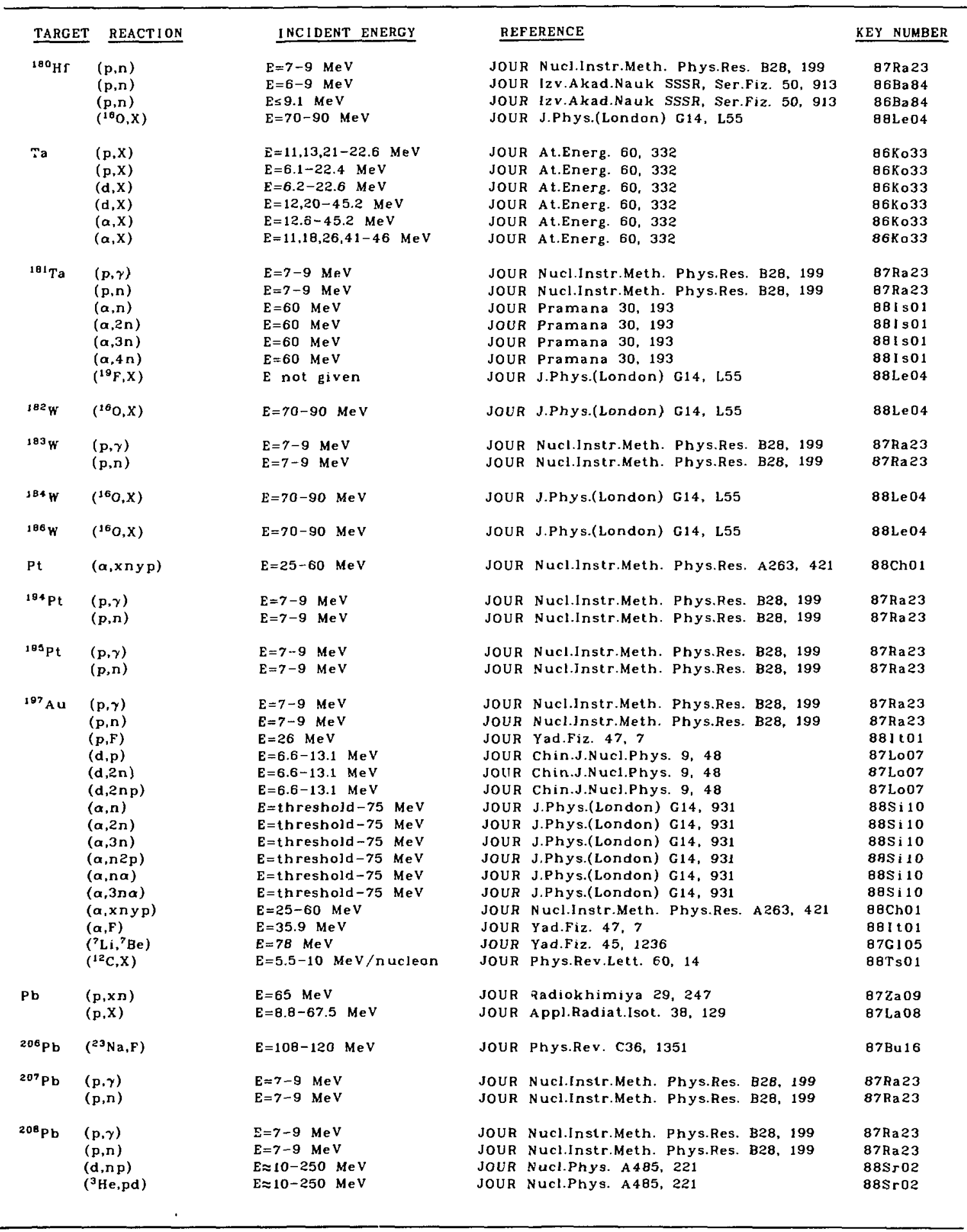




\begin{tabular}{|c|c|c|c|c|c|}
\hline TARGET & REACT ION & I NCIDENT ENERGY & REFE & RENCE & KEY NUMBER \\
\hline \multirow[t]{3}{*}{${ }^{208} \mathrm{~Pb}$} & $\left({ }^{7} \mathrm{Li},{ }^{7} \mathrm{Be}\right)$ & $E=78 \quad \mathrm{MeV}$ & JOUR & Yad.Fiz. 45,1236 & $87 G 105$ \\
\hline & $\left({ }^{1+} \mathrm{C}, 3 \mathrm{n}\right)$ & $\mathrm{E}=60-78 \mathrm{MeV}$ & JOUR & Phys.Rev. C36, 2286 & $87 \operatorname{co3} 6$ \\
\hline & $\left({ }^{16} \mathrm{O}, \mathrm{X}\right)$ & $\mathrm{E} \approx 75-135 \mathrm{MeV}$ & JOUR & Phys.Rev. C37, 998 & $88 K$ i 01 \\
\hline \multirow[t]{3}{*}{${ }^{200} \mathrm{Bi}$} & $(t, 2 n)$ & $E=11.5-17 \mathrm{MeV}$ & JOUR & Phys.Rev. C38, 74 & $88 \mathrm{Ha} 32$ \\
\hline & $(a, F)$ & $E=30.9 .31 .7 \mathrm{MeV}$ & JOUR & Yad.Fiz. 46,1380 & 871106 \\
\hline & $(a, F)$ & $E=17-31 \mathrm{MeV}$ & JOUR & Europhys.Lett. 4, 275 & $871 \mathrm{to3}$ \\
\hline \multirow[t]{3}{*}{ 232Th } & $\left({ }^{16} 0,{ }^{16} 0\right)$ & $\mathrm{E}=80 \mathrm{MeV}$ & JOUR & Phys.Rev. C35, 1600 & $87 \mathrm{Ta09}$ \\
\hline & $\left({ }^{16} \mathrm{O},{ }^{16} \mathrm{O}^{\prime}\right)$ & $\mathrm{E}=80 \mathrm{MeV}$ & soun & Phys.Rev. C35, 1600 & $87 \mathrm{TaO} 09$ \\
\hline & $\left({ }^{16} 0, x\right)$ & $\mathrm{E}=78-107 \mathrm{MeV}$ & JOUR & Z.Phys. A327, 413 & $87 \mathrm{Ta} 14$ \\
\hline${ }^{235} \mathrm{U}$ & $(\mathbf{p}, \mathbf{F})$ & $\mathrm{E}=0.5-4.3 \mathrm{MeV}$ & JOUR & Phys.Rev.lett. 58, 1520 & $87 \mathrm{Aj01}$ \\
\hline${ }^{230} \mathrm{U}$ & $\left({ }^{12} \mathrm{C}, \mathrm{X}\right)$ & $\mathrm{E}=58-85 \mathrm{MeV}$ & JoUR & Z.Phys. A327, 413 & $87 \mathrm{Ta} 14$ \\
\hline \multirow[t]{8}{*}{${ }^{238} \mathrm{U}$} & $(p, n)$ & $E \approx 10-40 \mathrm{MeV}$ & JOUR & Yad.Fiz. 46,700 & $87 \mathrm{Ag} 06$ \\
\hline & $(p, n)$ & $\mathrm{E} \approx 10-40 \mathrm{MeV}$ & JOUR & Yad.Fiz. 46,700 & $87 \mathrm{Ag} 06$ \\
\hline & $(p, 2 n)$ & $\mathrm{E} \approx 10-40 \mathrm{MeV}$ & JOUR & Yad.Fiz. 46,700 & 87Ag06 \\
\hline & $(p, 3 n)$ & $\mathrm{E} \approx 10-40 \mathrm{MeV}$ & JOUR & Yad.Fiz. 46,700 & 87 Ag06 \\
\hline & $(p, 3 n)$ & $E \approx 10-40 \mathrm{MeV}$ & JOUR & Yad.Fjz, 46, 700 & $87 \mathrm{AgO} 0$ \\
\hline & $(p, F)$ & $\mathrm{E}=0.5-4.3 \mathrm{MeV}$ & JOUR & Phys.Rev.Lett. 58,1520 & $87 \mathrm{Aj01}$ \\
\hline & $(d, \times \cap F)$ & $E \leq 20 \mathrm{MeV}$ & JOUR & Izv.Akad.Nauk SSSR, Ser.Fiz. 51, 2055 & $87 \mathrm{Gr} 33$ \\
\hline & $(\alpha, F)$ & $E=32,40,48,56 \mathrm{MeV}$ & JOUR & Nucl.Phys. A 463, 597 & $87 \mathrm{ChO} 04$ \\
\hline $2{ }^{49} \mathrm{Cf}$ & $\left({ }^{16} 0, x\right)$ & $E=90-150 \mathrm{MeV}$ & JOUR & Phys.Rev. C36, 1820 & $87 \operatorname{ch} 30$ \\
\hline \multirow[t]{3}{*}{$254 \mathrm{Es}$} & $\left({ }^{10} 0, X\right)$ & $\mathrm{E}=98 \mathrm{MeV}$ & JOUR & Rev.Roum.Phys. 32, 597 & $87 \mathrm{Ma} 50$ \\
\hline & $\left({ }^{22} \mathrm{Ne}, \mathrm{X}\right)$ & $E=126 \mathrm{MeV}$ & JOUR & Rev.Roum.Phys. 32,597 & $87 \mathrm{Ha} 50$ \\
\hline & $\left({ }^{48} \mathrm{Ca}, \mathrm{x}\right)$ & $\mathrm{E}=266 \mathrm{MeV}$ & JOUR & Rev.Roum.Phys. 32, 597 & $87 \mathrm{Ma} 50$ \\
\hline
\end{tabular}


RESIDUAL TARGET-REACTION INCIDENT ENERGY

\begin{tabular}{|c|c|}
\hline$\iota^{\prime} n$ & $\begin{array}{l}{ }^{2} \mathrm{H}\left(\mathrm{d},{ }^{3} \mathrm{He}\right) \\
{ }^{3} \mathrm{H}(\mathrm{d}, a)\end{array}$ \\
\hline${ }^{\mathrm{t}} \mathrm{H}$ & ${ }^{1} H(d, d)$ \\
\hline${ }^{2} \mathrm{H}$ & $\begin{array}{l}{ }^{2} \mathrm{H}(\mathrm{d}, \mathrm{d}) \\
{ }^{3} \mathrm{He}\left({ }^{3} \mathrm{He}, 2 \mathrm{po}\right.\end{array}$ \\
\hline${ }^{3} \mathrm{H}$ & $\begin{array}{l}{ }^{2} \mathrm{H}(\mathrm{d}, \mathrm{p}) \\
{ }^{2} \mathrm{H}(\mathrm{d}, \mathrm{p}) \\
{ }^{2} \mathrm{H}(\mathrm{d}, \mathrm{p}) \\
{ }^{3} \mathrm{H}(\mathrm{d}, \mathrm{d}) \\
{ }^{3} \mathrm{He}\left({ }^{3} \mathrm{He}, 3 \mathrm{p}\right)\end{array}$ \\
\hline${ }^{3} \mathrm{He}$ & $\begin{array}{l}{ }^{2} \mathrm{H}(\mathrm{d}, \mathrm{n}) \\
{ }^{3} \mathrm{He}\left({ }^{3} \mathrm{He}, \mathrm{n} 2\right. \\
{ }^{3} \mathrm{He}\left({ }^{3} \mathrm{He}, \mathrm{pd}\right.\end{array}$ \\
\hline${ }^{4} \mathrm{He}$ & $\begin{array}{l}{ }^{2} \mathrm{Li}(p, \alpha) \\
{ }^{2} \mathrm{H}(\mathrm{d}, \gamma) \\
{ }^{2} \mathrm{H}(\mathrm{d}, \gamma) \\
{ }^{2} \mathrm{H}(\mathrm{d}, \gamma) \\
{ }^{2} \mathrm{H}(\mathrm{d}, \gamma) \\
{ }^{2} \mathrm{H}(\mathrm{d}, \gamma) \\
{ }^{3} \mathrm{H}(\mathrm{d}, \mathrm{n}) \\
{ }^{3} \mathrm{He}(\mathrm{d}, p) \\
{ }^{3} \mathrm{He}(\mathrm{d}, p) \\
{ }^{3} \mathrm{He}(\mathrm{d}, \mathrm{p}) \\
{ }^{3} \mathrm{He}(\mathrm{d}, p) \\
{ }^{2} \mathrm{H}(\mathrm{t}, n) \\
{ }^{2} \mathrm{H}(\mathrm{t}, \mathrm{n}) \\
{ }^{2} \mathrm{H}\left({ }^{3} \mathrm{He}, p\right) \\
{ }^{3} \mathrm{He}\left({ }^{3} \mathrm{He}, 2 \mathrm{p}\right) \\
{ }^{3} \mathrm{He}\left({ }^{\mathrm{J}} \mathrm{He}, 2 \mathrm{p}\right) \\
{ }^{3} \mathrm{He}\left({ }^{3} \mathrm{He}, 2 \mathrm{p}\right)\end{array}$ \\
\hline
\end{tabular}

$\begin{array}{ll}{ }^{\gamma} \mathrm{Li} & { }^{4} \mathrm{He}(\mathrm{t}, \gamma) \\ & { }^{3} \mathrm{H}(\alpha, \gamma) \\ & { }^{3} \mathrm{H}(\alpha, \gamma) \\ & { }^{3} \mathrm{H}(\alpha, \gamma) \\ & { }^{3} \mathrm{H}(\alpha, \gamma)\end{array}$

${ }^{8} \mathbf{L i} \quad{ }^{7} \mathrm{Li}(\mathrm{t}, \mathrm{p})$

${ }^{7} \mathrm{Be} \quad{ }^{7} \mathrm{Li}(p, n)$

${ }^{3} \mathrm{He}(\alpha, \gamma)$

${ }^{3} \mathrm{He}(\alpha, \gamma)$

${ }^{8} \mathrm{Be}$

${ }^{\top} L i(p, \gamma)$

"I $B(p, a)$

${ }^{11} B(p, \alpha)$

${ }^{2} \mathrm{H}\left({ }^{11} B, \alpha\right)$

${ }^{9} \mathrm{Be} \mathrm{Li}(\mathrm{t}, \mathrm{n})$

${ }^{8} \mathrm{~B} \quad{ }^{7} \mathrm{Be}(\mathrm{p}, \gamma)$

${ }^{8} \mathrm{~B} \quad{ }^{\theta} \mathrm{Be}(\mathrm{p}, \mathrm{n})$

${ }^{10} \mathrm{~B} \quad{ }^{9} \mathrm{Be}(\mathrm{p}, \gamma)$

${ }^{13} \mathrm{C}(p, \alpha)$

${ }^{8} \operatorname{Li}\left({ }^{8} L i, n p\right)$

${ }^{B} \mathrm{Li}\left({ }^{8} \mathrm{Li}, \mathrm{d}\right)$

${ }^{11} \mathrm{~B}$
$\mathrm{E}=20-117 \mathrm{keV}$

$\mathrm{E}=80-116 \mathrm{keV}$

Es $20 \mathrm{keV}$

$E \leq 20 \mathrm{keV}$

$E=17.9,21.7,24 \mathrm{MeV}$

$E=20-117 \mathrm{keV}$

$E(\mathrm{~cm})=6.95-141.8 \mathrm{keV}$

Es250 keV

$\mathrm{E} \leqslant 20 \mathrm{keV}$

$E=17.9,21.7,24 \mathrm{MeV}$

$\mathrm{E}(\mathrm{cm})=2.98-162.5 \mathrm{keV}$

$E=17.9,21.7 .24 \mathrm{MeV}$

$E=17,9,21.7,24 \mathrm{MeV}$

Es $50 \mathrm{keV}$

$E=0.075-3 \mathrm{MeV}$

$\mathrm{E}(\mathrm{cm})=50-500 \mathrm{keV}$

$E(\mathrm{~cm})=0.010-10 \mathrm{MeV}$

$E(\mathrm{~cm})=0.010-10 \mathrm{MeV}$

$\mathrm{E}=0.001-1 \mathrm{MeV}$

$E(\mathrm{~cm})=2.98-162.5 \mathrm{keV}$

$\mathrm{E}=1 \mathrm{keV}-10 \mathrm{MeV}$

Es $250 \mathrm{keV}$

$E=0-300 \mathrm{keV}$

$E=t h$ reshold $-0.6 \mathrm{MeV}$

$E=$ threshold-0.6 MeV

$E=17.9,21,7,24 \mathrm{MeV}$

$E \leq 250 \mathrm{keV}$

$E(\mathrm{~cm})=17.9-342.5 \mathrm{keV}$

$\mathrm{E}(\mathrm{cm}) \approx 0-1.5 \mathrm{MeV}$

$E=10$ w

$E=0.7-2 \mathrm{MeV}$

$E \approx 50-600 \mathrm{keV}$

$E(\mathrm{~cm})=7.9-464 \mathrm{keV}$

$E \approx 5.7-8.5 \mathrm{MeV}$

$E=7-9 \mathrm{MeV}$

$E(\mathrm{~cm})=195-686 \mathrm{keV}$

$E=10 w$

$E=7-9 \mathrm{MeV}$

$E=140-1100 \mathrm{keV}$

$\mathrm{E} \leq 50 \mathrm{keV}$

$E=1.68-13.2 \mathrm{MeV}$

$E \approx 5.7-8.5 \mathrm{MeV}$

$\mathrm{E} \approx 2 \mathrm{MeV}$

$E=7-9 \mathrm{MeV}$

$\mathrm{E}=7-9 \mathrm{MeV}$

$\mathrm{E}=30.95 \mathrm{MeV}$

$E(\mathrm{~cm})=7.2-13.3 \mathrm{MeV}$

$E(\mathrm{~cm})=7.4,9.4 \mathrm{MeV}$

$E=19.6-25 \mathrm{MeV}$

$E=19.6-25 \mathrm{MeV}$
$E(\mathrm{~cm})=5.9-41.6 \mathrm{keV}$

\section{REFERENCE}

KEY NUMBER

JouR Nucl.Instr.Meth. Phys.Res. B10/11, 405

JOUR Phys.Rev. C35, 1999

JOUR Phys.Lett. 202, 289

g8Be06

JOUR Phys.Lett. 202, 289

JoUR Phys.Rev. C35, 383

$88 \mathrm{Be} 06$

$87 \mathrm{~B}$ г02

Jour Nucl.Instr. Meth. Phys.Res. B10/11, 405 85Jal6 JOUR Nucl.Phys. A465, 150

JOUR Z.Phys. A327, 461

JOUR Phys.Lett. 202, 289

JoUR Phys.Rev. C35, 383

JouR Nucl.Phys. A465, 150

JOUR Phys.Rev. C35. 383

JouR Phys.Rev. C35, 383

JOUR Z.Phys, A327, 461

JoUR Phys.Rev. C36, 875

JouR Phys.lett. 197, 315

JOUR Phys.Lett. 200, 246

JouR Phys.Rev. C36, 21

JOUR Phys.Rev, C36, 17

JOUR At.Energ. 63. 252

JOUR Nucl.Phys. A465, 150

JOUR Phys.lett. 202, 179

JOUR A t.Energ. 63, 252

JOUR Z.Phys. A 327, 461

JOUR Yad.Fiz. 46, 757

JoUR Yad.Fiz. 47, 1238

JOUR Yad.Fiz. 47, 1238

JOUR Phys.Rev. C 35,383

JOUR Z.Phys. A327, 46 !

Jour Nucl.Phys. A467, 273

$87 K r 18$

$87 \mathrm{~A} \leq 05$

$88 \mathrm{Be} 06$

$87 \mathrm{Br} 02$

$87 \mathrm{Kr} 18$

87日r02

$87 \mathrm{Br} 02$

$87 \mathrm{~A}=05$

$87 \mathrm{P}_{108} 08$

$87 \mathrm{Ba} 58$

$88 \mathrm{Ha} 02$

$87 \mathrm{BJ} 12$

$87 \mathrm{As} 03$

$87 \mathrm{Cu} 25$

$87 \mathrm{~K}+18$

$88 \mathrm{En} 03$

$87 \mathrm{Cu} 25$

87 As05

87 Va36

$88 \mathrm{Cu} 07$

$86 \mathrm{Gu07}$

$87 \mathrm{Br} 02$

B7ASO5

87 Kro9

JOUR Phys.Rev. C37, 512

JOUR Phys.lett. 202, 475

JoUR Nucl.Phys. A473, 179

JOUR Z.Phys. A330, 277

JouR Phys.lett. 142, 55

$88 \mathrm{Ka} 02$

$88 \mathrm{KaO} 7$

$87 \mathrm{Bu} 18$

88 A 124

$87 \mathrm{Sc} 18$

JOUR Yad.Fiz. 46, 499

87Ab 15

JOUR Nucl.Instr.Meth. Phys.Res. B28, 199 JOUR Z.Phys. A329, 243

JOUR Phys.lett. 202, 475

$87 \mathrm{Ra} 23$

$88 \mathrm{Hi} 06$

$88 \mathrm{KaO}$

Jour Nucl.Instr.Meth. Phys.Res. B28, $199 \quad 87$ Raz3

JOUR Z.Phys. A327, 341

JouR Z.Phys. A 327,461

JOUR Z.Phys. A327, 341

$87 \mathrm{Be} 17$

B7As05

$87 \mathrm{Be} 17$

JOUR Yad.Fiz. 46, 499

$87 \mathrm{Ab} 15$

JOUR Phys.Rev. C37, 2920

$88 \mathrm{Ba} 29$

JaUR Nucl.lnstr.Meth. Phys.Res. B28. 199

$87 \mathrm{Ha} 23$

JOUR Nucl.Instr.Meth. Phys.Res. B28, 199

JOUR Phys.Rev. C38, 517

JoUR Phys.Rev. C35, 1746

JOUR Phys.Rev. C35, 1746

JoUR Nuovo Cim. 97, 29

JOUR Nuovo Cim. 97. 29
$87 \mathrm{Ra}^{2} 3$

88Ba 30

87 A 13

$87 \mathrm{~A} 1 \mathrm{~B}$

$87 \mathrm{Ha} 14$

87Ra 14 


\begin{tabular}{|c|c|c|c|c|c|}
\hline RESIDUAL & TARGET-REACTION & INCIDENT ENERGY & REFE & RENCE & KEY NUHBER \\
\hline \multirow[t]{3}{*}{${ }^{11} \mathrm{~B}$} & ${ }^{10} \mathrm{He}\left({ }^{3} \mathrm{He}, \mathrm{np}\right)$ & $E=2-1 \mathrm{IS} \mathrm{MV}$ & JOUR & Nucl.Instr.Meth. Phys.Res. B18, 281 & $87 H_{1} 05$ \\
\hline & ${ }^{10} \mathrm{He}\left({ }^{3} \mathrm{He}, \mathrm{d}\right)$ & $E=2-12 \mathrm{MeV}$ & JOUR & Nucl.lnstr.Meth. Phys.Res. B18, 281 & $87 \mathrm{M}$ i 05 \\
\hline & ${ }^{\theta} \mathrm{Li}\left({ }^{\theta} \mathrm{Li}, \mathrm{p}\right)$ & $E=2-16 \mathrm{MeV}$ & JOUR & Nucl.Phys. A467, 149 & 870005 \\
\hline${ }^{10} \mathrm{C}$ & ${ }^{10} \mathrm{~B}(\mathrm{p}, \mathrm{n})$ & $\mathrm{E}=7-9 \mathrm{MeV}$ & JOUR & Nucl.Instr.Meth. Phys.Res. B28, 199 & $87 R \div 23$ \\
\hline \multirow[t]{7}{*}{ t:C } & ${ }^{10} \mathrm{~B}(\mathrm{p}, \gamma)$ & $E=7-9 \quad \mathrm{MeV}$ & JOUR & Nucl.Instr. Meth. Phys.Res. B28. 199 & $87 \mathrm{R}$ a 23 \\
\hline & ${ }^{11} \mathrm{~B}(\mathrm{p}, \mathrm{n})$ & $\mathrm{E}<14.7 \mathrm{MeV}$ & JOUR & J.Radioanal. Nucl.Chem. 102,159 & $86 \mathrm{~A} ; 04$ \\
\hline & ${ }^{11} \mathrm{~B}(\mathrm{p}, \mathrm{n})$ & $E=7-9 \mathrm{MeV}$ & JOUR & Nucl.Instr.Meth. Phys.Res. B28, 199 & $87 \mathrm{Ra} 23$ \\
\hline & $14 \mathrm{~N}(p, a)$ & $\mathrm{E}<14.7 \mathrm{MeV}$ & JOUR & J.Radioanal.Nucl.Chem. 102. 159 & $86 A, 04$ \\
\hline & ${ }^{10} \mathrm{~B}(\mathrm{t}, 2 \mathrm{2n})$ & $E=3-12 \mathrm{MeV}$ & JOUR & Nucl.Instr.Meth. Phys.Res. B24/25, 722 & $87 \mathrm{Bo} 16$ \\
\hline & ${ }^{7} \mathrm{Be}(\alpha, \gamma)$ & E not given & JOUR & Astrophys.J. 324,953 & $88 B u 01$ \\
\hline & ${ }^{6} \mathrm{Li}\left({ }^{6} \mathrm{Li}, \mathrm{n}\right)$ & $\mathrm{E}=2-16 \mathrm{MeV}$ & JOUR & Nucl.Phys. A467, 149 & 87 Do05 \\
\hline \multirow[t]{15}{*}{${ }^{12} \mathrm{C}$} & ${ }^{11} \mathrm{~B}(\mathrm{p}, \gamma)$ & $\mathrm{E}=7-9 \mathrm{MeV}$ & JOUR & Nuch.Jnstr.Meth. Phys.Res. B28, 199 & $87 \mathrm{Ra} 23$ \\
\hline & ${ }^{15} \mathrm{~N}(p, \alpha)$ & $E \leq 50 \mathrm{keV}$ & JOUR & Z.Phys. A 327, 461 & 87 As 05 \\
\hline & ${ }^{8} \mathrm{Be}(\alpha, \gamma)$ & $E(\mathrm{~cm})=0.0-4 \mathrm{MeV}$ & JOUR & Phys.Rev. C 36.54 & 87De 13 \\
\hline & $\operatorname{Li}(\alpha, n)$ & $\mathrm{E} \leq 10 \mathrm{MeV}$ & JOUR & At.Energ. 62,232 & 87 Vuoz \\
\hline & ${ }^{\theta} \mathrm{Be}(\alpha, n)$ & $\mathrm{E} \leq 10 \mathrm{MeV}$ & JOUA & AL.Energ. 62, 232 & 87 Vu02 \\
\hline & $B(\alpha, n)$ & $E \leq 10 \mathrm{MeV}$ & JOUR & At.Energ. 62, 232 & 87 Vu02 \\
\hline & $c(a, n)$ & $E \leq 10 \mathrm{MeV}$ & JOUR & A t.Energ. 62, 232 & 87 Vu02 \\
\hline & ${ }^{19} \mathrm{~F}(\alpha, n)$ & $E \leq 10 \mathrm{MeV}$ & JOUR & At.Energ. 62,232 & $87 \vee u 02$ \\
\hline & ${ }^{12} \mathrm{C}(\alpha, \alpha)$ & $E=1-66 \mathrm{MeV}$ & JOUR & Nucl.Phys. A465, 291 & $87 P 103$ \\
\hline & ${ }^{12} \mathrm{C}(a, a)$ & $\mathrm{E}(\mathrm{cm}) \approx 5-2000 \mathrm{MeV} / \mathrm{nucl}$ & JOUR & Nucl.Phys. A477, 713 & $88 \mathrm{Ma} 10$ \\
\hline & ${ }^{12} \mathrm{C}\left({ }^{12} \mathrm{C} \cdot{ }^{12} \mathrm{C}\right)$ & $E(\mathrm{~cm})=26.5-30.5 \mathrm{MeV}$ & JOUR & Phys.Rey. C37, 1953 & $88 \mathrm{~Pa} 04$ \\
\hline & ${ }^{12} \mathrm{C}\left({ }^{12} \mathrm{C},{ }^{12} \mathrm{C}\right)$ & $\mathrm{E}(\mathrm{cm}) \approx 5-2000 \mathrm{MeV} / \mathrm{nucl}$ & JOUR & Nucl.Phys. A477, 713 & $88 \mathrm{Ma} 10$ \\
\hline & ${ }^{12} \mathrm{C}\left({ }^{12} \mathrm{C} \cdot{ }^{12} \mathrm{C}\right)$ & $E(\mathrm{~cm}) \leq 14 \mathrm{MeV}$ & JOUR & Phys.Rev. C36, 1408 & $87 \mathrm{~Pa} 24$ \\
\hline & ${ }^{12} \mathrm{C}\left({ }^{15} \mathrm{~N},{ }^{15} \mathrm{~N}\right)$ & $E=51-62 \mathrm{MeV}$ & JOUR & Fizika(Zagreb) 19, Supplement 113 & $87 \mathrm{~F} \times 11$ \\
\hline & ${ }^{12} \mathrm{C}\left({ }^{15} \mathrm{~N},{ }^{13} \mathrm{~N}\right)$ & $E=51-62 \mathrm{MeV}$ & JOUR & Fizika(Zagreb) 19, Supplement 113 & $87 \mathrm{~F} \times 11$ \\
\hline \multirow[t]{2}{*}{${ }^{13} \mathrm{C}$} & ${ }^{13} \mathrm{C}(\mathrm{p}, \mathrm{p})$ & $E=30.95 \mathrm{MeV}$ & JOUA & Phys.Rev. C 38,517 & $88 \mathrm{Ba} 30$ \\
\hline & ${ }^{13} \mathrm{C}(\alpha, \alpha)$ & $E=10 w$ & JOUR & Phys.Rev. C36, 2206 & $87 \operatorname{De} 38$ \\
\hline${ }^{1 *} \mathrm{C}$ & ${ }^{11} \mathrm{~B}(\alpha, p)$ & $\mathbf{E}=0.9-1.7 \mathrm{MeV}$ & JOUR & Nucl.Phys. A468, 29 & $87 \mathrm{TuOH}$ \\
\hline \multirow[t]{6}{*}{${ }^{13} \mathrm{~N}$} & ${ }^{12} \mathrm{C}(\mathrm{p}, \gamma)$ & $E=2.65-2.85 \mathrm{MeV}$ & JouR & Nucl.instr.Meth. Phys.Res. A260, 33 & $87 \mathrm{Se} 13$ \\
\hline & ${ }^{12} \mathrm{C}(\mathrm{p}, \gamma)$ & $E=40,65,85 \mathrm{MeV}$ & JOUR & Phys.Rev. C 35,1214 & $87 \mathrm{La} 11$ \\
\hline & ${ }^{12} \mathrm{C}(\mathrm{p} . \gamma)$ & $\mathrm{E}<14.7 \mathrm{MeV}$ & JOUR & J.Radioanal.Nucl.Chem. 102, 159 & $86 A+04$ \\
\hline & ${ }^{13} \mathrm{C}(\mathrm{p}, \mathrm{n})$ & $\mathrm{E}<34.7 \mathrm{MeV}$ & JOUR & J.Radioanal.Nucl.Chem. 102. 159 & $86 \mathrm{AiO} 04$ \\
\hline & ${ }^{16} \mathrm{O}(p, \alpha)$ & $\mathrm{E}<14.7 \mathrm{MeV}$ & JOUR & J.Radioanal.Nucl.Chem. 102, 159 & $86 \mathrm{~A}$ i 04 \\
\hline & ${ }^{12} \mathrm{C}(\mathrm{d}, \mathrm{n})$ & $\mathrm{E}<14.7 \mathrm{MeV}$ & JOUR & J.Radioanal.Nucl.Chem. 102,159 & $86 \mathrm{~A} i 04$ \\
\hline${ }^{13} \mathrm{~N}$ & ${ }^{18} O(p, \alpha)$ & $E \leq 50 \mathrm{keV}$ & JOUR & Z.Phys. A327, 461 & E/As05 \\
\hline \multirow[t]{3}{*}{$16 \mathrm{~N}$} & ${ }^{6} \mathrm{~L} \mathbf{i}\left({ }^{1} B, p\right)$ & $E=6-32 \mathrm{MeV}$ & JOUR & Radiochim.Acta 43,139 & $88 \mathrm{Co} 12$ \\
\hline & ${ }^{7} \mathrm{Li}\left({ }^{11} \mathrm{~B}, \mathrm{~d}\right)$ & $E=6-32 \mathrm{MeV}$ & JOUR & Radrochim.Acta 43, 139 & $88 \mathrm{Co} 12$ \\
\hline & ${ }^{8} \mathrm{Li}\left({ }^{18} \mathrm{O}, 2 a\right)$ & $E=10-40 \mathrm{MeV}$ & JOUR & Radiochim.Acta 42,1 & 870 i 07 \\
\hline \multirow[t]{2}{*}{$1+0$} & ${ }^{13} N(p-\gamma)$ & $E=10 \mathrm{w}$ & JOUR & Nucl.Phys. A464, 90 & $87 \mathrm{Fu} 02$ \\
\hline & ${ }^{11} N(p, n)$ & $\mathrm{E}=7-9 \mathrm{MeV}$ & JOUR & Nuclinstr.Meth. Phys.Res. B28. 199 & $87 \mathrm{Ra} 23$ \\
\hline \multirow[t]{4}{*}{${ }^{13} \mathrm{O}$} & $1+N(p, \gamma)$ & $\mathrm{E}=7-9 \mathrm{MeV}$ & JOUR & Nucl.lnstr.Meth. Phys.Res. B28, 199 & $87 R \mathbf{R} 23$ \\
\hline & ${ }^{14} N(p, \gamma)$ & $E=0.2-3.6 \mathrm{MeV}$ & JOUR & Nucl.Phys. A 467, 240 & $875 c 10$ \\
\hline & ${ }^{1+} N(p, \gamma)$ & $E=1165-1185 \mathrm{keV}$ & JOLR & Nucl.Instr.Meth. Phys.Res. A260, 33 & B 7 Se 13 \\
\hline & ${ }^{1+N} N(p, \gamma)$ & $\mathrm{E}=0.5-3.5 \mathrm{MeV}$ & JOUR & Z.Phys. A327, 71 & $\mathrm{B7KrO4}$ \\
\hline \multirow[t]{10}{*}{${ }^{10} \mathrm{O}$} & ${ }^{13} N(p, \gamma)$ & $E=6.25-13.75 \mathrm{MeV}$ & JOUR & Phys.fiev. C37. 2289 & $88 \mathrm{H}$ i 16 \\
\hline & ${ }^{12} \mathrm{C}(\alpha, \gamma)$ & $E=1.29-3.29$ & JOUR & Digs.Abst.Int. 48,1725, Kremer & $87 \mathrm{KJZV}$ \\
\hline & ${ }^{12} c(a, \gamma)$ & $E \approx 0.25-3 \mathrm{MeV}$ & JOUR & Phys.Rev. ( 36,1249 & 87 De 32 \\
\hline & ${ }^{2} \mathrm{c}(a, \gamma)$ & $E=260-360 \mathrm{keV}$ & JOUR & Nucl.Instr.Meth. Phys.Res. A260, 33 & $87 \operatorname{se} 13$ \\
\hline & ${ }^{12} c(\alpha, \gamma)$ & $\mathrm{E}(\mathrm{cm}) \approx 1.5-3 \mathrm{MeV}$ & JoUR & A ust.J.Phys. 40,25 & $87 \mathrm{Ba} 53$ \\
\hline & ${ }^{12} C(\alpha, \gamma)$ & $E=0.94-2.84 \quad \mathrm{MeV}$ & $30 U R$ & Nucl.Phys. A462, 385 & 87 Re02 \\
\hline & ${ }^{12} \mathrm{C}(a, \gamma)$ & $E(\mathrm{~cm})=1.29-3 \mathrm{MeV}$ & JOUR & Phys.Rev.lett. 60, 1475 & $88 \mathrm{~K} r 06$ \\
\hline & ${ }^{13} \mathrm{C}(\alpha, n)$ & $E=10 w$ & JOUR & Phys.Rev. C36, 2206 & 87 De 38 \\
\hline & ${ }^{10} \mathrm{O}\left({ }^{12} \mathrm{C} \cdot{ }^{12} \mathrm{C}\right)$ & $\mathrm{E}(\mathrm{cm}) \leq 8 \mathrm{MeV}$ & JOUR & Phys.Rev. C36. 1408 & $87 \mathrm{~Pa} 24$ \\
\hline & ${ }^{16} \mathrm{O}\left({ }^{16} 0 .{ }^{16} 0\right)$ & $\mathrm{E}(\mathrm{cm}) \leq 12 \mathrm{MeV}$ & JOUR & Phys.Rev. C.36, 1408 & $87 \mathrm{~Pa} 24$ \\
\hline
\end{tabular}


RESIDUAL TARGET-REACTION INCIDENT ENERGY

${ }^{17} \mathrm{O}$

${ }^{15} \mathrm{~N}(\mathrm{~d}, \gamma)$

${ }^{12} \mathrm{C}\left({ }^{3} \mathrm{Li}, \mathrm{d}\right)$

${ }^{17} \mathrm{O}\left({ }^{12} \mathrm{C},{ }^{12} \mathrm{C}\right)$

${ }^{17} \mathrm{O}\left({ }^{13} \mathrm{C}^{13} \mathrm{C}^{1}\right)$

${ }^{18} \mathrm{O}$

$$
\begin{aligned}
& { }^{14} \mathrm{C}(a, \gamma) \\
& { }^{14} \mathrm{C}(\alpha, \gamma) \\
& { }^{13} \mathrm{C}\left({ }^{6} \mathrm{Li}, \mathrm{p}\right) \\
& { }^{18} \mathrm{O}\left({ }^{12} \mathrm{C},{ }^{12} \mathrm{C}^{1}\right)
\end{aligned}
$$

${ }^{19} \mathrm{O} \quad{ }^{6} \mathrm{Li}\left({ }^{18} \mathrm{O}, \mathrm{p} \alpha\right)$

${ }^{16} \mathrm{~F} \quad{ }^{18} \mathrm{O}(\mathrm{p}, \mathrm{n})$

${ }^{17} \mathrm{~F}$

${ }^{16} \mathrm{O}(\mathrm{p}, \gamma)$

${ }^{16} \mathrm{O}(\mathrm{p}, \gamma)$

${ }^{16} \mathrm{O}(\mathrm{p}, \gamma)$

${ }^{18} \mathrm{O}(\mathrm{d}, \mathrm{n})$

${ }^{14} O(\alpha, p)$

${ }^{14} O(\alpha, p)$

${ }^{18} O\left({ }^{16} \mathrm{O},{ }^{15} \mathrm{~N}\right)$

${ }^{18} \mathrm{~F} \quad{ }^{18} \mathrm{O}(\mathrm{p}, \mathrm{n})$

${ }^{27} \mathrm{Al}(\mathrm{p}, 2 \mathrm{n} 2 \alpha)$

${ }^{16} \mathrm{O}(2, n)$

${ }^{16} \mathrm{O}(\mathrm{t}, \mathrm{n})$

${ }^{9} \mathrm{Be}\left({ }^{11} \mathrm{~B}, 2 \mathrm{n}\right)$

${ }^{9} \mathrm{Be}\left({ }^{11} \mathrm{~B}, 2 \mathrm{Zn}\right)$

${ }^{19} \mathrm{~F} \quad{ }^{6} \mathrm{Li}\left({ }^{18} \mathrm{O}, \mathrm{n \alpha}\right)$

${ }^{20} \mathrm{~F} \quad{ }^{10} \mathrm{~B}\left({ }^{14} \mathrm{~B}, \mathrm{p}\right)$

${ }^{1} \mathrm{~B}\left({ }^{\prime \prime} \mathrm{B}, \mathrm{d}\right)$

${ }^{8} \mathrm{Li}\left({ }^{18} \mathrm{O}, \alpha\right)$

${ }^{21} \mathrm{~F} \quad{ }^{6} \mathrm{Li}\left({ }^{18} \mathrm{O},{ }^{3} \mathrm{He}\right)$

${ }^{18} \mathrm{Ne} \quad{ }^{17} \mathrm{~F}(\mathrm{p}, \gamma)$

${ }^{27} \mathrm{Al}(\mathrm{p}, 2 \mathrm{n} 2 \alpha)$

${ }^{14} \mathrm{O}(\alpha, \gamma)$

${ }^{19} \mathrm{Ne} \quad{ }^{19} \mathrm{~F}(\mathrm{p}, \mathrm{n})$

${ }^{15} \mathrm{O}(a, \gamma)$

${ }^{19} \mathrm{~F}(\mathrm{p}, \gamma)$

${ }^{10} \mathrm{~F}(\mathrm{p}, \gamma)$

${ }^{10} \mathrm{~F}(\mathrm{p}, \gamma)$

${ }^{10} \mathrm{O}(a, \gamma)$

${ }^{10} \mathrm{O}(\alpha, \gamma)$

${ }^{10} \mathrm{O}(\alpha, \gamma)$

${ }^{B} \mathrm{O}\left({ }^{\mathrm{B}} \mathrm{Li}, \mathrm{P}, \mathrm{p}\right)$

\begin{tabular}{|c|c|}
\hline${ }^{22} \mathrm{Ne}$ & $\begin{array}{l}{ }^{18} \mathrm{O}(\alpha, \gamma) \\
{ }^{0} \mathrm{Li}\left({ }^{18} \mathrm{O}, \mathrm{d}\right)\end{array}$ \\
\hline${ }^{23}$ Ne & $\begin{array}{l}{ }^{8} \mathrm{Li}\left({ }^{18} \mathrm{O}, \mathrm{p}\right) \\
{ }^{6} \mathrm{Li}\left({ }^{18} \mathrm{O}, \mathrm{p}\right)\end{array}$ \\
\hline $2 ! \mathrm{Na}$ & ${ }^{20} \mathrm{Ne}(p, \gamma)$ \\
\hline${ }^{22} \mathrm{Na}$ & $\begin{array}{l}{ }^{24} M g(d, \alpha) \\
\operatorname{Li}(\alpha, n) \\
\theta_{B e}(\alpha, n)\end{array}$ \\
\hline
\end{tabular}

${ }^{18} \mathrm{O}\left({ }^{6} \mathrm{Li}, \mathrm{d}\right)$

${ }^{12} \mathrm{C}\left({ }^{12} \mathrm{C}, \alpha\right)$

${ }^{18} \mathrm{O}\left({ }^{10} \mathrm{O},{ }^{12} \mathrm{C}\right)$
$\mathrm{E}=30 \mathrm{MeV}$

$E(\mathrm{~cm})=7.4,9.4 \mathrm{MeV}$

$E(\mathrm{~cm}) \approx 22-32 \mathrm{MeV}$

$E(\mathrm{~cm}) \approx 10-20 \mathrm{MeV}$

$E=1.7-2.7 \mathrm{MeV}$

E not given

$\mathrm{E}=28 \mathrm{MeV}$

$E \approx 10-20 \mathrm{MeV}$

$E=10-40 \mathrm{MeV}$

$E=7-9 \mathrm{NeV}$

$E=40,65,85 \mathrm{Me} \mathrm{V}$

$E=7-9 \mathrm{MeV}$

$E=16 \mathrm{MeV}$

$E<14.7 \mathrm{MeV}$

$E \approx 0.5-4 \mathrm{MeV}$

$E$ nat given

$E=72 \mathrm{MeV}$

$\mathrm{E}<14.7 \mathrm{MeV}$

$E=13.3-67.5 \mathrm{MeV}$

$\mathrm{E}<14.7 \mathrm{MeV}$

$\mathrm{E}=3-12 \mathrm{Me} \mathrm{V}$

$\mathrm{E}=6-32 \mathrm{MeV}$

$\mathrm{E}=6-32 \mathrm{MeV}$

$E=10-40 \mathrm{MeV}$

$E=6-32 \mathrm{MeV}$

$E=6-32 \mathrm{MeV}$

$E=10-40 \mathrm{MeV}$

$E=10-40 \mathrm{MeV}$

$\mathrm{E}(\mathrm{cm}) \leq 0.7 \mathrm{MeV}$

$E=13.3-67.5 \mathrm{MeV}$

E not given

$E=7-9 \mathrm{MeV}$

$\mathrm{E}=0-2 \mathrm{MeV}$

$E=3-10 \mathrm{MeV}$

$E=7-9 \mathrm{MeV}$

$E=3.5-13.3 \mathrm{MeV}$

$E(\mathrm{~cm})=1.7-2.35 \mathrm{MeV}$

$E=15-40 \mathrm{MeV}$

E not given

$E(\mathrm{~cm})=7.2-13.3 \mathrm{MeV}$

$\mathrm{E}(\mathrm{cm})=7.4,9.4 \mathrm{MeV}$

$\mathrm{E}(\mathrm{cm})=4.7-6 \mathrm{MeV}$

$E=72 \mathrm{MeV}$

$E=0-2 \mathrm{MeV}$

$E=10-40 \mathrm{MeV}$

$E=10-40 \mathrm{MeV}$

$E=10-40 \mathrm{MeV}$

$E=300-400 \mathrm{keV}$

$E=14 \mathrm{MeV}$

$E \leq 10 \mathrm{MeV}$

$E \leq 10 \mathrm{MeV}$

\section{REFERENCE}

KEY NUMBER

JOUR Nucl.Phys. A483, 9

JOUR Phys.Rev. C35, 1746

JOUR Phys.Rev. C36, 2341

JOUR Phys.Rev. C36, 2341

JouR Phys.Rev. C36, 1256

JOUR Astrophys.J. 324, 953

JoUR Phys.Rev. C37, 1036

JOUR Phys.Rev. C36, 2341

JoUR Radiochim. Acta 42, 1

JouR Nucl.lnstr.Meth. Phys.Res. B28, 199

$88 \operatorname{Co} 10$

87 Ar 13

$87 \mathrm{Mo27}$

$87 \mathrm{Mo27}$

87Ga 15

88Bu01

88Sm01

B7Ho27

87Di07

B7Ra23

JOUR Phys.Rev. C35, 1214

JouR Nucl.Instr. Meth. Phys.Res. B28, 199

JouR Nucl.Phys. A483, 9

JouR J.Radioanal.Nucl.Chem. 102, 159

JOUR Nucl.Phys. A480, 188

JOUR Astrophys.J. 316, 162

JOUR Nucl.Phys. A 481,577

JOUR J.Radioanal.Nucl.Chem. 102, 159

JouR Appl.Radiat.isot. 39, 41

JoUR J.P.adioanal.Nucl.Chem. 102, 159

JoUR Nucl.Instr. Meth. Phys.Res. B24/25, 722

JoUR Radiochim. Acta 43, 139

JOUR Radiochim.Acta 43, 139

JOUR Radiochim.Acta 42, 1

B7La 11

87Ra23

BBCO10

B6Ai 04

B8Fu02

B7W i 11

88Au03

$86 \mathrm{Ai04}$

BBLa 01

B6Ai 04

B7Bo 16

$8 B C 012$

BBCo12

B7Dj07

JouR Radiochim.Acta 43, 139

JOUR Radiochim.Acla 43, 139

JOUR Radiochim. Acta 42, 1

$8 B \operatorname{Co} 12$

BBCo12

B7Di07

JOUR Radiochim.Acta 42, 1

87Dj07

JOUR Astrophys.J. 326, 384

JouR Appl.Radiat.lsat. 39, 41

JOUR Astrophys.J. 316, 162

B8w i 08

8BLa 01

87Wi 11

JouR Nucl.Instr.Meth. Phys.Res. B28, 199

B7Ra23

B8Bu0 1

JouR Phys.Rev. C37, 2281

JOUR Nucl.Instr.Meth. Phys.Res. B28, 199

$88 \mathrm{Ku08}$

B7Ra23

BBWa13

$87 \mathrm{Ha} 24$

B7MiOT

BBBu01

B7Ar 13

B7Ar13

B8De 18

$88 \mathrm{Au} 03$

JoUR Nucl.Phys. A481, 577

888401

B7D 07

JOUR Radiochim. Acta 42, 1

B7DI07

87Di07

JOUR Radiochimicta 42,

87Se 13

JOUR J.Radjoanal.NucJ.Chem. 127, 31

JoUR At.Energ. 62, 232

JOUR At.Energ. 62, 232
BBDe20

87Vu02

B7Vu02 
RESIDUAL TARGET-REACTION INCIDENT ENERGY

${ }^{22} \mathrm{Na} \quad \mathrm{B}(\alpha, \mathrm{n})$

$C(\alpha, n)$

${ }^{19} p(a, n)$

${ }^{12} \mathrm{C}\left({ }^{12} \mathrm{C}, \mathrm{np}\right)$

${ }^{12} \mathrm{C}\left({ }^{12} \mathrm{C}, \mathrm{np}\right)$

${ }^{12} \mathrm{C}\left({ }^{12} \mathrm{C}, \mathrm{d}\right)$

\begin{tabular}{|c|c|c|}
\hline${ }^{23} \mathrm{Na}$ & $\begin{array}{l}{ }^{26} \mathrm{Mg}(p, \alpha) \\
{ }^{27} \mathrm{Al}(\mathrm{p}, 2 \mathrm{n} 3 \mathrm{p}) \\
{ }^{12} \mathrm{C}\left({ }^{12} \mathrm{C}, \mathrm{p}\right) \\
{ }^{6} \mathrm{Li}\left({ }^{18} \mathrm{O}, \mathrm{n}\right)\end{array}$ & $\begin{array}{l}E=5.3-7 \mathrm{MeV} \\
E=13.3-67.5 \mathrm{MeV} \\
E(\mathrm{~cm})=4.7-6 \mathrm{MeV} \\
E=10-40 \mathrm{MeV}\end{array}$ \\
\hline${ }^{24} \mathrm{Na}$ & ${ }^{27} \mathrm{Al}(p, n 3 p)$ & $E=13.3-67.5 \mathrm{MeV}$ \\
\hline${ }^{23} \mathrm{Mg}$ & $\begin{array}{l}{ }^{23} \mathrm{Na}(\mathrm{p}, \mathrm{n}) \\
{ }^{12} \mathrm{C}\left({ }^{12} \mathrm{C}, \mathrm{n}\right)\end{array}$ & $\begin{array}{l}E=7-9 \mathrm{MeV} \\
E(\mathrm{~cm})=4.7-6 \mathrm{MeV}\end{array}$ \\
\hline${ }^{24} \mathrm{Mg}$ & $\begin{array}{l}{ }^{23} \mathrm{Na}(\mathrm{p}, \gamma) \\
{ }^{23} \mathrm{Na}(\mathrm{p}, \gamma) \\
{ }^{12} \mathrm{C}\left({ }^{12} \mathrm{C}, \gamma\right) \\
{ }^{16} \mathrm{O}\left({ }^{18} \mathrm{O}, \mathrm{X}\right) \\
{ }^{28} \mathrm{Si}\left({ }^{32} \mathrm{~S},{ }^{36} \mathrm{Ar}\right)\end{array}$ & $\begin{array}{l}E=7-9 \mathrm{MeV} \\
E=220-340 \mathrm{keV} \\
E(\mathrm{~cm})=4.7-6 \mathrm{MeV} \\
E(\mathrm{~cm})=8-13.8 \mathrm{MeV} \\
E=42-48.1 \mathrm{MeV}\end{array}$ \\
\hline${ }^{26} \mathrm{Mg}$ & ${ }^{26} \mathrm{Mg}\left(\mathrm{p}, \mathrm{p}^{\prime}\right)$ & $E=5.3-6.1 \mathrm{MeV}$ \\
\hline${ }^{27} \mathrm{Mg}$ & $\begin{array}{l}{ }^{25} \mathrm{Mg}(\mathrm{t}, \mathrm{p}) \\
{ }^{27} \mathrm{~A} I\left({ }^{7} \mathrm{Li},{ }^{7} \mathrm{Be}\right)\end{array}$ & $\begin{array}{l}E=3-12 \mathrm{MeV} \\
E=78 \mathrm{MeV}\end{array}$ \\
\hline${ }^{28} \mathrm{Mg}$ & ${ }^{20} \mathrm{Mg}(\mathrm{t}, \mathrm{p})$ & $\mathrm{E}=3-12 \mathrm{MeV}$ \\
\hline${ }^{24} \mathrm{Al}$ & ${ }^{24} \mathrm{Mg}(p, n)$ & $E=7-9 \mathrm{MeV}$ \\
\hline${ }^{25} \mathrm{Al}$ & ${ }^{24} \mathrm{Mg}(p, \gamma)$ & $E=7-9 \mathrm{MeV}$ \\
\hline${ }^{26} \mathrm{Al}_{1}$ & $\begin{array}{l}{ }^{23} \mathrm{Mg}(\mathrm{p}, \gamma) \\
{ }^{26} \mathrm{Mg}(\mathrm{p}, \mathrm{n}) \\
{ }^{28} \mathrm{Mg}(\mathrm{p}, \mathrm{n}) \\
{ }^{23} \mathrm{Na}(\alpha, \mathrm{n}) \\
{ }^{12} \mathrm{C}\left({ }^{16} \mathrm{O}, \mathrm{np}\right) \\
{ }^{12} \mathrm{C}\left({ }^{16} \mathrm{O}, \mathrm{d}\right)\end{array}$ & $\begin{array}{l}E \approx \text { resonance } \\
E=3.9-5.9 \mathrm{MeV} \\
E=7-9 \mathrm{MeV} \\
E=5.3-6.1 \mathrm{MeV} \\
E(\mathrm{~cm})=11-16 \mathrm{MeV} \\
E(\mathrm{~cm})=13.8-22.1 \mathrm{MeV}\end{array}$ \\
\hline${ }^{27} \mathrm{Al}$ & $\begin{array}{l}{ }^{26} \mathrm{Mg}(\mathrm{p}, \gamma) \\
{ }^{13} \mathrm{C}\left({ }^{16} \mathrm{O}, \mathrm{np}\right) \\
{ }^{13} \mathrm{C}\left({ }^{16} \mathrm{O}, \mathrm{d}\right) \\
{ }^{16} \mathrm{O}\left({ }^{18} \mathrm{O}, \mathrm{X}\right)\end{array}$ & $\begin{array}{l}E=7-9 \mathrm{MeV} \\
E(\mathrm{~cm})=11-16 \mathrm{MeV} \\
E(\mathrm{~cm})=13.8-22.1 \mathrm{MeV} \\
E(\mathrm{~cm})=8-13.8 \mathrm{MeV}\end{array}$ \\
\hline${ }^{2 \theta} \mathrm{Al}$ & ${ }^{28} \mathrm{Si}\left({ }^{7} \mathrm{Li},{ }^{7} \mathrm{Be}\right)$ & $E=78 \mathrm{MeV}$ \\
\hline${ }^{29} \mathrm{Al}$ & ${ }^{27} \mathrm{~A} I(t, p)$ & $E=3-12 \mathrm{MeV}$ \\
\hline${ }^{27} \mathrm{Si}$ & $\begin{array}{l}{ }^{26} \mathrm{~A} I(p, \gamma) \\
{ }^{27} \mathrm{~A}(p, \mathrm{n})\end{array}$ & $\begin{array}{l}E=0.05-0.15 \mathrm{MeV} \\
E=7-9 \mathrm{MeV}\end{array}$ \\
\hline${ }^{28} \mathrm{Si}$ & $\begin{array}{l}{ }^{27} \mathrm{Al}(\mathrm{p}, \gamma) \\
{ }^{27} \mathrm{~A} 1(\mathrm{p}, \gamma) \\
{ }^{28} \mathrm{Si}(\mathrm{p}, \mathrm{p}) \\
{ }^{16} \mathrm{O}\left({ }^{16} \mathrm{O}, \mathrm{a}\right) \\
{ }^{16} \mathrm{O}\left({ }^{18} \mathrm{O}, \mathrm{X}\right) \\
{ }^{26} \mathrm{Si}\left({ }^{32} \mathrm{~S},{ }^{32} \mathrm{~S}\right)\end{array}$ & $\begin{array}{l}E=7-9 \mathrm{MeV} \\
E=200-360 \mathrm{keV} \\
E \leq 40 \mathrm{MeV} \\
E=12-20 \mathrm{MeV} \\
E(\mathrm{~cm})=8-13.8 \mathrm{MeV} \\
E=42-48.1 \mathrm{MeV}\end{array}$ \\
\hline${ }^{2 \theta} \mathrm{Si}$ & ${ }^{27} \mathrm{Al}\left({ }^{3} \mathrm{He}, \mathrm{p}\right)$ & $\mathrm{E}=2-20 \mathrm{MeV}$ \\
\hline${ }^{30} \mathrm{Si}$ & ${ }^{16} \mathrm{O}\left({ }^{16} \mathrm{O}, \mathrm{X}\right)$ & $E(\mathrm{~cm})=8-13.8 \mathrm{MeV}$ \\
\hline${ }^{28} \mathbf{g}_{P}$ & ${ }^{28} \operatorname{Si}(p, n)$ & $\mathrm{E}=7-9 \mathrm{MeV}$ \\
\hline
\end{tabular}

$\mathrm{E} \leq 10 \mathrm{MeV}$
REFERENCE

Es $10 \mathrm{MeV}$

Es $10 \mathrm{MeV}$

$E(\mathrm{~cm})=11-16 \mathrm{MeV}$

$\mathrm{E}(\mathrm{cm})=13.8-22.1 \mathrm{MeV}$

$E(\mathrm{~cm})=7.2-13.3 \mathrm{MeV}$

$$
\begin{aligned}
& E=5.3-7 \mathrm{MeV} \\
& E=13.3-67.5 \mathrm{MeV} \\
& E(\mathrm{~cm})=4.7-6 \mathrm{Me} \\
& E=10-40 \mathrm{MeV} \\
& E=13.3-67.5 \mathrm{MeV} \\
& E=7-9 \mathrm{MeV}
\end{aligned}
$$

$\mathrm{E}=7-9 \mathrm{MeV}$

$E(c m)=8-13.8 \mathrm{MeV}$

$E=5.3-6.1 \mathrm{MeV}$

$E=3-12$ MeV

$\mathrm{E}=3-\mathrm{I} 2 \mathrm{MeV}$

$\mathbf{E}=7-9 \mathrm{MeV}$

$E \approx$ resonance

$E=3.9-5.9$ MeV

$E(\mathrm{~cm})=11-16 \mathrm{MeV}$

$E=7-9 \mathrm{MeV}$

$E(\mathrm{~cm})=13.8-22.1 \mathrm{MeV}$

$E=78 \mathrm{MeV}$
JOUR At.Energ. 62, 232

JOUR At.Energ. 62, 232

JOUR At.Energ. 62, 232

JOUR Phys.Rev, C35, 1746

JouR Phys.Rev. C35, 1746

JouR Phys.Rev. C35, 1746

JOUR Nucl.Phys. A467, 511

JOUR Appl.Radiat.Isot. 39, 41

JOUR Z.Phys. A330, 93

JOUR Radiochim.Acta 42, I

JOUR Appl.Radiat.lsot. 39,41

KEY NUMBER

JouR Nucl.Instr.Meth. Phys.Res. B28, 199

JOUR Z.Phys. A 330, 93

JOUR Nucl.Instr.Meth. Phys.Res. B28, 199

JoUR Nucl.Instr.Meth. Phys.Res. A260, 33

JOUR Z.Phys. A330, 93

JOUR Phys.Rev. C35, 591

JOUR Nucl.Phys. A463, 731

JoUR Nucl.Phys, A467, 511

$87 \mathrm{VuO2}$

BᄀVu02

B7vu02

87 Ar 13

B7Ar 13

G7Ar 13

87Do06

$88 \mathrm{LaO} 1$

88De 18

$87 \mathrm{Di} 07$

B8La01

87 Ra23

88De 18

87Ra23

87Se 13

$88 \mathrm{De} 18$

$87 \mathrm{Ku02}$

$87 \mathrm{~B}, 03$

87 Do06

JoUR Nucl.Instr.Meth. Phys.Res, B24/25, 722 JOUR Yad.Fiz. 45, 1236

$87 \mathrm{Bo} 16$

870105

JOUR Nucl.lnstr.Meth. Phys.Res. B24/25, 722

$87 \mathrm{Bo} 16$

JouR Nucl.lnstr.Meth. Phys.Res. B28, 199

$87 \mathrm{Ha} 23$

JOUR Nucl.Instr.Meth. Phys.Res. B28, 199

87Ra23

JOUR Nucl.Phys. A467, 261

87Eก02

87Do06

87 R23

87 Do06

$87 \mathrm{Ar} 13$

$87 \operatorname{Ar} 13$

JOUR Phys.Rev. C35, 1746

JOUR Phys.Rev, C35, 1746

JOUR Nuct.Instr.Meth. Phys.Res. B28, 199

$87 \mathrm{Ra} 23$

$87 \operatorname{Ar} 13$

87 Ar 13

$87 \mathrm{KuD2}$

JOUR Phys.Rev C35, 1746

JOUR Phys.Rev. C35, 591

876105

JOUR Yad.Fiz. 45,1236

$87 B 016$

JOUR Nucl.lnstr.Meth. Phys.Res. B24/25, 722

$87 \mathrm{Ra} 26$

JOLR Astrophys.J. 320, 405

87Ra23

JOUR Nucl.Instr.Meth. Phys.Res. B28, 199

JOUR Nucl.Phys. A477, 105

JOUR Izy.Akad.Nauk SSSR, Ser.Fiz. 51, 24

JOUR Phys.ReviLett. 58, 1512

JOUR Phys.Rev. C35, 591

JOUR Nucl.Phys. A463, 731

$87 \operatorname{Ra} 23$

B日T:01

$87 \mathrm{Ro07}$

B7Ga03

$87 \mathrm{KuO2}$

$87 \mathrm{~B}, 03$

JOUR Rep.Prog.Phys. 50, 1171

$87 \mathrm{HO} 2$

JOUR Phys.Rev. C35, 591

$87 K u 02$

JOUR Nucl.instr.Meth. Phys.Res. B28, 199

$87 \mathrm{Ha} 23$ 
RESIDUAL TARGET-REACTION INCIDENT ENERGY

\begin{tabular}{|c|c|}
\hline${ }^{29} \mathrm{P}$ & $\begin{array}{l}{ }^{2 \theta} \mathrm{Si}(p, \gamma) \\
{ }^{29} \mathrm{Si}(p, n)\end{array}$ \\
\hline${ }^{30} \mathrm{P}$ & $\begin{array}{l}{ }^{29} \mathrm{Si}(p, \gamma) \\
{ }^{30} \mathrm{Si}(p, n) \\
{ }^{28} \mathrm{Si}(t, n) \\
{ }^{24} \mathrm{Mg}\left({ }^{11} \mathrm{~B}, \mathrm{n \alpha}\right) \\
{ }^{25} \mathrm{Mg}\left({ }^{11} \mathrm{~B}, 2 \mathrm{n} \alpha\right. \\
{ }^{26} \mathrm{Mg}\left({ }^{11} \mathrm{~B}, 3 n \alpha\right) \\
{ }^{16} \mathrm{O}\left({ }^{16} \mathrm{O}, \mathrm{np}\right) \\
{ }^{16} \mathrm{O}\left({ }^{16} \mathrm{O}, \mathrm{d}\right) \\
{ }^{16} \mathrm{O}\left({ }^{16} \mathrm{O}, \mathrm{X}\right)\end{array}$ \\
\hline${ }^{31} \mathrm{P}$ & $\begin{array}{l}{ }^{30} \mathrm{Si}(\mathrm{p}, \gamma) \\
{ }^{16} \mathrm{O}\left({ }^{18} \mathrm{O}, \mathrm{X}\right)\end{array}$ \\
\hline${ }^{31} \mathrm{~S}$ & $\begin{array}{l}{ }^{1} P(p, n) \\
{ }^{16} O\left({ }^{16} 0, x\right)\end{array}$ \\
\hline${ }^{32} \mathrm{~S}$ & $\begin{array}{l}{ }^{31} P(p, \gamma) \\
{ }^{32} S(p, p)\end{array}$ \\
\hline${ }^{34} \mathrm{~S}$ & $\begin{array}{l}{ }^{30} \mathrm{~S}_{1}\left({ }^{6} \mathrm{~L}, \mathrm{n},\right. \\
{ }^{30} \mathrm{Si}_{1}\left({ }^{6} \mathrm{Ll}, \mathrm{d}\right)\end{array}$ \\
\hline${ }^{32}(1)$ & ${ }^{32} S(p, n)$ \\
\hline${ }^{33} \mathrm{Cl}$ & ${ }^{32} S(p, \gamma)$ \\
\hline${ }^{34} \mathrm{Cl}$ & $\begin{array}{l}{ }^{32} \mathrm{~S}(\mathrm{t}, \mathrm{n}) \\
{ }^{35} \mathrm{Cl}(\alpha, \mathrm{n} \alpha) \\
{ }^{20} \mathrm{Si}\left({ }^{11} \mathrm{~B}, \mathrm{n} \alpha\right) \\
{ }^{29} \mathrm{Si}\left({ }^{11} \mathrm{~B}, 2 \mathrm{n} \alpha\right) \\
{ }^{30} \mathrm{Si}\left({ }^{11} \mathrm{~B}, 3 \mathrm{n} \alpha\right)\end{array}$ \\
\hline${ }^{35} \mathrm{Ar}$ & ${ }^{35} \mathrm{Cl}(p, n)$ \\
\hline${ }^{36}$ A S & $\begin{array}{l}{ }^{35} \mathrm{CJ}(p . \gamma) \\
{ }^{36} \mathrm{Ar}\left(\mathrm{p} . p^{\prime}\right)\end{array}$ \\
\hline${ }^{37} \mathrm{~K}$ & ${ }^{38} \mathrm{Ar}(\mathrm{p}, \gamma)$ \\
\hline${ }^{38} \mathrm{~K}$ & $\begin{array}{l}{ }^{35} \mathrm{Cl}(\alpha, n) \\
{ }^{35} \mathrm{Cl}(\alpha, n) \\
{ }^{20} \mathrm{Si}\left({ }^{12} \mathrm{C}, n \mathrm{p}\right) \\
{ }^{26} \mathrm{Si}\left({ }^{12} \mathrm{C}, \mathrm{d}\right)\end{array}$ \\
\hline${ }^{38} \mathrm{Ca}$ & ${ }^{39} K(p, n)$ \\
\hline${ }^{40} \mathrm{Ca}$ & $\begin{array}{l}{ }^{38} \mathrm{~K}(p, \gamma) \\
{ }^{40} \mathrm{Ca}\left(p, p^{\prime}\right)\end{array}$ \\
\hline${ }^{41} \mathrm{Ca}$ & $\begin{array}{l}\mathrm{Ti}(p, X) \\
\operatorname{Pe}(p, X) \\
\mathrm{Ni}(p, X)\end{array}$ \\
\hline$+2 \mathrm{Ca}$ & ${ }^{39} \mathrm{~K}(\alpha, p)$ \\
\hline${ }^{40} \mathrm{Sc}$ & ${ }^{40} \mathrm{Ca}(\mathrm{P}, \mathrm{n})$ \\
\hline${ }^{41 S C}$ & $\begin{array}{l}{ }^{40} \mathrm{Ca}(p, \gamma) \\
{ }^{40} \mathrm{Ca}(p, \gamma)\end{array}$ \\
\hline $46 \mathrm{~V}$ & ${ }^{46} \mathrm{~T} 1(p, n)$ \\
\hline $47 \mathrm{~V}$ & ${ }^{4 \theta} T_{1}(p, \gamma)$ \\
\hline
\end{tabular}

$\mathrm{E}=7-9 \mathrm{MeV}$

$E=7-9$ MeV

$\mathrm{E}=7-9 \mathrm{MeV}$

$\mathrm{E}=7-9 \mathrm{MeV}$

$\mathrm{E}=3-12 \mathrm{MeV}$

$E=6-32 \mathrm{MeV}$

$E=6-32 \mathrm{MeV}$

$E=6-32 \mathrm{MeV}$

$\mathrm{E}(\mathrm{cm})=11-16 \mathrm{MeV}$

$E(\mathrm{~cm})=13.8-22.1 \mathrm{MeV}$

$E(\mathrm{~cm})=8-13.8 \mathrm{MeV}$

$\mathrm{E}=7-9 \mathrm{MeV}$

$E(\mathrm{~cm})=8-13.8 \mathrm{MeV}$

$E=7-9 \mathrm{MeV}$

$E(\mathrm{~cm})=8-13.8 \mathrm{MeV}$

$\mathrm{E}=7-9 \mathrm{MeV}$

$\mathrm{E} \leq 40 \mathrm{MeV}$

$E(\mathrm{~cm})=7.2 \quad 13.3 \mathrm{MeV}$

$E(c / n)=7.4 .9 .4 \mathrm{MeV}$

$\mathrm{E}=7-9 \mathrm{MeV}$

$\mathrm{E}=7-\mathrm{g} \mathrm{MeV}$

$E=3-12 \mathrm{MeV}$

$E=$ threshold $-26 \mathrm{MeV}$

$E=6-32 \mathrm{MeV}$

$\mathrm{E}=6-32 \mathrm{MeV}$

$\mathrm{E}=6-32 \mathrm{MeV}$

$E=7-9 \mathrm{MeV}$

$E=7-9 \quad M e V$

$E=0.9-3.3 \mathrm{MgV}$

$\mathrm{E}-2.6-3.3 \mathrm{MeV}$

$E=15-30 \mathrm{MeV}$

$E=t$ hreshold-26 MeV

$E(\mathrm{~cm})=13.8-22.1 \mathrm{MeV}$

$E(\mathrm{~cm})=7.2-13.3 \mathrm{MeV}$

$E=7-9 \mathrm{MeV}$

$E=7-9 \quad M e V$

$E \approx 10-40 \mathrm{MeV}$

$E=40-600 \mathrm{MeV}$

$E=40-600 \mathrm{MeV}$

$E=40-600 \mathrm{MeV}$

$\mathrm{E}=4.5-9 \mathrm{MeV}$

$E=7-9$ MeV

$E=640-3500 \mathrm{keV}$

$E=7-9 \mathrm{MeV}$

$E=7-9$ MeV

$\mathrm{E}=7-9 \mathrm{MeV}$

\section{REFERENCE}

JOUR Nucl.1nstr.Meth. Phys.Res. B28, 199

JOUR Nuclinstr.Meth. Phys.Res. B28, 199

KEY NUMBER

JouR Nuclinstr.Meth. Phys.hes. B28, 199

JoUR Nucl.Instr.Meth. Phys.Res. B28, 199

JoliR Nucl.Instr.Meth. Phys.Hes, B24:25, 722

JOL'R Radjochim.Acta 43, 139

JOLR Radjochim. Acta 43, 139

JOLR Radiochim.Acta 43, 139

JouR Phys.Rev. C35, 1746

JOUR Phys Rev. C35, 1746

JOUR Phys.Rev. C35, 591

Jouk Nucl.Jnstr.Meth. Phys.Res. B28, 199

JoUR Phys.Rev, C.35, 591

Jour Nucl.lnstr.Meth. Phys.Res, B28, 199

Jour Phys.rev. (*35, 591

JotiR Nuclinstr.Meth. Phys.Res. B28. 199

JoliR lzv.Akad.Nauk SSSR, Ser.Fiz. 51, 24

JoUR Phys.Rev. C35, 1746

JOLR PhysRev. 035,1746

JOUR Nucl.lnstr.Meth. Phys.Res. B28. 199

B7Ra:3

B7Ra23

$87 R a 23$

87 หa23

878016

$88 \mathrm{Cot} 2$

$88 \mathrm{Co} 12$

$88 \mathrm{Col} 2$

87 Ar 13

87 N 13

$87 \mathrm{Ku} 02$

$87 \mathrm{Ra} 2.3$

87KuO2

87 Ra 23

$87 \mathrm{KuO}$

$87 \mathrm{Ra} 23$

87Ro07

87A 13

87AT 13

g7Ra 23

Jolk Nucl.Instr.Meth. Phys.Res. B28, 199

B7Ra23

JOLR Nucl.Instr.Meth. Phys.Res, B24/25, 722

Jol:R Appl.Radiat.lsot. 39. 479

JoUA Radiochim.Acta 43, 139

JOUR Radiochim.Acta 43, 139

JOUR Radiochim.Acta 43. 139

JouR Nucl.lnstr.Meth. Phys.Res. B28. 199

JoUk Nucilnstr.Meth. Phys.hes. B2B. 199

JoUR Nucl.Phys. A476, 316

JoUR Nucl.Phys, $\wedge 476,316$

Jolth Appl. Radial.Isot. 39, 97

JoL A Apl.Radiat.Isot. 39, 479

JOUR Phys,Rev. C35, 1746

JOUR Phys.Rev. C35, 1746

JoUR Nuclinstr.Meth. Phys.Res. B28, 199

JoUR Nucl.lnstr.Meth. Phys.Res. B28, 199

JOUR Phys.Rev. C37.906

JoUR Nuclinstr.Meth. Phys.Res. B29, 275 JOUR Nucl.instr.Meth. Phys.Res. B29. 275 JOLR Nucl.Instr.Meth. Phys.Res. B29, 275

JOLR Nucl.Phys. A475, 548

$87 \mathrm{Bo16}$

$880 \mathrm{0} 01$

$88 \mathrm{CO} 12$

88 Co 12

B8Colz

$87 \mathrm{Ka23}$

B7Ha2:

8BDeOJ

880003

88Gu01

88Qa01

$87 \mathrm{Ar} 13$

g7ar 13

87Ra23

87Ra23

88B007

$87 f+1]$

87F」11

B7FI1 1

B7ste 27

JoLh Nuclinstr. Meth. Phys.Res. B28, 199

87Razis

JoUR Nuel Phys A 466,280

$87 \% 102$

JOLR Nuclinstr.Meth. Phys.Res, B28, is9

87 Ra23

JoliR Nucl instr.Meth. Phys.Res. B28. 199

B7Raz3

JoUR Nucl.Instr. Meth. Phys.Res. H:8, 199 
RESIDUAL TARGET-REACTION INCIDENT ENERGY

${ }^{47} \mathrm{~V} \quad{ }^{47} \mathrm{Ti}(\mathrm{p}, \mathrm{n})$

$48 \mathrm{~V}$

${ }^{47} \mathrm{Ti}(\mathrm{p}, \gamma)$

$4 \mathrm{~T} i(p, n)$

$49 \mathrm{~V}$

${ }^{48} \mathrm{~T}:(\mathrm{p}, \gamma)$

${ }^{51} \mathrm{Cr} \quad \mathrm{Ni}(\mathrm{p}, \mathrm{X})$

${ }^{39} \mathrm{Co}(\alpha, 3 \mathrm{np} 2 \alpha)$

${ }^{32} \mathrm{Mn} \quad{ }^{32} \mathrm{Cr}(\mathrm{p}, \mathrm{n})$

${ }^{5} \mathrm{Cr}(\mathrm{p}, \mathrm{n})$

$\operatorname{Cr}(p, x)$

$\mathrm{Ni}(\mathrm{p}, \mathrm{X})$

${ }^{52} \operatorname{Cr}(d, 2 n)$

${ }^{52} \mathrm{Cr}(\mathrm{d}, 2 \mathrm{n})$

${ }^{1} \mathrm{~V}(\alpha, 3 n)$

${ }^{98} \mathrm{Co}(\alpha, 3 \mathrm{n} 2 \alpha)$

${ }^{31} \mathrm{Mn} \quad{ }^{52} \mathrm{Cr}(\mathrm{p}, \gamma)$

${ }^{54} \mathrm{Mn} \quad{ }^{51} \mathrm{~V}(\alpha, n)$

${ }^{59} \mathrm{Co}(\alpha, n 2 \alpha)$

${ }^{59} \mathrm{Co}(\alpha, 5 n 4 \mathrm{p})$

${ }^{55} \mathrm{Mn} \quad{ }^{55} \mathrm{Mn}\left(\mathrm{p}, \mathrm{p}^{\prime}\right)$

${ }^{55} \mathrm{Mn}\left(\mathrm{p}, \mathrm{p}^{\prime}\right)$

${ }^{55} \mathrm{Fe} \quad{ }^{53} \mathrm{Mn}(\mathrm{p}, \mathrm{n})$

${ }^{s 5} \mathrm{Mn}(p, n)$

${ }^{5 s} M n(p, n)$

${ }^{56} \mathrm{Fe} \quad{ }^{55} \mathrm{Mn}(\mathrm{p}, \gamma)$

${ }^{55} \mathrm{Mn}(\mathrm{p}, \gamma)$

${ }^{55} \mathrm{Mn}(p, \gamma)$

${ }^{s 9} \mathrm{Fe} \quad \mathrm{Cu}(p, X)$

${ }^{9} \mathrm{Co}(\alpha, n 3 p)$

${ }^{54} \mathrm{Co} \quad{ }^{54} \mathrm{Fe}(\mathrm{p}, \mathrm{n})$

${ }^{55} \mathrm{Co} \quad{ }^{44} \mathrm{Fe}(\mathrm{p}, \gamma)$

st Fe $(p, \gamma)$

${ }^{3 \theta} \mathrm{Fe}(\mathrm{p}, \mathrm{n})$

Ni $(p, x)$

$\mathrm{Cu}(p, x)$

${ }^{59} \mathrm{Co}(\alpha, 3 \mathrm{n} \alpha)$

${ }^{50} \mathrm{Co}(\alpha, 5 n 2 p)$

$F e(\alpha, x \cap p)$

${ }^{52} \mathrm{Co}$

${ }^{36} \mathrm{Fe}(p, \gamma)$

${ }^{5 \theta} \mathrm{Co}(\mathrm{p}, 2 \mathrm{np})$

$\mathrm{Ni}(p, x)$

Cu $(p, X)$

${ }^{50} \mathrm{Co}(\alpha, 2 \mathrm{n} \alpha)$

${ }^{3 \theta} \mathrm{Co}(\alpha, 4 \pi 2 p)$

$\operatorname{Fe}(\alpha, x \cap p)$

$5 \mathrm{Co}$

${ }^{99} \mathrm{Co}(p, n p)$

Ni $(p, X)$

$\mathrm{Cu}(\mathrm{p}, \mathrm{x})$

${ }^{58} \mathrm{Co}(\alpha, n \alpha)$

${ }^{9} \mathrm{Co}(\alpha, 3 \mathrm{n} 2 \mathrm{p})$

$\operatorname{Fe}(\alpha, x \cap \mathrm{p})$

${ }^{50} \mathrm{Ne}\left({ }^{7} \mathrm{Li},{ }^{7} \mathrm{Be}\right)$
$E=7-9 \mathrm{MeV}$

$E=7-9 \mathrm{MeV}$

$\mathrm{E}=7-9 \mathrm{MeV}$

$\mathrm{E}=7-9 \mathrm{MeV}$

$E=10-100 \mathrm{MeV}$

$\mathrm{E}=10-120 \mathrm{MeV}$

$\mathrm{E}=7-9 \mathrm{MeV}$

$\mathrm{E}=8-20 \mathrm{MeV}$

$\mathrm{E}=6-27 \mathrm{MeV}$

$E=10-100 \mathrm{MeV}$

$E=6-27 \mathrm{MeV}$

$E=8-20 \mathrm{MeV}$

$E=10-120 \mathrm{MeV}$

$\mathrm{E}=10-120 \mathrm{MeV}$

$E=7-9 \mathrm{MeV}$

$E=10-120 \mathrm{MeV}$

$E=10-120 \mathrm{MeV}$

$\mathrm{E}=60 \mathrm{Me} \mathrm{V}$

$\mathrm{E}=1.3-2 \mathrm{MeV}$

$E=1.3-2 \mathrm{MeV}$

$E=1.3-2 \mathrm{MeV}$

$E=7-9$ MeV

$\mathrm{E}=1.3-2 \mathrm{MeV}$

$\mathrm{E}=1.3-2 \mathrm{MeV}$

$E=7-9 \mathrm{MeV}$

$\mathrm{E}=1.3-2 \mathrm{MeV}$

$E=10-100 \mathrm{MeV}$

$E=10-120 \mathrm{MeV}$

$E=7-9 \mathrm{MeV}$

$E=7-9 \mathrm{MeV}$

$E=7-9 \mathrm{MeV}$

$E=10-100 \mathrm{MeV}$

$E=10-100 \mathrm{MeV}$

$E=10-120 \mathrm{MeV}$

$E=60 \mathrm{MeV}$

$E \approx 4-90 \mathrm{MeV}$

$\mathrm{E}=7-9 \mathrm{MeV}$

$E \leq 50 \mathrm{MeV}$

$E=10-100 \mathrm{MeV}$

$E=10-100 \mathrm{MeV}$

$E=10-120 \mathrm{MeV}$

$E=60 \mathrm{MeV}$

$\mathrm{E} \approx 4-90 \mathrm{MeV}$

$E \approx 25-50 \mathrm{MeV}$

$E=10-100 \mathrm{MeV}$

$E=10-100 \mathrm{MeV}$

$E=10-120 \mathrm{MeV}$

$\mathrm{E}=60 \mathrm{MeV}$

$\mathrm{E} \approx 4-90 \mathrm{MeV}$

$\mathrm{E}=78 \mathrm{MeV}$
$\mathrm{E}=3-9 \mathrm{MeV}$

\section{REFERENCE}

JoUR Nucl.Instr.Meth. Phys.Res. B28, 199

KEY NUMBER

JolR Nucl.Instr.Meth. Phys.Res. B28, 199

JouR Nuclinstr.Meth. Phys.Res. B28, 199

B7Ra23

B7Ra23

B 7Raz3

JoUR Nucl.Instr.Meth. Phys.Res. B28, 199

$87 \operatorname{Ra} 23$

JOUR At.Energ. 62, 411

87 A 129

JoUR Nucl.Instr.Meth. Phys.Res. B24/25, 484

BTRAOB

JOUR Nucl.Instr.Meth. Phys.Res. B28, 199 87Raz3

JOUR Phys.Rev. C35, 2067

JOUR Phys.Rev. C35, 2067

JOUR At.Energ. 62, 411

JoUR Phys.Rev. C35, 2067

JOUR Phys.Rev. C35, 2077

JoUR J.Phys.(London) G13, 535

JouR Nucl.Instr.Meth. Phys.Res. B24/25, 484

JoUR Nucl.Instr.Meth. Phys.Res. B28, 199

87 We 05

87 We05

87 A 129

B7We 05

$87 \mathrm{Mu08}$

$87 \mathrm{RaO} 05$

87 Ra 08

JOUR J.Phys.(London) G13, 535

87Ra23

JouR Nucl.Instr.Meth. Phys.Res. B24/25, 484

JoUR Pramana 30, 193

JOUR Czech.J.Phys. B38, 150

JOUR Czech.J.Phys. B38, 741

87 RaO 05

$87 \mathrm{RaO} 0$

$881 \leq 01$

JouR Crech.J.Phys. B3B, 150

JoUR Nucl.Instr.Meth. Phys.Res. B28, 199

JOLR Czech.J.Phys. B38, 741

JOUR Czech.J.Phys. B38, 741

JOUR Nucl.Instr.Meth. Phys.Res, B28, 199

JouR Czech.J.Phys. B38, 150

885103

$885+14$

B8St03

$87 \mathrm{Ra} 33$

$88 \mathrm{St} 14$

885114

$87 \operatorname{Ra} 23$

$88 \mathrm{~S} 103$

JOUR At.Energ. 62. 411

JouR Nuclinstr.Meth. Phys.Res, B24/25, 484

87 A 129

JOUR Nucl.lnstr.Meth. Phys.Res. B28, 199

$87 \mathrm{Raz3}$

JOUR Yad.Fiz. 45, 614

B7Sk03

JoUR Nuclinstr.Meth. Phys.Res. B28, 199

B7Ra23

JoUR Nucl.Instr.Meth. Phys.Res. B28, 199 B7Ra23

JOUR At.Energ. 62, 411

JOUR At.Energ, 62,411

JoUR Nucl.Instr.Meth. Phys.Res, B24/25, 484

JOUR Pramana 30, 193

JoUR J.Radioanal.Nucl.Chem. 120, 49

87A 129

$87 \wedge 129$

$87 \mathrm{Ra} 08$

891501

$880 \times 67$

JOUR Nucl.Instr.Meth. Phys.Res. B28, 199 87Ra23

JOUR At.Energ. 62, $59 \quad 87 \mathrm{Ba} 61$

JOUR At.Energ. 62, 411

JoUR At.Energ. 62,411

87 A 129

87 A 129

JouR Nuclinstr.Meth. Phys.Res. B24/25, 484 g7Ra08

JoUR Pramana 30, 193

88 Is 01

JoUR J.Radioanal.Nucl.Chem. 120,49 88De07

JOUR At.Energ. 62, $59 \quad 87 \mathrm{Ba} 61$

JOUR At.Energ. 62, $411 \quad 87$ A129

JOUR At.Energ. 62, $411 \quad 87 \mathrm{~A} 129$

JoUR Nucl.Instr.Meth. Phys.Res. B24/25, $484 \quad 87 R a 08$

JOUR Pramana 30, 193

JOUR J.Radioanal.Nucl.Chem. 120, 49

881 s 01

88De0?

JOUR Yad.Fiz, 45, 1236

$87 \mathrm{G} 105$ 


\begin{tabular}{|c|c|c|c|c|c|}
\hline RESIDUAL & TARGET-REACTION & INC I DENT ENERGY & REFE & ERENCE & KEY NUMBER \\
\hline \multirow[t]{4}{*}{${ }^{60} \mathrm{Co}$} & ${ }^{5 \theta} \mathrm{Co}(\alpha, n 2 p)$ & $\mathrm{E}=10-120 \mathrm{MeV}$ & JOUR & Nucl.lnstr.Meth. Phys.Res. B24/25, 484 & $87 \mathrm{RaO} 08$ \\
\hline & ${ }^{59} \operatorname{Co}(\alpha, n 2 p)$ & $E=60 \mathrm{MeV}$ & JOUR & Pramana 30,193 & 88 I $\mathrm{s}_{1}$ \\
\hline & $\mathrm{Fe}(\alpha, x \cap p)$ & $\approx 4-90 \mathrm{MeV}$ & JOUR & J.Radioanal.Nucl.Chem. 120,49 & 88De07 \\
\hline & ${ }^{60} \mathrm{Ni}\left({ }^{7} \mathrm{Li},{ }^{7} \mathrm{Be}\right)$ & $\mathrm{E}=78 \mathrm{MeV}$ & JOUR & Yad.Fiz. 45,1236 & $87 \mathrm{G} 105$ \\
\hline${ }^{62} \mathrm{Co}$ & ${ }^{62} \mathrm{Ni}\left({ }^{7} \mathrm{Li},{ }^{7} \mathrm{Be}\right)$ & $\mathrm{E}=78 \quad \mathrm{MeV}$ & JOUR & Yad.Fiz. 45. 1236 & $87 \mathrm{G} 105$ \\
\hline${ }^{64} \mathrm{Co}$ & ${ }^{6+} \mathrm{Ni}\left({ }^{7} \mathrm{Li},{ }^{7} \mathrm{Be}\right)$ & $\mathrm{E}=78 \mathrm{MeV}$ & JOUR & Yad.Fiz. 45, 1236 & 870105 \\
\hline \multirow[t]{2}{*}{${ }^{58} \mathrm{Ni}$} & $\mathrm{Ni}(\mathbf{p}, \mathrm{X})$ & $E=10-100 \mathrm{MeV}$ & JoUR & At.Energ. 62,411 & $87 \mathrm{~A} / 29$ \\
\hline & ${ }^{39} \mathrm{Co}(\alpha, 6 \mathrm{np})$ & $E=10-120 \mathrm{MeV}$ & JOUR & Nucl.Instr.Meth. Phys.Res. B24/25, 484 & $87 R a 08$ \\
\hline \multirow[t]{5}{*}{${ }^{57} \mathrm{Ni}$} & $\mathrm{Ni}(\mathbf{p}, \mathrm{X})$ & $\mathrm{E}=10-100 \mathrm{MeV}$ & JOUR & At.Energ. 62,411 & $87 \times 129$ \\
\hline & ${ }^{58} \mathrm{Co}(\alpha, 5 \mathrm{np})$ & $E=10-120 \mathrm{MeV}$ & JOUR & Nucl.Instr.Meth. Phys.Res. B24/25, 484 & $87 \mathrm{RaOB}$ \\
\hline & $\mathrm{Ni}(\boldsymbol{a}, \boldsymbol{x} \cap \mathrm{y} \mathbf{p})$ & $\mathrm{E}=25-60 \mathrm{MeV}$ & JOUR & Nucl.Instr.Meth. Phys.Res. A263, 421 & $88 \mathrm{ChO} 1$ \\
\hline & ${ }^{58} \mathrm{Ni}\left({ }^{28} \mathrm{Si},{ }^{28} \mathrm{Si}\right)$ & $E(c m)=47-67 \mathrm{MeV}$ & JOUR & Phys.Rev. C 35,597 & $87 \mathrm{LaO} 04$ \\
\hline & ${ }^{58} \mathrm{Ni}\left({ }^{28} \mathrm{Si}, \mathrm{X}\right)$ & $E(c m)=47-67 \quad M e V$ & JOUR & Phys.Rev. C35, 597 & $87 \mathrm{LaO} 04$ \\
\hline \multirow[t]{4}{*}{${ }^{58} \mathrm{Ni}$} & ${ }^{58} \mathrm{Ni}\left({ }^{32} \mathrm{~S},{ }^{32} \mathrm{~S}\right)$ & $E=94-112 \mathrm{MeV}$ & JouR & Phys.Lett. 185,15 & $875 t 06$ \\
\hline & ${ }^{58} \mathrm{Ni}\left({ }^{32} \mathrm{~S},{ }^{32} \mathrm{~S}^{\prime}\right)$ & $\mathrm{E}=94-112 \mathrm{MeV}$ & JOUR & Phys.tett 185, 15 & $875+06$ \\
\hline & ${ }^{58} \mathrm{Ni}\left({ }^{36} \mathrm{~S},{ }^{36} \mathrm{~S}\right)$ & $\mathrm{E}=94-112 \mathrm{MeV}$ & JOUR & Phys.Lett. 185, 15 & $875 t 06$ \\
\hline & ${ }^{58} \mathrm{~N}:\left({ }^{36} \mathrm{~S},{ }^{36} \mathrm{~S}^{\prime}\right)$ & $\mathrm{E}=94-112 \mathrm{MeV}$ & JOUA & Phys.Lett. 185,15 & $87 \mathrm{St06}$ \\
\hline${ }^{59} \mathrm{Ni}$ & ${ }^{59} \mathrm{Co}(p, \Omega)$ & $\mathrm{E}=7-9 \mathrm{MeV}$ & JOUR & Nucl.Instr.Meth. Phys.Res. B28, 199 & $87 R \mathbf{R} 3$ \\
\hline${ }^{60} \mathrm{Ni}$ & ${ }^{58} \mathrm{Co}(\mathrm{p}, \gamma)$ & $\mathrm{E}=7-9 \mathrm{MeV}$ & JOUR & Nucl.Instr.Meth. Phys.Res. B28, 199 & $87 \operatorname{Ra} 23$ \\
\hline \multirow[t]{3}{*}{${ }^{61} \mathrm{Ni}$} & ${ }^{61} \mathrm{Ni}\left(\mathbf{P}, \mathrm{P}^{\prime}\right)$ & $\mathrm{E}=3.08-4.66 \mathrm{MeV}$ & JOUR & Nucl.Phys. A480, 162 & $88 \mathrm{Ti03}$ \\
\hline & ${ }^{62} \mathrm{Ni}\left({ }^{28} \mathrm{Si},{ }^{29} \mathrm{Si}\right)$ & $\mathrm{E}(\mathrm{cm})=47-67 \mathrm{MeV}$ & JOUR & Phys.Rev. C35, 597 & $87 \mathrm{LaO} 4$ \\
\hline & ${ }^{62} \mathrm{Ni}\left({ }^{2 \mathrm{~B}} \mathrm{~S}_{1}, \mathrm{X}\right)$ & $E(c m)=47-67 \mathrm{MeV}$ & JOUR & Phys.Rev. C35, 597 & $87 \mathrm{LaO} 4$ \\
\hline${ }^{62} \mathrm{Ni}$ & ${ }^{59} \mathrm{Co}(\alpha, p)$ & $E=5.07-9.8 \mathrm{MeV}$ & JOUR & Nucl.Phys. A483, 354 & $88 \mathrm{Ti} 05$ \\
\hline \multirow[t]{2}{*}{${ }^{63} \mathrm{Ni}$} & ${ }^{64} \mathrm{Ni}\left({ }^{28} \mathrm{Si},{ }^{29} \mathrm{Si}\right)$ & $E(c m)=47-67 \mathrm{MeV}$ & JOUR & Phys.Rev. C35, 597 & $87 \mathrm{LaO} 4$ \\
\hline & ${ }^{64} \mathrm{Ni}\left({ }^{28} \mathrm{Si}, \mathrm{X}\right)$ & $\mathrm{E}(\mathrm{cm})=47-67 \mathrm{MeV}$ & JOUR & Phys.Rev. C35, 597 & $87 \mathrm{LaO4}$ \\
\hline \multirow[t]{4}{*}{${ }^{64} \mathrm{Ni}$} & $04 \mathrm{Ni}\left({ }^{32} \mathrm{~S},{ }^{32} \mathrm{~S}\right)$ & $\mathrm{E}=94-112 \mathrm{MeV}$ & JOUR & Phys.Lett. 185,15 & $875 t 06$ \\
\hline & ${ }^{64} \mathrm{Ni}\left({ }^{32} \mathrm{~S},{ }^{32} \mathrm{~S}^{\prime}\right)$ & $\mathrm{E}=94-112 \mathrm{MeV}$ & JOUR & Phys.Lett, 185, 15 & $875 t 06$ \\
\hline & ${ }^{64} \mathrm{Ni}\left({ }^{36} \mathrm{~S},{ }^{36} \mathrm{~S}\right)$ & $E=94-112 \mathrm{MeV}$ & JOUR & Phys.Lett. 185, 15 & $87 S t 06$ \\
\hline & ${ }^{84} \mathrm{Ni}\left({ }^{38} \mathrm{~S},{ }^{38} \mathrm{~S}^{\prime}\right)$ & $\mathrm{E}=94-112 \mathrm{MeV}$ & JOUR & Phys.Lett. 185. 15 & $875 t 06$ \\
\hline${ }^{58} \mathrm{Cu}$ & ${ }^{58} \mathrm{Ni}(p, n)$ & $\mathrm{E}=7-9 \mathrm{MeV}$ & JOUR & Nucl.Instr.Meth. Phys.Res. B28, 199 & $87 R a 23$ \\
\hline \multirow[t]{3}{*}{${ }^{90} \mathrm{Cu}$} & ${ }^{90} \mathrm{Ni}(\mathrm{p}, \gamma)$ & $\mathrm{E}=7-9 \mathrm{MeV}$ & JOUR & Nucl.Instr.Meth. Phys.Res, B28, 199 & $87 \mathrm{Ra} 23$ \\
\hline & ${ }^{58} \mathrm{Ni}\left({ }^{20} \mathrm{Si},{ }^{27} \mathrm{Al}\right)$ & $\mathrm{E}(\mathrm{cm})=47-67 \mathrm{MeV}$ & JOUR & Phys.Rev. C35. 597 & $87 \mathrm{LaO}_{4}$ \\
\hline & ${ }^{58} \mathrm{Ni}\left({ }^{30} \mathrm{Si}, \mathrm{X}\right)$ & $E(c m)=4 \gamma-6 \gamma \quad \mathrm{MeV}$ & JOUR & Phys.Rev. C35, 597 & B7LaO4 \\
\hline \multirow[t]{4}{*}{${ }^{60} \mathrm{Cu}$} & ${ }^{00} \mathrm{Ni}(p, n)$ & $\mathrm{E}=7-9 \mathrm{MeV}$ & JOUR & Nucl.Instr.Meth. Phys.Res. B28, 199 & $87 \operatorname{Ra} 23$ \\
\hline & $\mathrm{Ni}(p, \mathrm{X})$ & $\mathrm{E}=10-100 \mathrm{MeV}$ & JOUR & At.Energ. 62.411 & B7A129 \\
\hline & $\mathrm{Cu}(\mathrm{p}, \mathrm{X})$ & $E=10-100 \mathrm{MeV}$ & JOUR & At.Energ. 62,411 & $87 A 129$ \\
\hline & $N i(a, x n y p)$ & $E=25-60 \mathrm{MeV}$ & JOUR & Nucl.Instr.Meth. Phys.Res. A263, 421 & $88 \mathrm{ChO} 1$ \\
\hline \multirow[t]{5}{*}{${ }^{61} \mathrm{Cu}$} & ${ }^{60} \mathrm{Ni}(p, \gamma)$ & $\mathrm{E}=7-9 \mathrm{MeV}$ & JOUR & Nucl.Instr.Meth. Phys.Res. B28, 199 & $87 \operatorname{Ra} 33$ \\
\hline & ${ }^{1} \mathrm{Ni}(p, n)$ & $\mathbf{E}=1.05-3.98 \mathrm{MeV}$ & JOUR & Nucl.Phys. A480, 162 & $88 \mathrm{~T}$ i 03 \\
\hline & $\operatorname{Cu}(p, x)$ & $E=10-100 \mathrm{MeV}$ & JOUR & A.t.Energ. 62, 411 & $B 7 A 129$ \\
\hline & ${ }^{50} \mathrm{Ni}(\alpha, p)$ & $\mathrm{E}=4-30 \mathrm{MeV}$ & JOUR & Rep.Prog.Phys. 50, 1171 & 87 H०22 \\
\hline & $\mathrm{Ni}(\alpha, x \cap y p)$ & $\mathrm{E}=25-60 \mathrm{MeV}$ & JOUR & Nucl.Instr.Meth. Phys.Res. A263. 421 & $88 \mathrm{Ch} 01$ \\
\hline \multirow[t]{2}{*}{${ }^{62} \mathrm{Cu}$} & ${ }^{\theta 1} \mathrm{Ni}(\mathrm{p}, \gamma)$ & $\mathrm{E}=1.05-3.98 \mathrm{MeV}$ & JOUR & Nucl.Phys. A480, 162 & $88 \mathrm{Ti03}$ \\
\hline & ${ }^{s e} \mathrm{Co}(a, n)$ & $\mathrm{E}=5.1 \mathrm{~B}-9.4 \mathrm{MeV}$ & JOUR & Nucl.Phys. A483, 354 & $88 \mathrm{~T}$ i 05 \\
\hline \multirow[t]{2}{*}{${ }^{63} \mathrm{Cu}$} & ${ }^{62} \mathrm{Ni}\left({ }^{20} \mathrm{Sj}_{1}{ }^{27} \mathrm{Al}\right)$ & $\mathrm{E}(\mathrm{cm})=47-67 \mathrm{MeV}$ & JOUR & Phys.Rev. C35. 597 & 87 La 04 \\
\hline & ${ }^{82} \mathrm{Ni}\left({ }^{30} \mathrm{Si}, \mathrm{X}\right)$ & $\mathrm{E}(\mathrm{cm})=47-67 \mathrm{MeV}$ & JOUR & Phys.Rev. C35, 597 & $87 \mathrm{LaO} 4$ \\
\hline \multirow[t]{2}{*}{${ }^{84} \mathrm{Cu}$} & ${ }^{84} \mathrm{Ni}(p, n)$ & $\mathrm{E}=4-30 \mathrm{MeV}$ & JOUR & Rep.Prog.Phys. 50, 1171 & 87Ho22 \\
\hline & $\operatorname{cu}(a, x)$ & $\mathrm{E} \leq 40 \mathrm{MeV}$ & JOUR & A ppl.Radiat.Isot. 38,499 & $872 * 01$ \\
\hline
\end{tabular}


RESIDUAL TARGE'T-REACTION INCIDENT ENERGY

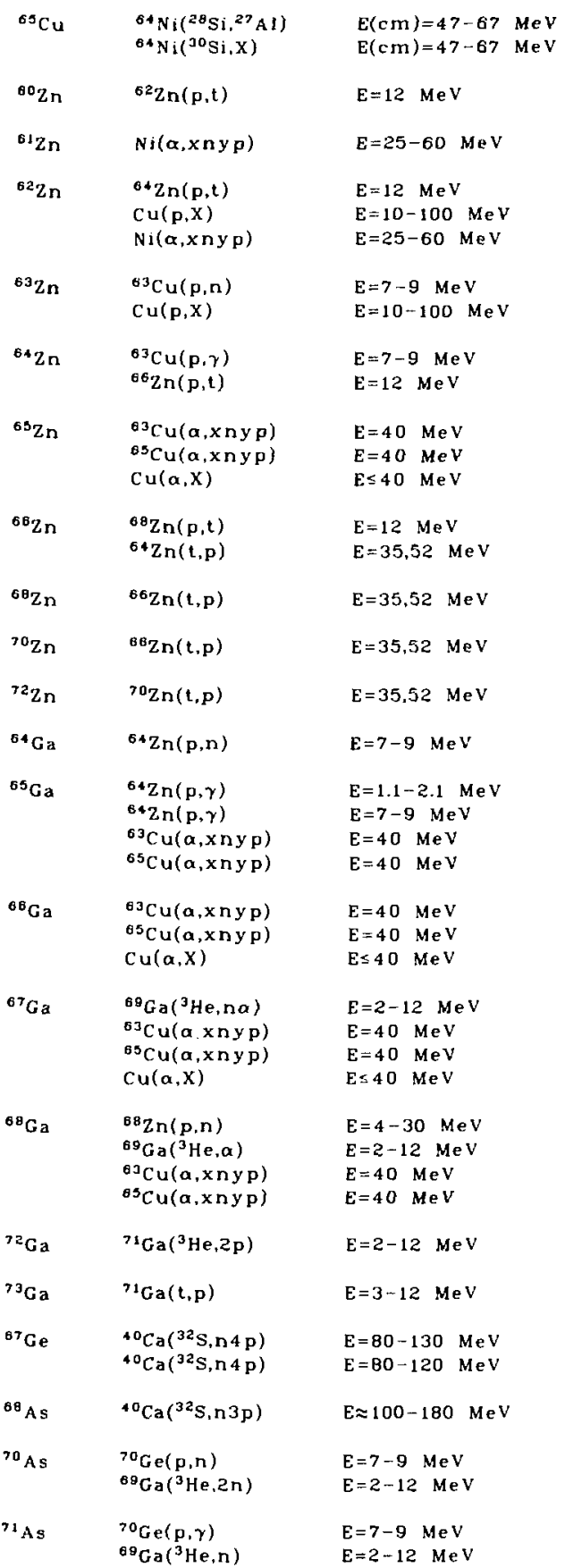

\section{REFERENCE}

JOUR Phys.Rev. C35, 597

JOUR Phys.Rev. C35, 597

JOUR Phys.Rev, C37, 556

JoUR Nucl.Instr.Meth. Phys.Res. A263, 421

JOUR Phys.Rev. C37, 556

JOUR At.Energ. 62, 411

JOUR Nucl.Instr.Meth. Phys.Res. A263, 421

JOUR Nucl.Instr.Meth. Phys.Res. B28, 199

JOUR At.Energ. 62,411

JoUR Nucl.Instr.Meth. Phys.Res. B28, 199 JOUR Phys.Rev. C37, 556

JOUR J.Phys.Soc.Jpn, 56, 3135

JOUR J.Phys.Soc.Jpn. 56, 3135

JOUR Appl.Radiat.Isot. 38, 499

JoUR Phys.Rev. C37. 556

JOUR Phys.Rev. C37, 556

JouR Phys.Rev. C37, 556

JOUR Phys.Rev. C37, 556

JOUR Phys.Rev. C37, 556

JoUR Nucl.Instr.Meth. Phys.Res. B28, 199

JoUR Nucl.Phys, A 463, 605

JoUR Nucl.Instr.Meth. Phys.Res. B2B, 199

jOUR J.Phys.Soc.Jpn. 56, 3135

JOUR J.Phys.Soc.Jpn. 56, 3135

JOUR J.Phys.Soc.Jpn. 56, 3135

JOUR J.Phys.Soc.Jpn. 56, 3135

JOUR Appl.Radiat.Isot. 38, 499

JoUR Nucl.Instr.Meth. Phys.Res. B18, 281

JOUR J.Phys.Soc.Jpn. 56, 3135

JOUR J.Phys.Soc.Jpn. 56, 3135

JOUR Appl.Radiat.Isol. 38, 499

JOUR Rep.Prog.Phys. 50, 1171

JoUR Nucl.Instr.Meth. Phys.Res. B18, 2B1

JOUR J.Phys.Soc.Jpn. 56, 3135

JOUR J.Phys.Soc.Jpn. 56, 3135

Jour Nucl.Instr.Meth. Phys.Res. B18, 281

JoUR Nucl.Instr.Meth. Phys.Res. B24/25, 722

JOUR Phys.Rev, C37, 645

JOUR Phys.Rev. C37, 645

JOUR Phys.Rev, C37, 645

JouR Nucl.Instr.Meth. Phys.Res. B28, 199

JoUR Nucl.lnstr.Meth. Phys.Res. B18, 281

JOUR Nucl.Instr.Meth. Phys.Res. B28, 199

JouR Nucl.Instr.Meth. Phys.Res. B18, 281
KEY NUMBEK

$87 \mathrm{La} 04$

$87 \mathrm{LaO}_{4}$

$88 \mathrm{CaO} 4$

$88 \mathrm{ChO}$

$8 B \mathrm{CaO} 4$

87A129

$\mathrm{BBChO} 1$

$87 R$ a23

$87 \mathrm{~A} 12 \mathrm{~S}$

$87 \mathrm{Ra} 23$

$88 \mathrm{Ca} 04$

87Ri 09

87Ri09

872W01

$88 \mathrm{Ca} 04$

$88 \mathrm{CaO} 4$

$88 \mathrm{Ca} 04$

$88 \mathrm{C}$ य 04

$88 \mathrm{Ca} 04$

$87 \operatorname{Ra} 23$

$87 \mathrm{Vi} 01$

$87 \mathrm{Ra} 23$

87Ri 09

87Ri 09

87 K 09

87R i 09

B7ZW01

$87 \mathrm{Mi0} 05$

87Ri09

87Ri 09

87Z 11

$87 \mathrm{Ho} 22$

87Mi0 5

87R॥ 09

87Ri 09

87M105

$87 \operatorname{Bo1} 6$

88Ra06

88Ra06

B8RaOb

87Ra23

$87 \mathrm{Mi} 05$

87Ra23

87M:05 


\begin{tabular}{|c|c|c|c|c|c|}
\hline RES IDUAL & TARGET - REACT ION & INCIDENT ENERGY & REFE & RENCE & KEY NUMBE \\
\hline${ }^{22} \mathrm{AS}$ & ${ }^{72} \mathrm{Ge}(\mathrm{p}, \mathrm{n})$ & $E=7-9 \mathrm{MeV}$ & JOUR & Nuclinstr. Meth. Phys.Res. B28, 199 & 87 คа23 \\
\hline${ }^{33} \mathrm{As}$ & ${ }^{72} \mathrm{Ge}(p, \gamma)$ & $\mathrm{E}=7-9 \mathrm{MeV}$ & JOUR & Nucl.Instr.Meth. Phys.Res. B28, 199 & 87 Ra23 \\
\hline${ }^{70} \mathrm{As}$ & ${ }^{35} \mathrm{~A} s(\mathrm{c}, \mathrm{d})$ & $\mathbf{E}=3-12 \mathrm{MeV}$ & JOUR & Nucl.Instr.Meth. Phys.Res. B24/25, 722 & 878016 \\
\hline${ }^{72} \mathrm{Se}$ & ${ }^{r a} \mathrm{Ge}(\alpha, 2 \mathrm{2n})$ & $E=$ threshold -24 MeV & JOUR & Rev.Roum.Phys. 32, 725 & 87 .a33 \\
\hline \multirow[t]{4}{*}{${ }^{73} \mathrm{Se}$} & ${ }^{75} \mathrm{As}(p, 3 n)$ & $E=25-55 \mathrm{MeV}$ & JOUR & Phys.Rev C38, 645 & $88 Q 902$ \\
\hline & ${ }^{73} \mathrm{As}(d, 4 n)$ & $E=13-27 \quad \mathrm{MeV}$ & JOUR & Phys.Rev. C38, 645 & B8Qa02 \\
\hline & ${ }^{70} \mathrm{Ge}(\alpha, n)$ & $\mathrm{E}=$ threshold $-24 \mathrm{MeV}$ & JOUR & Rev.Roum.Phys. 32, 725 & $87 \mathrm{Ca} 33$ \\
\hline & ${ }^{70} \mathrm{Ge}(\alpha, n)$ & $\mathrm{E}=13-24 \mathrm{MeV}$ & JOUR & Phys.Rev. C38, 645 & $88 Q \mathrm{aO} 2$ \\
\hline${ }^{25} \mathrm{Br}$ & ${ }^{7 \theta_{K}} \mathrm{r}(d, n \alpha)$ & $\mathrm{E}=21.5 \mathrm{MeV}$ & JOUR & Appl.Radiat.Isot. 39,709 & $88 D e 24$ \\
\hline${ }^{76} \mathrm{Br}$ & ${ }^{75} \mathrm{As}\left({ }^{3} \mathrm{He}, 2 \mathrm{n}\right)$ & $E=2-12 \mathrm{MeV}$ & JOUR & Nuclinstr.Meth. Phys.Res. B18, 281 & $87 \mathrm{MiO} 05$ \\
\hline \multirow[t]{2}{*}{${ }^{77} \mathrm{Br}$} & ${ }^{77} \mathrm{Se}(\mathrm{p}, \mathrm{n})$ & $\mathrm{E} \approx 2.8-3.5 \mathrm{MeV}$ & JOUR & Yadfiz. 48,12 & $88 \mathrm{Fe} 07$ \\
\hline & ${ }^{75} \mathrm{As}\left({ }^{3} \mathrm{He}, \mathrm{n}\right)$ & $\mathrm{E}=2-12 \mathrm{MeV}$ & JOUR & Nucl.Jnstr.Meth. Phys.Res. B18, 281 & $87 M+05$ \\
\hline \multirow[t]{6}{*}{${ }^{8} \mathrm{R} b$} & ${ }^{82} K r(p, 2 n)$ & \multirow{2}{*}{$\begin{array}{l}E \approx \text { threshold-.0 MeV } \\
E \approx \text { threshol } r_{1}-40 \mathrm{MeV}\end{array}$} & JOUR & Radiochim.Acta 41,111 & B7Qa 02 \\
\hline & ${ }^{85} R b(p, 4 n p)$ & & JOUR & Radiochim.Acta 41,111 & B7Qa 02 \\
\hline & ${ }^{B_{1}} \mathrm{Br}\left({ }^{3} \mathrm{He}, 3 \mathrm{n}\right)$ & $E \approx 50-70 \mathrm{Me}$ & JOUK & Radiochim.Acta 41,111 & 87Qa 02 \\
\hline & $\mathrm{Kr}\left({ }^{3} \mathrm{He}, \mathrm{x} \cap \mathrm{p}\right)$ & $\mathrm{E}=10-25 \mathrm{MeV}$ & JOUR & Appl.Radiat.isot. 39,135 & $88 \mathrm{Ta} 02$ \\
\hline & ${ }^{79} \mathrm{Br}(\alpha, 2 \mathrm{n})$ & $E \approx$ threshold $-40 \mathrm{MeV}$ & JOUR & Radiochim.Acta 41, 111 & $87 Q \mathrm{a} 02$ \\
\hline & $\mathrm{K} \mathbf{r}(\boldsymbol{a}, \mathrm{x} \mathbf{n} \mathbf{p})$ & $\mathrm{E}=10-33 \mathrm{MeV}$ & JOUR & Appl.Radiat.Isot. 39,135 & $88 \mathrm{TaO} 02$ \\
\hline \multirow[t]{2}{*}{${ }^{82} \mathrm{Rb}$} & $\mathrm{Kr}\left({ }^{3} \mathrm{He}, \mathrm{x} n \mathrm{p}\right)$ & $\mathrm{E}=10-25 \mathrm{MeV}$ & JouR & Appl.Radiat.Isot. 39, 135 & $88 \mathrm{TaO}$ \\
\hline & $\operatorname{Kr}(\alpha, x \cap p)$ & $\mathrm{E}=10-33 \mathrm{MeV}$ & JOUR & Appl.Radiat.Isot. 39,135 & $88 \mathrm{TaO} 2$ \\
\hline${ }^{83} \mathrm{Rb}$ & $\mathrm{Kr}\left({ }^{3} \mathrm{He}, \mathrm{xnp}\right)$ & $\mathrm{E}=10-25 \mathrm{MeV}$ & JOUR & Appl.Radiat.]sot. 39,135 & BETa02 \\
\hline & $\operatorname{Kr}(a, x \cap p)$ & $E=10-33 \mathrm{MeV}$ & JOUR & Appl.Radiat.jsot, 39,135 & $88 \mathrm{Ta} 02$ \\
\hline${ }^{84} \mathrm{Rb}$ & $\mathrm{Kr}\left({ }^{3} \mathrm{He}, \mathrm{xnp}\right)$ & $\mathrm{E}=10-25 \mathrm{MeV}$ & JOUR & A ppl.Radiat.Isot. 39,135 & B8Ta 02 \\
\hline & ${ }^{85} \mathrm{Rb}(\alpha, \mathrm{n} \alpha)$ & $E=20-60 \mathrm{MeV}$ & JOUR & J.Radioanal.Nucl.Chem. 119,303 & $87 \mathrm{Sa} 49$ \\
\hline & $\mathrm{Kr}(\alpha, \mathbf{x n p})$ & $E=10-33 \mathrm{MeV}$ & JOUR & Appl.Radiat.Isot. 39,135 & 88Та 02 \\
\hline${ }^{86} \mathrm{Rb}$ & $\mathrm{Kr}\left({ }^{3} \mathrm{He}, \mathrm{x} \cap \mathrm{p}\right)$ & $E=10-25 \mathrm{MeV}$ & JOUR & Appl.Radiat.lsot. 39,135 & $88 \mathrm{Ta} 02$ \\
\hline & $\mathrm{K} r(\alpha, x \cap p)$ & $E=10-33 \mathrm{MeV}$ & JOUR & Appl.Radiat.Isot. 39,135 & $88 \mathrm{Ta02}$ \\
\hline${ }^{82} \mathrm{Sr}$ & $\mathrm{Kr}\left({ }^{3} \mathrm{He}, \times \mathrm{n}\right)$ & $E \approx 10-35 \mathrm{MeV}$ & JouR & Appl.Radiat.Isot. 39,135 & $88 \mathrm{Ta} 02$ \\
\hline & $\mathrm{Kr}\left({ }^{3} \mathrm{He}, \mathrm{xn}\right)$ & $E=10-33 \mathrm{MeV}$ & JOUR & Appl.Radiat.Isot. 39,135 & 88Ta02 \\
\hline & $\operatorname{Kr}(\alpha, x n)$ & $E=10-25 \mathrm{MeV}$ & JOUR & Appl.Radiat.Isot. 39,135 & $88 \mathrm{TaO} 02$ \\
\hline${ }^{8} \mathrm{Sr}$ & $\mathrm{Kr}\left({ }^{3} \mathrm{He}, \mathrm{xn}\right)$ & $E \approx 10-35 \mathrm{MeV}$ & JOUR & Appl.Radiat.1sot. 39,135 & $88 \mathrm{TaO} 02$ \\
\hline & $\mathrm{Kr}\left({ }^{3} \mathrm{He}, \mathrm{xn}\right)$ & $E=10-33 \mathrm{MeV}$ & JOUR & Appl.Radjat.Isot. 39,135 & $88 \mathrm{Ta} 02$ \\
\hline & $\operatorname{Kr}(\boldsymbol{a}, \mathbf{x n})$ & $E=10-25 \mathrm{MeV}$ & JOUR & Appl.Radiat.Isot. $39, \quad 135$ & B8Ta02 \\
\hline${ }^{85} \mathrm{Sr}$ & $\mathrm{Kr}\left({ }^{3} \mathrm{He}, \mathrm{Xn}\right)$ & $\mathrm{E} \approx 10-35 \mathrm{MeV}$ & JOUR & Appl.Radiat.Isot. 39,135 & B8Ta 02 \\
\hline & $\mathrm{Kr}\left({ }^{3} \mathrm{He}, \mathrm{xn}\right)$ & $E=10-33 \mathrm{MeV}$ & JOUR & Appl.Radiat.1sot. 39,135 & B8Ta02 \\
\hline & $\operatorname{Kr}(\alpha, x n)$ & $E=10-25 \mathrm{MeV}$ & JOUR & Appl-Radiat.1sot. 39,135 & $88 T a 02$ \\
\hline${ }^{87} \mathrm{Sr}$ & $\mathrm{Kr}\left({ }^{3} \mathrm{He}, \mathrm{xn}\right)$ & $E=10-33 \mathrm{MeV}$ & JOUR & Appl.Radiat.lsot. 39,135 & $88 \mathrm{Ta} 02$ \\
\hline & $\mathrm{Kr}(\alpha, \times n)$ & $E=10-25 \mathrm{MeV}$ & JOUR & Appl.Radiat.lsol. 39,135 & 88Ta 02 \\
\hline${ }^{80} \mathrm{Y}$ & ${ }^{85} \mathrm{Rb}(\alpha .3 n)$ & $E=20-60 \mathrm{MeV}$ & JOUR & J.Radioanal.Nucl.Chem. 119, 303 & $875 a 49$ \\
\hline${ }^{87} \mathrm{Y}$ & ${ }^{85} \mathrm{Rb}(a, 2 \mathrm{n})$ & $E=20-60 \mathrm{MeV}$ & JOUR & J.Radioanal.Nucl.Chem. 119, 303 & $875 a 49$ \\
\hline & $\operatorname{Zr}(\alpha, X)$ & $E=11,13,21-22.6 \mathrm{MeV}$ & JOUR & At.Energ. 60,332 & $86 K_{0} 33$ \\
\hline & $\operatorname{zr}(\alpha, x)$ & $E=6.1-22.4 \mathrm{MeV}$ & JOUR & At.Energ. 60,332 & $86 \mathrm{~K} 033$ \\
\hline & $N \mathbf{b}(a, X)$ & $E=6.1-22.4 \mathrm{MeV}$ & JOUR & At.Energ. 60,332 & $86 K 033$ \\
\hline & $\mathrm{Nb}(\alpha, \mathrm{X})$ & $E=11,13,21-22.6 \mathrm{MeV}$ & JOUR & At.Energ. 60,332 & $86 \mathrm{Ko33}$ \\
\hline & $\operatorname{Ta}(\alpha, \mathrm{X})$ & $E=6.1-22.4 \mathrm{MeV}$ & JOUR & A t.Energ. 60,332 & $86 \mathrm{Ko33}$ \\
\hline & $\operatorname{Ta}(\alpha, X)$ & $E=11,13,21-22.6 \mathrm{MeV}$ & JOUR & A t.Energ. 60,332 & $86 \mathrm{Ko33}$ \\
\hline
\end{tabular}


${ }^{\text {ae }} Y$

${ }^{\theta B} \mathrm{Sr}(p, n)$

${ }^{8} \mathrm{Y}(\mathrm{p}, \mathrm{np})$

${ }^{87} \operatorname{Sr}(d, n)$

${ }^{87} \mathrm{Rb}\left({ }^{3} \mathrm{He}, 2 \mathrm{n}\right)$

${ }^{85} \mathrm{R} b(\alpha, n)$

$\operatorname{Zr}(\alpha, X)$

$\operatorname{Zr}(\alpha, X)$

$N b(\alpha, X)$

$N b(a, x)$

$\operatorname{Ta}(\alpha, X)$

$\operatorname{Ta}(\alpha, X)$

\begin{tabular}{|c|c|}
\hline${ }^{80} \mathrm{Y}$ & ${ }^{90} \mathrm{Zr}\left({ }^{7} \mathrm{Li},{ }^{7} \mathrm{Be}\right)$ \\
\hline $92 Y$ & ${ }^{92} \mathrm{Zr}\left({ }^{7} \mathrm{Li},{ }^{7} \mathrm{Be}\right)$ \\
\hline $94 Y$ & ${ }^{84} \mathrm{Zr}\left({ }^{7} \mathrm{Li},{ }^{7} \mathrm{Be}\right)$ \\
\hline${ }^{8 \theta} \mathrm{Zr}$ & ${ }^{89} \mathrm{Y}(\mathrm{p}, 2 \mathrm{n})$ \\
\hline${ }^{89} \mathrm{Zr}$ & $\begin{array}{l}{ }^{89} Y(p, n) \\
{ }^{89} Y(p, n) \\
{ }^{89} Y(d, 2 n) \\
\operatorname{Zr}(a, X) \\
\operatorname{Zr}(a, X) \\
\operatorname{N} b(a, X) \\
\operatorname{N~} b(a, X) \\
\operatorname{Ta}(a, X) \\
\operatorname{Ta}(a, X)\end{array}$ \\
\hline${ }^{80} \mathrm{Zr}$ & $\begin{array}{l}{ }^{8} \mathrm{Y}(p, \gamma) \\
{ }^{80} \mathrm{Zr}(p, p) \\
{ }^{80} \mathrm{Zr}\left(p, p^{\prime}\right)\end{array}$ \\
\hline
\end{tabular}

${ }^{95} \mathrm{Zr}_{\mathrm{r}} \quad \mathrm{Zr}(a, X)$

$\operatorname{Zr}(a, X)$

$\mathrm{Nb}(\alpha, x)$

$\mathrm{Nb}(\alpha, X)$

$\operatorname{Ta}(\alpha, x)$

$\operatorname{Ta}(\alpha, x)$

$\begin{array}{ll}{ }^{80} \mathrm{Nb} & { }^{90} \mathrm{Zr}(p, n) \\ & { }^{90} \mathrm{Zr}(p, n) \\ & { }^{83} \mathrm{Nb}(\alpha, x n 2 p) \\ & { }^{93} \mathrm{Nb}(\alpha, x n \alpha)\end{array}$

${ }^{0} \mathrm{Nb}$

${ }^{90}{ }_{2 r}(p, \gamma)$

${ }^{90} \mathrm{Z} \mathbf{r}(\mathrm{p}, \gamma)$

${ }^{\theta 1} \mathrm{Zr}(\mathrm{p}, \mathrm{n})$

${ }^{83} \mathrm{Nb}(\alpha, x \mathrm{n} 2 \mathrm{p})$

${ }^{93} \mathrm{Nb}(\alpha, x \cap a)$

$\operatorname{Zr}(\alpha, x)$

$\operatorname{Zr}(\alpha, x)$

$\mathrm{Nb}(\alpha, \mathrm{X})$

$N b(\alpha, X)$

$\operatorname{Ta}(\alpha, X)$

$\operatorname{Ta}(\alpha, X)$

${ }^{92} \mathrm{Nb} \quad{ }^{92} \mathrm{Z}_{\mathrm{S}}(\mathrm{p}, \mathrm{n})$

${ }^{93} \mathrm{Nb}(a, x \mathrm{n} 2 \mathrm{p})$

${ }^{83} \mathrm{Nb}(a, x n \alpha)$

$\operatorname{Zr}(a, x)$

$\operatorname{Zr}(\alpha, x)$

$N b(a, x)$

$\mathrm{Nb}(a, \mathrm{X})$

$\mathrm{Ta}(\alpha, X)$
$E=7.3 \mathrm{MeV}$

$E=4.59-39.56 \mathrm{MeV}$

$E=19.3 \mathrm{MeV}$

$E=12.8 \mathrm{MeV}$

$E=16 \mathrm{MeV}$

$E=11,13,21-22.6 \mathrm{MeV}$

$E=6.1-22.4 \mathrm{MeV}$

$\mathrm{E}=11,13,21-22.6 \mathrm{MeV}$

$\mathrm{E}=6.1-22.4 \mathrm{MeV}$

$E=11,13,21-22.6 \mathrm{MeV}$

$\mathrm{E}=6.1-22.4 \mathrm{MeV}$

$\mathrm{E}=78 \mathrm{MeV}$

$E=78 \quad \mathrm{MeV}$

$\mathrm{E}=78 \mathrm{MeV}$

$E=4.59-39.56 \mathrm{KieV}$

$\mathrm{E}=4.59-39.56 \mathrm{MeV}$

$\mathrm{E}=7-9 \mathrm{MeV}$

$\mathrm{E}<13.5 \mathrm{MeV}$

$\mathrm{E}=11,13,21-22.6 \mathrm{MeV}$

$\mathrm{E}=6.1-22.4 \mathrm{MeV}$

$E=11,13,21-22.6 \mathrm{MeV}$

$\mathrm{E}=6.1-22.4 \mathrm{MeV}$

$E=11,13,21-22.6 \mathrm{MeV}$

$\mathrm{E}=6.1-22.4 \mathrm{MeV}$

$\mathrm{E}=7-9 \mathrm{MeV}$

$\mathrm{E}=3.9-5.7 \mathrm{MeV}$

$E=1.9-5.7 \mathrm{MeV}$

$E=11,13,21-22.6 \mathrm{MeV}$

$E=6.1-22.4 \mathrm{MeV}$

$E=6.1-22.4 \mathrm{MeV}$

$E=11,13,21-22.6 \mathrm{MeV}$

$\mathrm{E}=11,13,21-22.6 \mathrm{MeV}$

$E=6.1-22.4 \mathrm{MeV}$

E $\leq 9 \mathrm{MeV}$

$\mathrm{E}=7-9 \mathrm{MeV}$

$\mathrm{E} \leq 90 \mathrm{MeV}$

$\mathrm{E} \leq 90 \mathrm{MeV}$

$\mathrm{E}=7-9 \mathrm{MeV}$

$E=2-7$ MeV

$\mathrm{E} \approx 12-40 \mathrm{MeV}$

$\mathrm{E} \leq 90 \mathrm{MeV}$

$\mathrm{E} \leq 90 \mathrm{MeV}$

$\mathrm{E}=6.1-22.4 \mathrm{MeV}$

$E=11,13,21-22.6 \mathrm{MeV}$

$E=11,13,21-22.6 \mathrm{MeV}$

$E=6.1-22.4 \mathrm{MeV}$

$\mathrm{E}=11,13,21-22.6 \mathrm{MeV}$

$\mathrm{E}=6.1-22.4 \mathrm{MeV}$

Es9 MeV

Es $90 \mathrm{MeV}$

$\mathrm{E} s 90 \mathrm{MeV}$

$E=6.1-22.4 \mathrm{MeV}$

$\mathrm{E}=11,13,21-22.6 \mathrm{MeV}$

$\mathrm{E}=11,13,21-22.6 \mathrm{MeV}$

$\mathrm{E}=6.1-22.4 \mathrm{MeV}$

$E=11,13,21-22.6 \mathrm{MeV}$

\section{REFERENCE}

JOUR J.Radioanal.Nucl.Chem. 119, 433

JOUR Phys.Rev, C38, 1624

JOUR J.Radioanal.Nucl.Chem. 119, 433

JoUR J.Radioanal.Nucl.Chem. 119, 433

JUUR J.Radioanal.iucl.Chem. 119, 433

JoUR At.Energ. 60, 332

JOUR At.Energ. 60, 332

JOUR At.Energ. 60,332

JoUR At.Energ. 60, 332

JOUR At.Energ. 60, 332

JOUR At.Energ. 60,332

JOUR Yad.Fiz. 45, 1236

JOUR Yad.Fiz. 45, 1236

JOUR Yad.Fiz. 45,1236

JOUR Phys.Rev. C3a, 1624

JoUR Phys.Rey. C38. 1624

JUUR Nucl.Instr.Meth. Phys.Res. B28, 199

JUU'R J.Radioanal.Nuc].Chem. 127, 7

JoUR At.Energ. 60,332

JOUR At.Energ. 60, 332

JOUR At.Energ. 60, 332

JoUR At.Energ. 60,332

JoUR At.Energ. 60, 332

JoUR At.Energ. 60,332

JOUR Nucl.Instr.Meth. Phys.Res. B2B, 199

JOUR Phys.Rev. C35, 1265

JOUR Phys.Rev. C35, 1265

JOUR At.Energ. 60,332

JOUR At.Energ. 60,332

JOUR At.Energ. 60, 332

JOUR At.Energ. 60,332

JoUR At.Energ. 60,332

JoUR At.Energ. 60, 332

JOUR Yad.Fjz. 46, 28

JoUR Nucl.Jnstr.Meth. Phys.Res. B28, 199

JOUR J.Radioanal.Nucl.Chem. 121, 203

JOUR J.Radioanal.Nucl.Chem. 121, 203

JouR Nucl.1nstr.Meth. Phys.Res. B28, 199

JOUR Phys.Rev. C35, 1265

JOUR Phys.Rev. C38, 1601

JOUR J.Radioanal.Nucl.Chem. 121, 203

JoUR J.Radioanal.Nucl.Chem. 121, 203

JOUR At.Energ. 60,332

JOUR At.Energ. 60. 332

JOUR At.Enere. 60,332

JOUR At.Energ. 60, 332

JOUR At.Energ. 60,332

JoUR At.Energ. 60,332

JoUR Yad.Fiz. 46,28

JOUR J.Radioanal.Nucl.Chem, 121, 203

JOUR J.Radioanal.Nucl.Chem, 121, 203

JOUR At.Energ. 60, 332

JOUR At.Energ. 60,332

JOUR At.Energ. 60, 332

JOUR At.Energ. 60,332

JOUR At.Energ. 60,332
KEY NUMBER

$87 \operatorname{Sh} 30$

$88 \mathrm{Mu} 18$

$87 \operatorname{Sh} 30$

$87 \operatorname{Sh} 30$

87Sh 30

$86 \mathrm{Ko} 33$

$86 \mathrm{Ko3} 3$

$86 \mathrm{Ko} 33$

$86 \mathrm{Ko} 33$

$86 \mathrm{Ko} 33$

$86 \mathrm{Ko} 33$

$87 G 105$

876105

$87 \mathrm{G} 105$

$88 M{ }^{18}$

88Mu 18

$87 \mathrm{Ra} 23$

88De 9

$86 \mathrm{Ko33}$

$86 \mathrm{Ko} 33$

B6Ko33

$86 K 033$

$86 \mathrm{Ko3} 3$

$86 \mathrm{Ko} 33$

87Ra23

87 La 12

87La 12

86Ko33

$86 \mathrm{Ko} 33$

$86 \mathrm{Ko} 33$

$86 \mathrm{Ko} 33$

$86 \mathrm{Ko} 33$

$86 \mathrm{Ko} 33$

875k06

87Raz3

88 Va 14

88 Val 14

87Ra23

B7La 12

B8An1 1

BBVa 14

88Va 14

$86 \mathrm{Ko} 33$

$86 \mathrm{Ko} 33$

$86 \mathrm{Ko} 33$

$86 \mathrm{Ko} 33$

$86 \mathrm{Ko} 33$

$86 \mathrm{Ko} 33$

87Sk06

88 Va 14

B8Va 14

$86 \mathrm{Ko} 33$

$86 \mathrm{Ko} 33$

$86 \mathrm{Ko} 33$

$86 \mathrm{Ko} 33$

$86 \mathrm{Ko} 33$ 
RESIDUAL TARGET-REACTION INCIDENT ENERGY

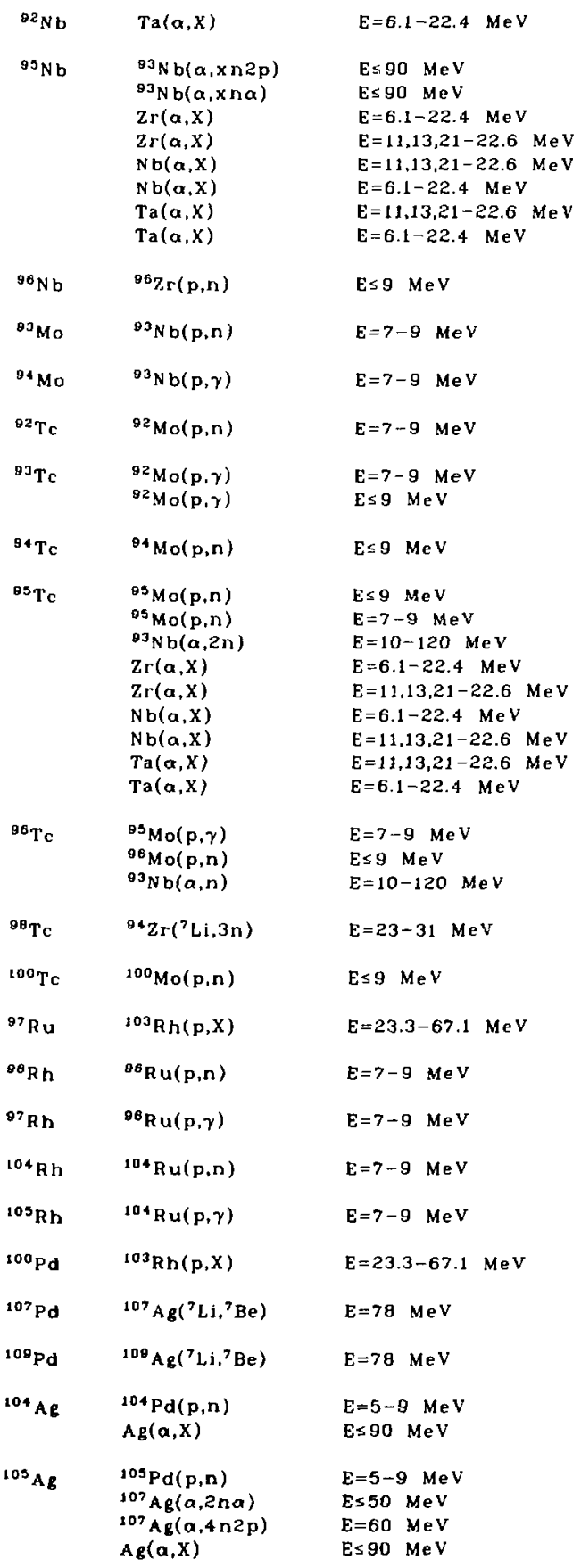

\section{REFERENCE}

JoUR At.Energ. 60,332

JoUR J.Radioanal.Nucl.Chem. 121, 203

JoUR J.Radioanal.Nucl.Chem. 121, 203

JoUR At.Energ. 60, 332

JOUR At.Energ. 60. 332

JOUR At.Energ. 60,332

JoUR At.Energ. 60, 332

JoUR At.Energ. 60,332

JoUR At.Energ. 60, 332

JOUR Yad.Fiz. 46,28

JouR Nucl.1nstr.Melh. Phys.Res. B28, 199

JouR Nucl.lnstr.Meth. Phys.Res. B28. 199

JoUR Nucl.Instr.Meth. Phys.Res. B28, 199

JouR Nucl.Instr.Meth. Phys.Res. B28, 199 JOUR Yad.Fiz. 46,28

JoliR Yad.Fiz. 46, 28

JoUR Yad.Fiz. 46, 28

JouR Nucl.Instr.Meth. Phys.Res. B2B, 199

JOUR J.Phys.(London) G13,535

JOUR At.Energ. 60,332

JoUR At.Energ. 60, 332

JoUR At.Energ. 60,332

JoUR At.Energ. 60,332

JoUR At.Energ. 60, 332

JoUR At.Energ. 60,332

JouR Nucl.Instr.Meth. Phys.Res. B28, 199 JoUR Yad.riz. 46, 28

JouR J.Phys.(London) G13, 535

JouR Phys.Rev. C36, 2330

JoUR Yad.Fiz. 46, 2B

JouR Appl.Radiat.Isot. 38, 151

JoUR Nucl.Instr.Meth. Phys.Res. B28, 199

JouR Nucl.Instr.Meth. Phys.Res. B28, 199

JouR Nucl.Instr.Meth. Phys.Res. B28, 199

JouR Nucl.Instr.Meth. Phys.Res. B28, 199

JoUR Appl.Radiat.Isot. 38, 151

KEY NUMBER

$86 \mathrm{Ko} 33$

$88 v_{a} 14$

$88 V_{a 14}$

$86 \mathrm{Ko} 33$

$86 \mathrm{Ko33}$

$86 \mathrm{~K} \circ 33$

$86 \mathrm{Ko} 33$

$86 K_{033}$

$86 \mathrm{Ko33}$

g7Sk06

87Ra23

$87 \mathrm{Ra} 23$

87Ra23

$87 \mathrm{Ra} 23$

87\$k06

87Sk06

$87 \mathrm{Sk} 06$

$87 \mathrm{Ra} 23$

$87 \mathrm{Ra} 05$

$86 \mathrm{Ko} 33$

$86 K 033$

$86 K 033$

$86 \mathrm{Ko} 33$

$86 \mathrm{Ko} 33$

$86 \mathrm{Ko} 33$

87Ra23

87Sk06

g7Ra05

87Bi21

87Sk06

87 LaOS

$87 \operatorname{Ra} 3$

$87 \mathrm{Ra} 23$

$87 \mathrm{Ra} 3$

$87 \mathrm{Ra}_{23}$

87LaO9

JOUR Yad.Fiz. 45, 1236

87G 105

JOUR Yad.Fiz. 45. 1236

$87 \mathrm{C} 105$

JOUR Yad.Fiz. 47, 609

JoUR J.Radioanal.Nucl.Chem. 121, 115

$88 \mathrm{Ba} 18$

88Wa 11

JOUR Yad.Fiz. 47, 609

JoUR Nucl.Instr.Meth. Phys.Res. B24/25, 480

JoUR Pramana 30, 193

JoUR J.Radioanal.Nucl.Chem. 121, 115
$88 \mathrm{Ba} 18$

87Si11

881501

$88 W_{\text {a }} 11$ 


\section{RESIDUAL TARGET-REACTION INCIDENT ENERGY}

\begin{tabular}{|c|c|}
\hline${ }^{106} \mathrm{Ag}$ & $\begin{array}{l}{ }^{100} \mathrm{Pd}(p, n) \\
{ }^{107} \mathrm{Ag}(\alpha, n \alpha \\
\mathrm{Ag}(\alpha, X)\end{array}$ \\
\hline${ }^{108} \mathrm{Ag}$ & $\begin{array}{l}{ }^{100} \mathrm{Pd}(\mathrm{p}, n) \\
\mathrm{Ag}(a, X)\end{array}$ \\
\hline${ }^{110} \mathrm{AB}$ & $\begin{array}{l}{ }^{110} \mathrm{Pd}(p, n) \\
\mathrm{Ag}(a, X)\end{array}$ \\
\hline${ }^{112} \mathrm{Ag}$ & $\operatorname{Ag}(a, X)$ \\
\hline${ }^{107} \mathrm{Cd}$ & ${ }^{107} \mathrm{Ag}(p, n)$ \\
\hline${ }^{108} \mathrm{Cd}$ & ${ }^{107} \mathrm{Ag}(p, \gamma)$ \\
\hline${ }^{109} \mathrm{Cd}$ & $\begin{array}{l}{ }^{109} \mathrm{Ag}(p, n) \\
{ }^{100} \mathrm{Ag}(p, n)\end{array}$ \\
\hline${ }^{110} \mathrm{Cd}$ & ${ }^{109} \mathrm{Ag}(\mathrm{p}, \gamma)$ \\
\hline${ }^{108} \ln$ & $\begin{array}{l}{ }^{107} \mathrm{Ag}(a, 2 n) \\
{ }^{109} \mathrm{Ag}(a, 4 n)\end{array}$ \\
\hline${ }^{110} 1 \mathrm{n}$ & $\begin{array}{l}110 \mathrm{Cd}(p, n) \\
{ }^{107} \mathrm{Ag}(\alpha, n) \\
{ }^{109} \mathrm{Ag}(\alpha, j 11\end{array}$ \\
\hline 111 [n & $\begin{array}{l}{ }^{11} \mathrm{Cd}(p, n) \\
111 \mathrm{Cd}(p, n) \\
{ }^{100} \mathrm{Ag}(a, 2 n) \\
{ }_{100} \mathrm{Ag}(a, 2 n)\end{array}$ \\
\hline
\end{tabular}

$1 z^{1 n} \quad{ }^{111} \mathrm{Cd}(p, \gamma)$

114 In $\quad 114 d(p, n)$

${ }^{115}$ In

$114 \mathrm{Cd}(p, \gamma)$

${ }^{114} \mathrm{Cd}(\mathbf{p}, \gamma)$

${ }^{113} \mathrm{Sn} \quad{ }_{113} \ln (p, n)$

${ }^{113} \operatorname{In}(p, n)$

${ }^{114} \mathrm{Sn} \quad{ }^{113} \ln (\mathrm{p}, \gamma)$

${ }^{115} \mathrm{Sn}$

${ }^{116} \mathrm{Sn}$

${ }^{113} \mathrm{Sb}$

${ }^{110} \mathrm{Sb}$

${ }^{117} \mathrm{Sb}$

${ }^{118} \mathrm{Sb}$

${ }^{118} \mathrm{Sb}$

${ }^{120} \mathrm{Sb}$

${ }^{122} \mathrm{Sb}$

${ }^{118} \mathrm{Te}$
$E=5-9 \mathrm{MeV}$

$\mathrm{E} \leq 50 \mathrm{MeV}$

$\mathrm{E} s 90 \mathrm{MeV}$

$E=5-9 \mathrm{MeV}$

$\mathrm{E} \leq 90 \mathrm{MeV}$

$E=5-9 \mathrm{MeV}$

Es $90 \mathrm{MeV}$

Es $90 \mathrm{MeV}$

$E=7-9 \quad \mathrm{MeV}$

$E=7-9 \mathrm{MeV}$

$E=7-9 \mathrm{MeV}$

$\mathrm{E}=9 \mathrm{MeV}$

$\mathrm{E}=7-9 \mathrm{MeV}$

$E \leq 50 \mathrm{MeV}$

$E \leq 50 \mathrm{MeV}$

$E=5.2,5.6,6 \mathrm{MeV}$

$E \leq 50 \mathrm{MeV}$

$\mathrm{E} \leq 50 \mathrm{MeV}$

$\mathrm{E}=9 \mathrm{MeV}$

$E=7-9 \quad \mathrm{MeV}$

$\mathrm{E} \leq 50 \mathrm{MeV}$

$\mathrm{E}=60 \mathrm{MeV}$

$\mathrm{E}=7-9 \mathrm{MeV}$

$E=7-9 \quad \mathrm{MeV}$

$E=7-9 \quad M e V$

$E=3-9 \mathrm{MeV}$

$E=9 \mathrm{MeV}$

$E=7-9 \quad M \in V$

$E=7-9 \mathrm{MeV}$

$E=7-9 \mathrm{MeV}$

$E=7-9 \mathrm{MeV}$

$E=3-9 \mathrm{MeV}$

$E=7-9 \mathrm{MeV}$

$E=7-9 \mathrm{MeV}$

$E=7-9 \quad M e V$

$E=7-9 \mathrm{MeV}$

$E=7-9$ MeV

$E=7-9 \quad \mathrm{MeV}$

$\mathrm{E}=9 \mathrm{MeV}$

$\mathrm{E} \approx 4-16 \mathrm{MeV}$

$E=1$ hreshold-32 MeV

\section{REFERENCE}

KEY NUMBER

JOUR Yad.Fiz. 47,609

$88 \mathrm{Ba} 18$

JOUR Nucl.Instr.Meth. Phys.Res. B24/25, 480 B7Sill

JOUR J.Radioanal.Nucl.Chem. 121, 115

88 Wa 11

JOUR Yad.Fiz. 47, 609

$88 \mathrm{Ba} 18$

JOUR J.RadjoanaJ.NucJ.Chem. I21, 115

B日Ha 11

JOUR Yad.Fiz. 47, 609

888 a18

JOUR J.Radioanal.Nucl.Chem. 121, 115

88 Wa 11

JOUR J.Radioanal.Nucl.Chem, 121, 115

88 Wall

JoUR Nucl.lnstr.Meth. Phys.Res. B28, 199

87Ra23

JOUR Nucl.Instr.Meth. Phys.Res. B28, 199

$87 R_{23}$

JoUR Nucl.Instr.Meth. Phys.Res. B28, 199 87Raz3

JOUR J.Radioanal.Nucl.Chem. $117,129 \quad$ B7Pa19

JoUR Nuclinstr.Meth. Phys.Res. 828, 199 87Ra23

JOUR Nucl.Instr.Meth. Phys.Res. 824/25, 480 87Si11

JOUR Nucl.Instr.Meth. Phys.Res. B24/25, 480 87Si 1

JOUR Nucl.Phys. A473, 471 87Kr15

JoUR Nucl.Instr.Meth. Phys.Res. B24/25, 480 87S 11

JoUR Nucl.Instr.Meth. Phys.Res. B24/25, 480 87Sil1

JOUR Yad.Fiz. 47,978 88日a 13

JoUR Nucl.Instr.Meth. Phys.Res. B28, 199 87Raz3

JoUR Nucl.Instr.Meth. Phys.Res. B24/25, 480 87Sil

JouR Pramana 30, 193 g8Is01

JOUR Nucl.Instr.Meth. Phys.Res. 828, 199 B7Raz3

JOUR Nucl.Instr.Meth. Phys.Res. B28, 199 87Raz3

JoUR Nucl.Ingtr.Meth. Phys.Res. B28, $199 \quad$ B7Raz3

JOUR Yad.Fiz. 45, 614 87 Sk03

JOUR Yad.Fiz. $47,978 \quad 88 \mathrm{Ba} 13$

JoUR Nucl.Instr.Meth. Phys.Res. B28, $199 \quad 878223$

Joyn Nucl.Instr.Meth. Phys.Res. B28, $199 \quad$ 87Raz3

JoUR Nucl.Instr.Meth. Phys.Res. B28, $199 \quad 87 R a 23$

JoUR Nucl.Instr.Meth. Phys.Res. B28, $199 \quad$ 87Raz3

JOUR Yad.Fiz. 45, 614 87Sk!3

JouR Nucl.Instr.Meth. Phys.Res. B28, $199 \quad 87$ ha23

JoUR Nucl.lnstr. Meth. Phys.Res. B28, $199 \quad 87 R a z 3$

JOUR Nucl.Instr.Meth. Phys.Res. B28, 199 B7Raz3

JouR Nucl.Instr.Melh. Phys.Res, B28, 199 B7Raz3

JOUR Nucl.Instr.Meth. Phys.Res. B28, $199 \quad 87$ Raz3

JoUR Nucl.Instr.Meth. Phys.Res. B28, $199 \quad$ 87Raz3

JOUR Yad.Fiz. $47,978 \quad 88 \mathrm{Ba} 13$

JOUR Izv.Akad.Nauk SSSR, Ser.Fiz. 52,94 88Ba52

JOUR Izv.Akad.Nauk SSSR, Ser.Fiz. 52, 94 B8Ba5? 
RESIDUAL TARGET-REACTION INCIDENT ENERGY

$\begin{array}{ll}{ }^{18} \mathrm{Te} & { }^{117} \mathrm{Sn}(a, 3 \mathrm{n}) \\ { }^{119} \mathrm{Te} & { }^{116} \mathrm{Sn}(a, \mathrm{n}) \\ & { }^{116} \mathrm{Sn}(a, n) \\ & { }^{116} \mathrm{Sn}(a, n) \\ & { }^{117} \mathrm{Sn}(a, 2 \mathrm{n}) \\ & { }^{117} \mathrm{Sn}(a, 2 \mathrm{n})\end{array}$

\begin{tabular}{|c|c|}
\hline${ }^{121} \mathrm{Te}$ & $\begin{array}{l}{ }^{121} \operatorname{Sb}(p, n) \\
{ }^{121} \operatorname{Sb}(d, 2 n) \\
121 \operatorname{Sb}(d, 2 n) \\
118 \operatorname{Sn}(\alpha, n) \\
1^{119} \operatorname{Sn}(\alpha, 2 n) \\
{ }^{120} \operatorname{Sn}(\alpha, 3 n)\end{array}$ \\
\hline${ }^{122} \mathrm{Te}$ & ${ }^{121} \mathrm{Sb}(p, y)$ \\
\hline${ }^{123} \mathrm{Te}$ & $\begin{array}{l}{ }^{123} \mathrm{Sb}(p, n) \\
127 \mathrm{~J}(p, 3 n 2 p) \\
{ }^{124} \mathrm{Sn}(\alpha, 5 n)\end{array}$ \\
\hline${ }^{12+} \mathrm{Te}$ & $\begin{array}{l}{ }^{123} \mathrm{Sb}(\mathrm{p}, \gamma) \\
{ }^{124} \mathrm{Sn}(\alpha, 4 \mathrm{n})\end{array}$ \\
\hline${ }^{125} \mathrm{Te}$ & ${ }^{124} \operatorname{Sn}(\alpha, 3 n)$ \\
\hline${ }^{126} \mathrm{Te}$ & ${ }^{124} \operatorname{sn}(\alpha, 2 n)$ \\
\hline${ }^{120} I$ & ${ }^{123} X e(p, \alpha)$ \\
\hline $121_{1}$ & ${ }^{124} \mathrm{X} e(p, \alpha)$ \\
\hline 1221 & ${ }^{126} \mathrm{Xe}(p, n \alpha)$ \\
\hline $123 l$ & $\begin{array}{l}{ }^{124} \mathrm{Xe}(p, 2 p) \\
{ }^{126} \mathrm{Xe}(p, \alpha)\end{array}$ \\
\hline 1241 & $\begin{array}{l}126 \times e(p, n 2 p) \\
{ }^{124} \mathrm{Te}(\mathrm{d}, 2 n)\end{array}$ \\
\hline${ }^{125} I_{1}$ & $\begin{array}{l}{ }^{128} \times e(p, 2 p) \\
{ }^{128} \mathrm{Xe}(p, n \alpha)\end{array}$ \\
\hline${ }^{121} \mathrm{Xe}$ & $1271(p, 7 n)$ \\
\hline${ }^{122} \mathrm{Xe}$ & ${ }^{124} \times e(p, 2 n p)$ \\
\hline${ }^{123} \mathrm{Xe}$ & $\begin{array}{l}12+\times e(p, n p) \\
124 \times e(p, n p) \\
127](p, 5 n) \\
1271(p, 5 n)\end{array}$ \\
\hline
\end{tabular}

${ }^{125} \mathrm{Xe} \quad{ }^{126} \mathrm{Xe}(\mathrm{p}, \mathrm{np})$ ${ }^{127} I(p, 3 n)$

${ }^{127} \mathrm{Xe} \quad{ }^{127} \mathrm{I}(p, n)$

${ }^{12 \theta} \mathrm{Xe} \quad{ }^{12 \gamma} \mathrm{I}(p, \gamma)$

${ }^{123} \mathrm{Cs} \quad{ }^{124} \mathrm{Xe}(p, 2 n)$ $124 \times e(p, 2 n)$

${ }^{125} \mathrm{Cs} \quad{ }^{120} \mathrm{Xe}(p, 2 n)$

${ }^{138} \mathrm{Ce} \quad{ }^{130} \mathrm{La}(p, 2 \mathrm{n})$
$E=t h r e s h o l d-32 \mathrm{MeV}$

$\mathrm{E}=20-29 \mathrm{MeV}$

$E=$ threshold $-32 \mathrm{MeV}$

$E=4-16 \mathrm{MeV}$

$E=t h r e s h o l d-32 \mathrm{MeV}$

$E=16-32 \mathrm{MeV}$

$E=7-9 \mathrm{MeV}$

$E=20-29 \mathrm{MeV}$

$E=29.3,31.7 \mathrm{MeV}$

$E=4-16 \mathrm{MeV}$

$\mathrm{E}=16-32 \mathrm{MeV}$

$E=16-32 \mathrm{MeV}$

$E=7-9 \mathrm{MeV}$

$E=7-9 \mathrm{MeV}$

$E=60 \mathrm{MeV}$

$E=35-57 \mathrm{MeV}$

$E=7-9 \quad \mathrm{MeV}$

$E=35-57 \mathrm{MeV}$

$E=35-57 \mathrm{MeV}$

$E=35-57 \mathrm{MeV}$

$E \approx 15-30 \mathrm{MeV}$

$\mathrm{E} \approx 15-30 \mathrm{MeV}$

$E \approx 15-30 \mathrm{MeV}$

$E \approx 15-30 \mathrm{MeV}$ $E \approx 15-30 \mathrm{MeV}$

$E \approx 15-30 \mathrm{MeV}$ $E=15 \mathrm{MeV}$

$\mathrm{E} \approx 15-30 \mathrm{MeV}$ $E \approx 15-30 \mathrm{MeV}$

$E=60 \mathrm{MeV}$

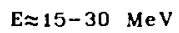

$E=15-34 \mathrm{MeV}$ $\mathrm{E} \approx 15-30 \mathrm{MeV}$

$E=60 \mathrm{MeV}$

$\mathrm{E} \approx 15-30 \mathrm{MeV}$ $E=100 \mathrm{MeV}$

$E=7-9 \mathrm{MeV}$

$E \approx 15-30 \mathrm{MeV}$

$E=15-34 \mathrm{MeV}$

$\mathrm{E} \approx 15-30 \mathrm{MeV}$

$E=16-24.5 \mathrm{MeV}$
$E=100 \mathrm{MeV}$

$E=7-9 \mathrm{MeV}$

\section{REFERENCE}

Jour Izv.Akad.Nauk SSSR, Ser.Fiz. 52, 94

JoUR I2v.Akad.Nauk SSSR, Ser.Fiz. 52, 94 JOUR Izv.Akad.Nauk SSSR, Ser.Fiz. 52, 94 JoUR lzv.Akad.Nauk SSSR, Ser.Fiz, 52, 94 JOUR lzv.Akad.Nauk SSSR, Ser.Fiz. 52, 94 JoUR Izv.Akad.Nauk SSSR, Ser.fiz. 52, 94

JoUR Nucl.Instr.Meth. Phys.Res. B28, 199 JOUR IzV.A kad.Nauk SSSR, Ser.Fiz, 52, 94 JOUR Izv.Akad.Nauk SSSR, Ser.Fiz, 52, 94 JouR Izv.Akad.Nauk SSSR, Ser.Fiz. 52, 94 JouR Izv.Akad.Nauk SSSR, Ser.Fiz, 52, 94 JOUR Izv.Akad.Nauk SSSR, Ser.Fiz. 52, 94 Jour Nucl.Instr.Meth. Phys.Res. B28, 199 JouR Nucl.Instr.Meth. Phys.Res. B28, 199 JoUR Appl.Radiat.lsot. 38,1087

JoUR Z.Phys. A 330,69

JoUR Nucl.Jnstr.Meth. Phys.Res. B28, 199 JOUR Z.Phys. A330, 69

Jouk Z.Phys, A330, 69

JouR Z.Phys. A330, 69

JOUR At.Energ. 60, 337

JoUR At.Energ. 60, 337

Jour At.Energ. 60, 337

JoUR At.Energ. 60, 337

JOUR At.Energ. 60,337

JouR At.Energ. 60, 337

JOUR J.Radioanal.Nucl.Chem. 127, 143

JOUR At.Energ. 60,337

JOUR At.Energ. 60,337

JOUR Appl.Radiat.1sot. 38, 1087

JouR At.Energ. 60, 337

JouR Radjochim. Acta 41, 1

JoUR At.Energ. 60,337

JOUR Radjokhimiya 29, 391

JOUR Appl.Radiat.Isot. 38, 1087

JoUR A t.Energ. 60, 337

JoUR Radiokhimiya 29, 391

JoUR Nucl.Instr.Meth. Phys.Res. B28, 199

JouR Nucl.instr.Meth. Phys.Res. B28, 199

JouR At.Energ. 60, 337

JoUR Radiochim.Acta 41,1

JoUR At.Energ. 60, 337

Jour Nucl.Phys. A470, 266
KEY NUMBER

88Ba52

88 8a52

88日a5?

88 Ba 52

88 Ba 52

88 Ba 52

87Ra23

$88 \mathrm{Ba} 52$

$88 B$ 5?

$88 B a 52$

B8Ba5a

888a52

$87 \mathrm{Ra} 23$

87Ra23

$87 \mathrm{Jal} 3$

88Ar09

$87 \mathrm{Raz3}$

88Ar09

88Aг09

88 A 09

86Zh08

$86 \mathrm{Zh} 08$

86Zh08

$86 \mathrm{Zh08}$

$86 \mathrm{ZhOB}$

86Zh08

88 La 15

$86 \mathrm{ZhOB}$

862h08

$87 \mathrm{Jal} 3$

862h08

B7Fi 08

86Zhos

$87 \mathrm{Za}_{1} 1$

$87 \mathrm{Ja} 13$

86Zh08

$87 \mathrm{Za}_{1} 1$

$87 \operatorname{Ra} 23$

87Ra23

B62h08

B7F j 08

B62h08

87Lo12 
RESIDUAL TARGET-REACTION INCIDENT ENERGY

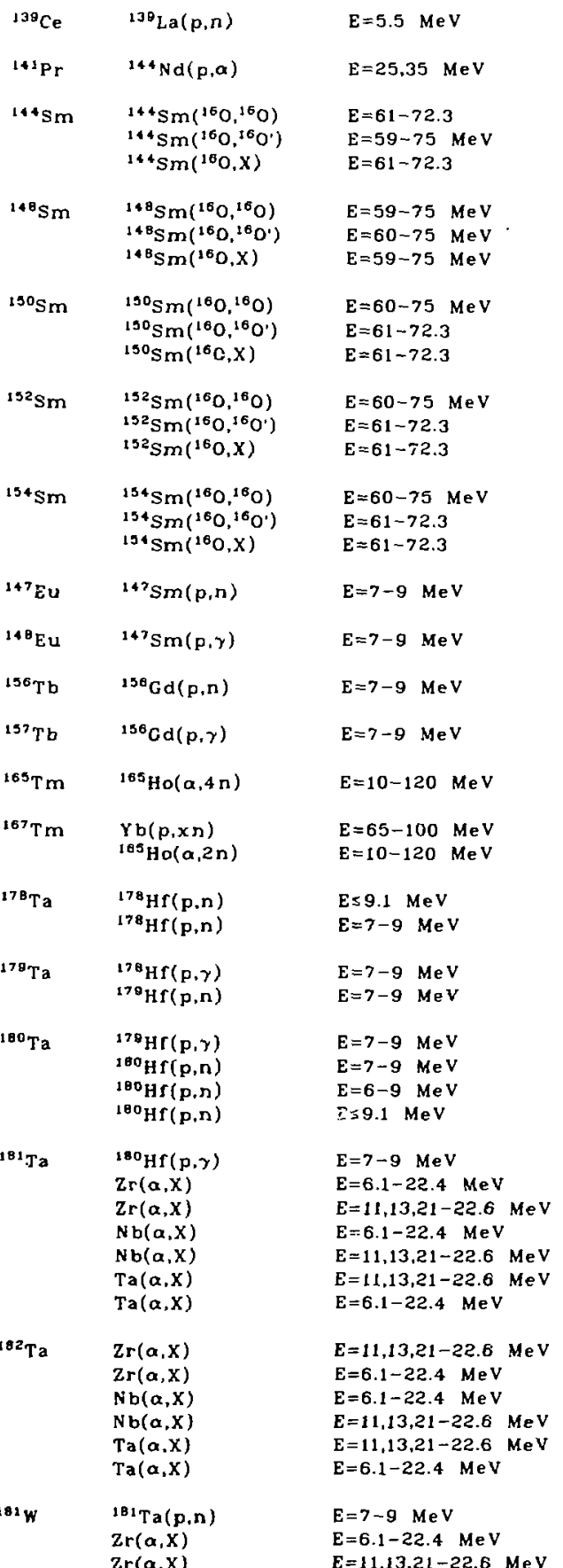

\section{REFERENCE}

JoUn Phys.Rev. C38, 625

JOUR Phys.Rev. C37, 79

JOUR Nucl.Phys. A471, 587

JOUR Nucl.Phys. A471, 587

JOUR Nucl.Phys. A471, 587

JOUR Nucl.Phys. A471, 587

JOUR Nucl.Phys. A471. 587

JOUR Nucl.Phys. A471, 587

JOUR Nucl.Phys. A471, 587

JOUR Nucl.Phys. A471, 58';

JOUR Nucl.Phys. A471, 587

JoUR Nucl.Phys. A471, 587

JoUR Nucl.Phys. A471, 587

JoUR Nucl.Phys. A471, 587

JOUR Nucl.Phys. A471, 587

JOUR Nucl.Phys. A471, 587

JOUR Nucl.Phys. A471, 587

JoUR Nucl.1nstr.Meth. Phys.Res. B28, 199

JoUR Nucl.Instr.Meth. Phys.Res. B28, 199

JoUR Nucl.1nstr.Meth. Phys.Res. B28, 199

JouR Nucl.Instr.Meth. Phys.Res. B28, 199

JOUR J.Phys.(London) G13, 535

JoUR Radiokhimiya 29, 241

JOUR J.Phys.(London) G13, 535

JOUR IzV.Akad.Nauk SSSR, Ser.Fiz. 50, 913 JoUR Nucl.Instr.Meth. Phys.Res. B28, 199

JoUR Nucl.1nstr.Meth. Phys.Res. B28, 199 JOUR Nucl.Instr.Meth. Phys.Res. B28, 199

JOUR Nucl.Instr.Meth. Phys.Res. B28, 199 JouR Nucl.Instr.Meth. Phys.Res. B28, 199 JOUR Izv.Akad.Nauk SSSR, Ser.Fiz. 50, 913 JOUR lzv.Akad.Nauk SSSR, Ser.Fiz. 50, 913

JoUR Nucl.lnstr.Meth. Phys.Res. B28, 199 JOUR At.Energ. 60, 332 JoUR At.Energ. 60,332 JOUR At.Energ. 60, 332 JOUR At.Energ. 60, 332 JoUR At.Energ. 60, 332 JoUR At.Energ. 60,332

JOUR At.Energ. 60, 332 JoUR At.Energ. 60,332 JOUR At.Energ. 60,332 JOUR At.Energ. 60,332 JoUR At.Energ. 60,332 JOUR At.Energ. 60, 332

JouR Nucl.Instr.Meth. Phys.Res. B28, 199 JoUR At.Energ. 60,332 JOUR At.EnErg. 60,332
KEY NUMBER

$88 \mathrm{Ch} 23$

88Ga 01

$87 K+19$

$87 K 119$

$87 K+19$

$87 \mathrm{~K}$ i 19

$87 K+19$

$87 \mathrm{~K} 119$

$87 K 119$

$87 K$ i 19

87Ki 19

$87 K+19$

$87 K, 19$

87K I 19

$87 \mathrm{~K} 19$

$87 \mathrm{~K} 19$

87K 19

87Ra23

87Ra23

87Ra23

87Ra23

87Ra05

87NR05

$87 \mathrm{KaO} 05$

$86 \mathrm{Ba} 84$

$87 \mathrm{Ra}_{23}$

87Ra23

87Ra23

87Ra23

$87 \mathrm{Ra} 23$

$86 \mathrm{Ba} 84$

$868 \mathrm{a} 84$

$87 \mathrm{Ra}_{3}$

$86 K 033$

$86 \mathrm{Ko} 33$

$86 K 033$

$86 K 033$

$86 \mathrm{Ko} 33$

$86 \mathrm{Ko} 33$

$86 \mathrm{Ko} 33$

$86 \mathrm{Ko} 33$

$86 K 033$

$86 K 033$

$86 K 033$

$86 K 033$

87Ra 23

$86 K 033$

$86 \mathrm{Ko33}$ 


\begin{tabular}{|c|c|c|c|c|c|}
\hline RESIDUAL & TARGET-REACT ION & 1NCIDENT ENERGY & REFE & ERENCE & KEY NUMBER \\
\hline \multirow[t]{4}{*}{${ }^{101} \mathrm{~W}$} & $\mathrm{Nb}(\alpha, \mathrm{X})$ & $\mathrm{E}=11,13,21-22.6 \mathrm{MeV}$ & JOUR & A t.Energ. 60,332 & $86 K \circ 33$ \\
\hline & $\mathrm{Nb}(\alpha, X)$ & $E=6.1-22.4 \mathrm{MeV}$ & JOUR & At.Energ. 60,332 & $86 K \circ 33$ \\
\hline & $\mathrm{Ta}(a, X)$ & $\mathrm{E}=6.1-22.4 \mathrm{MeV}$ & JOUR & At.Energ. 60,332 & $86 \mathrm{Ko3} 3$ \\
\hline & $\mathrm{Ta}(a, X)$ & $E=11.13,21-22.6 \mathrm{MeV}$ & JOUR & At.Energ. 60,332 & $86 \mathrm{~K} 033$ \\
\hline $182 w$ & ${ }^{181} \mathrm{Ta}(p, \gamma)$ & $\mathrm{E}=7-9 \mathrm{MeV}$ & JOUR & Nucl.lnstr.Meth. Phys.Res. B28, 199 & $87 \mathrm{Ra23}$ \\
\hline${ }^{181} \mathrm{Re}$ & ${ }^{181} \mathrm{Ta}(a, 4 n)$ & $\mathrm{E}=60 \mathrm{MeV}$ & JOUR & Pramana 30,193 & 881501 \\
\hline${ }^{182} \mathrm{Re}$ & ${ }^{181} \mathrm{Ta}(\alpha, 3 \mathrm{n})$ & $\mathrm{E}=60 \mathrm{MeV}$ & JOUR & Pramana 30,193 & $881 \mathrm{~s} 01$ \\
\hline \multirow[t]{8}{*}{${ }^{\llcorner 83} \mathrm{Re}$} & ${ }^{183} W(p, n)$ & $E=7-9 \mathrm{MeV}$ & JOUR & Nucl.Instr.Meth. Phys.Res, B28, 199 & $87 \mathrm{Ra} 23$ \\
\hline & ${ }^{181} \mathrm{Ta}(\alpha, 2 \mathrm{n})$ & $\mathrm{E}=60 \mathrm{MeV}$ & JOUR & Pramana 30,193 & $881 \leq 01$ \\
\hline & $\operatorname{Zr}(a, x)$ & $E=11,13,21-22.6 \mathrm{MeV}$ & JOUR & At.Energ. 60,332 & $86 \mathrm{~K} 033$ \\
\hline & $\operatorname{Zr}(a, x)$ & $E=6.1-22.4 \mathrm{MeV}$ & JOUR & At.Energ. 60,332 & $86 \mathrm{Ko} 33$ \\
\hline & $\mathrm{Nb}(a, X)$ & $E=11,13,21-22.6 \mathrm{MeV}$ & JOUR & At.Energ. 60,332 & $86 \mathrm{Ko33}$ \\
\hline & $\operatorname{Nb}(a, x)$ & $E=6.1-22.4 \quad \mathrm{MeV}$ & JOUR & At.Energ. 60, 332 & $86 K 033$ \\
\hline & $\operatorname{Ta}(a, X)$ & $\mathrm{E}=6.1-22.4 \mathrm{MeV}$ & JOUR & At.Energ. 60,332 & $86 K 033$ \\
\hline & $\operatorname{Ta}(a, x)$ & $E=11,13,21-22.6 \mathrm{MeV}$ & JouR & At.Energ. 60,332 & $86 \mathrm{Ko3} 3$ \\
\hline \multirow[t]{8}{*}{${ }^{164} \mathrm{Re}$} & $183 w(p, \gamma)$ & $E=7-9 \mathrm{MeV}$ & JOUR & Nucl.Instr.Meth. Phys.Res. B28, 199 & $87 \mathrm{Ra23}$ \\
\hline & ${ }^{181} \mathrm{Ta}(a, n)$ & $E=60 \mathrm{MeV}$ & JOUR & Pramana 30,193 & $881 \mathrm{s01}$ \\
\hline & $\operatorname{Zr}(a, X)$ & $\mathrm{E}=11,13,21-22.6 \mathrm{MeV}$ & JOUR & At.Energ. 60, 332 & $86 K \circ 33$ \\
\hline & $\operatorname{Zr}(\alpha, X)$ & $E=6.1-22.4 \mathrm{MeV}$ & JOUR & At.Energ. 60,332 & $86 K o 33$ \\
\hline & $\mathrm{Nb}(a, \mathrm{X})$ & $\mathrm{E}=6.1-22.4 \mathrm{MeV}$ & JOUR & At.Energ. 60,332 & $86 \mathrm{Ko} 33$ \\
\hline & $\mathrm{Nb}(a, \mathrm{X})$ & $E=11,13,21-22.6 \mathrm{MeV}$ & JOUR & At.Energ. 60,332 & $86 \mathrm{Ko33}$ \\
\hline & $\mathrm{Ta}(a, X)$ & $E=6.1-22.4 \mathrm{MeV}$ & JOUR & At.Energ. 60,332 & $86 K 033$ \\
\hline & $\operatorname{Ta}(\alpha, x)$ & $E=11,13,21-22.6 \mathrm{MeV}$ & JOUR & At.Energ. 60,332 & $\mathrm{~B} 6 \mathrm{Ko33}$ \\
\hline${ }^{197} \mathrm{Pt}$ & ${ }^{107} \mathrm{Au}\left({ }^{7} \mathrm{Li},{ }^{7} \mathrm{Be}\right)$ & $E=7 B \quad \mathrm{MeV}$ & JouR & Yad.Fiz. 45,1236 & $87 G 105$ \\
\hline \multirow[t]{2}{*}{${ }^{104} \mathrm{~A} u$} & ${ }^{104} \mathrm{Pt}(\mathrm{p}, \mathrm{n})$ & $E=7-9 \mathrm{MeV}$ & JOUR & Nucl.Instr,Meth. Phys.Res. B28, 199 & $87 \mathrm{Ra} 23$ \\
\hline & ${ }^{197} \mathrm{~A} \cup(a, 3 \mathrm{n} a)$ & $E=t h$ reshold $-75 \mathrm{MeV}$ & JOUR & J.Phys.(London) G14, 931 & B8si 10 \\
\hline \multirow[t]{2}{*}{$195 \mathrm{~A} \mathrm{u}$} & ${ }^{194} \mathrm{Pt}(\mathrm{p}, \gamma)$ & $\mathrm{E}=7-9 \mathrm{MeV}$ & JOUR & Nucl.Instr.Meth. Phys.Res. B2B, 199 & 87Ra23 \\
\hline & ${ }^{195} \mathrm{Pt}(\mathrm{p}, \mathrm{n})$ & $\mathrm{E}=7-9 \mathrm{MeV}$ & JoUR & Nucl.Instr.Meth. Phys.Res. B28, 199 & $87 \mathrm{Ra} 23$ \\
\hline \multirow[t]{3}{*}{${ }^{190} \mathrm{Au}$} & ${ }^{195} \mathrm{Pt}(\mathrm{p}, \gamma)$ & $E=7-9 \mathrm{MeV}$ & JOUR & Nucl.lnstr.Meth. Phys.Res. B28, 199 & $87 R a 23$ \\
\hline & ${ }^{197} \mathrm{~A} u(d, 2 \Omega p)$ & $E=6.6-13.1 \mathrm{MeV}$ & JOUR & Chin.J.Nucl.Phys. 9, 48 & $87 \mathrm{~L} 007$ \\
\hline & ${ }^{107} \mathrm{~A} \cup(\alpha, n \alpha)$ & $\mathrm{E}=$ threshold $-75 \mathrm{MeV}$ & JOUR & J.Phys.(London) G14, 931 & 885 i 10 \\
\hline \multirow[t]{2}{*}{${ }^{198} \mathrm{Au}$} & ${ }^{197} \mathrm{~A} \cup(\mathrm{d}, \mathrm{p})$ & $\mathrm{E}=6.6-13.1 \mathrm{MeV}$ & JOUR & Chin.J.Nucl.Phys. 9, 48 & $87 \mathrm{Lo0} 07$ \\
\hline & ${ }^{187} \mathrm{~A} u(\alpha, n 2 p)$ & $E=t h r e s h o l d-75 \mathrm{MeV}$ & JOUR & J.Phys.(London) G14, 931 & $88 S$ i 10 \\
\hline \multirow[t]{2}{*}{${ }^{187} \mathrm{Hg}$} & ${ }^{197} \mathrm{~A} u(p, n)$ & $\mathrm{E}=7-9 \mathrm{MeV}$ & JOUR & Nucl.Instr.Meth. Phys.Res. 828, 199 & $87 \operatorname{Ra} 23$ \\
\hline & ${ }^{197} \mathrm{~A} \cup(\mathrm{d}, 2 \pi)$ & $E=6.6-13.1 \mathrm{MeV}$ & JOUR & Chin.J.Nucl.Phys. 9,48 & B7L007 \\
\hline${ }^{188} \mathrm{Hg}$ & ${ }^{107} \mathrm{~A} u(p, \gamma)$ & $E=7-9 \mathrm{MeV}$ & JOUR & Nucl.Irstr.Meth. Phys.Res, B2B, 199 & B7Ra23 \\
\hline $108 \mathrm{Tl}$ & ${ }^{197} \mathrm{~A} \cup(\alpha, 3 n)$ & $E=t h r e s h o l d-75 \mathrm{MeV}$ & JOUR & J.Phys.(London) G14,931 & $88 \mathrm{Si} 10$ \\
\hline$\left.{ }^{189} \mathrm{~T}\right]$ & ${ }^{197} \mathrm{~A} u(a, 2 n)$ & $E=t h$ reshold $-75 \mathrm{MeV}$ & JOUR & J.Phys.(London) G14, 931 & $885 i 10$ \\
\hline${ }^{200} \mathrm{Tl}$ & ${ }^{197} \mathrm{~A} u(a, n)$ & $E=t h r e s h o l d-75 \mathrm{MeV}$ & Jour & J.Phys.(London) G14, 931 & $885 i 10$ \\
\hline${ }^{201} \mathrm{Tl}$ & $\mathbf{P b}(p, x n)$ & $\mathrm{E}=65 \mathrm{MeV}$ & JOUR & Radiokhimiya 29,247 & $872 a 09$ \\
\hline${ }^{202} \mathrm{Tl}$ & $P b(p, x n)$ & $E=65 \mathrm{MeV}$ & JOUR & Radiokhimiya 29,247 & $872 \mathrm{a09}$ \\
\hline${ }^{208} \mathrm{Tl}$ & ${ }^{208} \mathrm{~Pb}\left({ }^{7} \mathrm{Li},{ }^{7} \mathrm{Be}\right)$ & $E=7 B \quad \mathrm{MeV}$ & JOUR & Yad.Fiz. 45,1236 & $87 G 105$ \\
\hline \multirow[t]{2}{*}{${ }^{200} \mathrm{~Pb}$} & ${ }^{200} \mathrm{~Pb}(\mathrm{~d}, \mathrm{np})$ & $\mathrm{E} \approx 10-250 \mathrm{MeV}$ & JOUR & Nucl.Phys. A 485, 221 & 8gSro2 \\
\hline & ${ }^{200} \mathrm{~Pb}\left({ }^{3} \mathrm{He}, \mathrm{pd}\right)$ & $\mathrm{E} \approx 10-250 \mathrm{MeV}$ & JOUR & Nucl.Phys. A485, 221 & $88 \mathrm{Sr} 02$ \\
\hline${ }^{205} \mathrm{Bi}$ & $P b(p, X)$ & $\mathrm{E}=8.8-67.5 \mathrm{MeV}$ & JOUR & Appl.Radiat.Isot. 38, 129 & e7 LaOB \\
\hline
\end{tabular}




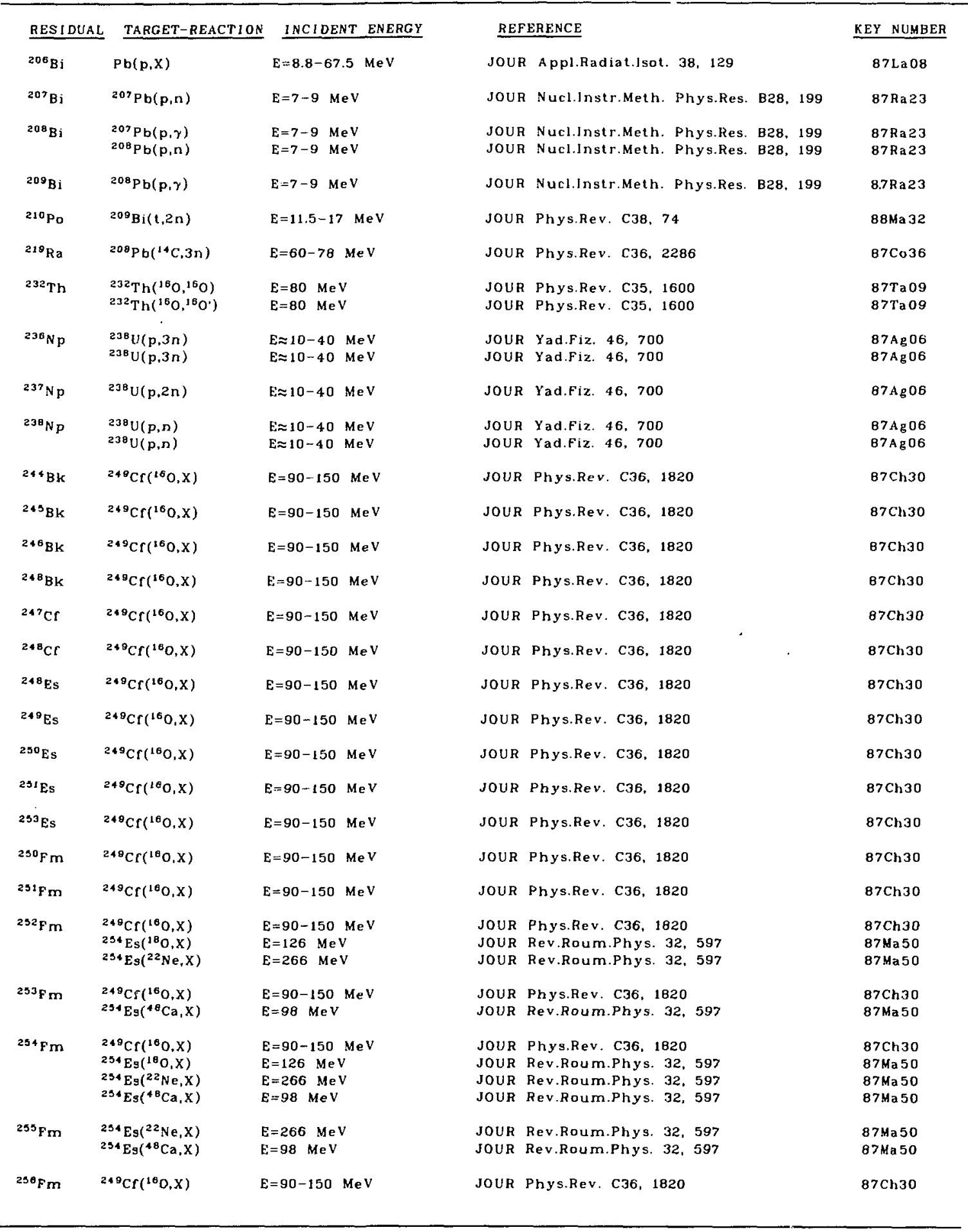




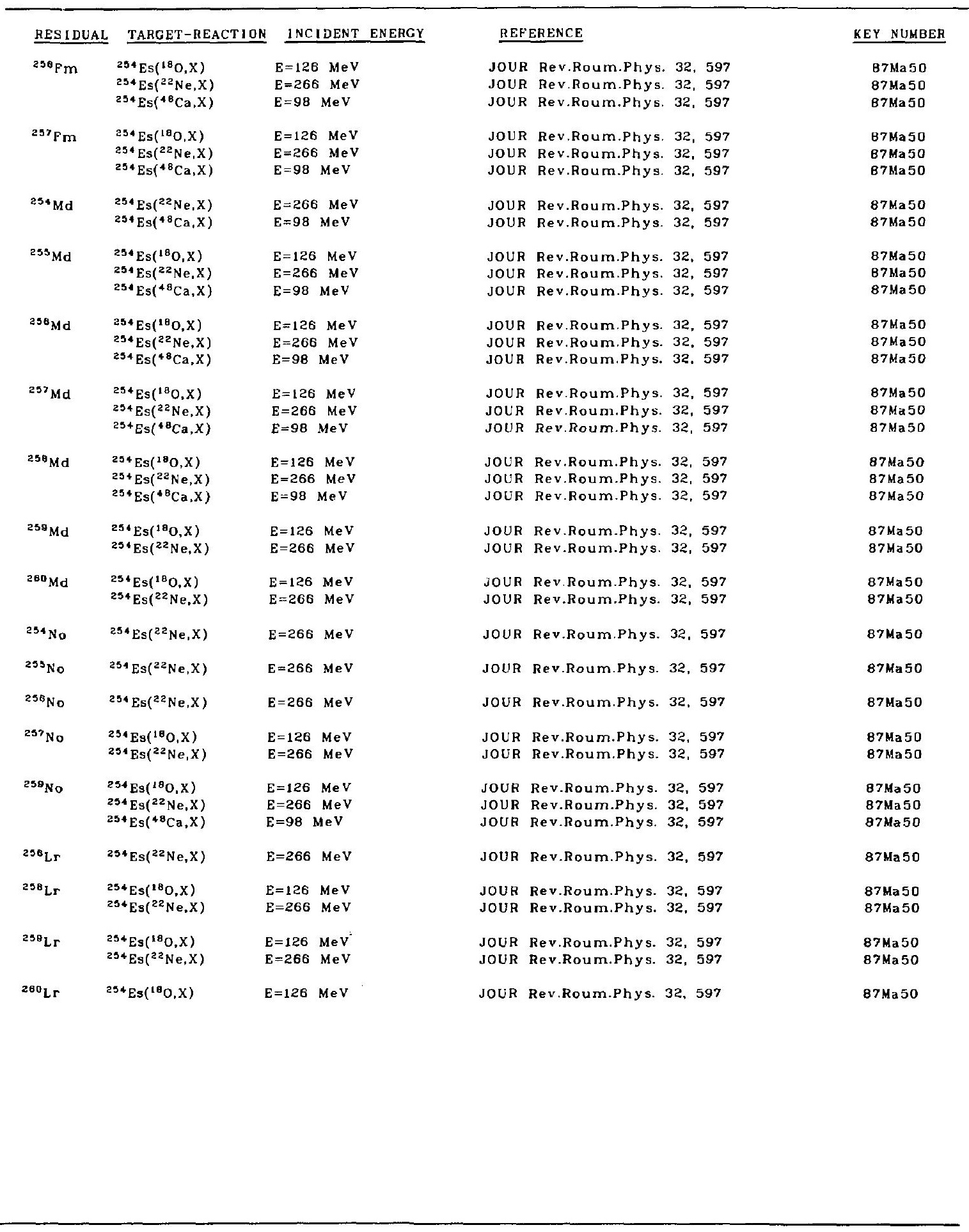

